\begin{abstract}
Title of dissertation: $\quad$ CFD/CSD STUDY OF INTERACTIONAL AERODYNAMICS OF A COAXIAL COMPOUND HELICOPTER IN HIGH-SPEED FORWARD FLIGHT

Vera Klimchenko, Doctor of Philosophy, 2020

Dissertation directed by: Professor James Baeder Department of Aerospace Engineering
\end{abstract}

This work presents a computational study of the aerodynamic interactions that arise between the components of a high-speed lift-offset coaxial compound helicopter in forward flight. The objective of this study is to develop a computational methodology that would enable fundamental understanding of the complex aeromechanics of a modern lift-offset coaxial compound rotorcraft configuration in it's entirety. The modeling of a helicopter is a coupled aeroelastic problem, in which the aerodynamics is highly dependent on the structural dynamics, and vice versa. Therefore, the prediction of the rotorcraft airloads and blade deformations must be performed with sufficient fidelity to accurately model both aspects of the problem.

A high-fidelity computational fluid dynamics framework, HPCMP CREATE ${ }^{T M_{-}}$ AV Helios, was used in conjunction with an in-house comprehensive analysis solver, to simulate a lift-offset coaxial compound helicopter in forward flight. A notional X2TD helicopter consisting of a lift-offset coaxial rotor, airframe and an aft-mounted propeller, was modeled in this work. An in-house comprehensive analysis solver, PRASADUM, performed trim calculations and the structural modeling using low 
order aerodynamics.

Conventionally, the comprehensive analysis rotor airloads that are computed from the built-in low order aerodynamic models, would be corrected with the highfidelity CFD airloads using delta coupling procedure. In this study, the conventional rotor delta coupling methodology was used to study the interactional aerodynamics of a coaxial rotor system in forward flight at a range of flight speeds (50 knots to 225 knots). This study also focused on extending this methodology to perform high-fidelity airloads corrections for airframe and the propeller. The low order rotor, airframe and propeller aerodynamic loads were corrected with the high-fidelity CFD airloads, using a full vehicle loose delta coupling methodology. The two CFD/CSD coupling approaches, rotor and full vehicle, were compared. The results showed that correcting the low fidelity CSD airframe airloads with high-fidelity CFD airloads affects the rotor trim solution.

The converged trim state from the full vehicle delta coupling procedure was utilized to study the fundamental interactional aerodynamics between various components of the coaxial compound helicopter. The CFD simulations were performed for isolated helicopter components and component combinations. 


\title{
CFD/CSD STUDY OF INTERACTIONAL AERODYNAMICS OF A COAXIAL COMPOUND HELICOPTER IN HIGH-SPEED FORWARD FLIGHT
}

\author{
by \\ Vera Klimchenko \\ Dissertation submitted to the Faculty of the Graduate School of the \\ University of Maryland, College Park in partial fulfillment \\ of the requirements for the degree of \\ Doctor of Philosophy \\ 2020
}

\author{
Advisory Committee: \\ Professor James Baeder, Chair/Advisor \\ Professor Anubhav Datta \\ Professor Inderjit Chopra \\ Professor Alison Flatau \\ Professor Kenneth Kiger
}


(c) Copyright by

Vera Klimchenko

2020 
For Mom, Dad and Mike. 


\section{Acknowledgments}

Foremost, I would like to express my deep appreciation and gratitude to my family and my dearest friends. Thank you for supporting me on this wonderful journey. Your overwhelming support and love sustained me throughout the years. I am sincerely thankful for each and every one of you.

I am grateful to my Ph.D. advisor, Dr.James Baeder, for graciously granting me the opportunity to perform research in his group and introducing me to the complex world of computational fluid dynamics.

I would like to extend a heartfelt thank you to Dr.Anubhav Datta for his guidance, motivation and support during the final stages of my graduate studies. His constructive criticism and mentoring have been invaluable. He never ceased to believe in me and my abilities, I will be forever grateful to him.

I would like to thank Dr.Alison Flatau for allowing me the opportunity to conduct undergraduate research in her laboratory. It was a gateway to the world of research and graduate studies. I would like to show my deep appreciation to Dr.Anya Jones, my M.S. advisor, for teaching me the essential skills that I would need to succeed as a graduate student. Also, I would like to thank Dr.Inderjit Chopra for encouraging me to take the Helicopter Design course. This course was truly a rewarding experience which changed my academic and research perspectives.

Finally, I would like to give my appreciation to the VLRCOE technical mentor, Dr.Mahendra Bhagwat, and the VLRCOE committee for supporting my Ph.D. project and providing valuable constructive criticism. 


\section{Table of Contents}

List of Tables vii

List of Figures viii

Nomenclature xii

1 Introduction 1

1.1 Background and Motivation . . . . . . . . . . . . . . . . . . . 1

1.2 Summary of Previous Work . . . . . . . . . . . . . . . . . . . . . . . 9

1.2.1 Experimental Studies . . . . . . . . . . . . . . . . . . 10

1.2 .2 CFD/CSD Simulations - SMR Configurations . . . . . . . . . 13

1.2.3 CFD/CSD Simulations - Coaxial Rotor Configurations . . . . 16

1.3 Contributions of Present Research . . . . . . . . . . . . . . . . 20

1.4 Scope and Organization of Thesis . . . . . . . . . . . . . . . . . . . . 22

2 Notional High-Speed Coaxial Compound Helicopter 24

2.1 Coaxial Rotor . . . . . . . . . . . . . . . . . . . . 24

2.2 Auxiliary Propulsor . . . . . . . . . . . . . . . . . . . . . . . . . . . . 32

2.3 Airframe . . . . . . . . . . . . . . . . . . . . . . . . 34

3 Comprehensive Analysis 37

3.1 Rigid Body Dynamics . . . . . . . . . . . . . . . . . . . . . 37

3.2 Aerodynamic Model . . . . . . . . . . . . . . . . . . . . . . . . . 40

3.2 .1 Fuselage Airloads . . . . . . . . . . . . . . . . . . . . . . . . . 40

3.2.2 $\quad$ Empennage Airloads . . . . . . . . . . . . . . . . . . . . . . . 41

3.2.3 Auxiliary Propeller Airloads . . . . . . . . . . . . . . . . . . . 42

3.2 .4 Coaxial Rotor System Airloads . . . . . . . . . . . . . . . . . 43

3.3 Structural Model . . . . . . . . . . . . . . . . . . . . 46

3.4 Rotor Controls . . . . . . . . . . . . . . . . . . . . . . . . . . . . . . 48

3.5 Trim . . . . . . . . . . . . . . . . . . . . . 50

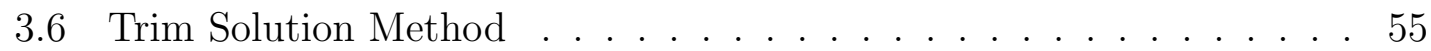

3.7 Validation of the Notional X2TD Comprehensive Analysis Model . . 56 
\begin{tabular}{|lll}
4 & Computational Fluid Dynamics & 59
\end{tabular}

4.1 Governing Equations . . . . . . . . . . . . . . . . . . . . . . . 59

4.2 Reynolds Averaged Navier-Stokes Equations . . . . . . . . . . . . . 62

$4.3 \quad$ CFD Solvers . . . . . . . . . . . . . . . . . . . . . . . . . . 63

4.3 .1 Coaxial Main Rotor - OVERFLOW . . . . . . . . . . . . . 65

4.3 .2 Airframe and Propeller - FUN3D . . . . . . . . . . . . 66

$4.3 .3 \quad$ Off-body - SAMCart . . . . . . . . . . . . . . . . . . . . . . . 69

4.4 Blade Motion and Deformation . . . . . . . . . . . . . . . 70

\begin{tabular}{|lll}
5 & CFD/CSD Coupling Methodology & 72
\end{tabular}

$5.1 \quad$ Rotor CFD/CSD Coupling . . . . . . . . . . . . . . . . . . . 72

5.2 Full Vehicle CFD/CSD Coupling _ . . . . . . . . . . . . . . 74

$5.3 \quad$ CFD/CSD Coupling Validation $\ldots \ldots \ldots \ldots . \ldots . \ldots 77$

6 Results Part I: Coaxial Rotor CFD/CSD Coupling 79

6.1 Main Rotor Controls and Vehicle Pitch Attitude . . . . . . . . . . . . 81

6.1 .1 Rotor Collective $\left(\theta_{0}\right)$ and Fuselage Pitch . . . . . . . . . 81

6.1.2 Cyclic Pitch $\theta_{1 s}$ and Cyclic Pitch $\theta_{1 c} \quad \ldots \ldots$. . . . . 84

6.2 Blade Pitch and Flap . . . . . . . . . . . . . . . . . . . . . . 84

6.3 Rotor Airloads . . . . . . . . . . . . . . . . . . . . . . . . . 87

6.4 Coaxial Rotor Interactional Aerodynamics - 50 Knots . . . . . . . . . 93

7 Results Part II: Vehicle CFD/CSD Coupling 104

7.1 Trim Parameters . . . . . . . . . . . . . . . . . . . . . . 105

7.2 Main Rotor Blade Pitch and Flap . . . . . . . . . . . . . . . . . . 111

7.3 Main Rotor Airloads . . . . . . . . . . . . . . . . . . . . . . 115

7.3.0.1 Normal Force - Upper and Lower Rotor . . . . . . . 116

7.3.0.2 Chordwise Force - Upper and Lower Rotor . . . . . . 122

7.3.0.3 Pitching Moment - Upper and Lower Rotor . . . . . 124

7.4 Structural Blade Loads . . . . . . . . . . . . . . . . . . . . 130

7.5 Pusher Propeller $\ldots \ldots \ldots$. . . . . . . . . . . . . . . . 134

8 Results Part III: Fundamental Understanding of Interactional Aerodynamics of Compound Coaxial Helicopter 136

8.1 Interactional Aerodynamics Affecting the Upper Rotor . . . . . . . . 138

8.2 Interactional Aerodynamics Affecting the Lower Rotor . . . . . . . . 143

8.3 Interactional Aerodynamics Affecting the Airframe . . . . . . . . . 148

8.4 Interactional Aerodynamics Affecting the Auxiliary Propeller . . . . . 159

9 Conclusions 162

9.1 Summary of Research . . . . . . . . . . . . . . . . . . . . . . 162

9.2 Key Conclusions $\ldots \ldots \ldots$. . . . . . . . . . . . . . . . 163

9.2 .1 Coaxial Rotor CFD/CSD Coupling . . . . . . . . . . . 164

9.2 .2 Results Part II: Vehicle CFD/CSD Coupling . . . . . . . . . 165 
9.2.3 Results Part III: Fundamental Understanding of Interactional Aerodynamics of Compound Coaxial Helicopter . . . . . . . . 167

9.3 Recommendations for Future Work . . . . . . . . . . . . . . . . . . . 168

\begin{tabular}{ll}
\hline B Coordinate Systems & 170
\end{tabular}

B Flow Visualization for Results Part I 172

C Flow Visualization for Results Part III 174

D Collective Sweep for Isolated Auxiliary Propeller and Airframe$\begin{array}{ll}\text { Propeller Combination } & 176\end{array}$

\begin{tabular}{ll}
\hline Bibliography & 177
\end{tabular} 


\section{List of Tables}

2.1 The X2TD rotor system parameters . . . . . . . . . . . . . . . . . 25

2.2 The notional X2TD auxiliary propeller parameters $[1]$. . . . . . . . 33

4.1 Grid spacing per level in terms of root chords for the off-body Cartesian mesh. . . . . . . . . . . . . . . . . 70

6.1 Coaxial CFD/CSD coupling forward flight speeds. . . . . . . . . . . . 80

6.2 The final coaxial CFD/CSD controls and vehicle pitch attitude at various speeds. The control angles and the vehicle pitch attitude are shown in degrees. . . . . . . . . . . . . . . . . . . . . 82

6.3 The final coaxial CFD/CSD controls and vehicle pitch attitude at 50 knots with interactional effects (Case 1) and without interactional effects (Case 2). . . . . . . . . . . . . . . . . 97

$7.1 \quad$ CFD interactional aerodynamics case . . . . . . . . . . . . . . 105

7.2 Comparison between final coaxial CFD/CSD and full vehicle CFD/CSD coupling at 150 knots. . . . . . . . . . . . . . . . . . . . 107

7.3 Comparison between final coaxial CFD/CSD and full vehicle CFD/CSD pitch deflection coupling at 150 knots. . . . . . . . . . . . . . . 112

8.1 CFD interactional aerodynamics case . . . . . . . . . . . . . . . . . . 138

8.2 Mean auxiliary propeller thrust and power for three configurations at 150 knots. . . . . . . . . . . . . . . . . 160 


\section{List of Figures}

1.1 A high-speed coaxial compound helicopter, Sikorsky-Boeing SB>1

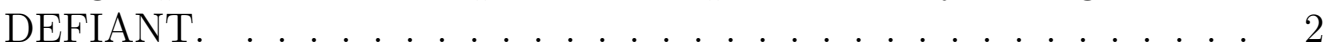

1.2 A conventional single main rotor helicopter, Sikorsky UH-60 Black Hawk. . . . . . . . . . . . . . . . . . 2

1.3 The limitations on a helicopter rotor which effect forward flight speed. 4

1.4 An example of edgewise velocity distribution around the rotor disk. . 4

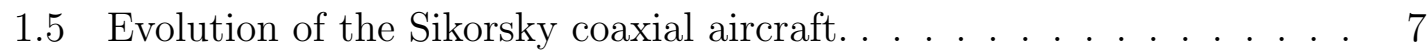

1.6 Experimental testing of coaxial rotor configurations. . . . . . . . . . 11

$1.7 \quad$ Hart II CFD/CSD study presented in Ref. [2] . . . . . . . . . . . . 15

1.8 The iso-surfaces of vorticity from the CFD and VVPM simulations presented by Jacobellis et al. in Ref. $|3| . \ldots \ldots$. . . . . . . . . 18

2.1 A flight test of the Sikorsky X2 Technology Demonstrator Ref. [4].] . . 25

2.2 Sikorsky X2TD and XH59-A main rotor blade thickness distribution |5|. 27

2.3 Notional X2TD rotor blade. $\ldots \ldots \ldots \ldots \ldots \ldots \ldots$

2.4 Notional X2 rotor blade parameters. . . . . . . . . . . . . . . . . . . 29

2.5 Main rotor RPM ratio for X2TD obtained from Ref. $[5]$ and lateral LOS obtained from Ref. $|6| . \ldots \ldots \ldots \ldots . \ldots \ldots 31$

2.6 Auxiliary propeller power required at $4 \mathrm{~K}$ density altitude, obtained

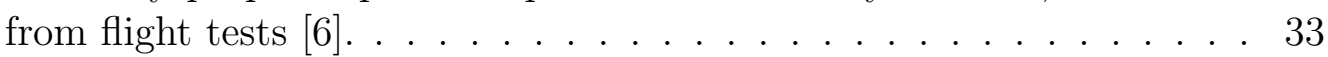

2.7 Notional X2TD auxiliary propeller twist and chord distribution. . . . 34

2.8 Notional X2TD airframe. . . . . . . . . . . . . . . . 36

2.9 Sikorsky X2TD during it's first flight on August 27, 2008 |6|. . . . . . 36

3.1 The power validation for the notional X2TD. . . . . . . . . . . . . 57

3.2 The fanplot validation for the notional X2TD. . . . . . . . . . . 58

4.1 Helicopter mesh system information. . . . . . . . . . . . . . . . 64

4.2 Main rotor blade mesh. . . . . . . . . . . . . . . . . . . 66

4.3 Airframe near-body surface mesh. . . . . . . . . . . . . . . . . . 68

4.4 Notional X2TD auxiliary propeller near-body surface mesh. . . . . . . 68

4.5 Mesh domain viewed from port side of the helicopter. . . . . . . . . . 69

5.1 Conventional, rotor CFD/CSD coupling schematic. . . . . . . . . 73 
$5.2 \quad$ Full vehicle CFD/CSD coupling schematic. . . . . . . . . . . . . . . . 75

5.3 Comparison of predicted CFD/CSD airloads with the wind tunnel test data for UH-60A rotor. . . . . . . . . . . . . . . . . . . . . . 78

6.1 The coaxial CFD/CSD coupling mesh system and Q-criterion flow visualization for coaxial rotor at 50 knots. . . . . . . . . . . . . . . . 80

6.2 The final coaxial CFD/CSD main rotor controls (a) and vehicle pitch attitude (b) at various speeds. . . . . . . . . . . . . . . 82

6.3 Upper rotor and lower rotor tip pitch deflections for coaxial and full vehicle CFD/CSD coupling at a range of forward flight speeds. . . . . 86

6.4 Upper rotor and lower rotor tip flap deflections for coaxial and full vehicle CFD/CSD coupling at a range of forward flight speeds. . . . . 86

6.5 The upper rotor airloads for a range of speeds. . . . . . . . . . . . 88

6.6 The lower rotor airloads for a range of speeds. . . . . . . . . . . . . . . . . 89

6.7 The upper(a) and lower(b) rotor sectional normal force for a range of

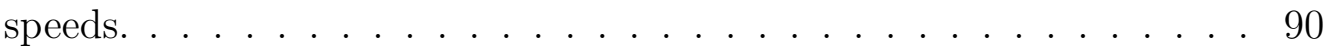

6.8 The upper (a) and lower rotor (b) sectional pitching moment for a range of speeds. . . . . . . . . . . . . . . . . . . 91

6.9 The upper and lower rotor normal force at 50 knots for interactional and non-interactional case utilizing deflections from CSD0. . . . . . . 95

6.10 The upper and the lower rotor tip pitch and flap deflections for interactional and non-interacting cases at 50 knots. . . . . . . . . . . 97

6.11 The upper (a) and lower (b) rotor normal force at 50 knots for interactional and non-interactional case at final CFD/CSD coupling iteration. . . . . . . . . . . . . . . . . . . 100

6.12 The upper (a) and lower (b) rotor sectional normal force at 50 knots for interactional and non-interactional case. . . . . . . . . . . . . . . 101

6.13 The upper and lower rotor pitching moment at 50 knots for interactional and non-interactional case. . . . . . . . . . . . . . . . . . . 102

6.14 The upper (a) and lower (b) rotor sectional normal force at 50 knots for interactional and non-interactional case. . . . . . . . . . . . . . . 103

7.1 Rotor controls and fuselage attitude convergence history for coax and full vehicle CFD/CSD coupling at 150 knots. . . . . . . . . . . . . . . 108

7.2 Convergence of airframe loads at CG at 150 knots. . . . . . . . . . . 109

7.3 Pusher propeller thrust convergence at 150 knots. . . . . . . . . . . . . 109

7.4 Upper rotor and lower rotor tip pitch deflections for coaxial and full vehicle CFD/CSD coupling at 150 knots. . . . . . . . . . . . . . . . 114

7.5 Upper rotor and lower rotor tip flap deflections for coaxial and full vehicle CFD/CSD coupling at 150 knots. . . . . . . . . . . . . . . . . 114

7.6 Upper and lower rotor tip flap and pitch deflections for coaxial and full vehicle CFD/CSD coupling at 150 knots. . . . . . . . . . . . . . 115

7.7 Non-dimensional normal force distribution for upper rotor, rotating counter-clockwise. . . . . . . . . . . . . . . . . . 117

7.8 Comparison of upper rotor sectional normal force at 150 knots. . . . . 119 
7.9 Non-dimensional normal force distribution for lower rotor, rotating clockwise. . . . . . . . . . . . . . . . . . . 120

7.10 Comparison of lower rotor sectional normal force at 150 knots. . . . . . 121

7.11 Non-dimensional chordwise force distribution for upper rotor, rotating counter-clockwise. .

7.12 Comparison of upper rotor sectional chordwise force at 150 knots. . . 124

7.13 Non-dimensional chordwise force distribution for lower rotor, rotating

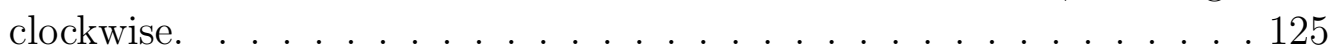

7.14 Comparison of lower rotor sectional chordwise force at 150 knots. . . 126

7.15 Non-dimensional pitching moment distribution for upper rotor, rotating counter-clockwise. . . . . . . . . . . . . . . 127

7.16 Comparison of upper rotor sectional chordwise force at 150 knots. . . 128

7.17 Non-dimensional pitching moment distribution for lower rotor, rotating clockwise. . . . . . . . . . . . . . . . . . . . . . . 129

7.18 Comparison of lower rotor sectional pitching moment force at 150 knots. 130

7.19 Upper and lower rotor flap bending moment obtained using coaxial and full vehicle CFD/CSD coupling at 150 knots. . . . . . . . . . . . 131

7.20 Upper and lower rotor chord bending moment obtained using coaxial and full vehicle CFD/CSD coupling at 150 knots. . . . . . . . . . . . 131

7.21 Time history of the upper and lower rotor torsion bending moment obtained using coaxial and full vehicle CFD/CSD coupling at 150 knots. 132

7.22 Convergence history for propeller power and collective. . . . . . . . . 135

8.1 Helicopter components and isolated parts interactional aerodynamic study . . . . . . . . . . . . . . . . . . 137

8.2 Aerodynamic interactions effects on the upper rotor. . . . . . . . . . 141

8.3 Pressure above the lower rotor at the disk plane of the upper rotor at 150 knots. . . . . . . . . . . . . . . . . 142

8.4 Upper rotor hubloads for various configurations shown in Fig. 8.2 (a) at 150 knots. . . . . . . . . . . . . . . . . . . . . 142

8.5 Aerodynamic interactions effects on the lower rotor at 150 knots. . . . 145

8.6 The difference between the coaxial rotor system and the lower rotor, the coaxial airframe combination and coaxial rotor system, helicopter and coaxial airframe combination at 150 knots. . . . . . . . . . . . . 146 \begin{tabular}{ll}
8.7 Lower rotor hubloads for various configurations shown in Fig. & 8.5 (a) \\
\hline at 150 knots. . . . . . . . . . . . . . . . . . . . . 147
\end{tabular}

8.8 Effect of blade passage on the pressure over the airframe 150 knots. 152

8.9 The time-series and harmonic amplitudes of the airframe spanwise at 150 knots. . . . . . . . . . . . . . . . . 153

8.10 The time-series and harmonic amplitudes of the airframe side force at 150 knots. . . . . . . . . . . . . . . . . . . . 154

8.11 The time-series and harmonic amplitudes of the airframe normal force at 150 knots. . . . . . . . . . . . . . . . . . . 155

8.12 The time-series and harmonic amplitudes of the airframe roll moment, positive starboard side up, about the center of lower hub at 150 knots. 156 
8.13 The time-series and harmonic amplitudes of the airframe pitching moment, positive nose up, about the center of lower hub at 150 knots. 157

8.14 The time-series and harmonic amplitudes of the airframe yaw moment, positive counter-clockwise, about the center of lower hub at 150 knots. . . . . . . . . . . . . . . . . . . . 158

8.15 Auxiliary propeller airloads at 150 knots. . . . . . . . . . . . . . . . . 161

B.1 CSD body-axis frame of reference used for trim procedure. . . . . . . 170

B.2 CFD global axis, utilized for describing CFD airframe airloads. . . . . . 170

B.3 Reference frame utilized for the non-dimensional deformed airloads, used for delta coupling. . . . . . . . . . . . . . . . . . . . . 171

B.1 Iso-surfaces of q-criterion, shown for $q=6 \mathrm{e}-4$, colored by the nondimensional vertical velocity, for 50 knot forward flight case. . . . . . 172

B.2 Iso-surfaces of q-criterion, shown for $q=6 \mathrm{e}-4$, colored by the nondimensional vertical velocity. The right column shows the view of from the top, while the left column shows the view from the starboard side. . . . . . . . . . . . . . . . 173

C.1 Iso-surfaces of q-criterion, shown for $q=6 \mathrm{e}-4$, colored by the nondimensional vertical velocity. . . . . . . . . . . . . . . . 174

C.2 Top view of the iso-surfaces of q-criterion, shown for $q=6 \mathrm{e}-4$, colored by the non-dimensional vertical velocity. . . . . . . . . . . . . . 175

D.1 Auxiliary propeller collective sweep for isolated and installed propeller at various forward flight speeds. . . . . . . . . . . . . . . . 176 


\section{Nomenclature}

$\begin{array}{ll}U_{T} & \text { tangential velocity } \\ r & \text { dimensional radial position } \\ \psi & \text { rotor azimuth, degrees } \\ V_{\infty} & \text { Free-stream velocity } \\ C_{n} M^{2} & \text { non dimensional deformed frame normal force } \\ C_{m} M^{2} & \text { non dimensional deformed frame pitching moment } \\ C_{c} M^{2} & \text { non dimensional deformed frame chordwise force } \\ n P & \text { Per rotor revolution } \\ r / R & \text { Non dimensional blade radial station } \\ \rho & \text { Density } \\ \theta_{0}, \theta_{1 c}, \theta_{1 s} & \text { Main rotor collective and cyclic pitch controls } \\ N_{b} & \text { Number of rotor blades (per rotor) } \\ R & \text { Rotor radius } \\ M_{\infty} & \text { Free-stream Mach number } \\ x_{B}, y_{B}, z_{B} & \text { Body frame axes } \\ \eta & \text { Efficiency factor } \\ A R & \text { Aspect ratio } \\ a_{0} & \text { Lift curve slope } \\ C_{D_{i}} & \text { Lift induced drag } \\ E, F, G & \text { Flux Vectors } \\ u, v, w & \text { Flow velocity components } \\ \gamma & \text { Ratio of specific heats } \\ p & \text { Pressure } \\ e & \text { Total energy }\end{array}$

\section{List of Abbreviations}

CA Comprehensive Analysis

CCW Counter Clockwise Rotation

CW Clockwise Rotation

CFD Computational Fluid Dynamics

CSD Computational Structural Dynamics

RANS Reynolds-Averaged Navier-Stokes

SA Spalart-Allmaras

X2TD X2 Technology Demonstrator

GRP Sikorsky Generalized Rotorcraft Performance Methodology

LOS Lift-Offset

RPM Revolutions per minute

CG Center of gravity

SMR Single main rotor 


\section{Introduction}

\subsection{Background and Motivation}

The objective of this research is to expand the fundamental understanding of the interactional aerodynamics of a high-speed coaxial compound helicopter. A fundamental understanding of the interactional aerodynamics is critical for the design of the next generation vertical lift vehicles. The next generation multi-role aircraft designs, such as the coaxial compound configurations shown in Fig 1.1 and tilt-rotors, consist of various components that function together to achieve highmaneuverability, high-speed cruise and efficient hover. A typical single main rotor helicopter, shown in Fig 1.2 , is not capable of achieving forward flight speeds exceeding 170 knots due to various limitations that arise during the high-speed flight regime [7].

The two dominant limitations that prevent single main rotor helicopters from achieving high-speed flight are the compressibility effects on the advancing side and the reverse flow region on the retreating side, shown in Fig.1.3. The edgewise velocity at a blade cross-section can be described using equation Eq1.1. The edgewise velocity at the blade is a function of the rotational speed of the rotor and the freestream velocity. The rotational speed of the rotor increases towards the tip. On 


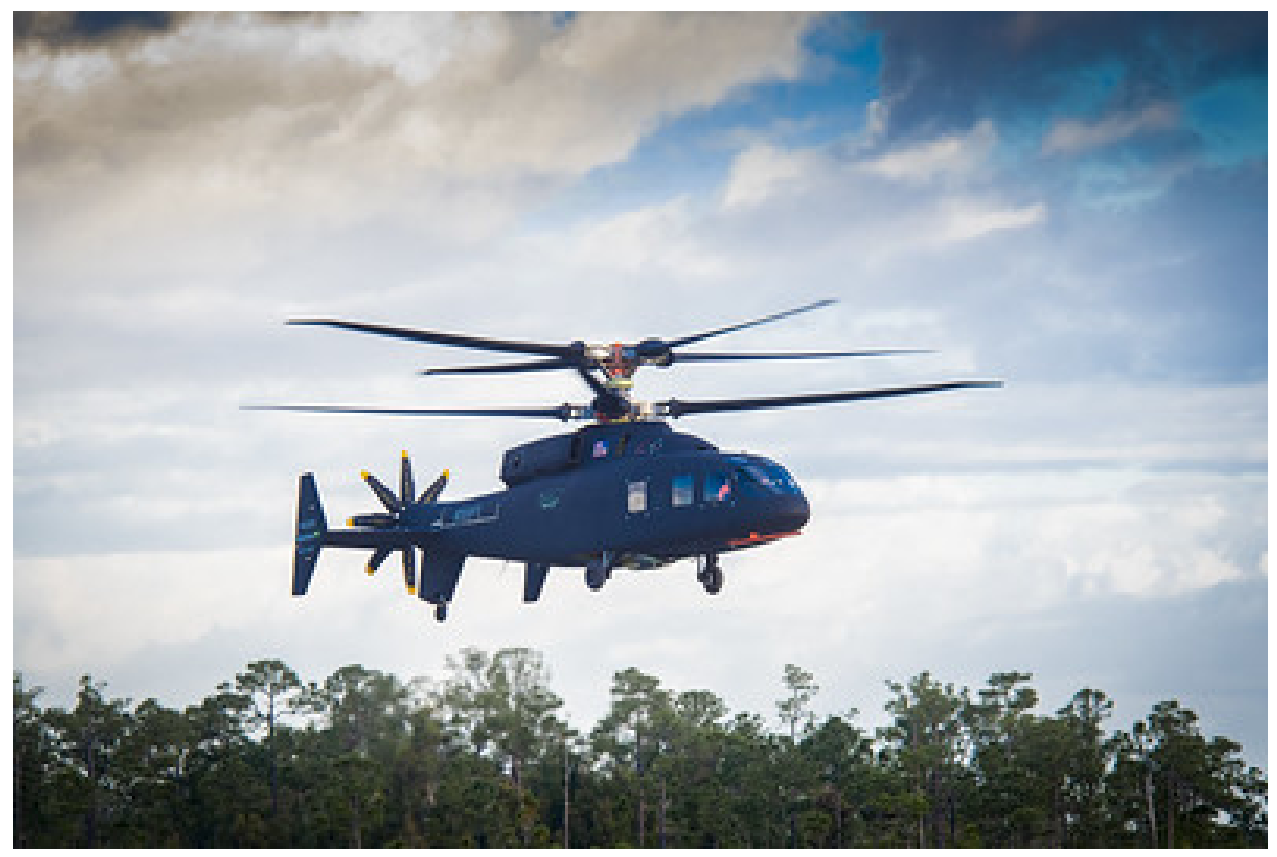

Figure 1.1: A high-speed coaxial compound helicopter, Sikorsky-Boeing SB $>1$ DEFIANT.

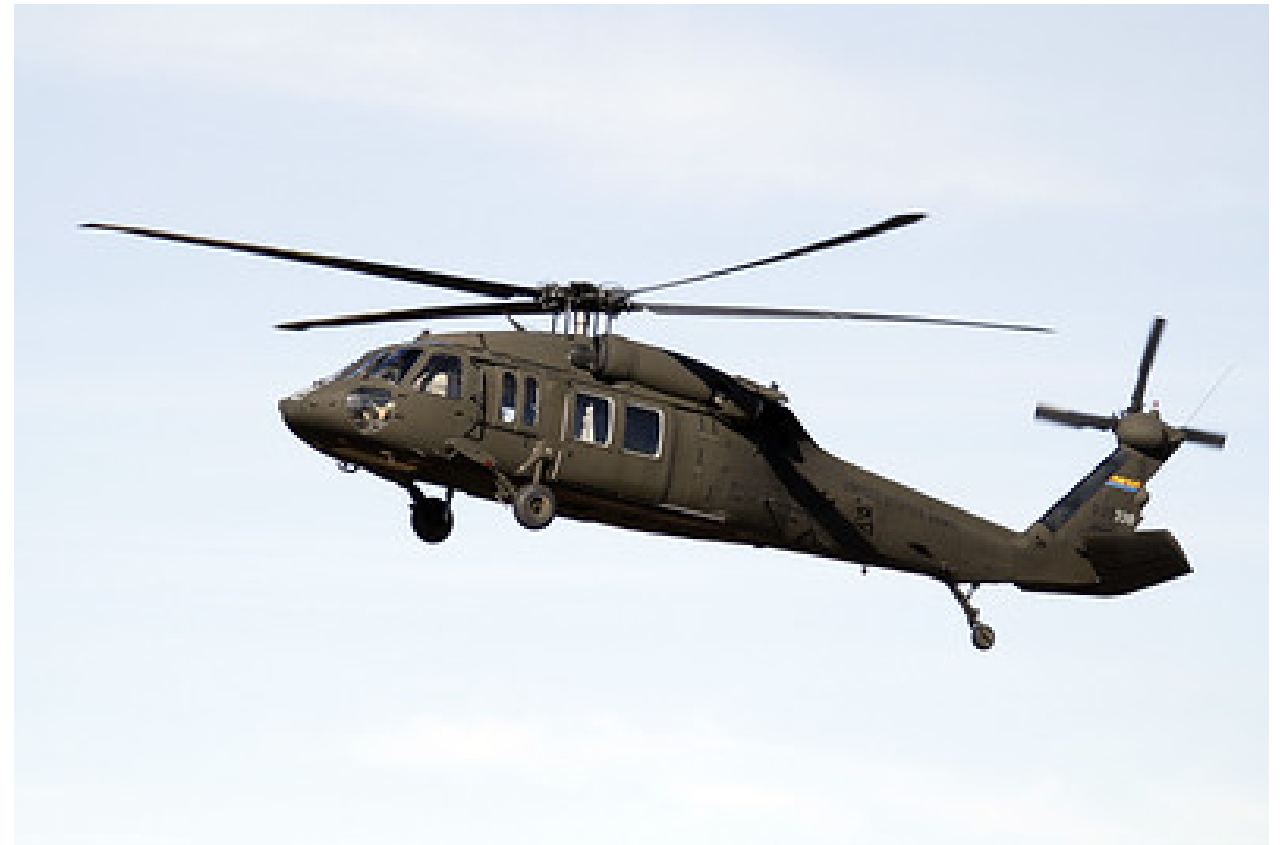

Figure 1.2: A conventional single main rotor helicopter, Sikorsky UH-60 Black Hawk. 
the advancing side, the blades are advancing into the free stream, resulting in the addition of vehicle airspeed and rotor rotational speed.

$$
\begin{gathered}
U_{T}(r, \psi)=\Omega r+V_{\infty} \cos \left(\alpha_{S}\right) \sin (\psi) \\
\mu=\frac{V_{\infty} \cos \left(\alpha_{S}\right)}{\Omega R}
\end{gathered}
$$

As the flight airspeed increases the addition of the rotational speed and the vehicle speed leads to the development of localized regions of transonic flow near the tip of the advancing blades. The compressibility effects include formation of shocks, resulting in loss of lift and increase in drag. On the retreating side, the blade advances with the free-stream resulting in the subtraction of the airspeed from the rotational velocity. On the inboard stations of the retreating blades the free-stream velocity is greater than the rotational velocity and the flow travels from the trailing edge to the leading edge of the blade. This phenomena is called reverse flow and the region on the blade where the flow is traveling from the trailing edge to the leading edge is called reverse flow region. Further along the blade, the rotational velocity becomes greater than the free-stream velocity and the flow travels in the nominal direction from the leading edge to the trailing edge. The radius of the reverse flow region is directly dependent on the advance ratio. The advance ratio of a helicopter is given in Eq.1.2. Thus, as the flight speed increases the advance ratio increases and the reverse flow region grows.

Inside the reverse flow region there is negative thrust and substantial drag increase. In order for the retreating side to produce thrust, the sectional blade 


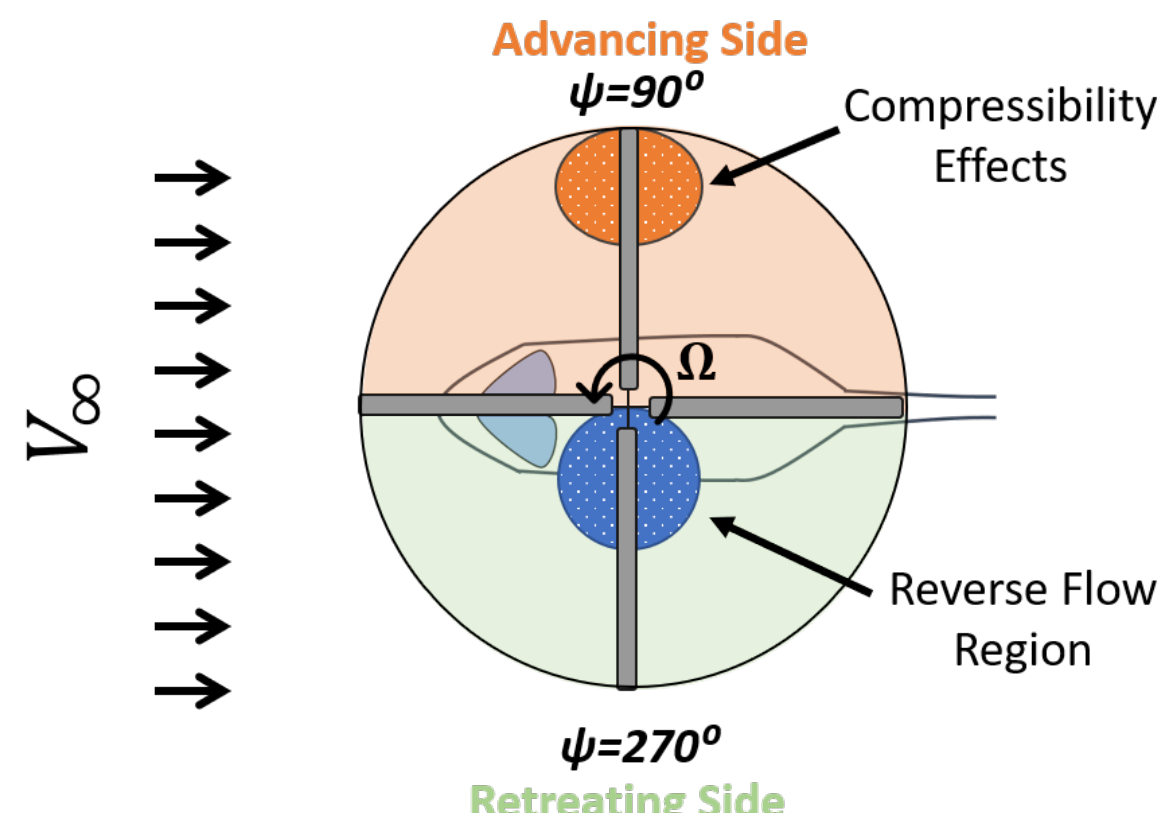

Figure 1.3: The limitations on a helicopter rotor which effect forward flight speed.

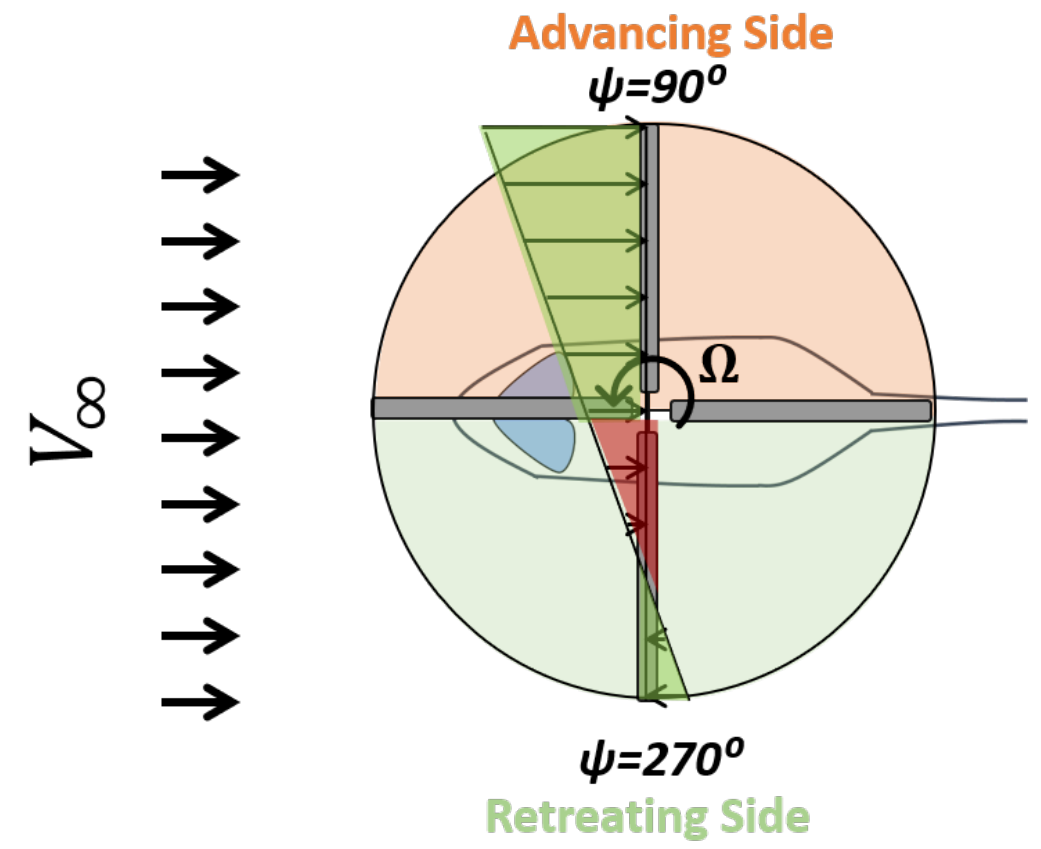

Figure 1.4: An example of edgewise velocity distribution around the rotor disk. 
angle of attack must increase. However, substantial increase in the sectional angle of attack leads to stall and flow separation. Thus, there is a limit on the available thrust produced by the retreating side of the rotor disk. The equilibrium of the roll moment, for a single main rotor helicopter, is enforced from the balance of the advancing side and retreating side roll moments. Therefore, the thrust produced by the advancing side is limited by the the thrust capabilities of the retreating side.

The need for high-speed helicopters and the limitations of the conventional single main rotor helicopters led to the innovation and the development of various viable multi-rotor, compound options including a coaxial compound helicopter. Over the past few decades substantial research was performed to realize the full potential of the coaxial compound helicopters as means of increasing the speed envelope of the rotary wing vehicles. The Sikorsky Advancing Blade Concept $\left(\mathrm{ABC}^{\mathrm{TM}}\right)$, discussed in Ref. [8], was developed to maximize the lift of the advancing side of the rotor disk and offload the retreating side by utilizing two, rigid counter-rotating coaxial rotors with lift-offset. High flap stiffness allows for the reduction of the rotor separation distance and maintenance of the required blade clearance. Unlike a single rotor configuration, where roll moment is achieved by the advancing and retreating sides of the rotor disk, the $\mathrm{ABC}^{\mathrm{TM}}$ configuration achieves the roll moment equilibrium by utilizing the advancing sides of both rotors. For an $\mathrm{ABC}^{\mathrm{TM}}$ rigid helicopter rotor, the thrust requirement is met while simultaneously maintaining vehicle rolling moment balance by exploiting the increased dynamic pressure on the advancing sides of each rotor to produce lift. Thus, most of the lift can be carried on the advancing side of the rotor, offloading the retreating side, and reducing the severity of retreating 
blade stall.

The Sikorsky X2 Technology Demonstrator (X2TD) successfully utilized the $\mathrm{ABC}^{\mathrm{TM}}$ concept and other design methodologies to reach speeds beyond 200 knots. In Ref. [5], Bagai discusses the methodology used for the design of the X2TD main rotor blades and the improvements that the new design had on the aerodynamic efficiency of the rotor at high speeds. The new design attributes included modern airfoils, non-uniform planform, variable twist gradients and thickness. An external auxiliary propeller provides the required propulsive force at high speeds, allowing for the decrease in main rotor RPM and alleviation of the compressibility effects on the advancing side [5]. The development of the Sikorsky X2 $2^{\mathrm{TM}}$ Technology Demonstrator (X2TD) showed that a coaxial helicopter has a capability to achieve higher maximum forward flight speeds, while still maintaining hover and low speed efficiencies $[1,5$, 6]. Further modifications and improvements to the Sikorsky X2TD coaxial aircraft have led to the evolution and the new development of the Sikorsky S-97 Raider Demonstrator and the Sikorsky-Boeing SB>1 DEFIANT, shown in Figs 1.5(b), (c) and (d), respectively.

The flow field around the helicopter is complex due to the aerodynamic interactions between various components and their wakes. The addition of the second rotor and the auxiliary propeller further increases the complexity of the flow field around the vehicle. Similarly to a conventional single main rotor helicopter, there exist aerodynamic interactions between the rotor and the fuselage as well as the blades and the trailing vortices. Additional interactions arise between the stacked rotors. The performance of the empennage and the auxiliary propeller is affected 


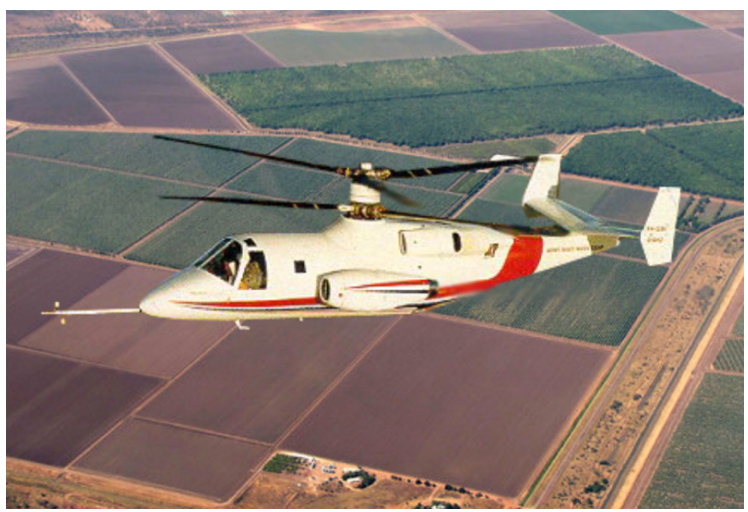

(a) Sikorsky S-69/XH-59A.

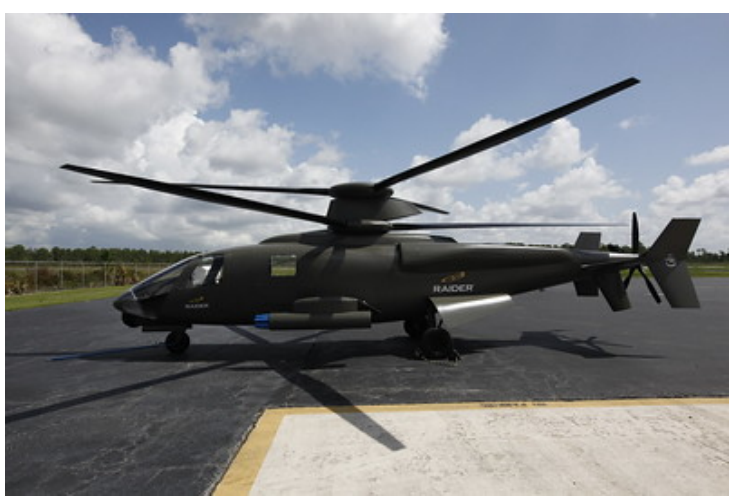

(c) Sikorsky S-97 Raider Demonstrator.

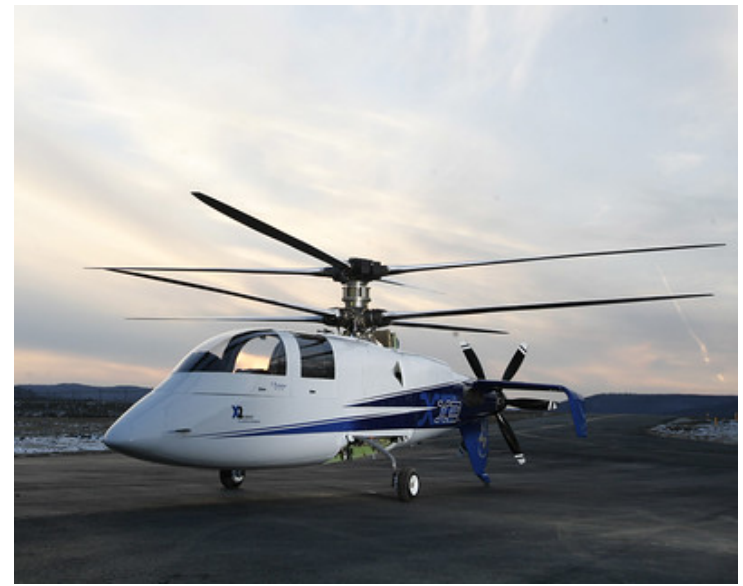

(b) Sikorsky X2 Technology Demonstrator.

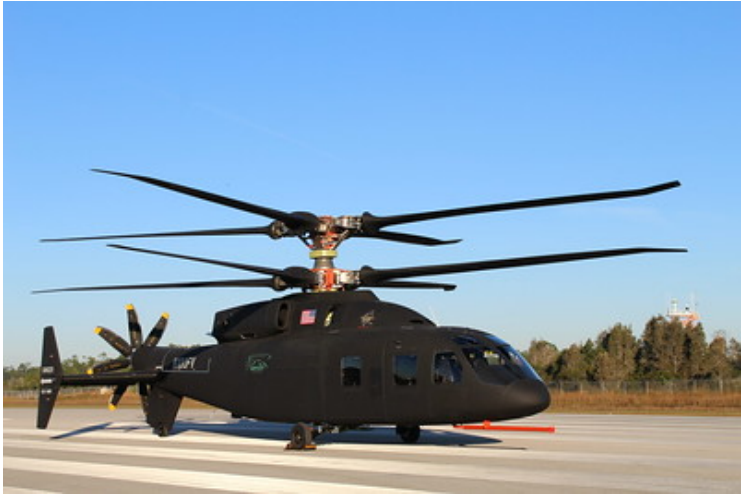

(d) Sikorsky-Boeing SB>1 DEFIANT.

Figure 1.5: Evolution of the Sikorsky coaxial aircraft. 
by the fuselage and rotor wake. Often times these components require additional modifications in order to function in the complex environment. Thus, thorough understanding of these interactions and their implications on the performance and the vibrations is crucial for the design and the testing of the aircraft. The interactional aerodynamics between the vehicle components must be considered in order to accurately predict the rotor performance, vehicle vibrations and aeroelastic blade response. Advanced simulations and experimental techniques are necessary to accurately model the interactional aerodynamics and predict stability and performance of the next generation vehicles.

The prediction of rotorcraft loads is inherently an coupled aeroelastic problem that requires precise modeling of the aerodynamics and structural dynamics of the vehicle, which are interdependent [9]. The trim solution and the modeling of the rotor blade structural dynamics is performed in a comprehensive analysis (CA) solver.

Numerous comprehensive analysis solvers as well as other special purpose codes have been developed over the years in industry, government and academia to study rotorcraft systems: CAMRAD [10], RCAS [11], DYMORE [12], UMARC [13], CHARM [14], PRASADUM [15], etc. A typical comprehensive analysis solver relies on the low-fidelity aerodynamic models to predict the blade sectional aerodynamic loads. These low-order aerodynamic models are incapable of accurately predicting the dynamic stall effects on the retreating side and 3D compressibility effects on the advancing side. The inaccuracies in the predictions of the airloads tend to propagate into the calculation of the structural blade loads [16]. The correction of the 
CSD airloads with the high-fidelity CFD airloads, known as the CFD/CSD coupling, improves airloads prediction which improves the accuracy of the structural blade loads. Over the past few decades the CFD/CSD coupling methodology has become a trusted tool in the rotorcraft community for the analysis of the complex rotorcraft problems.

\subsection{Summary of Previous Work}

Numerous efforts have been made to analyze and understand the high-speed coaxial compound helicopter design and the influence of aerodynamic interactions on the blade loads, the performance and the vibrations. Quite a few experimental studies were performed to analyze the performance of coaxial configurations in hover and level flight. However, the experimental studies do not provide detailed information regarding unsteady sectional airloads. Various studies focused on studying the rotor-rotor aerodynamics utilizing CFD/CSD coupling methodology to correct the low order rotor airloads within the comprehensive analysis solvers. Often times these studies only included isolated coaxial rotor system in the CFD simulations, without the airframe. Other studies focused on simulating full vehicle in the CFD and exchanging only the rotor airloads in the CFD/CSD coupling procedure. This work focuses on extending the CFD/CSD methodology to correct the airframe and auxiliary propeller airloads in the CSD solver, as well as to trim the auxiliary propeller. The objective of the extended methodology is to obtain high-fidelity full vehicle trim solution and to gain fundamental understanding of the full vehicle 
interactional aerodynamics for a high-speed coaxial helicopter. The following subsections will present selected prior works related to the experimental studies and modeling of interactional aerodynamics on in the rotary-wing vehicles as well as the high-fidelity numerical studies pertaining high-speed coaxial compound helicopters.

\subsubsection{Experimental Studies}

Numerous experimental studies have been performed to investigate the efficiency of the coaxial rotor system in hover. Most of these experimental studies focused on quantifying the performance of the coaxial rotors in hover. Harrington (1951) performed wind tunnel tests in the Langley full-scale wind tunnel to inves-

tigate the static-thrust performance of a coaxial rotor system in hover [17]. The results from the wind tunnel tests compared well with the theoretical calculations for a single main rotor of equivalent solidity. McAlister and Tung (2006) measured the hover performance of a small-scale coaxial rotor, varying the rotor separation distance and the proximity to the ground [18]. In 2014, Cameron et al. built and conducted hover experiments for a Mach-scaled rigid coaxial rotor system. The dynamic hub loads and the pushrod loads were compared for a single rotor, two-bladed and four-bladed coaxial rotor configurations [19].

Limited number of experiments have been performed for coaxial rotors in forward flight and only few experiments included the full vehicle. Dingeldein (1954) performed wind tunnel tests and quantified the power requirements of coaxial and tandem rotor systems in static thrust and level flight conditions [20]. The per- 

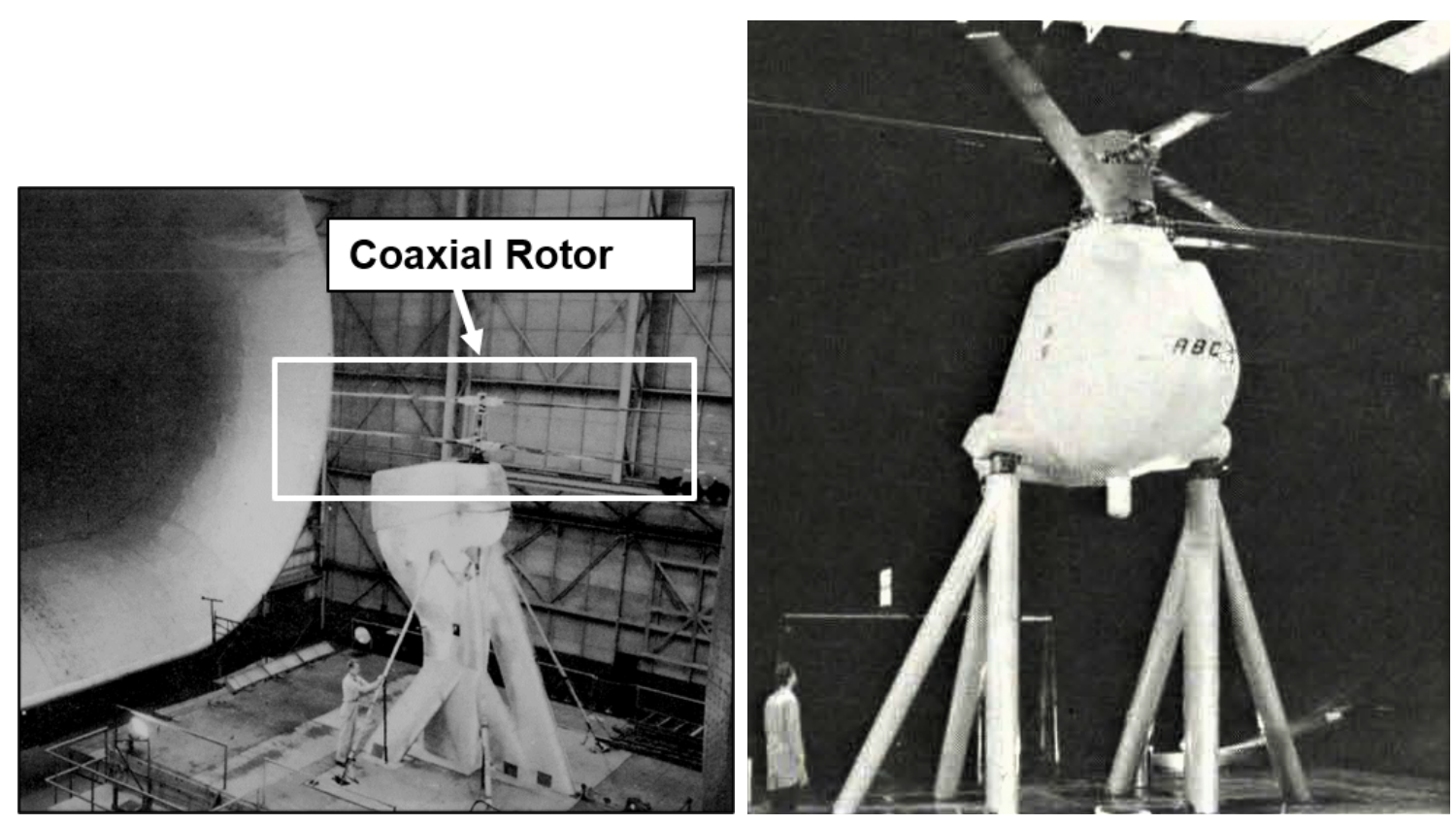

(a) Full-scale Harrington coaxial rotor in the Lan- (b) Full-scale rigid (ABC) coaxial rotor system gley wind tunnel 17 in NASA Ames 40- by 80-Foot wind tunnel 21]
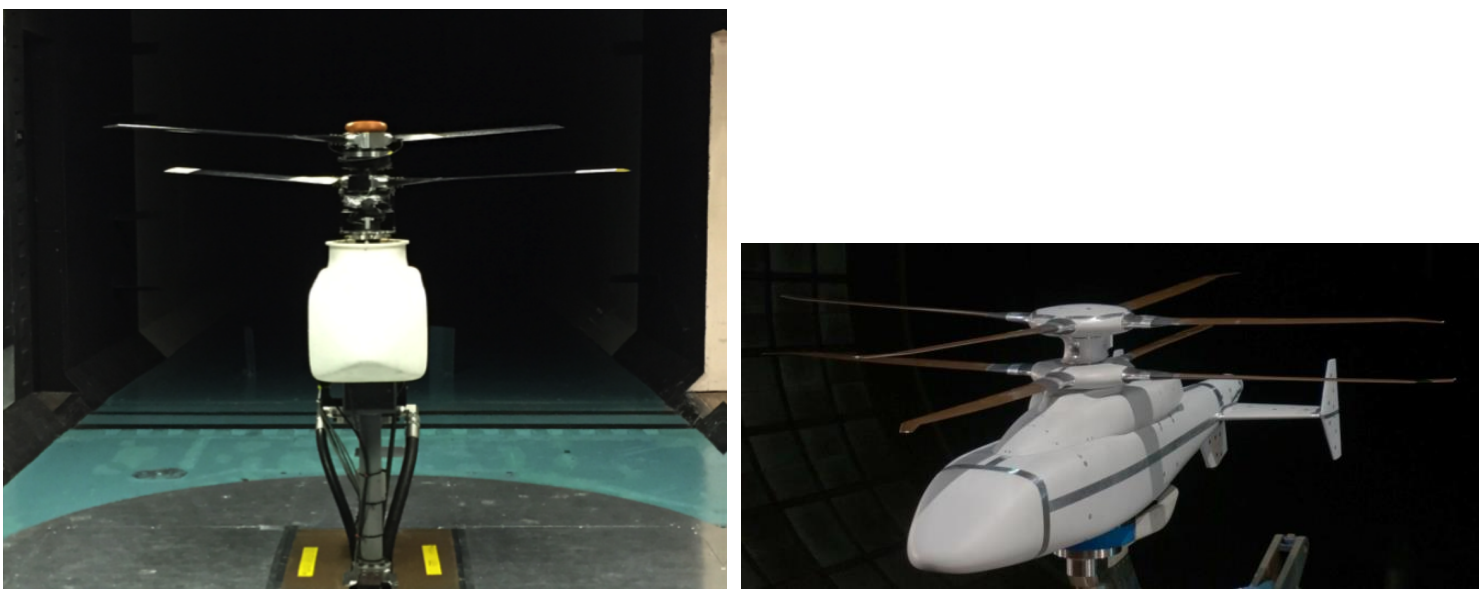

(c) Rigid two-bladed coaxial rotor in the Glenn (d) A 1/5 scale compound coaxial helicopter L. Martin wind tunnel 19$]$ tested in the NFAC wind tunnel 26

Figure 1.6: Experimental testing of coaxial rotor configurations. 
formance of the multi-rotor configurations was compared with an equivalent single rotor and theoretical calculation. The experiments showed that the coaxial rotor in forward flight required more power than a single rotor with equivalent solidity. Performance data was obtained for an $\mathrm{ABC}$ coaxial rigid rotor system during the wind tunnel tests at the 40- by 80-Foot NASA Ames wind tunnel [21], [22]. Felker (1981) described the testing of the full-scale coaxial compound X-H59A helicopter in the 40- by 80-Foot NASA Ames wind tunnel [23]. The wind tunnel tests were performed for the full vehicle configuration as well as the isolated components and the component combinations. Cameron and Sirohi (2016) performed wind tunnel tests in the Glenn L. Martin wind tunnel for a rigid untwisted two-bladed coaxial rotor 24]. The blade clearance, steady and vibratory hubloads were measured in that experiment. Lorber et al. presented results from a wind tunnel test of a scaled compound coaxial helicopter 25. The forces and moments on various helicopter components were measured. Figure 1.6 shows various experiments performed for a coaxial rotor configurations.

Only limited information is available regarding the flight tests performed for coaxial compound helicopters. Most of the data from the flight tests has not been publicly released. The XH-59A flight test program, spanning from 1973 to 1981, was focused on demonstrating the feasibility of the XH-59 coaxial compound helicopter configuration [27]. The Ka-32 helicopter flight tests were conducted by the to investigate the coaxial rotor wake using smoke flow visualizations and high-speed imagery [28]. The Sikorsky X2 Technology Demonstrator flight tests, conducted between 2008 and 2011, showed that the high-speed limitations can be overcome 
with a compound coaxial helicopter [6].

The aforementioned wind tunnel and flight tests provide useful knowledge regarding the performance of the coaxial rotor system and the time-averaged loads experienced by the vehicle. However, substantial instrumentation and complex setups are required to obtain the unsteady loads, aerodynamic as well as structural, along the rotor blade span at various azimuthal locations.

\subsubsection{CFD/CSD Simulations - SMR Configurations}

Extensive work has been performed in the area of CFD/CSD coupling applied to single main rotor configurations [29]. The availability of flight test data and experimental data for the UH-60A and HART-II configurations allowed for validation of the CFD/CSD coupling methodologies and expansion of these methodologies to other single main rotor configurations. Coupling high-fidelity rotor CFD and CSD significantly improved predictions of the airloads and structural loads for a single main rotor configurations for a range of operating conditions.

Datta et al. showed that CFD/CSD coupling improved the prediction of the unsteady transonic pitching moment at high forward flight speeds for a UH-60A rotor [30]. Improvement in the UH-60A torsional load predictions, using CFD was also observed [16]. Potsdam showed that CFD/CSD accurately capture blade-vortex interactions for UH-60A in a low speed flight [9]. However, these rotor CFD/CSD studies included only the rotor in the CFD simulations and neglecting the airframe.

Over time the capabilities of the CFD/CSD coupling methodologies have increased 
dramatically to include the whole vehicle in the CFD simulations utilized for the CFD/CSD studies. Numerous attempts have been made to perform full vehicle CFD/CSD coupling studies of single main rotor helicopters.

Various researchers have successfully studied the acoustics and interactional aerodynamics of the HART-II configuration using CFD/CSD coupling methodology [2], 31], 32], 33]. The HART-II case consists of a single main rotor mounted on top of a fuselage-shaped fairing supported by a sting, shown in Fig.1.7(a). A typical mesh used in the CFD simulations, representing the HART-II configuration, is shown in Fig.1.7(b). For the HART-II configuration, the presence of the fuselage effected the blade vortex interactions on the advancing side of the rotor disk. The UH-60A simulations have also increased in complexity. The CFD/CSD simulations were modified to include the UH-60 airframe and in some cases the tail rotor 34.

Embacher et al. (2010) performed a helicopter CFD/CSD feasibility study, coupling in the time-averaged airframe airloads in addition to the unsteady rotor airloads for the Eurocopter EC145 helicopter [35]. The EC145 helicopter consisted of a single main rotor, a tail rotor, airframe and skids. Embacher et al. showed that there were significant fluctuations in airframe pitching moment and lateral force, which affected the final trim state of the helicopter.

Lorber, Min and Zhao (2019) analyzed the unsteady tail interactions and the rotor-fuselage flow field, on the S-92 helicopter configuration, using STAR-CCM+ and CREATE-AV Helios CFD simulations [26]. The rotor was trimmed, using RCAS, to match the rotor flapping from the experiments. The authors showed that the blade tip vortices and the retreating blade stall vortices introduce additional 


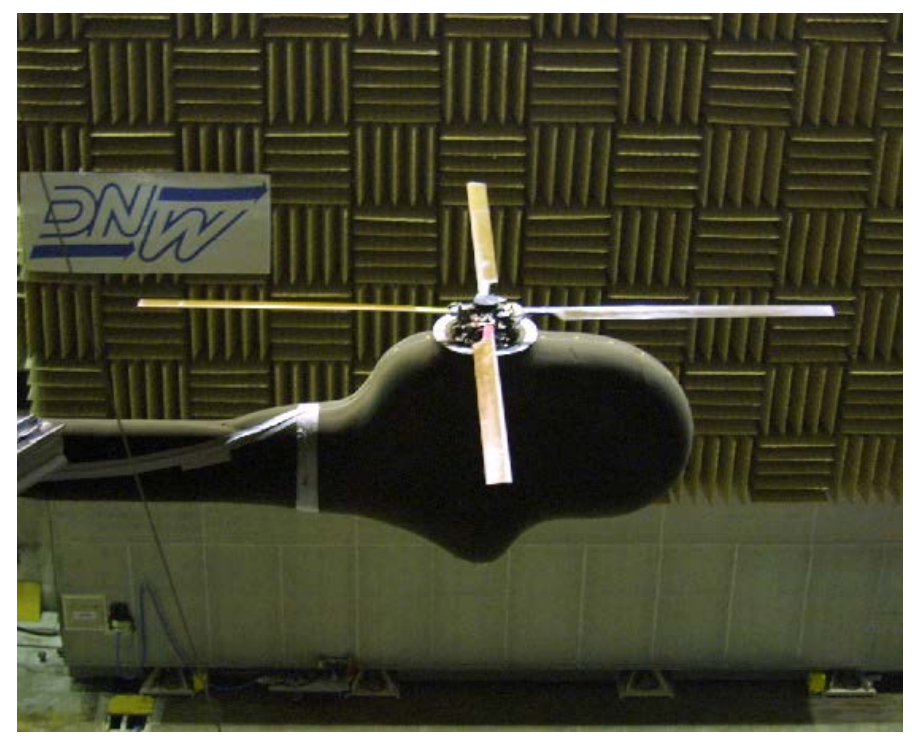

(a) Hart II experiment.

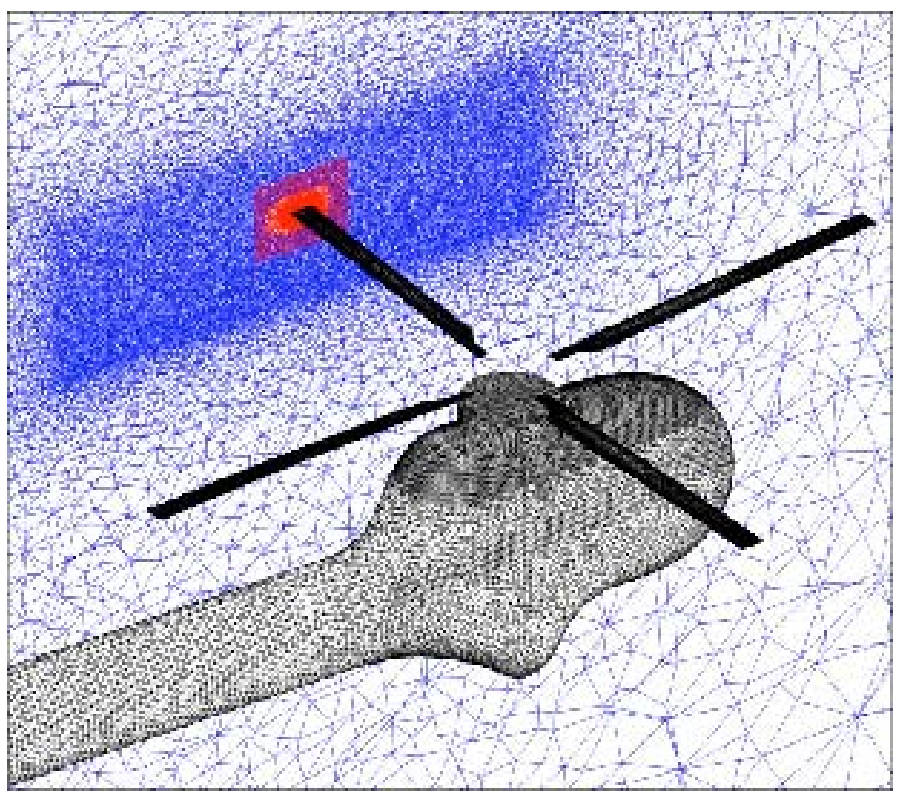

(b) Hart II mesh.

Figure 1.7: Hart II CFD/CSD study presented in Ref. [2] 
harmonics into the empennage loads. This study did not couple the high-fidelity CFD airframe airloads back into the CSD solver.

Roget et al. (2019) studied the effect of performing full vehicle CFD/CSD coupling for a single main rotor helicopter, UH-60A, using CREATE-AV Helios and RCAS [36]. Roget demonstrated the feasibility of performing full vehicle CFD/CSD coupling and correcting the low order airframe airloads with high-fidelity CFD results.

\subsubsection{CFD/CSD Simulations - Coaxial Rotor Configurations}

The high-fidelity CFD/CSD coupling studies pertaining to lift-offset coaxial rotors, especially in high-speed forward flight, are not as common as the SMR CFD/CSD studies described above. Only limited experimental data is available for the lift-offset coaxial rotor making it more challenging to validate the CFD/CSD coupling methodologies for coaxial rotors. The complexity that arises from the addition of multiple rotors in both the CFD and CSD simulations also play a factor. Nevertheless, in the past few years various CFD/CSD studies were performed to study the aerodynamics of coaxial rotors.

Passe (2015) conducted a CFD/CSD study, utilizing CREATE-AV Helios and an in-house comprehensive analyzing code PRASADUM, to study interactional aerodynamics of a notional X2TD coaxial rotor system at 55, 100 and 150 knots 37. Passe showed that CFD/CSD captured an 8P impulsive change in normal force and pitching moment caused by the blade crossings. In this study the coaxial rotor was 
trimmed using propulsive trim methodology in PRASADUM and only the coaxial rotor was present in the CFD simulations. The CSD low order aerodynamic airloads were corrected using the high-fidelity CFD airloads. Klimchenko (2017) expanded this work to higher forward flight speeds, 200 and 225 knots 38.

Jacobellis et al. (2018) used a dynamic inflow model, Viscous Vortex Particle Method (VVPM) and the high-fidelity CFD/CSD coupling to predict the airloads on the coaxial rotor at a high-advance advance ratio of $\mu=0.66[3]$. A wind tunnel trim of the coaxial rotor was performed using RCAS. The iso-surfaces of vorticity from the CFD and the VVPM simulations are shown in 1.8. The physics-based models, VVPM and high-fidelity CFD, were able to captured the $8 \mathrm{P}$ blade-blade interactions. However, VVPM and dynamic inflow model rely on the tabulated 2D airfoil data and are unable to accurately predict the aerodynamic phenomena in the reverse flow region. The CFD simulations captured the effects of the reverse flow region on the airloads.

Jia and Lee (2019) utilized high-fidelity CFD/CSD coupling, CREATE-AV Helios coupled with RCAS, to study the acoustic signature of the coaxial helicopter at 100, 150 and 200 knots [39]. The study showed that there is an increase in impulsive sound-pressure level for coaxial rotor with lift-offset. Authors noted that the shaft angle was fixed at zero for this study and there may be variations in impulsive acoustics with different shaft angles.

Additional studies were performed using CFD/CSD methodology which included the full vehicle geometry in the CFD, without coupling in the airframe airloads into the CSD, applied to multi-rotor vehicles. Bhagwat et al.(2008) studied 

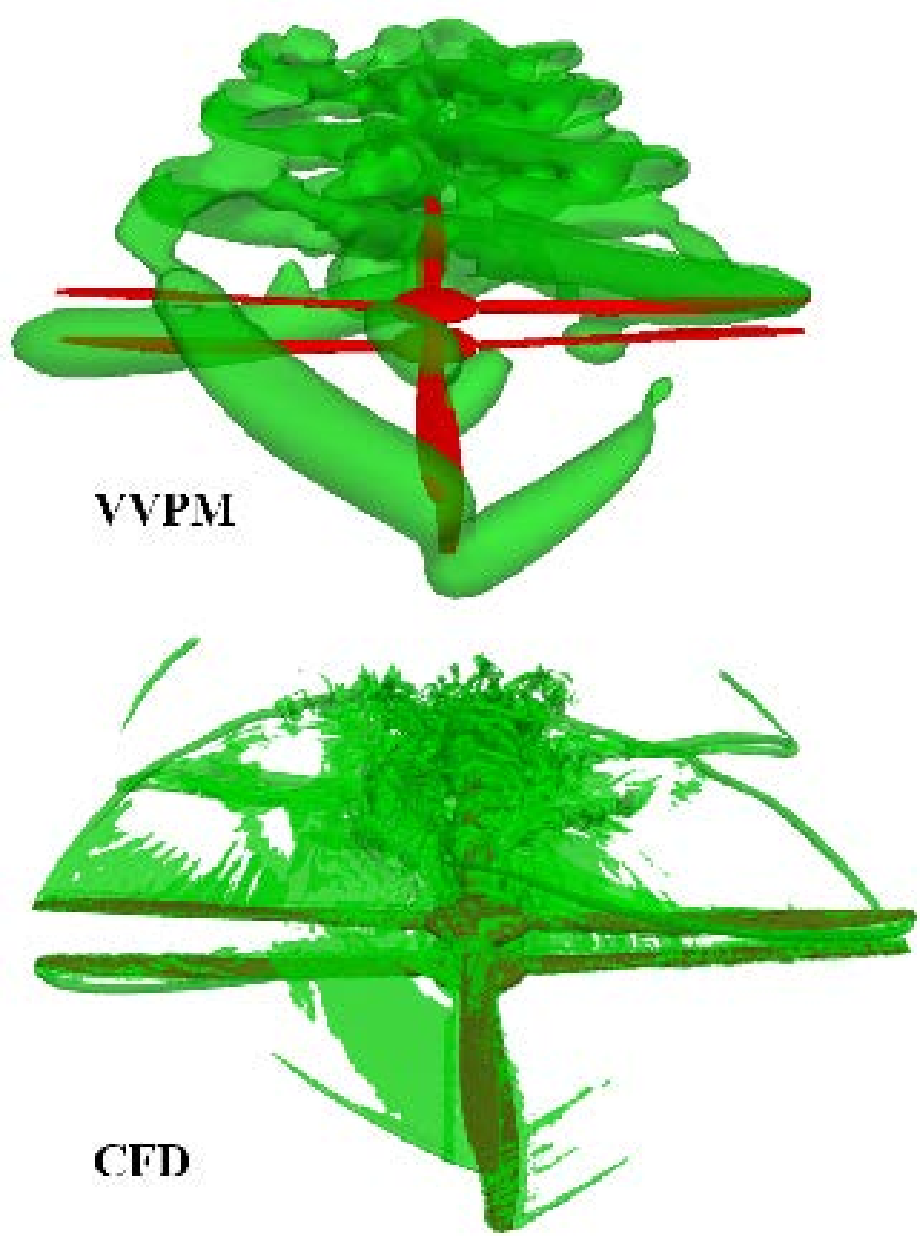

(a)

Figure 1.8: The iso-surfaces of vorticity from the CFD and VVPM simulations presented by Jacobellis et al. in Ref. [3].

airloads, blade loads and vehicle performance of a duel-rotor CH-47 helicopter using CFD/CSD coupling methodology [40]. The vehicle fuselage was modeled in the CFD simulations. Trim calculations and structural modeling was performed using RCAS, while the fluid dynamic simulations were performed using OVERFLOW. The comprehensive analysis airframe airloads were not corrected with the airloads from CFD solution.

Zhao, Brigley and Modarres (2019) compared high fidelity CFD/CSD vibra- 
tory hub loads and blade loads of the S-97 RAIDER, in low speed transition flight, to 2016 and 2017 flight test data [41]. The propeller was not operation and the propulsive force was generated by the rotors during these flight tests. The blade deflections were modeled using the Rotorcraft Comprehensive Analysis System (RCAS) [11]. Both rotors were trimmed to time-averaged vertical force and pitch/roll moments obtained from the actual flight test. Since previous studies showed that the flexibility of the airframe did not have significant effect on the vibratory loads, flexibility of the airframe was ignored and rotor hubs were independently connected to the ground. The CFD simulations included coaxial rotor system, rotor hubs and the fuselage (excluding the empennage and the pylon). Only airloads from the coaxial rotor were coupled with CSD. The authors correctly predicted the upper and lower rotor $3 \mathrm{P}$ blade normal bending moments but underpredicted the $5 \mathrm{P}$ blade normal bending which lead to the underprediction of the peak 4P hub moment.

Bowles et al. (2019) studied interactional aerodynamics of a full-vehicle, S-97 RAIDER aircraft, using a commercial unstructured solver (STAR-CCM+) [42]. The main rotor and the auxiliary propeller blades were modeled using the Virtual Blade Model (VBM) which is an embedded blade-element method model in STAR-CCM+. The simulation results were compared with wind-tunnel and flight test data. The presence of spinning hubs results in large increase in the unsteady yaw moment due to the coupling of lower rotor hub wake with pylon wake structures.

Anusonti-Inthra (2019) studied the effects of the fuselage on the performance of a conceptual coaxial compound rotorcraft at two moderate advance ratios, $\mu=0.234$ and $\mu=0.0 .292$, using loose CFD/CSD coupling methodology [43]. The CFD 
simulation included coaxial rotor system, rotor hubs, fuselage, canards, stabilizers and ducted fan shrouds. Rotor structural dynamics were modeled with RCAS. The rotor blade airloads from Helios/FUN3D were coupled to RCAS, airloads from the fuselage were not coupled between CFD and CSD. The study found that the presence of the fuselage significantly effected the lower rotor flap bending moment on the retreating side as well as the blade vertical load harmonics. However, the presence of the fuselage did not have significant effects on the final trim parameters.

The coaxial vehicle CFD/CSD studies mentioned above included the airframe geometry in the CFD simulations but did not correct the CSD airframe airloads with the high-fidelity airframe airloads from CFD. In 2016 Passe et al. performed a unique CFD/CSD study focused on studying the effect of the rotor-airframe interactional aerodynamics for a coaxial compound helicopter [44]. His objective was to couple in the time-averaged airframe airloads into the CSD. This CFD/CSD study showed that correcting the CSD airframe airloads with CFD airloads changed the final trim state of the coaxial rotor due to additional moments produced on the airframe by interactional aerodynamics. This study did model the auxiliary propeller in the CFD and neglected the auxiliary propeller in the CFD/CSD coupling.

\subsection{Contributions of Present Research}

The literature survey of the previous work shows that there is a substantial lack of high-fidelity CFD/CSD studies pertaining to the high-speed coaxial compound helicopter with lift-offset. Most of the studies discussed did not utilize full vehicle 
CFD/CSD methodology to predict the trim state and the performance of the vehicle. Only one study, Ref. [44], corrected the coaxial helicopter airframe CSD airloads with high-fidelity CFD airframe airloads. However, this CFD/CSD study did not account for interactional aerodynamics on auxiliary propeller. The present research will show the importance of correcting both, the airframe and propeller CSD airloads, with the high-fidelity CFD airloads and the effect of inter-component aerodynamics on the final trim state of the vehicle.

The objective of the present research is to extend the full vehicle CFD/CSD coupling procedure to a high-speed coaxial helicopter and to fill in the gap that exists in literature that pertains to interactional aerodynamics of the coaxial compound helicopter using high fidelity full vehicle CFD/CSD coupling procedure.

1. Develop full vehicle CFD/CSD coupling methodology for a high-speed coaxial compound helicopter. Include the correction of time-averaged airframe airloads and auxiliary propeller power in the comprehensive analysis. Simultaneously trim the propeller to mandatory power, correcting CSD propeller power with CFD power. Identify and analyze the effect of the interactional aerodynamics on the final trim state of the vehicle, structural blade deformation and hub loads.

2. Develop a fundamental understanding of interactional aerodynamics of a highspeed coaxial compound helicopter. Systematically compare the vehicle component airloads, in isolation and in installed configuration, to identify and analyze the effects of components on one another. 
3. Characterize the lift-offset high-speed coaxial rotor propulsive trim variable trends, airloads and structural deformation for a range of advance ratios. Understand the effect of lift-offset on the airloads and structural blade deformations.

The intent of this study is to develop the computational tools and gain fundamental insights into the complex aeromechanics of a modern lift-offset coaxial compound rotorcraft.

\subsection{Scope and Organization of Thesis}

The current chapter focused on giving the reader an overview of the limitation of a high-speed single main rotor helicopter and convincing the reader that the coaxial compound helicopter can overcome these limitation. The complex interactional aerodynamic environment that arises in the vicinity of the coaxial compound helicopter components was described. The high-fidelity numerical methodologies that are needed to accurately simulate the compound helicopter were introduced. Prior work which utilized the high-fidelity methodologies for single main rotor and a coaxial rotor was discussed.

Following this introduction, the second chapter describes the notional helicopter studied in the present research and gives physical description of the helicopter. The third chapter discusses the in-house computational structural dynamics solver, PRASADUM. The coaxial compound helicopter trim equations and the modeling of the structural blade dynamics are also discussed. The third chapter examines 
the computational fluid dynamics framework, CREATE-AV Helios, and discusses the individual solvers used in this study. It also gives a brief overview of the fundamental fluid dynamics equations that are solved. The fourth chapter discusses the conventional and the full vehicle CFD/CSD coupling methodology used for the research and provides validation for the CFD/CSD methodology using a well known UH-60A rotor case which was part of the UH-60A Airloads Workshop efforts.

The results are presented in three chapters, chapter five through chapter seven. The first part of the results, chapter five, focuses on studying the individual components of the vehicle at various speeds. The coaxial rotor system is analyzed at various speeds using conventional rotor CFD/CSD coupling methodology. The converged trim states, airloads and blade deformations are analyzed and compared for various speeds. The vehicle airframe is also analyzed at various speed and the force breakdown on various components of the airframe is shown. The next chapter, chapter six, focuses on comparing the full vehicle CFD/CSD coupling methodology with the rotor CFD/CSD coupling methodology. The final trim variables and rotor airloads are compared. The CSD predicted airframe airloads are also compared to the CFD predicted airframe airloads. The last results chapter, chapter eight, focuses on systematically increasing the complexity of the simulations to understand the fundamental aerodynamic interactions that occur between different vehicle components. Chapter nine summarizes the research, provides key conclusions and recommends future work. 


\section{Notional High-Speed Coaxial Compound Helicopter}

This chapter describes the helicopter configuration modeled in this work. The helicopter modeled in this study is a Sikorsky X2 Technology Demonstrator (X2TD), shown in Fig 2.1. The X2TD is a 6000-lb class compound coaxial helicopter designed to operate at high forward flight speeds greater than 200 knots. This helicopter configuration consists of a coaxial rotor system, airframe and an auxiliary propeller.

The parameters for the notional X2TD coaxial rotor system and the airframe used for this study were approximated by Passe in Ref. [45] to closely match the publicly available data from flight tests in Refs. [1], [5], [6]. Because the helicopter parameters modeled in this study were estimated from publicly available data, the helicopter is referred to as "notional" X2TD. The rotor swashplate, rotor hub fairings and the blade root cutout regions are not modeled in this study. The following sections will describe the components of the notional X2TD helicopter utilized in this study.

\subsection{Coaxial Rotor}

The X2TD coaxial rotor system consists of eight blades, four blades per rotor, with upper rotor rotating counter-clockwise and the lower rotor rotating clockwise. All eight blades are identical. The rotor parameters are given in the Table 2.1. The 


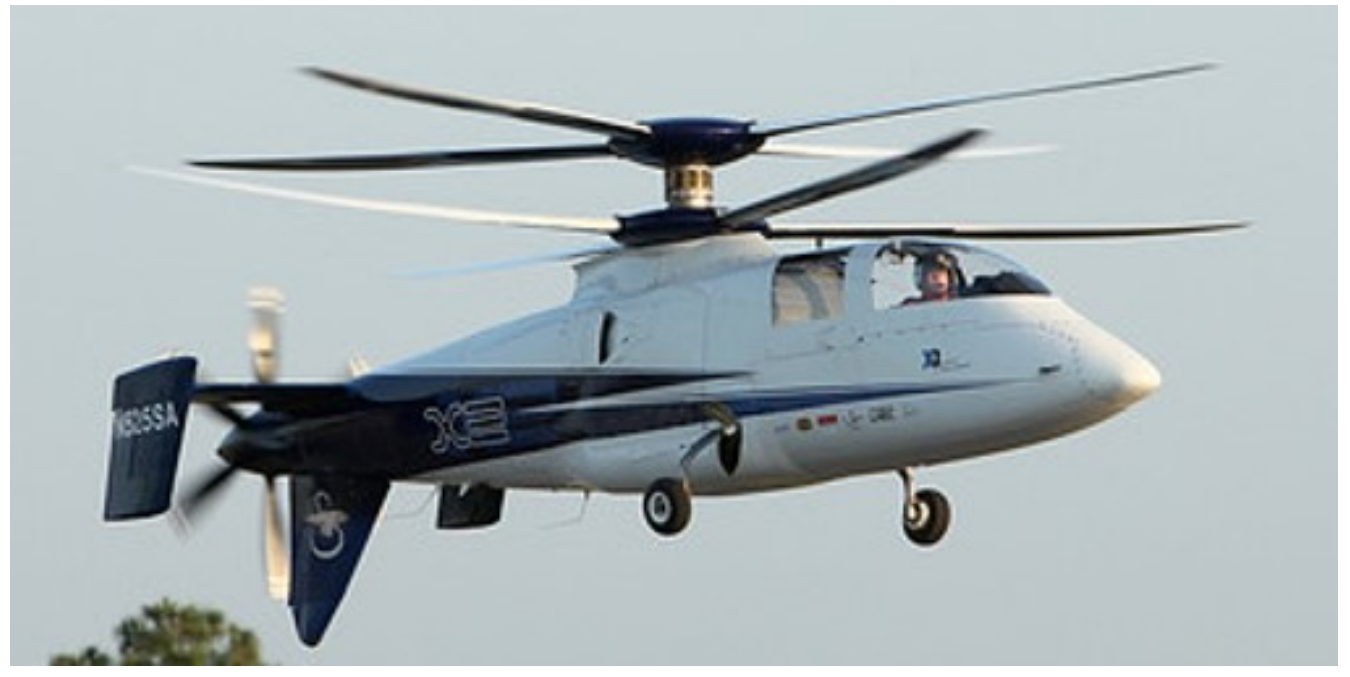

Figure 2.1: A flight test of the Sikorsky X2 Technology Demonstrator Ref. [4].

Table 2.1: The X2TD rotor system parameters

\begin{tabular}{l|c|c}
\hline \hline Parameter & Value & Units \\
\hline Number of rotors & 2 & \\
Number of blades per rotor & 4 & \\
Radius & 13.2 & $\mathrm{ft}$ \\
Rotor Vertical Separation & 1.5 & $\mathrm{ft}$ \\
Root chord & 0.687 & $\mathrm{ft}$ \\
Rotation Speed - Hover to 200 knots & 446 & $\mathrm{RPM}$ \\
Rotation Speed - 280 knots & 360 & $\mathrm{RPM}$ \\
\hline \hline
\end{tabular}


radius of the main rotor is 13.2 feet with root cutout of 1.5 feet. The rotor blades of the Sikorsky X2TD rotor were designed, using the Sikorsky Generalized Rotor Performance (GRP) methodology, to improve rotor aerodynamic performance at high-speeds without compromising hovering efficiency or low speed cruise characteristics [5].

The names of the airfoil sections utilized for the actual X2TD blade were not publicly released. Thus, only limited information is available about the X2TD airfoils and their selection. Reference [5] briefly discusses the airfoil selection process and compares the thickness of the X2TD rotor blade to the XH-59A rotor blade, shown in Fig 2.2. The airfoil cross-sections along the notional X2TD blade, shown in Fig 2.3(a), were selected in Ref. 45 to closely resemble the actual X2TD blade and to satisfy the blade design requirements discussed in Ref. [5]. The notional X2TD rotor blade, utilized in this study and shown in Fig.2.3(a), was assembled by Passe in Ref. [45].

The actual X2TD rotor blade consists of proprietary thick double-ended airfoil on the inboard sections of the blade [5]. At high-speeds, there is a large reverse flow region on the retreating side of the X2TD rotor disk, where the air travels from the trailing edge to the leading edge of the airfoil, resulting in separation and an increase in drag. Compared to a sharp trailing edge airfoil, the double-ended airfoil sees reduced reverse flow separation which minimizes the reverse flow penalties. A double-ended Sikorsky DBLN-526 rotorcraft airfoil was selected and modified for the in-board sections of the notional X2TD rotor blade. The modified DBLN-526 airfoil 


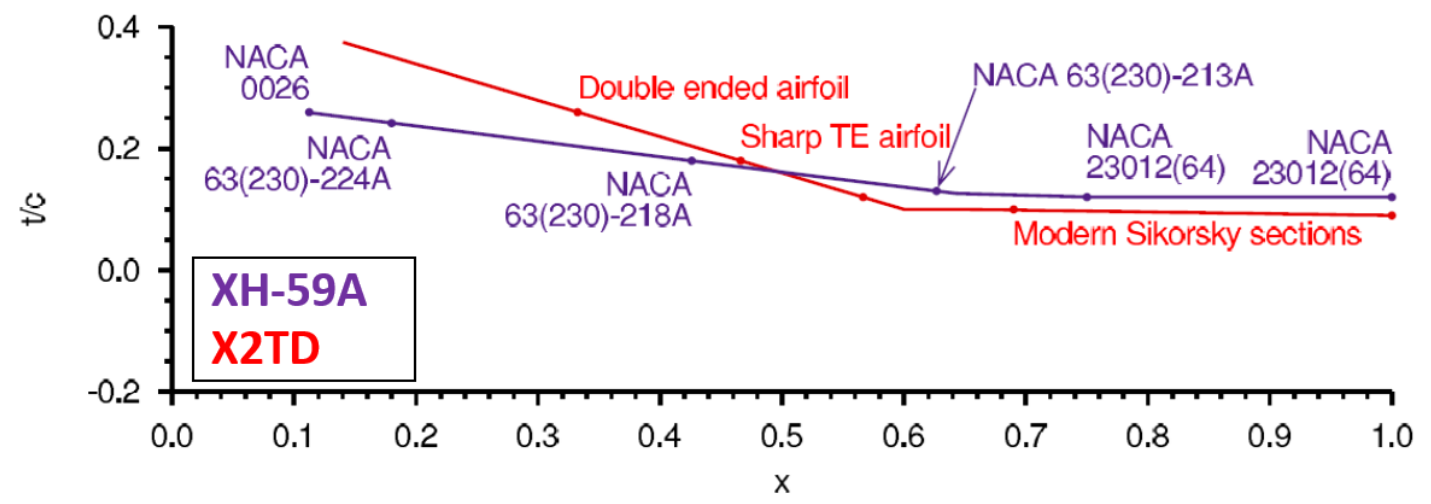

Figure 2.2: Sikorsky X2TD and XH59-A main rotor blade thickness distribution [5].

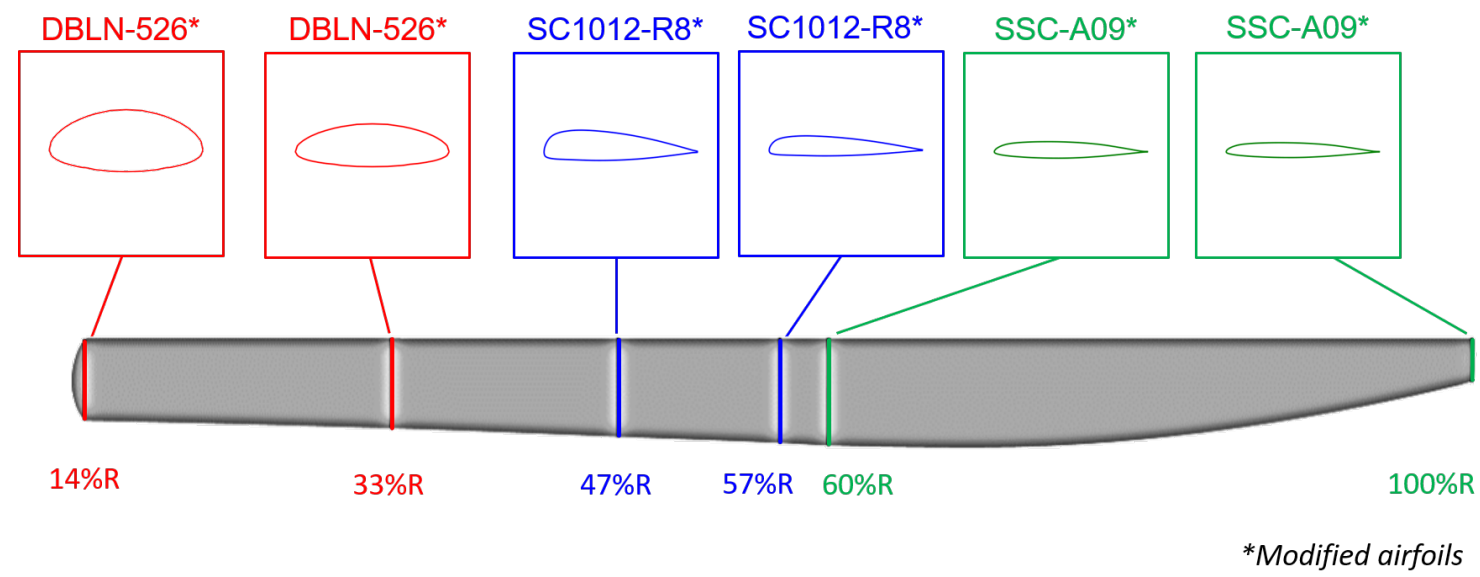

(a) Notional X2TD airfoil profiles.

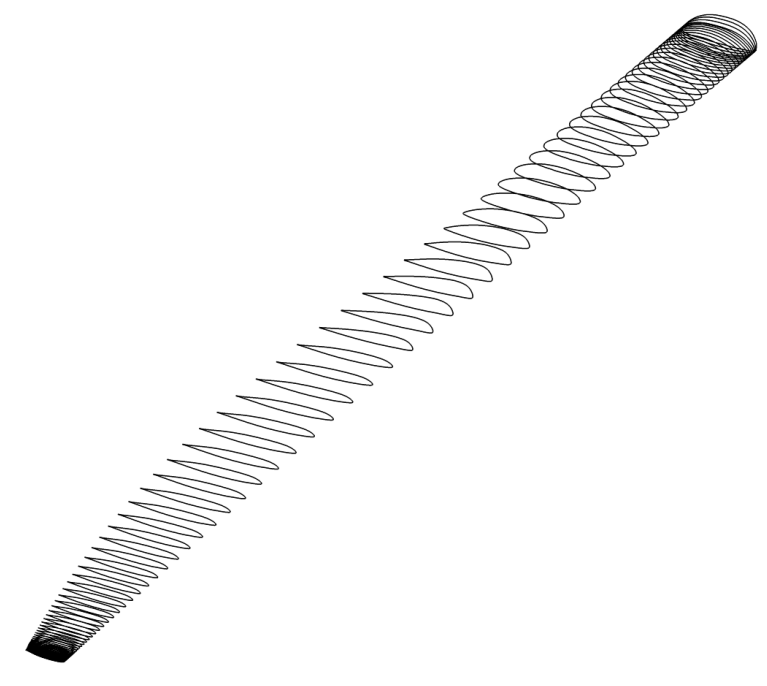

(b) Airfoil distribution.

Figure 2.3: Notional X2TD rotor blade. 
extends from the root to $33 \% \mathrm{R}$ of the rotor blade. An unspecified conventional sharp trailing edge airfoil was incorporated in transition from the double-ended airfoil at the root to the advanced transonic airfoils on the outboard stations of the actual X2TD rotor blade. The blade tip on the advancing side of the rotor disk operates in transonic flow during high-speed flight, which results in compressibility effects and performance penalties. A choice of airfoil cross-section is crucial in this region of the blade. An advanced transonic airfoil, Sikorsky SSC-A09, was chosen for the tip of the notional X2TD rotor blade, while a Sikorsky SC1012-R8 was selected for the transition region of the notional X2TD rotor to ensure smooth blend between the blunt and the sharp trailing edge airfoil profiles near the midspan.

Take note that Fig. 2.3(a) shows the start of true airfoil sections of the notional X2TD blade described above. In between the demarcation lines the airfoils are automatically interpolated, in the blade mesh generator, for smooth transition between the adjacent airfoil profiles, as shown in Fig,2.3.

Figure 2.4(b) shows the non-linear chord distribution along the span of the notional X2TD rotor blade. The rotor blade chord increases towards the outboard stations of the blade for $70 \% R$. The studies of the XH-59A blades have shown that the 2:1 taper ratio resulted in the high retreating blade drag on the inboard stations of the retreating blades. Therefore, the X2TD blades were designed with a non-linear taper and the redistribution of the blade area towards the outboard sections.

Figure 2.4(c) shows the bi-linear twist distribution of the notional X2TD rotor blade. The positive twist gradient, on the inboard stations the blade, reduces the 


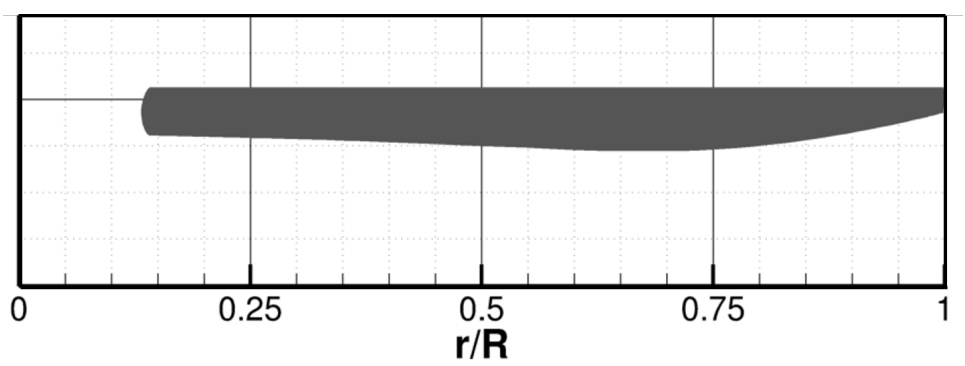

(a) Notional X2TD blade planform

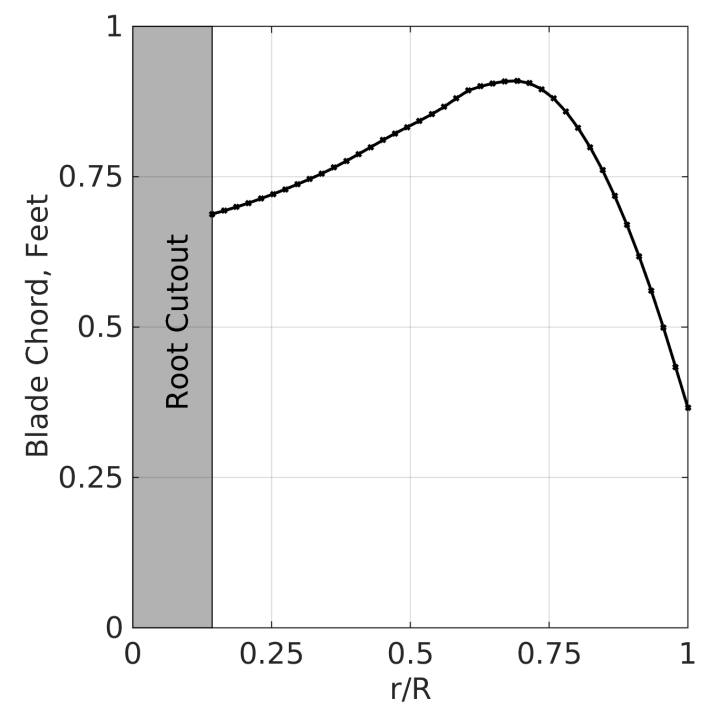

(b) Notional X2TD blade chord

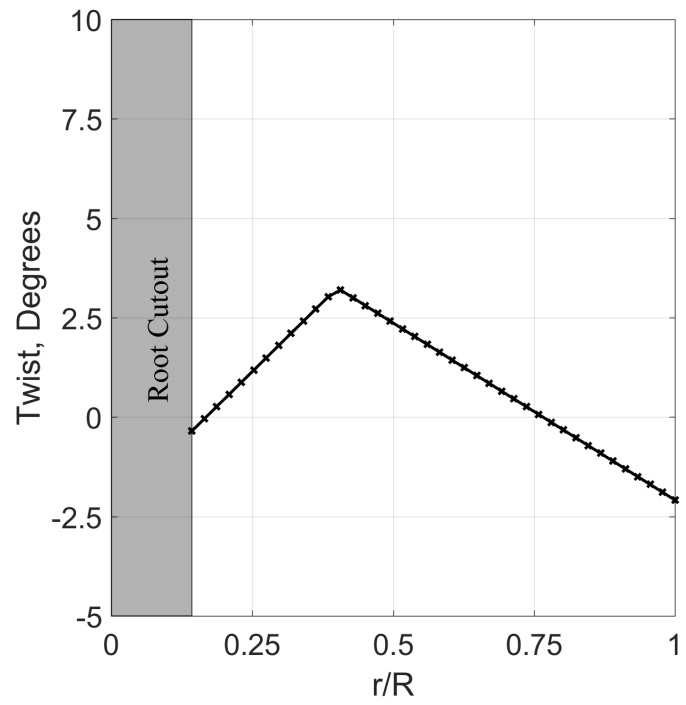

(c) Notional X2TD blade twist

Figure 2.4: Notional X2 rotor blade parameters. 
negative lift on the in-board sections of the retreating blade by aligning the inboard sections with the local incident velocity. The twist slope transitions from positive to a conventional negative slope near $40 \% R$.

In addition to the advanced blade design, main rotor RPM and LOS scheduling were implemented on the X2TD main rotor to improve the high-speed cruise efficiency. The main rotor RPM remained constant up to flight speeds of 200 knots. Beyond flight speeds of 200 knots the main rotor RPM was reduced such that $20 \%$ reduction was achieved at 280 knots and the advancing tip Mach number remained below 0.9. Figure 2.5(a) shows the rotor RPM ratio for a range of cruise speeds. The rotor RPM ratio data was digitized from Ref. [5]. The RPM of the main rotor is reduced with increase in advance ratio to reduce the compressibility effects on advancing blade tip.

Figure 2.5(b) shows the LOS data as a function of flight speed. The LOS data shown in Fig,2.5(b) in blue was digitized from X2TD flight tests discussed in detail in Ref. 6]. The high levels of scatter are present in the test data, which may be due to different flight conditions and regimes discussed in Ref. [6]. A linear line of fit was used to approximate the LOS data, which is consistent with how other researchers have treated this data [46]. The LOS scheduling is utilized to take advantage of the full potential of the advancing side of the rotor disk. The LOS is a measure of the displacement of thrust vector from the center of rotation. 


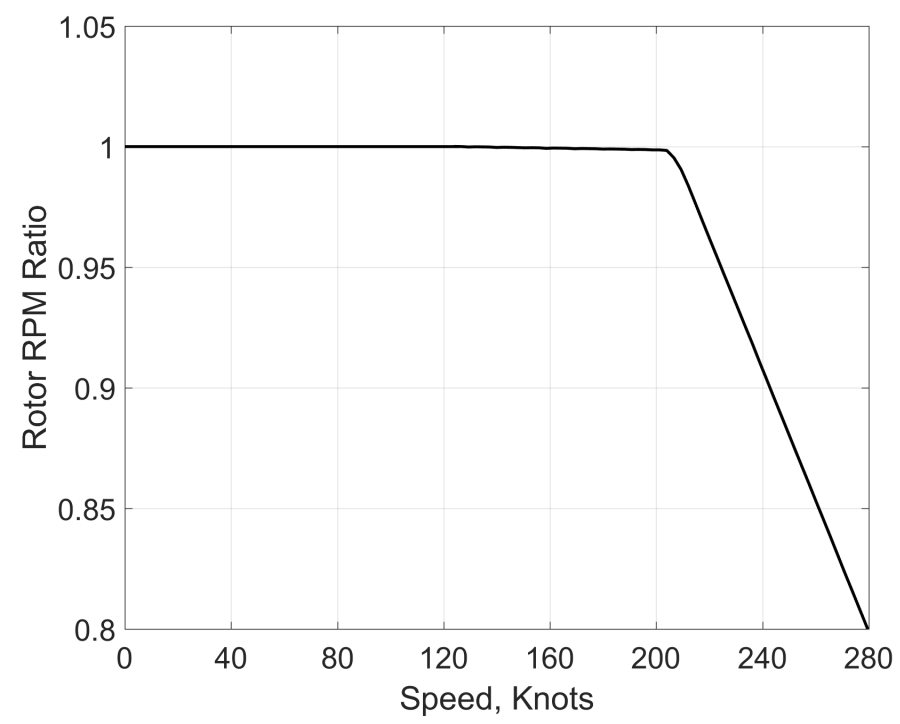

(a) Main rotor RPM ratio for various cruise speeds.

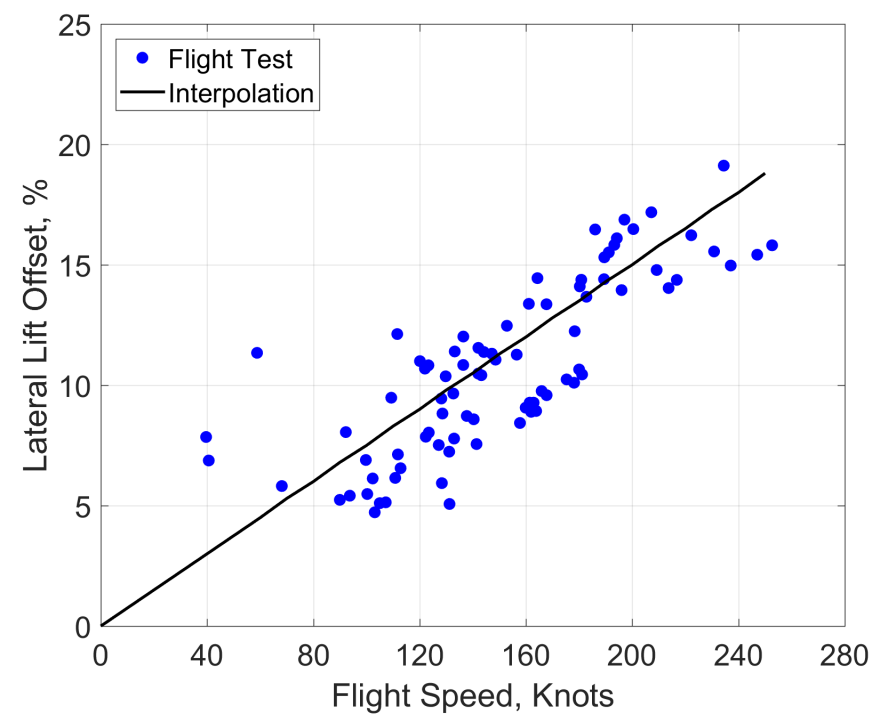

(b) Main rotor lateral LOS for various cruise speeds.

Figure 2.5: Main rotor RPM ratio for X2TD obtained from Ref. [5] and lateral LOS obtained from Ref. [6]. 


\subsection{Auxiliary Propulsor}

This section discusses the notional auxiliary propeller utilized in this work. A six bladed auxiliary propeller, with a radius of 3.33 feet, provides the propulsive force for the X2TD helicopter traveling at high cruise speeds. There is limited information available to the public regarding the geometry of the X2TD auxiliary propeller. Table 2.2 shows the propeller parameters given in Ref. [1] as well as assumed parameters. The propeller and the main rotor operate on a common drivetrain, therefore the RPM of the propeller is also reduced at high speeds, similarly to the main rotor. The required auxiliary propeller power, at 4000 feet density altitude, was digitized from Ref. [46] and is shown in Fig 2.6.

The photographs of the X2TD helicopter show the propeller to have high nonlinear twist and variable planform. However, due to lack of information regarding the exact blade geometry, various assumptions had to be made to model the propeller in the simulations. The notional pusher propulsor was modeled in this study as a six bladed propeller, with linear twist, constant chord and NACA0012 airfoil along the span. A linear twist for the notional X2TD propeller is shown in Fig,2.7. The propeller was designed with high twist to increase the angle of attack on the inboard stations of the blade. The angle of attack on the inboard stations of the propeller blade in high-speed cruise condition is reduced due to high inflow velocity. The radial chord distribution is shown in Fig 2.7(b). A simple constant chord distribution was chosen for the notional X2TD auxiliary propeller. The blades are treated as rigid without any elastic deformations. 


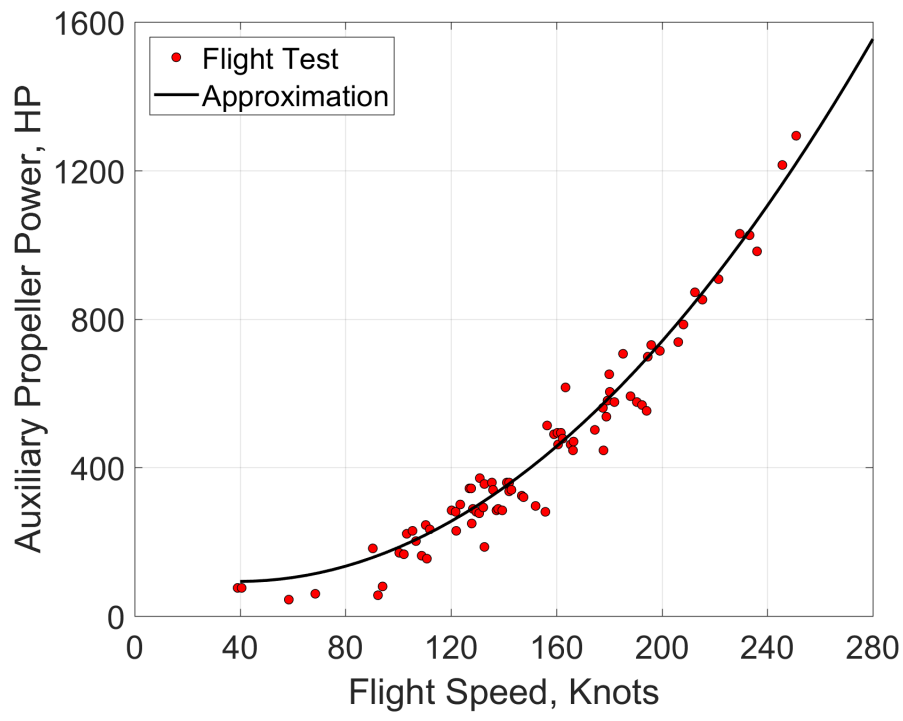

Figure 2.6: Auxiliary propeller power required at $4 \mathrm{~K}$ density altitude, obtained from flight tests [6].

Table 2.2: The notional X2TD auxiliary propeller parameters [1].

\begin{tabular}{l|c|c}
\hline \hline Parameter & Value & Units \\
\hline Number of blades & 6 & \\
Radius & 3.33 & $\mathrm{ft}$ \\
Root chord (notional) & 0.50 & $\mathrm{ft}$ \\
Root-cutout (notional) & 0.67 & $\mathrm{ft}$ \\
Rotation Speed - Hover to 200 knots & 2529 & $\mathrm{RPM}$ \\
Rotation Speed - 280 knots & 2041 & $\mathrm{RPM}$ \\
\hline \hline
\end{tabular}




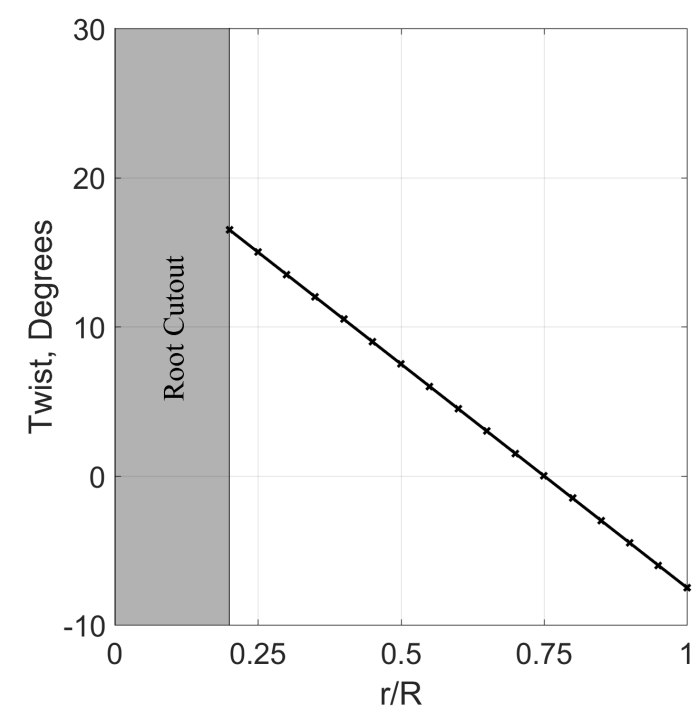

(a) Auxiliary propller twist distribution.

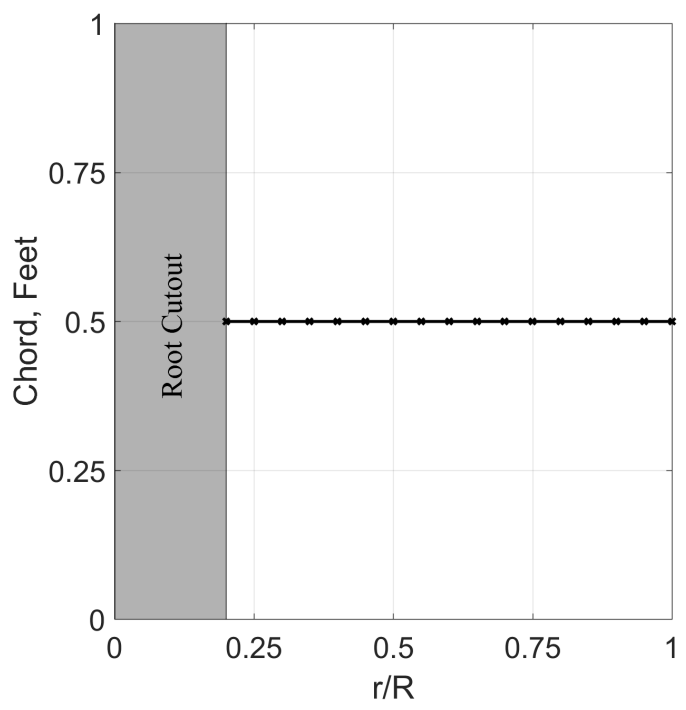

(b) Auxiliary propller chord distribution.

Figure 2.7: Notional X2TD auxiliary propeller twist and chord distribution.

\subsection{Airframe}

This section describes the airframe of the notional X2TD helicopter utilized in this study. The airframe of the notional X2TD helicopter resembled the actual 
Sikorsky X2TD, shown in Fig, with few exceptions. The notional airframe consists of a cigar shaped fuselage, empennage, mast and pylon, shown in Fig.2.8. All of the airframe components are very smooth, without any protrusions. The rotor hub fairings and rotor hubs not modeled in this study. The airframe CAD was assembled by Passe in [45].

The fuselage, shown in gray in Fig 2.8, is modeled as a smooth surface without any protrusions. The fuselage is 30.6 feet long. At it's maximum thickness, near the cockpit of the vehicle, the fuselage has a height of 4.9 feet. The flat plate drag area for the fuselage, given in Ref. [46], is 4.6 feet squared. The rotor shaft, shown in green in Fig, 2.8, is modeled as a cylindrical non-rotating mast with diameter of 0.9 feet. It extends from the top of the pylon to the upper rotor hub plane.

The empennage is composed of two horizontal stabilizers, shown in red in Fig 2.8, and a vertical tail, shown in blue. The horizontal tails of the notional X2TD resemble the horizontal tails on the actual X2TD helicopter during it's first flight shown in Fig. [6]. The equivalent flat plate surface area of the combined horizontal tails is 34 feed squared [37]. The actual aircraft included ground adjustable horizontal tail pitch incidence, as described in Ref. [1], however in this study the horizontal tail pitch incidence was fixed at +5 degrees with respect to the horizontal. The vertical tail also resembles that of the actual X2TD during early flight tests. An additional horizontal tail was installed near the bottom of the vertical tail following $12 t h$ flight test. However, the additional horizontal tail was not modeled in this study. The equivalent flat plate surface area of the vertical tail is 15.4 feet squared [37]. 


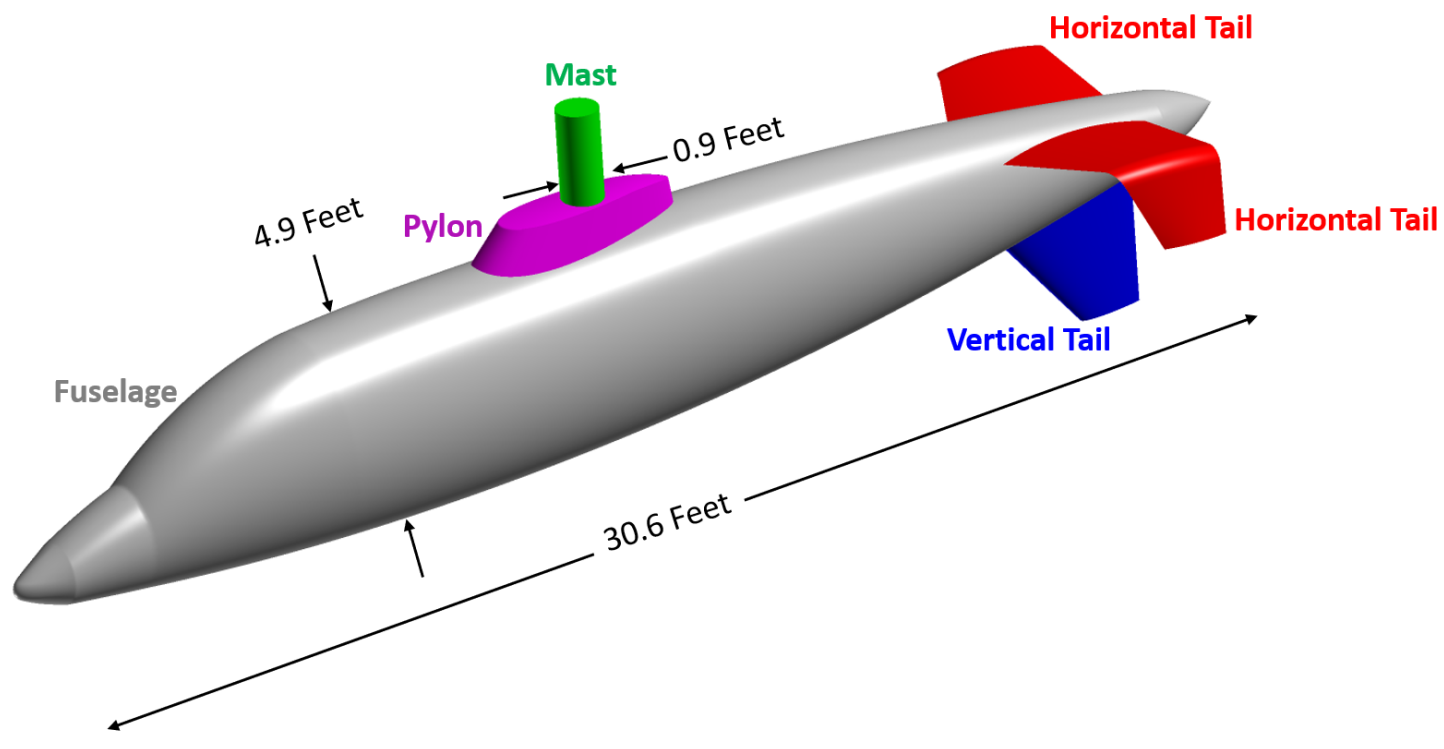

Figure 2.8: Notional X2TD airframe.

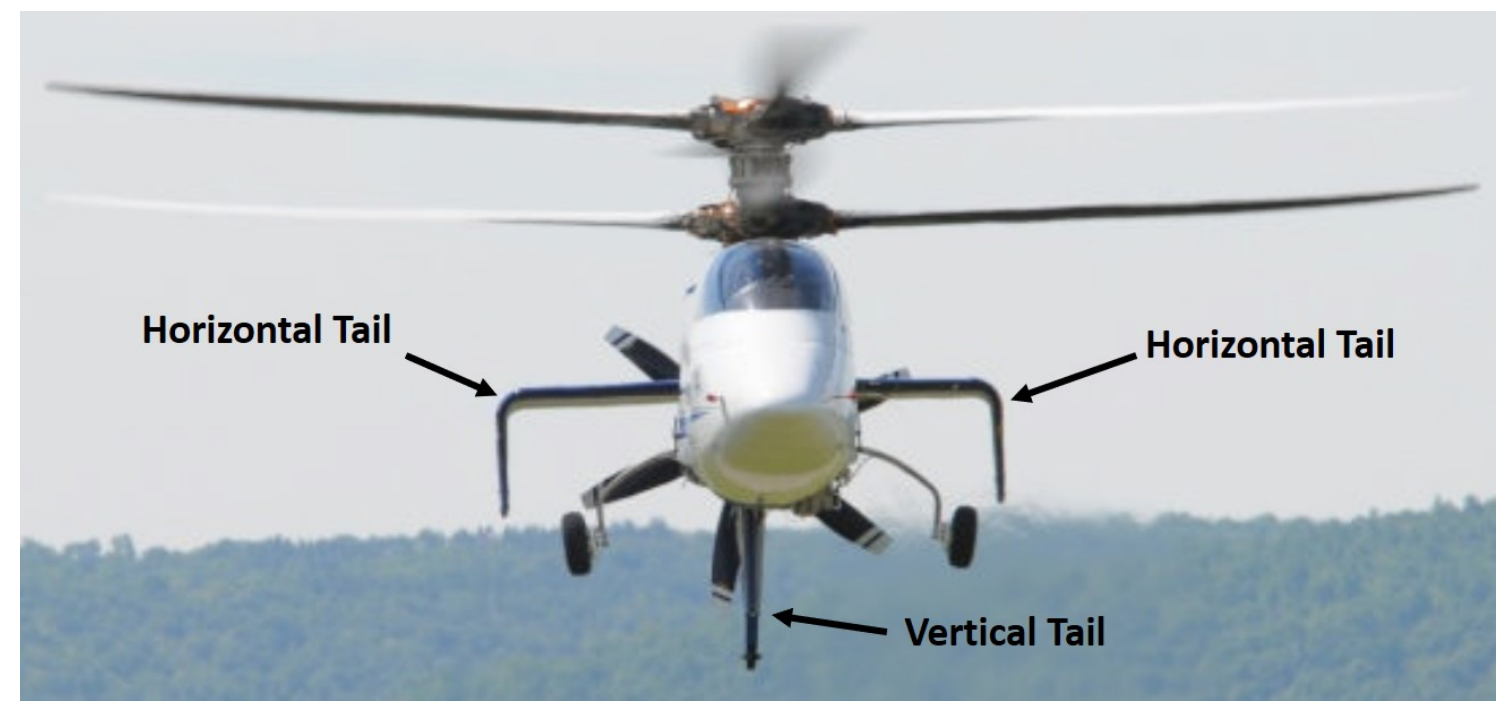

Figure 2.9: Sikorsky X2TD during it's first flight on August 27, 2008 [6]. 


\section{Comprehensive Analysis}

This chapter describes a comprehensive analysis (CA) methodology utilized in modeling the high-speed coaxial compound helicopter described in previous chapter. An in-house University of Maryland rotorcraft comprehensive analysis tool, PRASADUM, was utilized to perform trim and to model elastic blade deflections of the rotor blades. The in-house comprehensive analysis tool, PRASADUM, was developed by Sridharan, in Ref. 15, as part of a doctoral thesis. PRASADUM has been used for multiple CFD/CSD coupling studies by various researchers at University of Maryland, Ref.Ref. [38], 45], Ref. 447, as well as in the UH-60 Airloads workshop.

\subsection{Rigid Body Dynamics}

This section describes the rigid body dynamics of a coaxial compound helicopter modeled in PRASADUM. The Newton's second law is applied to the helicopter, modeled as a rigid body in space. The helicopter non-linear, rigid body equations of motion include force and moment equilibrium equations, shown in Eqs 3.1 - 3.3 and Eqs 3.4-3.6. A comprehensible, detailed derivation of the force and moment equations, as well as the specific variables, can be found in Ref. [48]. 
The right hand side of the equations represents the inertial loads acting on the helicopter at CG, which depends, directly and indirectly, on the nine rigid body states. The nine rigid body states are three linear and three angular body-axis velocities at $\mathrm{CG}$, as well as the three Euler angles that define the orientation of the helicopter with respect to the earth fixed axis. The body-axis coordinate system is shown in B. It is assumed that the center of gravity lies on the shaft, some distance below the rotor. The left-hand side of Eqs.3.1 - 3.3 and Eqs 3.4 - 3.6, accounts for the aerodynamic loads on the airframe (empennage and fuselage), rotor system as well as the propeller.

The force equilibrium equations in the body-axis frame:

$$
\begin{aligned}
X & =\mathrm{m}_{\mathrm{F}}\left(\dot{u}_{\mathrm{F}}+q_{\mathrm{F}} w_{\mathrm{F}}-r_{\mathrm{F}} v_{\mathrm{F}}+g \sin \theta_{\mathrm{F}}\right) \\
Y & =\mathrm{m}_{\mathrm{F}}\left(\dot{v}_{\mathrm{F}}+r_{\mathrm{F}} u_{\mathrm{F}}-p_{\mathrm{F}} w_{\mathrm{F}}-g \sin \phi_{\mathrm{F}} \cos \theta_{\mathrm{F}}\right) \\
Z & =\mathrm{m}_{\mathrm{F}}\left(\dot{w}_{\mathrm{F}}+p_{\mathrm{F}} v_{\mathrm{F}}-q_{\mathrm{F}} u_{\mathrm{F}}-g \cos \phi_{\mathrm{F}} \cos \theta_{\mathrm{F}}\right)
\end{aligned}
$$

The moment equilibrium equations in the body-axis frame:

$$
\begin{aligned}
& L=I_{x x} \dot{p}_{\mathrm{F}}-I_{x y}\left(\dot{q}_{\mathrm{F}}-p_{\mathrm{F}} r_{\mathrm{F}}\right)-I_{x z}\left(\dot{r}_{\mathrm{F}}+p_{\mathrm{F}} q_{\mathrm{F}}\right)-I_{y z}\left(q_{\mathrm{F}}^{2}-r_{\mathrm{F}}^{2}\right)-\left(I_{y y}-I_{z z}\right) q_{\mathrm{F}} r_{\mathrm{F}} \\
& M=I_{y y} \dot{q}_{\mathrm{F}}-I_{y z}\left(\dot{r}_{\mathrm{F}}-q_{\mathrm{F}} p_{\mathrm{F}}\right)-I_{y x}\left(\dot{p}_{\mathrm{F}}+q_{\mathrm{F}} r_{\mathrm{F}}\right)-I_{z x}\left(r_{\mathrm{F}}^{2}-p_{\mathrm{F}}^{2}\right)-\left(I_{z z}-I_{x x}\right) r_{\mathrm{F}} p_{\mathrm{F}} \\
& N=I_{z z} \dot{r}_{\mathrm{F}}-I_{z x}\left(\dot{p}_{\mathrm{F}}-r_{\mathrm{F}} q_{\mathrm{F}}\right)-I_{z y}\left(\dot{q}_{\mathrm{F}}+r_{\mathrm{F}} p_{\mathrm{F}}\right)-I_{x y}\left(p_{\mathrm{F}}^{2}-q_{\mathrm{F}}^{2}\right)-\left(I_{x x}-I_{y y}\right) p_{\mathrm{F}} q_{\mathrm{F}}
\end{aligned}
$$


The aerodynamic force and moment contributions from various components of a coaxial compound helicopter in the body-axis frame:

$$
\begin{aligned}
X & =X_{\mathrm{F}}+X_{\mathrm{HT}}+X_{\mathrm{VT}}+X_{\mathrm{PP}}+X_{\mathrm{UR}}+X_{\mathrm{LR}} \\
Y & =Y_{\mathrm{F}}+Y_{\mathrm{HT}}+Y_{\mathrm{VT}}+Y_{\mathrm{UR}}+Y_{\mathrm{LR}} \\
Z & =Z_{\mathrm{F}}+Z_{\mathrm{HT}}+Z_{\mathrm{VT}}+Z_{\mathrm{UR}}+Z_{\mathrm{LR}} \\
L & =L_{\mathrm{F}}+L_{\mathrm{HT}}+L_{\mathrm{VT}}+L_{\mathrm{UR}}+L_{\mathrm{LR}} \\
M & =M_{\mathrm{F}}+M_{\mathrm{HT}}+M_{\mathrm{VT}}+M_{\mathrm{UR}}+M_{\mathrm{LR}} \\
N & =N_{\mathrm{F}}+N_{\mathrm{HT}}+N_{\mathrm{VT}}+N_{\mathrm{UR}}+N_{\mathrm{LR}}
\end{aligned}
$$

The presence of the auxiliary propeller is accounted for in the $X$ force equation, which contains the contribution of the propulsive thrust from the auxiliary propeller. The thrust from the auxiliary propeller is assumed to act along the $X$ body axis, thus it does not impart any moments about the CG. The roll moment produced by the auxiliary propeller rotation is also neglected in this study, $\left(L_{P P}=0\right)$. Some additional assumptions, applicable to cases without airframe CFD/CSD coupling, are described below:

1. The fuselage normal force, side force, roll, pitch and yaw moments are zero, $\left(Y_{F}=0, Z_{F}=0, L_{F}=0, M_{F}=0, N_{F}=0\right)$.

2. The horizontal tail axial force, side force, roll and yaw moments are zero, $\left(X_{H T}=0, Y_{H T}=0, L_{H T}=0, N_{H T}=0\right)$.

If the full vehicle $\mathrm{CFD} / \mathrm{CSD}$ coupling procedure is performed, the above assumptions are relaxed and all of the CFD aerodynamic forces and moments are included as 
corrections to these equations.

\subsection{Aerodynamic Model}

This section describes the calculation of the aerodynamic forces and moments on the helicopter components needed to satisfy Eqs.3.1-3.3 and Eqs.3.4-3.6. The calculation of the airframe aerodynamic forces and moments is subdivided into three parts: fuselage, horizontal tail and vertical tail. In this study the flexibility of the airframe is neglected, the airframe is modeled as a rigid entity.

\subsubsection{Fuselage Airloads}

This subsection discusses the calculation of the aerodynamic forces on moments on the fuselage of the notional X2TD in the CA. The fuselage aerodynamic forces and moments are calculated from the fuselage aerodynamic coefficients given in the input lookup-tables. The first look-up table provides the lift, flat plate drag area and pitching moment as a function of angle of attack of the fuselage. The comprehensive analysis assumes that the flat plate drag area does not change with varying angles of attack. The fuselage look-up table assumes the fuselage lift and pitching moment are zero. In Ref. [46], Johnson reports the X2TD to have flat plate drag area of 4.6 feet squared. It was assumed that the flat plate drag area was defined using the maximum frontal area of the actual X2TD aircraft, it is unknown whether the flat plate drag area includes the frontal area of the rotor hubs and the mast pylon. 
Various CFD studies of the isolated airframe were conducted to verify that the flat plate drag area of the CFD model matched the number provided in Ref. [46]. The CFD model fuselage flat plate drag area was determined to be half of the reported one. It was assumed that the discrepancy was due to the missing hubs and the lack of protrusions in the CFD simulations. The second look-up table provides the fuselage side force, roll moment and yaw moment as functions of sideslip angle. All of the values in the second look-up table are assumed to be zero. Thus, the only force acting on the fuselage, in the $\mathrm{CA}$, is the drag force. The equation for the flat plate drag area is as follows:

$$
f=C_{D} S_{r e f}=\frac{D}{\frac{1}{2} \rho_{\infty} V_{\infty}}
$$

\subsubsection{Empennage Airloads}

This subsection describes the calculation of the horizontal tail and vertical tail airloads in the CA code. The horizontal and vertical tail aerodynamic forces and moments are calculated from the lift and drag coefficients provided in the input look-up tables. Both, horizontal and vertical tails are treated as low aspect ratio symmetric wings.

The lift coefficient in the look-up table, Eq3.7, was calculated from Prandtl's lifting-line theory, shown in Eq 3.8 , where $a_{0}$ is the airfoil lift curve slope, $A R$ is the aspect ratio, $\eta$ is the efficiency factor. The efficiency factor was assumed to be 0.85

for both horizontal and vertical tails. The airfoil curve slope of $2 \pi$ was assumed for 
both tails.

$$
\begin{array}{r}
C_{L}=C_{L \alpha} \alpha \\
C_{L_{\alpha}}=\frac{a_{o}}{1+\frac{a_{0}}{\pi A R \eta}}
\end{array}
$$

The tabulated drag coefficient in the look-up tables is the lift induced drag coefficient calculated using Eq 3.9 , where $C_{L}$ is the lift coefficient calculated from Prandtl's lifting line theory in Eq, 3.7 .

$$
C_{D_{i}}=\frac{C_{L}^{2}}{\eta \pi A R}
$$

The two horizontal tails are modeled as a single wing with aspect ratio equal to 3.5. The angle of attack which is used to determine the lift and drag coefficients from the horizontal tail look-up table is calculated based on the aircraft pitch attitude as well as the incidence angle of the horizontal tail. The vertical tail was modeled to have an aspect ratio of 1.2. The angle of attack used to determine lift and drag coefficients from the vertical tail look-up table is a function of the sideslip angle.

\subsubsection{Auxiliary Propeller Airloads}

This subsection explains the calculation of the notional X2TD auxiliary pro-

peller airloads in the CA. As previously shown in Eqs 3.1 - 3.3 and Eqs 3.4 - 3.6, only the propeller axial force is accounted for in the force and moment equations. It is assumed that the propeller thrust vector acts along the CG axis, therefore there is no pitching moment about the vehicle CG due to the propeller thrust. Also, the 
roll moment about the CG produced by the propeller is neglected. For the notional X2TD helicopter cases the auxiliary propeller thrust is tabulated in a speed schedule look-up table. The notional X2TD propeller thrust was calculated from the power curve shown in Fig 2.6 using a simplified expression from the momentum theory formulation for a propeller in climb, $P=T V$. In this expression $P$ is power, $T$ is thrust and $V$ is flight velocity. The power expression is purely induced. Later, the CFD simulations are used to correct the simplified expression.

\subsubsection{Coaxial Rotor System Airloads}

This section discusses the procedure used to calculate the rotor airloads needed for Eqs.3.1-3.3 and Eqs 3.4-3.6. The rotor airloads in these equations are the rotor forces in body frame and rotor moments about the $\mathrm{CG}$ of the vehicle. These forces and moments are calculated from the sectional rotor blade airloads. The rotor blade sectional airloads were calculated using a uniform inflow model, 2D steady airfoil tables and unsteady aerodynamics. The rotor blade properties discussed in the previous section are implemented in the CA model of the notional X2TD. The 2D airfoil look-up tables contain the steady lift, drag and pitching moment airfoil coefficients as a function of angle of attack and Mach number for airfoils shown in Fig 2.3(a). The data in the $2 \mathrm{D}$ airfoil look-up tables was compiled by Passe using a UMD unsteady RANS CFD code, TURNS [45]. For this study, the low angle of attack $C_{l}$ data, for all of the notional X2TD airfoils, was replaced with a linear $C_{l}$ curve slope of $2 \pi$, which resulted in an improved trim convergence. 
Only the steady component, $\lambda_{o}$, of the three state Peters-He dynamic inflow model, given in Eq.3.10, was used to compute the induced velocity at the rotor disk [49]. This low order assumption implies that the inflow is independent of the radial and azimuthal location. Since airloads were later corrected with high-fidelity CFD airloads, the use of uniform inflow was deemed sufficient. The detailed calculation of the dynamic inflow coefficients, including the $\lambda_{o}$, is provided in Ref. [49].

$$
\lambda(r, \psi)=\lambda_{o}+\lambda_{1 c} x \cos \psi+\lambda_{1 s} x \sin \psi
$$

The sectional lift and pitching moment, in the airfoil frame, were calculating using Eqs 3.11 and 3.12 , respectively [50]. The calculation of the lift and pitching moment can be partitioned into circulatory and non-circulatory components.

$$
\begin{aligned}
L & =L_{C}+L_{N C} \\
M & =M_{C}+M_{N C}
\end{aligned}
$$

The sectional circulatory and non-circulatory lift per unit span on the rotor blade are given by Eq $3.11[50]$. The circulatory lift, given in Eq.3.13, depends on the airfoil lift coefficient at a given rotor blade section [50]. The sectional lift coefficient at a given angle of attack and Mach number is obtained from a look-up table provided by the user as an input. The blade sectional angle of attack, $\alpha$, is obtained using Eq.3.14. The $U_{P}$ velocity includes the induced inflow velocity calculated using Eq 3.10 [50]. The magnitude of the free-stream velocity at the elastic axis was calculating using 
Eq.3.15 [50]. In this study, the elastic axis coincided with the quarter chord line on the rotor blade.

$$
\begin{array}{r}
L_{C}=1 / 2 \rho V_{\infty}^{2} c C_{l}(\alpha, M) \\
\alpha=\tan ^{-1} \frac{U_{P}}{\sqrt{U_{T}^{2}+U_{P}^{2}}} \\
V_{\infty}=\sqrt{U_{T}^{2}+U_{P}^{2}+U_{R}^{2}}
\end{array}
$$

The non-circulatory lift per unit span can be partitioned into two parts, shown in Eq. 3.16 , due to the pitching and plunging motion of the blade section. The first part is produced due to the plunging acceleration, $\ddot{h}$ of the blade section. The second part is produced due to the pitching of the blade section, $\alpha$.

$$
L_{N C}=\frac{\pi}{4} \rho c^{2} \ddot{h}+\frac{\pi}{4} \rho c^{2} V_{\infty} \dot{\alpha}
$$

The sectional pitching moment per unit span, about the elastic axis, can also be decomposed into the circulatory and non-circulatory pitching moments, as shown in Eq 3.12 [50]. The circulatory pitching moment is given by Eq.3.17, The first portion of the circulatory pitching moment depends on the sectional angle of attack and the pitching moment coefficient obtained from the airfoil look-up tables. The second portion of the circulatory pitching moment depends on the circulatory lift obtained from Eq 3.13 and the distance from the elastic axis to the aerodynamic center of the airfoil section. The third portion of the circulatory pitching moment depends on the time rate of change of the angle of attack. 


$$
M_{C}=1 / 2 \rho V_{\infty}^{2} c^{2} C_{m}(\alpha, M)+x_{a c} L_{C}+-\frac{\pi}{16} \dot{\alpha} \rho V_{\infty} c^{3}
$$

The non-circulatory pitching moment is given by 3.18 .

$$
M_{N C}=\frac{\pi}{4} \rho c^{2} \ddot{h} x_{m c}+\frac{\pi}{4} \rho c^{2} V_{\infty} \dot{\alpha} x_{0.75 c}
$$

The rotor blade sectional drag per unit span is a function of the sectional angle of attack and the Mach number. The airfoil drag coefficient is obtained from the look-up table discussed above.

$$
D=1 / 2 \rho V_{\infty}^{2} c(r) C_{d}(\alpha, M)
$$

The lift, drag and moment coefficients along the rotor blade are then used to calculate the sectional force and moment components in the rotating deformed airfoil frame. The sectional airloads are converted to the rotating undeformed airfoil frame. The rotor hubloads in the rotating frame are calculated by integrating the airloads along the span and finally, the rotating hubloads in the fixed frame are calculated. The hub forces can then be used in Eqs 3.1-3.3. The hub moments are transferred to the vehicle CG to satisfy Eqs $3.4-3.6$.

\subsection{Structural Model}

The rotor airloads described in previous subsection are highly dependent on the deformations of the rotor blade through $U_{P}$ and $U_{T}$. The blade deformations are influenced by the aerodynamic and inertial forces, as well as the blade structural 
properties and control inputs. Therefore, the problem is aeroelastically coupled and in order to obtain a representative airloads it is important to correctly model the deformation of the rotor blades.

The rotor blades modeled in the $\mathrm{CA}$ are geometrically exact representations of the notional X2TD rotor blades discussed in 2.1. The blade twist, chord and airfoil sections are specified in the input files. In this study, the main rotor blades are modeled as one dimensional isotropic Euler-Bernoulli beams, undergoing flap, lag and torsion. The Euler-Bernoulli assumption states that upon deformation, the plane cross-sections normal to the beam elastic axis remain plane and normal to the deformed elastic axis. The shear deformations of the beam are neglected. The translation of the elastic axis and the rotation of the cross-section can be used to calculate the deflection of any point on the cross-section.

The blade structural equations of motion are non-linear coupled partial differential equations. In order to obtain the structural deformations of the rotor blades, the structural equations of motion must be solved. The structural forces and moments are related to the external forces acting on the beam. A finite element discretization is used to eliminate the spatial coordinates and transform the PDEs into a system of non-linear coupled ordinary differential equations in time. Ten finite elements are used to discretized the blades in this study. Modal reduction was performed to reduce the number of equations, using rotating natural vibration modes. Six modes were used in this analysis. An in-depth development of the structural equations of motion and the solution methodology is presented by Sridharan in PRASADUM theory manual [50]. 


\subsection{Rotor Controls}

This section discusses the calculation of the main rotor pitch control angles. A pilot desired vehicle state results from the orientation of the rotor blades as they travel around the azimuth. The pilot provides the pitch control inputs which are transferred to the swashplate resulting in the re-orientation of the blades to a desired state. The pitch control angle is shown in Eq 3.20. For an articulated rotor, there is a phase delay between the pilot control inputs and the blade response, 80 to 90 degrees for hover. The phase delay, shown in Eq 3.21 , depends on the blade flap frequency and the Lock number. The pilot controls are: $\theta_{0}, \theta_{1 s}$ and $\theta_{1 c}$. The collective control angle, $\theta_{0}$, has an effect on the thrust and the yaw moment of the helicopter. For a conventional rotor with phase-offset of roughly 90 degrees, the longitudinal cyclic pitch, $\theta_{1 s}$, affects the pitching moment while the lateral cyclic pitch, $\theta_{1 c}$, controls the roll moment. For moderately stiff rotors, with high flap frequency, the phase delay is shorter, roughly 40 to 50 degrees.

$$
\begin{gathered}
\theta_{C}=\theta_{0}+\theta_{1 c} \cos (\psi)+\theta_{1 s} \sin (\psi) \\
\phi=\tan ^{-1} \frac{\gamma / 8}{\nu_{\beta}^{2}-1}
\end{gathered}
$$

The CA utilized for this study assumes that the notional X2TD rotor is moderately stiff, and neglects the phase delay between the pilot controls and the blade response. Therefore, the formulation assumes that the $\theta_{1 s}$ controls the roll moment while $\theta_{1 c}$ controls the rotor pitch moment. 
For a coaxial rotor system, upper and lower rotor have their own set of control angles. The collective angles for the upper and lower rotor, $\theta_{0}^{U}$ and $\theta_{0}^{L}$, are computed using Eqs 3.22 and 3.23 , where the $\theta_{0}$ is a mean collective and $\Delta \theta_{0}$ is the differential collective. The negative sign in Eqs 3.23 and 3.23 accounts for the direction of the rotation of the lower rotor.

$$
\begin{aligned}
& \theta_{0}^{U}=\theta_{0}+\Delta \theta_{0} \\
& \theta_{0}^{L}=\theta_{0}-\Delta \theta_{0}
\end{aligned}
$$

The cyclic pitch angles, $\theta_{1 c}^{U}$ and $\theta_{1 c}^{L}$, for the upper and lower rotor are computed using Eqs 3.24, The longitudinal lift-offset angle is assumed to be zero, the cyclic pitch angle $\theta_{1 c}$ is the same for both rotors.

$$
\theta_{1 c}^{U}=\theta_{1 c}^{L}=\theta_{1 c}
$$

The cyclic angles, $\theta_{1 s}^{U}$ and $\theta_{1 s}^{L}$, for the notional X2TD coaxial rotor were calculated using Eqs 3.25 and 3.26. As previously stated, the notional X2TD rotor was assumed to be moderately stiff and the phase delay between the control inputs and blade response was neglected. Therefore, the lateral lift-offset angle was included in the computation of the $\theta_{1 s}$ control because $\theta_{1 s}$ effects the roll moment when phase delay is zero. The swashplate phase offset angle is also neglected.

$$
\begin{aligned}
& \theta_{1 s}^{U}=\theta_{1 s}+\theta_{L O S} \\
& \theta_{1 s}^{L}=\theta_{1 s}-\theta_{L O S}
\end{aligned}
$$

The total pitch control angle for upper and lower rotor is given by Eqs 3.27 and 3.28. The azimuth, $\psi$, shown in Eqs 3.27 and 3.28 is the global azimuth angle, 
corresponding to a counter-clockwise rotation with zero over the tail of the vehicle. The blades cross-over the tail and every 90 degrees.

$$
\begin{aligned}
& \theta_{C}^{U}=\theta_{0}^{1}+\theta_{1 c} \cos (\psi)+\theta_{1 s}^{1} \sin (\psi) \\
& \theta_{C}^{L}=\theta_{0}^{2}+\theta_{1 c} \cos (\psi)+\theta_{1 s}^{2} \sin (\psi)
\end{aligned}
$$

\subsection{Trim}

The objective of this section is to describe the trim procedure and it's implementation in the in-house CA solver, PRASADUM. The goal of the trim procedure is to determine the trim state of a helicopter for a given flight condition. In this study, the vehicle is undergoing a steady, level flight. Trim can be defined as a balance of forces and moments on an aircraft. When the vehicle is trimmed, the main rotor blade dynamics are periodic and the inflow at the main rotor does not vary with consecutive revolutions. The concept of trim can be expressed as a coupled non-linear algebraic system of equations, given by Eq 3.29 51. The vector, $\boldsymbol{F}(\boldsymbol{x})$, represents a set of equations that must be satisfied for helicopter to be in trim. There exists a corresponding solution vector, $\boldsymbol{x}$, given in Eq3.31, which solves this algebraic system of equations for a given flight condition. The solution vector, $\boldsymbol{x}$, is unknown. Therefore, the objective of the trim procedure is to find vector $\boldsymbol{x}$ that solves the non-linear algebraic system of equations. Both, vectors, $\boldsymbol{x}$ and $\boldsymbol{F}(\boldsymbol{x})$, consist of the rigid body, inflow and main rotor components [51]. The rest of this section is dedicated to describing the components of vectors $\boldsymbol{F}$ and $\boldsymbol{x}$. 


$$
\begin{gathered}
\boldsymbol{F}(\boldsymbol{x})=0 \\
\boldsymbol{F}=\left[\begin{array}{lll}
\boldsymbol{F}_{B} & \boldsymbol{F}_{I} & \boldsymbol{F}_{R}
\end{array}\right]^{T} \\
\boldsymbol{x}=\left[\begin{array}{lll}
\boldsymbol{x}_{B} & \boldsymbol{x}_{I} & \boldsymbol{x}_{R}
\end{array}\right]^{T}
\end{gathered}
$$

Vector, $\boldsymbol{F}_{B}$, represents the rigid body dynamic equations of the vehicle, shown in Eqs 3.1 through 3.6. The definition of trim implies that the vehicle translation and angular accelerations, along the body axes, are zero. The translation accelerations $(\dot{u}, \dot{v}, \dot{w})$ and angular accelerations $(\dot{p}, \dot{q}, \dot{r})$ can be calculated from Eqs 3.1 through 3.6. Extending this definition to rotorcraft application requires that the summation of forces and moments over one rotor revolution is equal to zero, which can be shown with an equivalent condition in Eqs. 3.32 through 3.37 Ref. [50].

$$
\begin{aligned}
& \int_{0}^{T} \dot{u}_{\mathrm{F}} d t=\epsilon_{\mathrm{RB} 1}=0 \\
& \int_{0}^{T} \dot{v}_{\mathrm{F}} d t=\epsilon_{\mathrm{RB} 2}=0 \\
& \int_{0}^{T} \dot{w}_{\mathrm{F}} d t=\epsilon_{\mathrm{RB} 3}=0 \\
& \int_{0}^{T} \dot{p}_{\mathrm{F}} d t=\epsilon_{\mathrm{RB} 4}=0 \\
& \int_{0}^{T} \dot{q}_{\mathrm{F}} d t=\epsilon_{\mathrm{RB} 5}=0 \\
& \int_{0}^{T} \dot{r}_{\mathrm{F}} d t=\epsilon_{\mathrm{RB} 6}=0
\end{aligned}
$$


The vector $\boldsymbol{x}_{B}$, representing the rigid body unknowns, is given in Eq.3.38. The vector of the rigid body unknowns consists of trim control angles $\left(\theta_{0}, \theta_{1 c}, \theta_{1 s}\right.$ and $\left.\Delta \theta_{0}\right)$, fuselage angle of attack $\left(\alpha_{F}\right)$ and the lift-offset angle $\left(\theta_{L O S}\right)$.

$$
\boldsymbol{x}_{B}=\left[\begin{array}{llllll}
\theta_{0} & \theta_{1 c} & \theta_{1 s} & \delta \theta_{0} & \alpha_{F} & \theta_{L O S}
\end{array}\right]^{T}
$$

Equation 3.32 is an equivalent representation of the force equilibrium in the $\mathrm{X}$ direction, given by Eq,3.1. The unknown trim variable from vector $\boldsymbol{x}_{B}$, corresponding to Eq.3.32 is $\alpha_{F}$. The propulsive force produces by the auxiliary propeller is assigned by the user in a look-up table, based on the speed of the vehicle and the power given in Ref. [46]. The propulsive force, is counteracted by the vehicle $\mathrm{H}$ force. Therefore, the angle of attack of the vehicle, $\alpha_{F}$, must be solved for in order to counteract the propeller propulsive force resulting in the vehicle pitch attitude being an unknown trim control for this equation.

The trim parameters, $\theta_{1 s}$ and $\theta_{L O S}$, shown in Eq.3.38, affect the side force represented by Eq.3.33 and roll moment represented by Eq.3.35. The LOS angle depends on the LOS requirement identified by the user in the inputs. The LOS requirement is specified in a lookup-table and was obtained from linear interpolation of the available test data given for the X2TD rotor in Ref. [5]. During the propulsive trim, the Eq.3.39, must satisfy the LOS given in the lookup-table. The $\theta_{L O S}$ is calculated based on the given LOS value.

$$
L O S_{r e q}=\frac{\Delta M_{x}}{T R}
$$


The trim parameters, $\theta_{0}$ and $\delta \theta_{0}$, govern the vertical force equation, Eq 3.34 , and the yaw moment equation Eq3.37. Equation 3.36 is an equivalent representation of the pitching moment equilibrium about the vehicle CG. For the notional X2TD the horizontal tails produce significant pitch down moment about the vehicle CG. The main rotor counteracts the pitch-down moment produced by the horizontal tails. The trim parameter for this equation is the cyclic pitch angle, $\theta_{1 c}$.

The inflow trim equations are given by Eqns 3.413 .43 [51]. These equations enforce the conditions that the inflow does not change between consecutive iterations. The inflow is described by Eqn 3.10 . The main rotor inflow unknowns, $\boldsymbol{x}_{I}$, are given by Eq 3.44. However, components $\lambda_{1 c}$ and $\lambda_{1 s}$ of Eq 3.44 are neglected for this study because only the uniform inflow component was considered.

$$
\begin{aligned}
& \int_{0}^{T} \dot{\lambda}_{0} d t=0 \\
& \int_{0}^{T} \dot{\lambda}_{1 c} d t=0 \\
& \int_{0}^{T} \dot{\lambda}_{1 s} d t=0
\end{aligned}
$$

$$
\boldsymbol{x}_{I}=\left[\begin{array}{lll}
\lambda_{o} & \lambda_{1 c} & \lambda_{1 s}
\end{array}\right]^{T}=\left[\lambda_{0}\right]^{T}
$$

The ordinary differential equations of motions governing the structural dynamics of a single rotor blade, expressed in generalized coordinates, are given by 
Eq.3.45 [51]. The helicopter rotor blade response and blade deformations are periodic if the helicopter is in a trim condition. Therefore, the blade motions can be approximated using Fourier Series truncation given in Eq.3.46 [51].

$$
\begin{gathered}
\ddot{\boldsymbol{q}}=\boldsymbol{f}_{\boldsymbol{q}}(\boldsymbol{q}, \dot{\boldsymbol{q}}) \\
q^{k}(\psi) \approx q_{a p p x}^{k}(\psi)=q_{0}^{k}+\sum_{j=1}^{N_{h}}\left(q_{j c}^{k} \cos j \psi+q_{j s}^{k} \sin j \psi\right)
\end{gathered}
$$

In Eq $3.46, q^{k}(\psi)$ represents the generalized coordinates of $k^{\text {th }}$ blade modes. Because the $\boldsymbol{x}$ is no longer exact but approximate, there will exist a residual such as shown in Eq. 3.47 [51].

$$
\epsilon(\psi)=\ddot{\boldsymbol{q}}_{\text {appx }}-\boldsymbol{f}_{\boldsymbol{q}}\left(\boldsymbol{q}_{\text {appx }}, \dot{\boldsymbol{q}}_{\text {appx }}\right)
$$

In order for the residual, shown in Eq.3.47, to be minimized, Eqs 3.48 through 3.50 must be enforced if the Galerkin method is used [51]. The generalized coordinates of the $N_{m}$ blades modes that satisfy Eqs 3.48 through 3.50 are given in $\boldsymbol{x}_{R}$ in Eq.3.51.

$$
\begin{aligned}
& \int_{0}^{2 \pi} \epsilon^{k}(\psi) d \psi=0 \\
& \int_{0}^{2 \pi} \epsilon^{k}(\psi) \cos (j \psi) d \psi=0 \\
& \int_{0}^{2 \pi} \epsilon^{k}(\psi) \sin (j \psi) d \psi=0 \\
& \boldsymbol{x}_{R}=\left[\begin{array}{lllllllllll}
q_{0}^{1} & q_{1 c}^{1} & q_{1 s}^{1} & q_{2 c}^{1} & q_{2 s}^{1} \ldots & q_{N_{h} c}^{1} & q_{N_{h} s}^{1} \ldots q_{0}^{N m} & q_{1 c}^{N_{m}} & q_{1 s}^{N_{m}} \ldots & q_{N_{h} c}^{N_{m}} & q_{N_{h} s}^{N_{m}}
\end{array}\right]^{T}
\end{aligned}
$$




\subsection{Trim Solution Method}

This section will describe the method utilized to solve the system of algebraic equations given in Eq 3.29, A non-linear equation solver, HYBRD1 from the MINPACK-1 library, Ref. [52], solves this system of non-linear equations in PRASADUM.

$$
f_{i}=\left(x_{1}, x_{2}, \ldots, x_{n}\right)=0, \quad 1 \leqslant i \leqslant n
$$

In the context of this work, the values $x_{1}, x_{2}, \ldots, x_{n}$ represent the trim parameters, discussed in previous section, which include rotor control angles, vehicle attitude angles, inflow constants and blade modes. In the coaxial helicopter propulsive trim, lift-offset and propeller thrust are also trim variables. Trim enforces that the user specified lift-offset and the propeller thrust are satisfied.

The functions, $f_{1}, f_{2}, \ldots, f_{n}$, relate the trim parameters to the force and moment equilibrium of the vehicle, the rotor inflow states, and beam deformations. The user provides an initial approximations $\boldsymbol{x}$ to the solution of the functions $f_{1}, f_{2}, \ldots, f_{n}$. Usually, the initial guess does not solve the system of equations, there exists a residual. The solution algorithm determines a correction to the initial guess which minimizes the residual. The updated guess is then used as a new starting point to evaluate the functions $f_{1}, f_{2}, \ldots, f_{n}$. 


\subsection{Validation of the Notional X2TD Comprehensive Analysis Model}

The in-house comprehensive solver, PRASADUM, utilized for this study has been widely used within the UMD rotorcraft community [45], 444, [38, [47, [53], [54], 55]. Thus, the validation focused on verifying the notional X2TD model, as well as the main rotor structural properties, implemented in the CA instead of the implementation of the CA.

The notional X2TD power obtained with the CA was compared with the rotor power given in Ref. [6]. Figure 3.1 compares the rotor power from flight data and the CA, as well as the data fit line for the flight data. The CA utilized 6 blade modes and 4 harmonics, as well as a free-wake methodology to for the validation case [56]. It can be observed that the predicted power matches well with the digitized power from Ref. [6].

Figure 3.2 shows the notional X2TD fanplot. A notional X2TD fanplot, calculated using the in-house CA, is compared with the digitized data from the Sikorsky X2TD fanplot obtained from Ref. [1]. The notional X2TD main rotor blade mass and stiffness properties in the CA, were parametrically derived and scaled from the Sikorsky XH-59A main rotor blades by Passe in Ref. 45] to closely match the fanplot data from Ref. [1]. Passe also compared the derived frequencies to those obtained by Johnson using CAMRAD II [10] in Ref. [46].

There is good agreement between the first flap, first chordwise, second flap and first torsional frequencies predicted using CA and those given in Ref. [1]. However, there is a large disparity between the notional X2TD and the actual X2TD third 


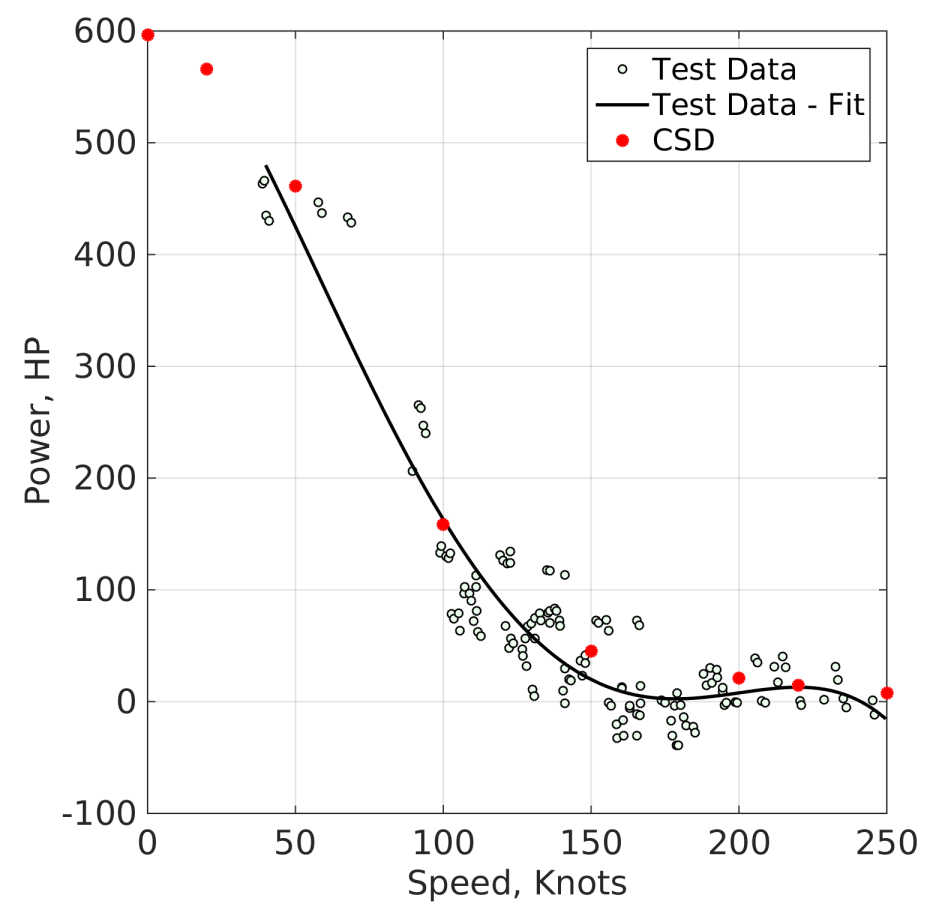

Figure 3.1: The power validation for the notional X2TD.

flapwise and the second chordwise frequencies, Ref. [1]. Nevertheless, the third flapwise and second chordwise frequencies match well with those obtained by Johnson in Ref. [46] and were deemed sufficient for this study. 


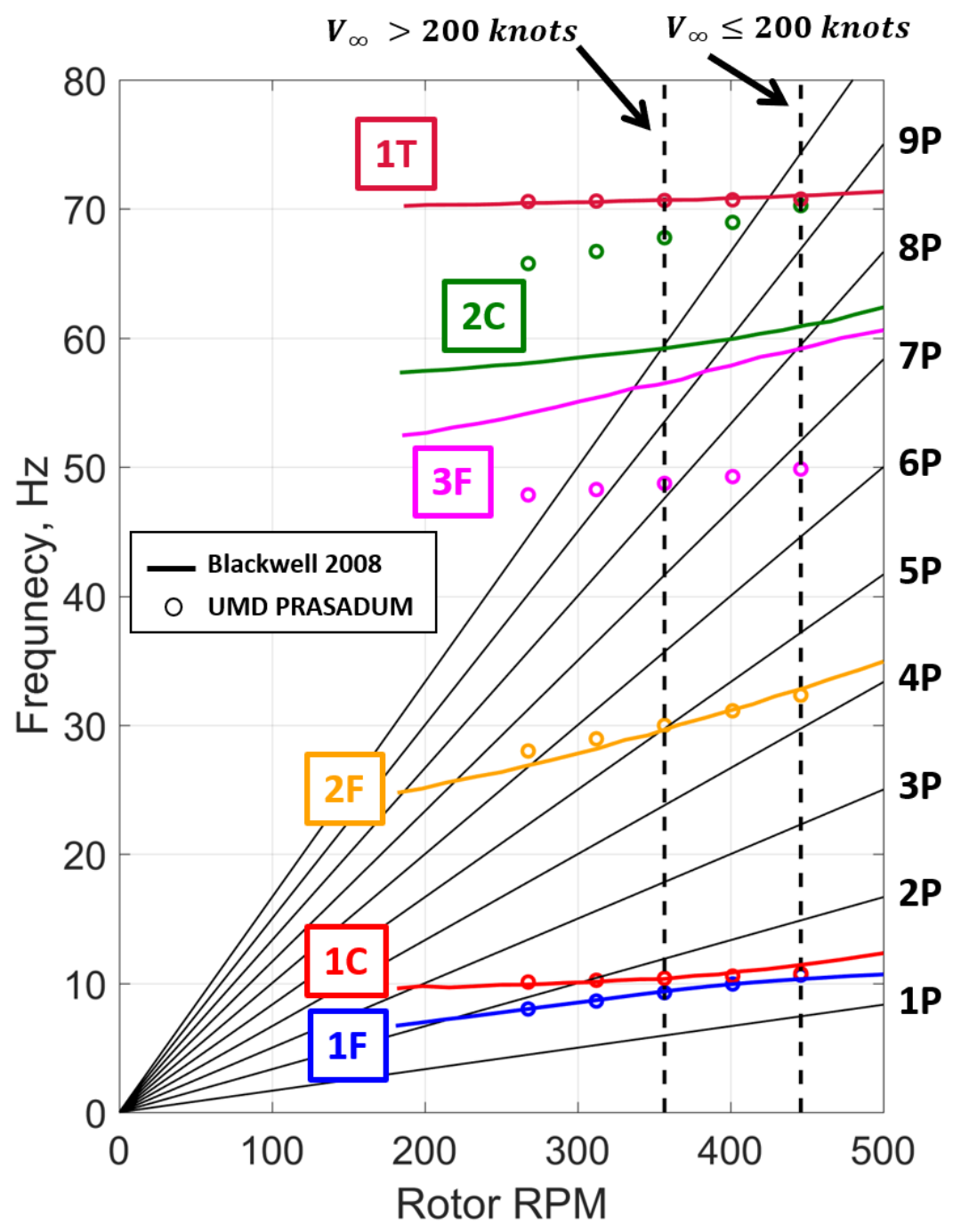

Figure 3.2: The fanplot validation for the notional X2TD. 


\section{Computational Fluid Dynamics}

This chapter discusses the fundamental equations of fluid motion and the computational approach used to solve these equations. The fundamental equations of the fluid motion, that are solved in the computational codes used for this study, are the three dimensional, unsteady, compressible Navier-Stokes equations. The Navier-Stokes equations are mathematical laws that enforce the conservation of mass, momentum and energy. The two near-body solvers which were chosen for this study are OVERFLOW and FUN3D, as part of the CREATE-AV Helios framework. The off-body is solved using a Cartesian flow solver, SAMCart. The objective of the CFD in this work is to solve for flow variables and obtain high-fidelity aerodynamic loads on the helicopter components.

\subsection{Governing Equations}

This section discusses the three dimensional, unsteady Navier-Stokes equations utilized for this study. The three-dimensional Navier-Stokes equations may be written in a strong conservation form, shown in Eq. 4.1 [57].

$$
\frac{\partial \boldsymbol{Q}}{\partial t}+\frac{\partial \boldsymbol{E}_{\boldsymbol{c}}}{\partial x}+\frac{\partial \boldsymbol{F}_{\boldsymbol{c}}}{\partial y}+\frac{\partial \boldsymbol{G}_{\boldsymbol{c}}}{\partial z}=\frac{\partial \boldsymbol{E}_{\boldsymbol{v}}}{\partial x}+\frac{\partial \boldsymbol{F}_{\boldsymbol{v}}}{\partial y}+\frac{\partial \boldsymbol{G}_{\boldsymbol{v}}}{\partial z}
$$


The vector $\boldsymbol{Q}$ is a vector of conserved variables given by Eq4.2, where the variables $\rho, u, v, w$ and $e$ denote the local flow density, the three velocity components and the total energy, respectively.

$$
\boldsymbol{Q}=\left[\begin{array}{c}
\rho \\
\rho u \\
\rho v \\
\rho w \\
e
\end{array}\right]
$$

The vectors $\boldsymbol{E}_{\boldsymbol{c}}, \boldsymbol{F}_{\boldsymbol{c}}$ and $\boldsymbol{G}_{\boldsymbol{c}}$, given in Eq. 4.3, are the inviscid fluxes in the $x, y, z$ directions.

$$
\boldsymbol{E}_{\boldsymbol{c}}=\left[\begin{array}{c}
\rho u \\
\rho u^{2}+p \\
\rho u v \\
\rho u w \\
u(e+p)
\end{array}\right] \quad \boldsymbol{F}_{\boldsymbol{c}}=\left[\begin{array}{c}
\rho v \\
\rho u v \\
\rho v^{2}+p \\
\rho v w \\
v(e+p)
\end{array}\right] \quad \boldsymbol{G}_{\boldsymbol{c}}=\left[\begin{array}{c}
\rho w \\
\rho u w \\
\rho v w \\
\rho w^{2}+p \\
w(e+p)
\end{array}\right]
$$

The pressure, denoted by $p$, in the Eq4.3 can be obtained by using the equation of state applied to calorically perfect gas, Eq4.4. The ratio of specific heats, $\gamma$, was assumed to be 1.4 .

$$
p=(\gamma-1)\left[e-\frac{1}{2} \rho\left(u^{2}+v^{2}+w^{2}\right)\right]
$$


The vectors $\boldsymbol{E}_{v}, \boldsymbol{F}_{v}$ and $\boldsymbol{G}_{v}$, given in Eq 4.5, are the viscous fluxes in the $x, y, z$ directions.

$$
\boldsymbol{E}_{\boldsymbol{v}}=\left[\begin{array}{c}
0 \\
\tau_{x x} \\
\tau_{y x} \\
\tau_{z x} \\
E_{v 5}
\end{array}\right] \boldsymbol{F}_{\boldsymbol{v}}=\left[\begin{array}{c}
0 \\
\tau_{x y} \\
\tau_{y y} \\
\tau_{z y} \\
F_{v 5}
\end{array}\right] \boldsymbol{G}_{\boldsymbol{v}}=\left[\begin{array}{c}
0 \\
\tau_{x z} \\
\tau_{y z} \\
\tau_{z z} \\
G_{v 5}
\end{array}\right]
$$

where

$$
\begin{aligned}
& E_{v 5}=u \tau_{x x}+v \tau_{x y}+w \tau_{x z}-q_{x} \\
& F_{v 5}=u \tau_{y x}+v \tau_{y y}+w \tau_{y z}-q_{y} \\
& G_{v 5}=u \tau_{z x}+v \tau_{z y}+w \tau_{z z}-q_{z}
\end{aligned}
$$

The stress tensor, denoted by $\boldsymbol{\tau}$, is obtained using the Stokes' hypothesis, assuming Newtonian fluid [58]. The $\mu$ and $\lambda=-2 \mu / 3$ are the first and second coefficients of viscosity. The Sutherland's formula, Eq4.10, can be used to calculate the first coefficient of viscosity, $\mu$, where $C_{1}$ and $C_{2}$ are constants for air. The temperature, $T$, can be calculated using the perfect gas law, Eq.4.11. The Kronecker delta function is denoted by $\delta_{i j}$, shown in Eq4.12.

$$
\begin{gathered}
\tau_{i j}=\mu\left(\frac{\partial u_{i}}{\partial x_{j}}+\frac{\partial u_{j}}{\partial x_{i}}\right)+\lambda\left(\frac{\partial u_{i}}{\partial x_{j}}\right) \delta_{i j} \\
\mu=\frac{C_{1} T^{(3 / 2)}}{T+C_{2}} \\
T=\frac{P}{\rho R}
\end{gathered}
$$




$$
\delta_{i j}= \begin{cases}0, & i \neq j \\ 1, & i=j\end{cases}
$$

The $q_{x}, q_{y}$ and $q_{z}$ components of the vector $\boldsymbol{q}$ present in Eqs 4.64 4.8, represent the rates of thermal conduction. The rates of thermal conduction, obtained using the Fourier's Law of heat conduction shown in Eq.4.13, are dependent on the temperature and the thermal conductivity of the fluid Ref. [59].

$$
q_{j}=k \frac{\partial d T}{\partial x_{j}} \quad j=x, y, z
$$

\subsection{Reynolds Averaged Navier-Stokes Equations}

The flow field surrounding a helicopter is inherently turbulent, containing a wide range of length and time scales. An extremely fine grid and a very small time step is required to properly resolve the smallest length and time scales in the flow field, making the solution process infeasible for practical applications. A workaround involves solving only for the mean flow quantities while modeling turbulent fluctuations, which can be done using the Reynolds Averaged Navier-Stokes (RANS) Equations .

The instantaneous flow variables $\left(u_{i}, \rho, p, T\right.$,), denoted by $\phi$ in Eq4.14, can

be decomposed into the mean, $\bar{\phi}$, and fluctuating, $\phi^{\prime}$, quantities using Reynolds decomposition [60].

$$
\phi=\bar{\phi}+\phi^{\prime}
$$


The Reynolds decomposition of the flow variables is substituted into the instantaneous governing equations shown in Eq4.1. The modified equations are then averaged resulting in cancellation and simplification of numerous terms based on the mathematical identities for averaged and fluctuating quantities. An in-depth derivation of the RANS equations can be found in Ref. [60] and Ref. [61]. The Reynolds Stress Tensor, given in Eq4.15, is one of the key differences between the original governing equations and the averaged governing equations. The Reynolds Stress Tensor depends on the turbulent fluctuating quantities, $u_{i}^{\prime}$ and $u_{j}^{\prime}$.

$$
\left(\bar{\tau}_{i j}\right)_{t u r b}=-\rho \overline{u_{i}^{\prime} u_{j}^{\prime}}
$$

The Boussinesq eddy viscosity hypothesis, given in Eq.4.16, can be used to obtain the Reynolds Stress Tensor [62]. A turbulence closure model is then used to determine the turbulent viscosity, $\mu_{t u r b}$. In this study, Spalart-Allmaras (SA), a one equation turbulence model is utilized [63]. Spalart-Allmaras has been validated and extensively used for practical helicopter simulations.

$$
\left(\bar{\tau}_{i j}\right)_{t u r b}=\mu_{t u r b}\left(\frac{\partial u_{i}}{\partial x_{j}}+\frac{\partial u_{j}}{\partial x_{i}}+\frac{2}{3} \frac{\partial u_{i}}{\partial x_{j}} \delta_{i j}\right)
$$

\subsection{CFD Solvers}

This section describes the CFD framework utilized for this study. A CFD framework, CREATE ${ }^{T M}$-AV Helios (version 9.1), was used to perform the CFD simulations [64]. The CREATE ${ }^{T M}$-AV Helios has developed over the years into a so- 
phisticated rotorcraft analysis tool which utilizes multi-mesh, multi-solver paradigm to perform high-fidelity rotorcraft flow simulations [65], [66], [67]. The overset domain connectivity within Helios, between the near-body and off-body solvers, is performed by the PUNDIT software [68]. A Mesh Motion Loading and Displacement Interface (Melodi), within the Helios framework, oversees the fluid structure interaction coupling, including the application of the prescribed elastic blade deflections to the blade mesh [69].

Various near-body solvers were used for the study, including OVERFLOW and FUN3D. Figure 4.1 shows an overview of the mesh system and the dedicated solvers, as well as the number of grid points in each mesh body. The CFD simulations were run in a fully turbulent mode using the Spalart-Allmaras turbulence model for
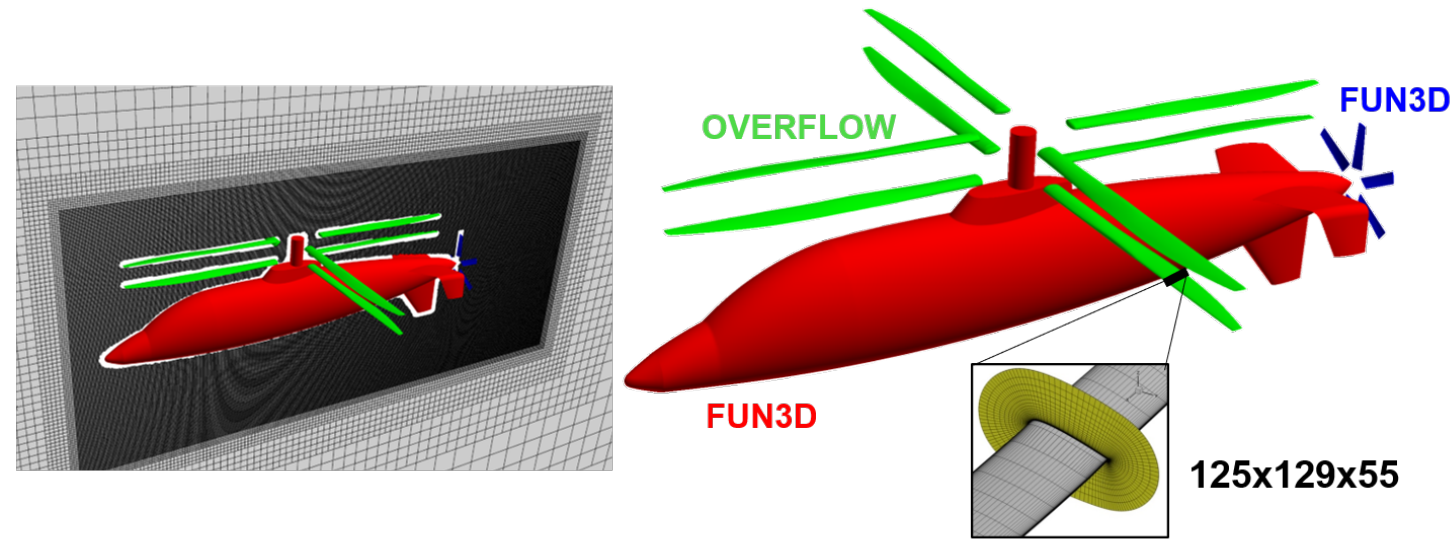

\begin{tabular}{|c|c|c|c|}
\cline { 2 - 4 } \multicolumn{1}{c|}{} & Mesh Type & Number of Grid Points & Solver \\
\hline Coaxial rotor system & Structured (O-O) & $7.1 \mathrm{M}(0.88 \mathrm{M}$ per blade $)$ & OVERFLOW \\
Fuselage & Unstructured & $15.6 \mathrm{M}$ & FUN3D \\
Auxiliary Propulsor & Unstructured & $13.2 \mathrm{M}(2.2 \mathrm{M}$ per blade $)$ & FUN3D \\
Off-body & Cartesian & $115 \mathrm{M}$ & SAMCart \\
\hline
\end{tabular}

Figure 4.1: Helicopter mesh system information. 
OVERFLOW and FUN3D near-body solvers, as well as for the off-body SAMCart solver. The simulations were run using a main rotor quarter degree time step.

\subsubsection{Coaxial Main Rotor - OVERFLOW}

The structured CFD solver, OVERFLOW (version 2.2n) developed at NASA, was used for the main rotor blades [70]. OVERFLOW was initially developed as a finite difference CFD solver which over time evolved to include finite volume capabilities. The OVERFLOW code solves the unsteady RANS equations. The formulation for the unsteady RANS equations is discussed in the previous section. Numerous spatial and temporal discretization schemes are available in OVERFLOW.

A fourth order central differencing scheme with a stabilizing fifth order artificial dissipation was used for the discretization of the inviscid fluxes in this study [71]. The usage of central differencing scheme is common for rotorcraft applications [39], [40], [72], [73], . The Roe flux splitting scheme [74] was also tested for this study but the convergence was slower than that of the central scheme, for the same number of sub-iterations. This may be attributed to the stiffness of the problem due to the low Mach number flow near the reverse flow region. A second order central differencing was used for the viscous fluxes. The second order backwards differencing formula (BDF2) was utilized for the temporal discretization [75], [76].

The initial studies were performed using 20 Newton sub-iteration but later increased to 40 to improve the convergence at high forward flight speeds.

The structured near-body notional X2TD blade meshes utilized in this study 


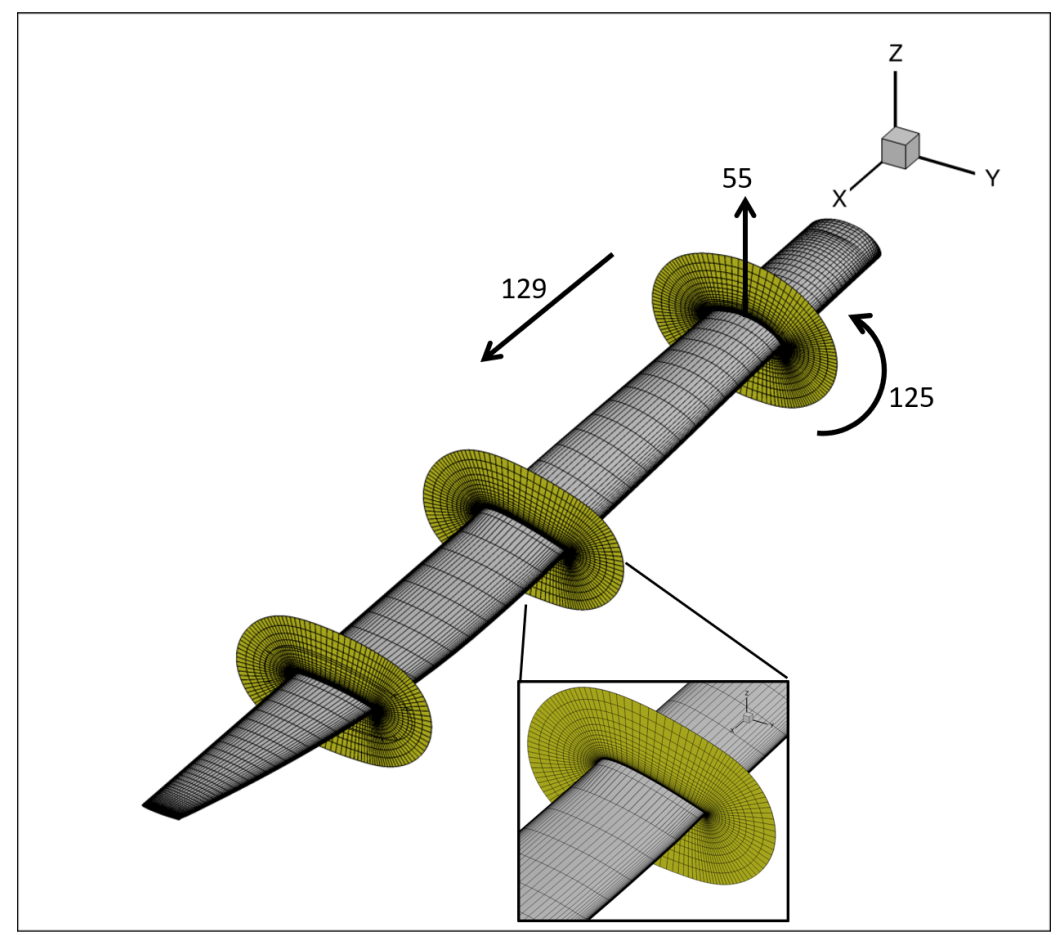

Figure 4.2: Main rotor blade mesh.

were generated by Passe in Ref. [45] using an in-house algebraic O-O grid generator. A single blade mesh, shown in Fig 4.2 , consists of 0.88 million grid points: 125 in wrap around direction, 129 in spanwise direction and 55 in the vertical direction. The eight near-body meshes have a total of 7.1 million grid points. The height of the first wall spacing was chosen such that the $y+=1$ is satisfied at a Reynolds number of 2.6 million based on the main rotor root chord and the rotor tip speed.

\subsubsection{Airframe and Propeller - FUN3D}

An unstructured CFD solver FUN3D (version 13.3) [77], developed by NASA, was chosen to solve the flow equations for the notional X2TD airframe and the propulsor. The FUN3D solver is a finite volume solver that utilizes unstructured 
meshes and solves the unsteady RANS equations. Numerous spatial and temporal schemes are available within FUN3D. In this study, the second order Roe upwinding scheme was utilized to solve the inviscid fluxes. FUN3D was run in a fully turbulent mode with Spalart-Allmaras turbulence model.

An airframe CAD geometry obtained from Passe in Ref. 45] was modified to reflect the five degree positive incidence angle of the horizontal stabilizers. An unstructured meshing technique was chosen for the notional airframe of the X2TD due to geometric complexity. The airframe containing various regions of high curvature as well as convex and concave junctions. The unstructured airframe mesh, shown in Fig 4.3 was generated using CREATE ${ }^{T M_{-}}$GENESIS Capstone software [78. The airframe surface grid consists of triangular elements. Fifty prism layers were grown from the surface to capture the boundary layer on the airframe. The thickness of the first prism layer is $7 e-5$ grid units. The airframe near-body mesh contains 15.6 million grid points.

The unstructured blade grid, shown in Fig.4.4, for the propeller was generated from blade CAD using the $\mathrm{CREATE}^{T M_{-}}$GENESIS Capstone software. The propeller consists of six blades, with 2.2 million grid points per blade, resulting in total of 13.2 million grid points. The first layer thickness was chose to be $7 e-5$ to ensure that $y+<1$ is satisfied. For consistency, one grid unit, in the airframe and propeller mesh, is equal to one root chord of the main rotor blade. 


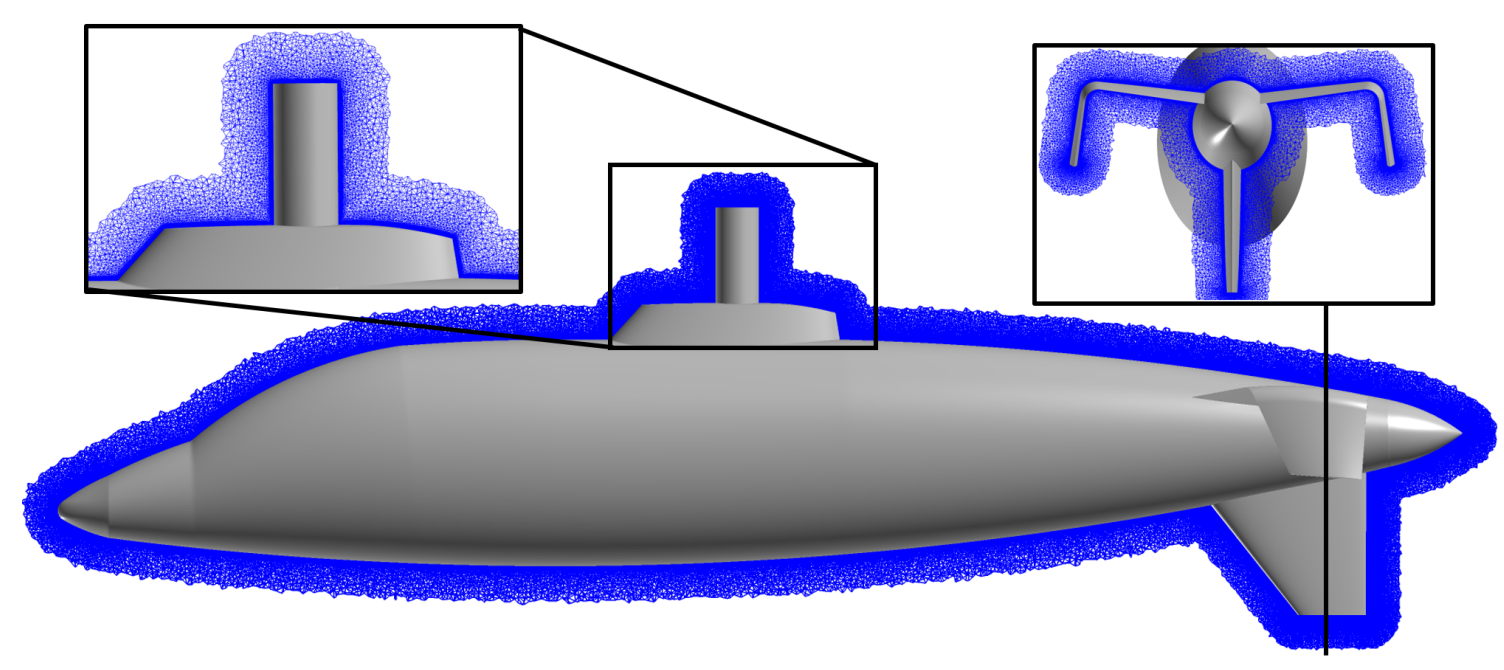

Figure 4.3: Airframe near-body surface mesh.

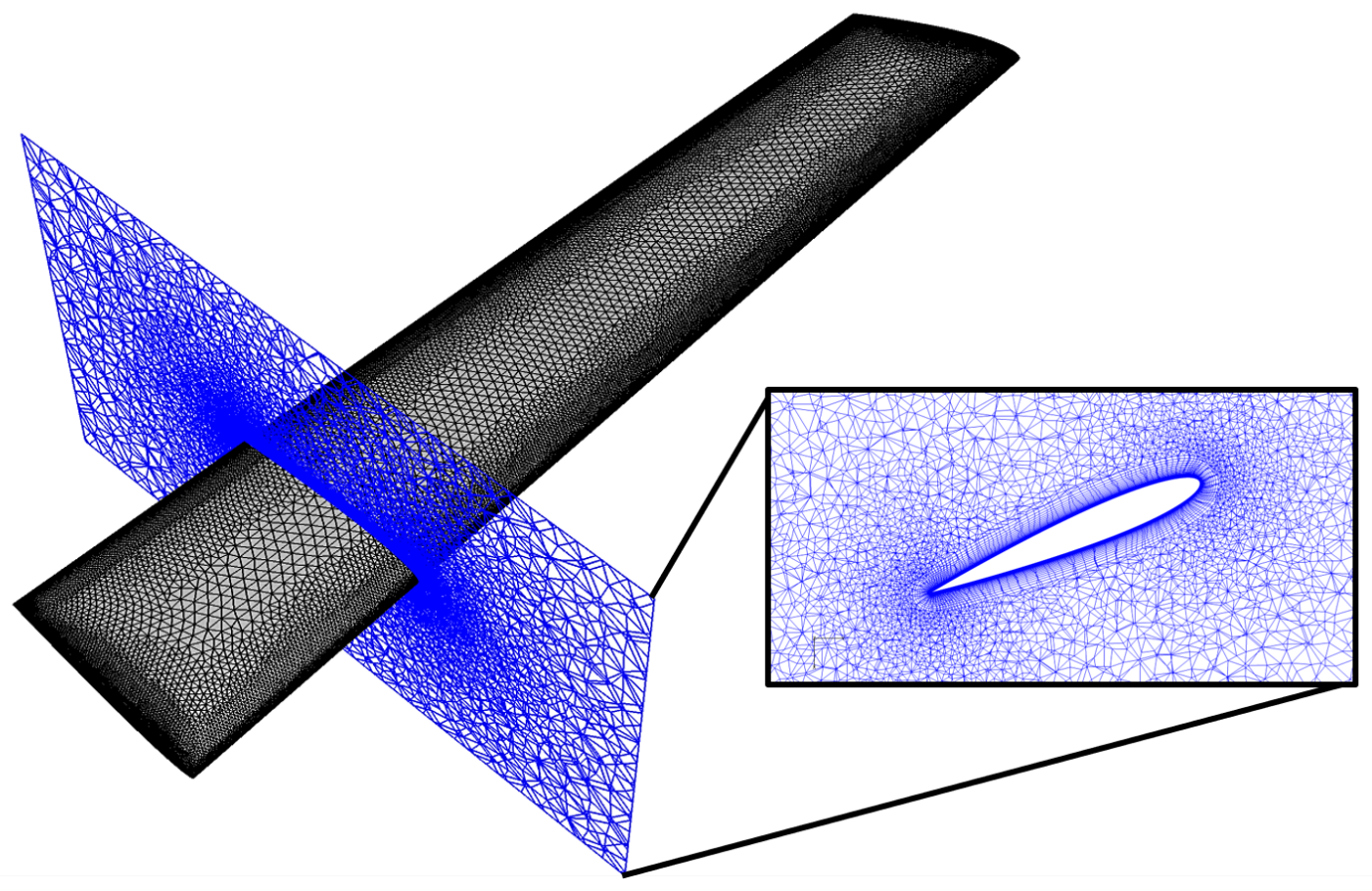

Figure 4.4: Notional X2TD auxiliary propeller near-body surface mesh. 


\subsubsection{Off-body - SAMCart}

SAMCart, a high-order Cartesian flow solver native to the Helios framework, was used to solve the flow equations in the off-body region [79]. The off-body domain extended eight rotor radii around the center of the lower rotor hub. Figure 4.5 shows a view of the mesh domain extents, from the port side of the aircraft, as well as the close up of the fixed refinement region near the aircraft. The off-body region was refined using seven nested levels, such that the finest level was ten percent of the main rotor root chord. Table 4.1 gives the grid spacing for of each refinement level. The finest region extended behind the center of the rotor shaft to capture the rotor wake structures downstream. The coarsest level defines the outer boundary

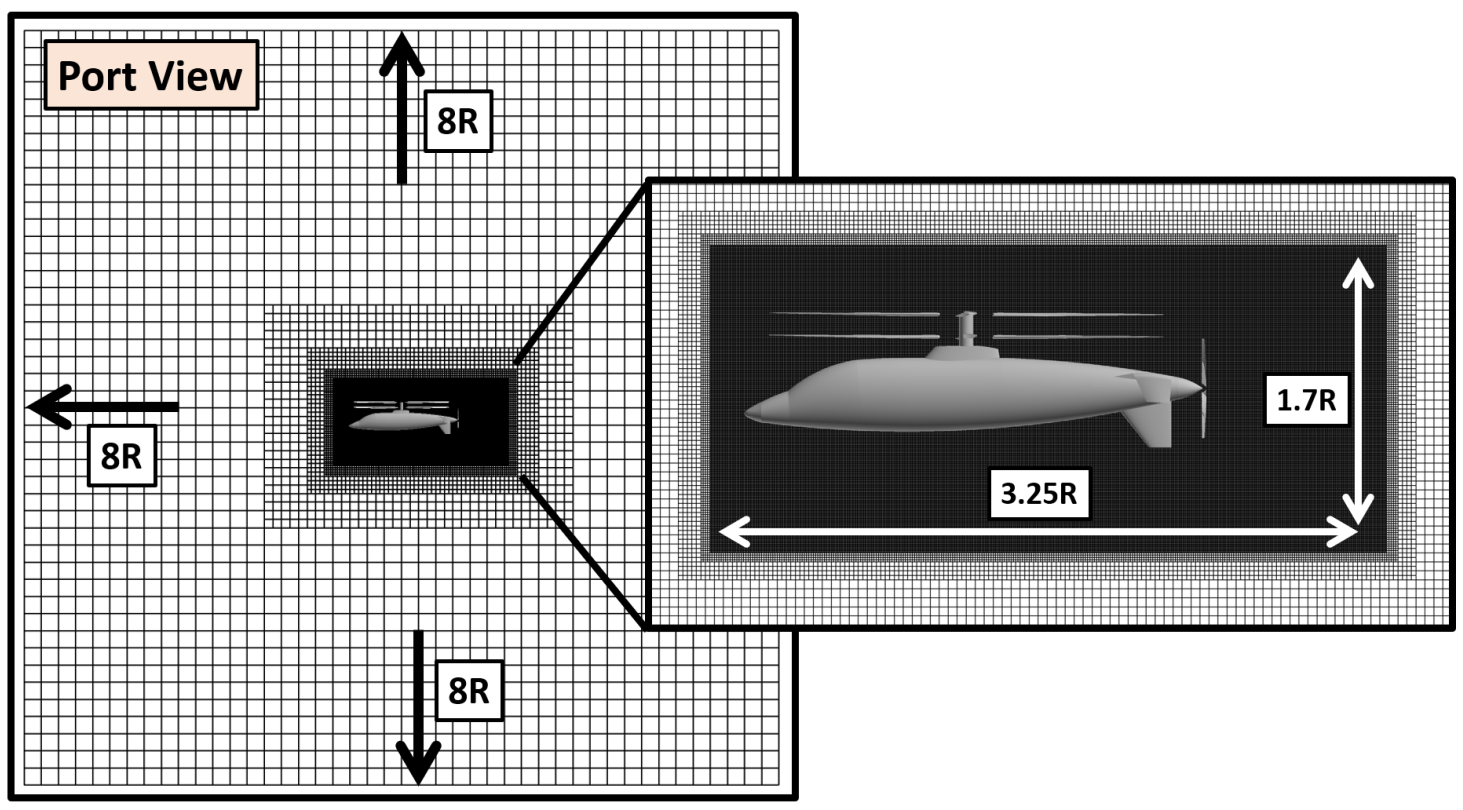

Figure 4.5: Mesh domain viewed from port side of the helicopter. 
of the computational domain. A fifth order central differencing scheme was used for the calculations of the inviscid fluxes. The viscous fluxes were discretized using the fourth order central differencing scheme. The turbulence was modeled using the Spalart-Allmaras turbulence model with the default settings. Eight sub-iterations were used for SAMCart to achieve a drop of roughly 2 orders. A total of 115 million mesh points made up the off-body Cartesian domain.

Table 4.1: Grid spacing per level in terms of root chords for the off-body Cartesian mesh.

\begin{tabular}{cc}
\hline \hline Off-Body Refinement Level & Grid Spacing \\
\hline Level 1 & 6.55 \\
Level 2 & 3.27 \\
Level 3 & 1.63 \\
Level 4 & 0.819 \\
Level 5 & 0.409 \\
Level 6 & 0.205 \\
Level 7 & 0.102 \\
\hline \hline
\end{tabular}

\subsection{Blade Motion and Deformation}

This section describes the blade deformation within the CFD solver. The main rotor blades elastically deform as they travel around the azimuth. The main rotor blades deform according to the blade elastic deformations, obtained from the com- 
prehensive analysis solver. These blade deformations include three translational and three rotational transformations around the elastic axis of the blade. For the notional X2TD the elastic axis coincides with the quarter chord line. Within the Helios framework, the Melodi module enforces the blade motions and deformations [69]. There exists a capability to transfer the blade deformations directly and automatically from the comprehensive analysis solvers to Melodi, however, only RCAS and CAMRAD comprehensive analysis solvers are currently interfaced with Helios framework. Therefor, the azimuthal distribution of the spanwise elastic deformations and the quarter chord definition, from PRASADUM, were transferred to Melodi as prescribed deformations by the way of file IO.

Unlike the main rotor blades, the propeller blades were treated as rigid blade entities in the Melodi module. The propeller collective obtained from CSD, was applied to the propeller blade using the Melodi xml input, not the prescribed deformation file. For both, the main rotor system and the propeller, the twist and the chord files were provided to Melodi to obtain the non-dimensional sectional airloads in the deformed airfoil frame. 


\section{CFD/CSD Coupling Methodology}

This chapter discusses the coaxial rotor and full vehicle CFD/CSD coupling procedures used in this study. The prediction of rotorcraft airloads is a multidisciplinary task that requires the knowledge of structural dynamics of the rotor blades as well as the aerodynamics of the operational flow field [80]. Various types of CFD/CSD coupling approaches are possible including tight and loose coupling 81]. In a tight coupling, the exchange of information between the CFD and the CSD solvers occurs every time step. While, in a loose coupling approach the exchange of information occurs periodically. A loose CFD/CSD coupling approach is utilized for this study. Because this study is performed for steady level flight, it was deemed acceptable to use the loose CFD/CSD coupling approach. For flight regimes where the state of the aircraft is highly coupled to time, a tight coupling approach is recommended.

\subsection{Rotor CFD/CSD Coupling}

This section provides an overview of the conventional, loosely coupled CFD/CSD approach utilized in this study to obtain results for the isolated coaxial rotor shown

in Chapter 6. This coupling approach follows the methodology described in Refs. [9] 


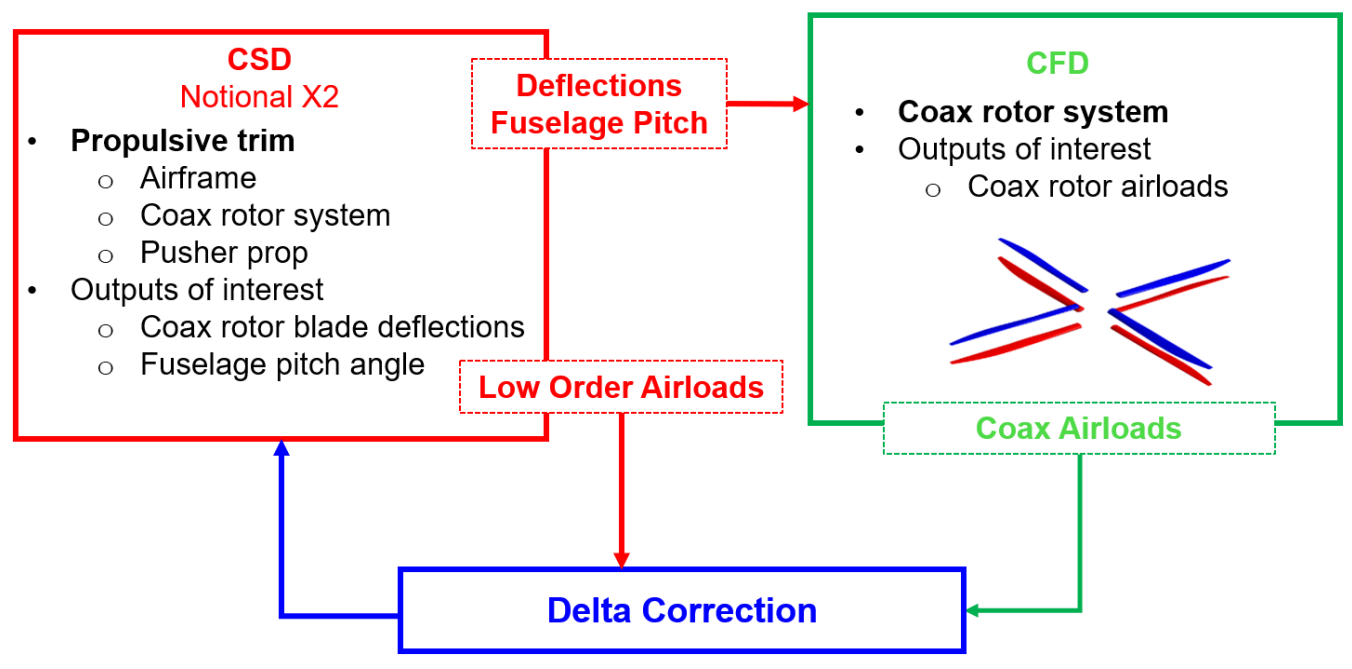

Figure 5.1: Conventional, rotor CFD/CSD coupling schematic.

and $[16]$. Because this conventional approach transfers the information pertaining only to the main rotor between the CFD and CSD solvers, from here on out it will be referred to as a "rotor" CFD/CSD coupling approach. Figure 5.1 shows the schematic of the rotor $\mathrm{CFD} / \mathrm{CSD}$ coupling approach. At the beginning of the procedure, a propulsive trim, described in Chapter 3, is performed using an in-house comprehensive analysis solver.

The trim procedure solves for the main rotor elastic blade deformations at specified locations along the blade. The elastic blade deformations and the pitch attitude of the vehicle are the inputs into the CFD simulations. Because the CSD and the CFD codes are not directly interfaced, the data transfer is done using manual file IO method. The main rotor elastic deformations from the CSD are manually transferred to the location of the CFD simulation. Once the CFD simulation has 
completed 2 revolutions, the non-dimensional airloads in deformed airfoil frame, are manually transferred back from the CFD to the location of the CSD solver. A delta coupling procedure, given in Eqn 5.1 , is utilized to obtain the difference between the CFD airloads and the low order CSD airloads.

$$
C S D^{i+1}=L O^{i+1}+\left(C F D^{i}-L O^{i}\right)=L O^{i+1}+\Delta^{i}
$$

The difference between the high-fidelity CFD airloads and the low order CSD airloads is treated as a correction which is applied to the next trim iteration. Once a new trim state is obtained using the delta correction, the new CSD elastic blade deformations are manually transferred back to the location of the CFD code. A new CFD simulation is performed. It was decided not to restart from an old CFD solution due slower convergence which stems from initial transients. Thus, a completely new CFD cycle was started after every CFD/CSD coupling exchange. Take note, for this study only the main rotor system is present in the CFD simulations for this type of coupling. However, it is common to also include the airframe and/or prop in CFD, as shown in Ref. [82].

\subsection{Full Vehicle CFD/CSD Coupling}

The rotor CFD/CSD methodology, described in previous section, was utilized for the coaxial rotor portion of the results, presented in Chapter 6. This section discusses the full vehicle CFD/CSD coupling procedure utilized for the full vehicle and the interactional aerodynamic portions of the results, discussed in Chapters 7 and 8 , respectively. Figure 5.2 shows the schematic of the full aircraft trim procedure. The 
key difference between the rotor CFD/CSD coupling procedure, shown in Fig 5.1 , and the full vehicle CFD/CSD coupling approach is the trimming of the auxiliary propeller and the presence of the airframe and propeller in the CFD simulations.

The full vehicle CFD/CSD coupling procedure is performed as follows. First, a wind tunnel trim was performed for the isolated notional X2TD auxiliary propeller to obtain the propeller collective for a given flight condition. The three targets for the wind tunnel trim are the propeller power, pitching moment and roll moment. The wind tunnel target propeller power was determined from the power curve for the actual X2TD propeller given in the Ref. [46]. Both, the pitching moment and the roll moment targets were assumed to be zero for the wind tunnel trim. The auxiliary propeller collective resulting from the wind tunnel trim was retained for

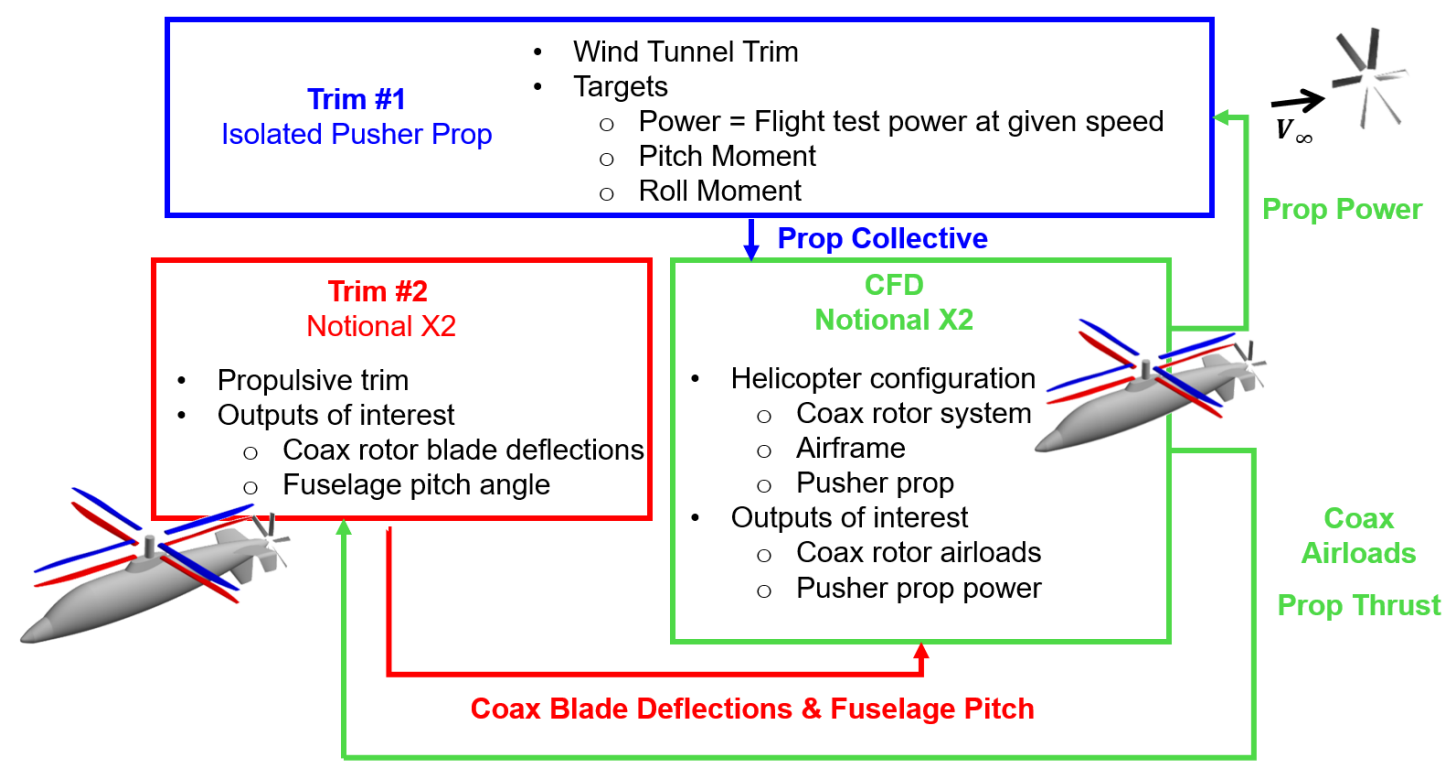

Figure 5.2: Full vehicle CFD/CSD coupling schematic. 
CFD simulations. Second, a propulsive trim was performed to obtain main rotor elastic blade deflections and pitch attitude for the notional X2TD vehicle. The propulsive trim included the full notional X2TD vehicle, as described in Chapter 3. Note that in the first trim iteration, the propeller thrust in the lookup-table is obtained from a simplified expression, $P=T V$. However, for all of the consecutive iterations the propeller thrust is obtained from the CFD simulations. Then, the main rotor blade elastic deformations, vehicle pitch attitude and propeller collective are incorporated into the CFD simulations.

Once the CFD simulations has completed 2 main rotor revolutions the main rotor sectional airloads, mean airframe airloads and the mean propeller yaw hub moment are transferred to CSD solver. The mean propeller power is extracted from the CFD propeller yaw moment. A cumulative delta difference, starting at the initial coupling cycle is calculated and applied to the CSD propeller airloads, the propeller is then re-trimmed to the target power and a new propeller collective is obtained. The full aircraft speed schedule mean propeller thrust from the most recent CFD run is entered into the propeller speed schedule for the full aircraft trim. The delta correction discussed in previous section and given by Eq.5.1, was utilized to correct the CSD low order main rotor airloads. A propulsive trim is once again performed with the delta correction. Then the main rotor deflections, fuselage pitch attitude and propeller collective are manually transfered back to the CFD solver. The data transfer between CFD and CSD solvers continues until convergence is achieved for the rotor controls, sectional airloads and propulsor power. 


\subsection{CFD/CSD Coupling Validation}

The rotor CFD/CSD coupling procedure discussed in this chapter was validated using the existing full scale UH-60A rotor wind tunnel test data, obtained May 2010 in the 40-by-80-Foot NFAC wind tunnel Ref. [83]. An in-depth discussion of the wind tunnel test and the results is given in Ref. [83]. One of the four UH-60A rotor blades was instrumented with 235 pressure transducers, distributed along the chord at the nine spanwise locations. The sectional normal force, chordwise force and pitching moment along the blade were integrated from the chordwise pressure distributions. The wind tunnel case, 5240, is representative of a high-speed level flight condition with advance ratio of $\mu=0.3$, blade loading of $C_{T} / \sigma=0.09$ and advancing tip Mach number $M_{\text {Adv.Tip }}=0.65$. The Helios/OVERFLOW CFD solvers were coupled with the in-house comprehensive analysis solver. Wind tunnel trim was performed with four harmonics and eight blade modes, trimming the rotor to target thrust, rolling and pitching moments. Figure 5.3 shows the sectional normal force and pitching moment at two spanwise stations along the blade, $r / R=0.225$ and $r / R=0.865$. The CFD/CSD predicts reasonably well the normal force trends. There is negative loading on the outboard stations of the blade, in the second quadrant, due to decreased angle of attack resulting from the the need to maintain equilibrium of the roll moments. However, the magnitude of the negative normal force peek is under-predicted. The normal force trends on the retreating side, quadrants 3 and 4, matches reasonably well to the test data. These results were approved for public release and presented at the 2017 UH-60A Airloads workshop 84. 

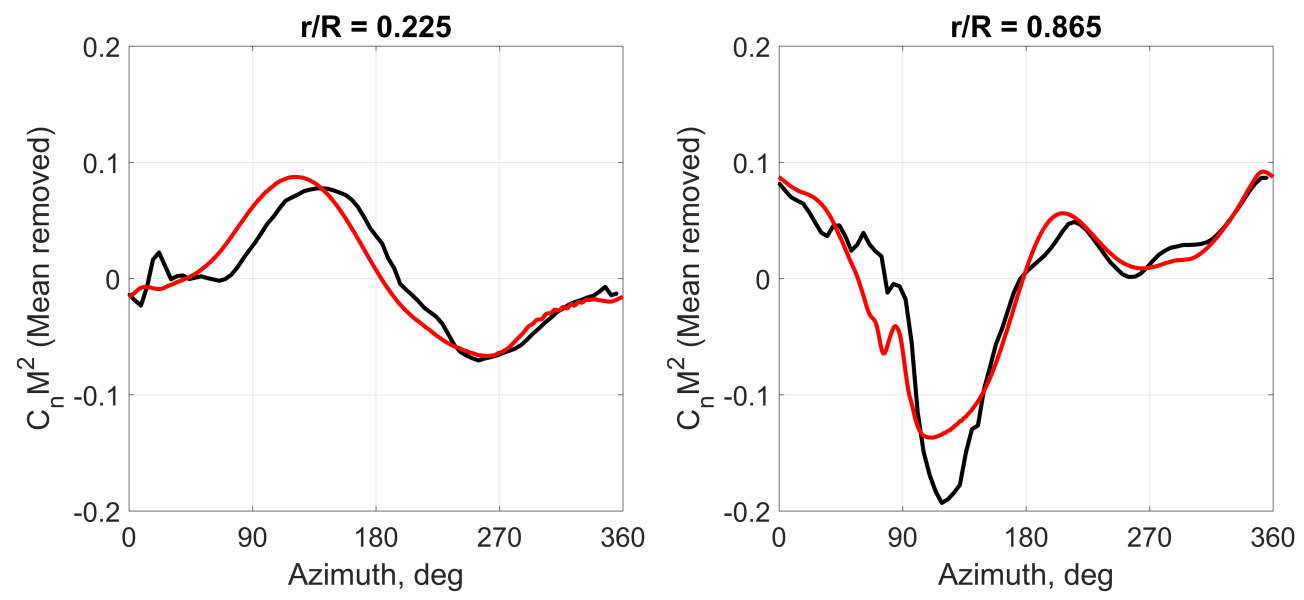

(a)
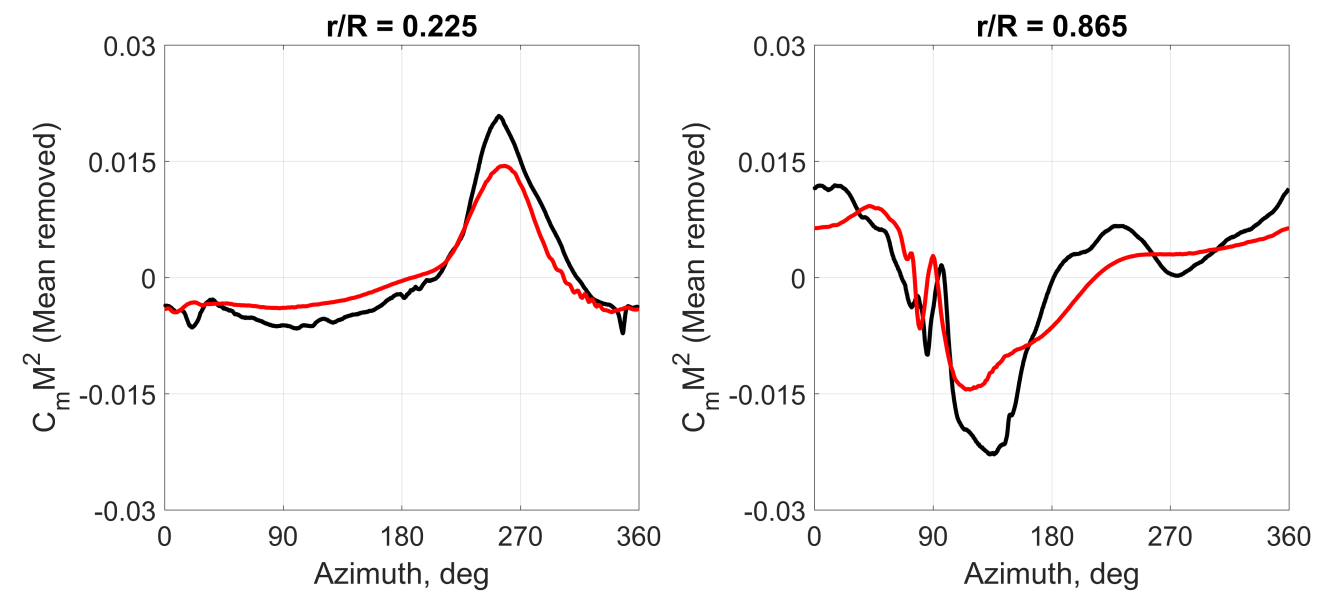

(b)

Figure 5.3: Comparison of predicted CFD/CSD airloads with the wind tunnel test data for UH-60A rotor. 


\section{Results Part I: Coaxial Rotor CFD/CSD Coupling}

This chapter discusses the results from the coaxial rotor CFD/CSD coupling study. In this study, the CFD simulations included only the coaxial rotor system, consisting of an upper rotor, rotating counter-clockwise, and the lower rotor, rotating clockwise. Figure 6.1 shows the coaxial rotor system utilized for this study. Take note that the airframe and pusher propeller were not present in the CFD simulations. However, a full vehicle propulsive trim was performed in the CSD and a delta coupling procedure was used to correct the low order rotor CSD airloads. The results shown in this section are from converged coaxial CFD/CSD coupling iterations.

The first objective of the coaxial rotor CFD/CSD coupling study was to identify and explain the effect of forward flight speed on the trim parameters, blade deflections and airloads. Table 6.1 shows the five forward flight speeds that were investigated. The LOS value for the each flight condition was obtained from a linear interpolation of the flight test data presented in Ref. [5]. The second objective was to analyze the rotor-rotor interactions at 50 knots and explain the interactional effects on the trim parameters and performance of the rotor. The same CFD/CSD coupling procedure, as above, was performed. However, for the non-interactional case the CFD runs were performed with isolated rotors. 

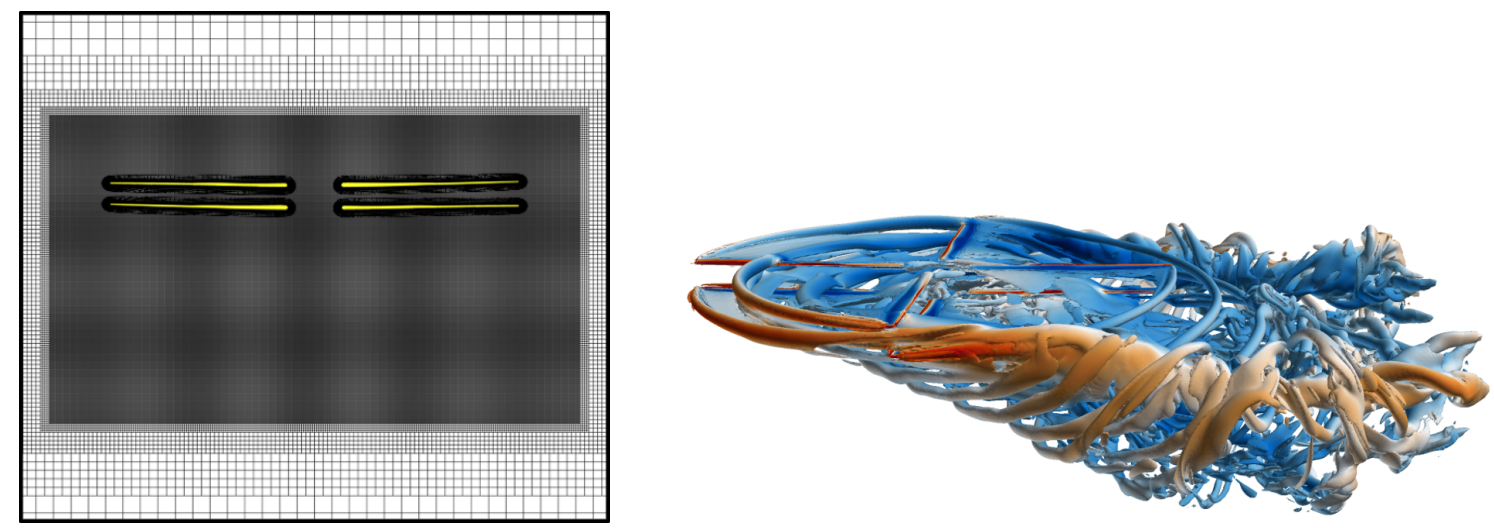

Figure 6.1: The coaxial CFD/CSD coupling mesh system and Q-criterion flow visualization for coaxial rotor at 50 knots.

Table 6.1: Coaxial CFD/CSD coupling forward flight speeds.

\begin{tabular}{ccccc}
\hline \hline$V_{\infty}$ (knots) & $\mu$ & MR RPM & $M_{A D V}$ & $\operatorname{LOS}(\% R)$ \\
\hline \hline 50 & 0.14 & 446 & 0.64 & 3.75 \\
\hline 100 & 0.27 & 446 & 0.71 & 7.50 \\
\hline 150 & 0.41 & 446 & 0.79 & 11.3 \\
\hline 200 & 0.55 & 446 & 0.87 & 15.0 \\
\hline 225 & 0.65 & 423 & 0.88 & 16.9 \\
\hline \hline
\end{tabular}




\subsection{Main Rotor Controls and Vehicle Pitch Attitude}

This section discusses the effect of flight speed on the trim configuration of the vehicle. The control angles and the vehicle pitch attitude are examined at various flight speeds given in the Table 6.1. Figure 6.2 and Table 6.2 show the coaxial rotor controls and fuselage pitch attitude. The following sections will analyze and explain the trends shown in Fig.6.2(a) and (b).

\subsubsection{Rotor Collective $\left(\theta_{0}\right)$ and Fuselage Pitch}

This subsection examines the upper and lower rotor collective and vehicle pitch attitude as a function of flight speed for final CFD/CSD coupling iterations. Between 50 and 100 knots, the vehicle pitch attitude changes from a tilt fore to a tilt aft orientation. The pusher propeller begins to contribute a substantial amount of the propulsive thrust to counteract the vehicle drag. This results in a decrease of the collective angle, since the rotor does not need to provide both the propulsive thrust and the lifting force. Note that in the CSD, the pusher propeller thrust is prescribed in a look-up table and the vehicle is trimmed to account for the pusher propeller thrust, as described in the Methodology section.

From 100 knots to 150 knots, the rotor collective decreases and the vehicle pitch attitude also slightly decreases. The collective decreases because the rotor is offloaded since the pusher propeller is providing the propulsive thrust to counteract the vehicle drag. However, the vehicle does not need to tilt back as much compared to 100 knots, to counteract the propulsive force from the pusher prop in the trim 


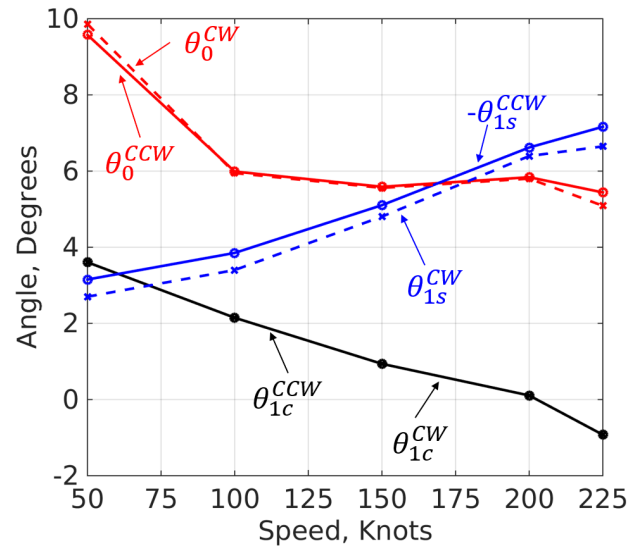

(a)

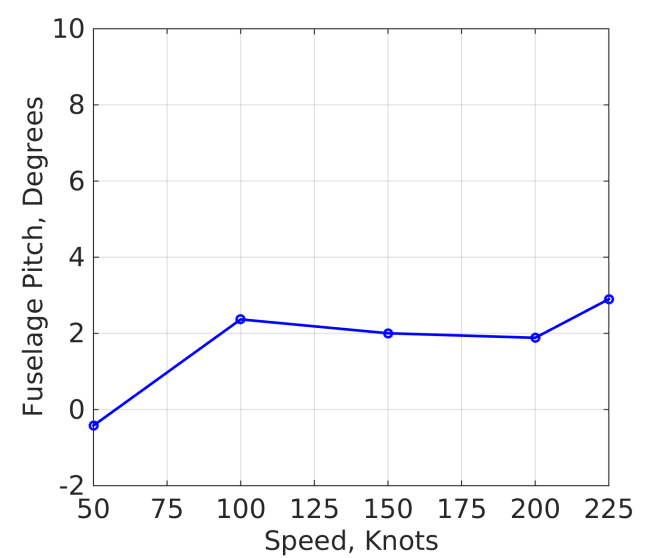

(b)

Figure 6.2: The final coaxial CFD/CSD main rotor controls (a) and vehicle pitch attitude (b) at various speeds.

Table 6.2: The final coaxial CFD/CSD controls and vehicle pitch attitude at various speeds. The control angles and the vehicle pitch attitude are shown in degrees.

\begin{tabular}{c|c|cc|cc|cc}
\hline \hline$V_{\infty}$ (knots) & $\alpha_{F}$ & $\theta_{0}^{C C W}$ & $\theta_{0}^{C W}$ & $\theta_{1 c}^{C C W}$ & $\theta_{1 c}^{C W}$ & $\theta_{1 s}^{C C W}$ & $\theta_{1 s}^{C W}$ \\
\hline \hline $\mathbf{5 0}$ & -0.42 & 9.58 & 9.86 & 3.61 & 3.61 & -3.15 & 2.70 \\
\hline $\mathbf{1 0 0}$ & 2.37 & 5.99 & 5.95 & 2.15 & 2.15 & -3.85 & 3.40 \\
\hline $\mathbf{1 5 0}$ & 2.00 & 5.59 & 5.56 & 0.94 & 0.94 & -5.10 & 4.81 \\
\hline $\mathbf{2 0 0}$ & 1.88 & 5.84 & 5.80 & 0.11 & 0.11 & -6.62 & 6.40 \\
\hline $\mathbf{2 2 5}$ & 2.90 & 5.44 & 5.09 & -0.92 & -0.92 & -7.16 & 6.65 \\
\hline \hline
\end{tabular}


process, because the drag on the rotor is substantially high.

Fromm 150 knots to 200 knots, the rotor collective slightly increases. At 200 knots, the rotor wake is convected straight back thus each rotor is forced to operate in it's own wake. The wake-rotor interactions result in decrease of rotor thrust, therefore the rotor collective needs to be increased at this operational speed. From 200 knots to 225 knots, the rotor wake is convected through the upper rotor, reducing the downwash.

There is less than one percent difference between the upper and the lower rotor collectives for the 100, 150 and 200 knots, shown in Fig.6.2(a). However, at flight speeds of 50 knots and 225 knots, the difference between the upper and the lower collective increases to more than three percent. At 50 knots, the lower rotor is trimmed to a higher collective than the upper rotor and the vehicle has a forward tilt, as shown in Fig $6.2(\mathrm{~b})$. The wake from the upper rotor is convected down and backwards, through the aft portion of the lower rotor disk, as shown in Fig.6.1. The wake from the upper rotor impinges on the lower rotor, causing lower rotor to loose thrust, which in turn is forces the lower rotor to trim to higher collective in order to maintain the required thrust. A similar but opposite scenario occurs at 225 knots. At 225 knots, the upper rotor collective is higher than the lower rotor collective and the vehicle is titled aft. The backward tilt of the vehicle forces the wake of the lower rotor to be ingested into the upper rotor, causing the upper rotor to loose lift and needing to operate at a higher collective. 


\subsubsection{Cyclic Pitch $\theta_{1 s}$ and Cyclic Pitch $\theta_{1 c}$}

Rotor control, $\theta_{1 s}$, is associated with lift-offset, shown in Table 6.1. Lift-offset is a measure of the displacement of the thrust vector from the center of the hub. In an SMR configuration, the roll moment balance is achieved from the equilibrium of the thrust on advancing and retreating sides of the disk. The time-averaged thrust acts at the center of the rotor hub. The thrust produced on the advancing side is limited by the amount of thrust produced on the retreating side. In a coaxial configuration, the roll moment equilibrium is achieved from the balance of roll moments of each rotor. This offloads the retreating side and allows the advancing side to produce more lift. As the speed increases, the LOS also increases, resulting in an increase in the $\theta_{1 s}$. An independent CSD study was conducted to observe the effect of LOS at various speeds. Without lift offset, thrust vector acts at the axis of rotation, the pitch angle on the advancing side was minimum. As the lift offset increased the pitch angle on that advancing side also increased. The cyclic control, $\theta_{1 c}$, is associated with the control of the pitch on the fore and the aft of the rotor disk. As the speed increases, the $\theta_{1 c}$ decreases, which is indicative of a shift in pitch bias towards the advancing side of the rotor disk

\subsection{Blade Pitch and Flap}

This section shows the effect of the forward flight speed on the blade tip pitch and blade tip flap angles. It follows as an extension of the previous section where the collective and the cyclic pitches were given in the Table 6.2 and Fig 6.2(a). 
The five forward flight speeds discussed in this section are shown in Table 6.1. A positive blade pitch is defined as a pitch-up rotation about the blade quarter chord. A positive flap is defined as a flap-up motion of the blade.

Figure 6.3 shows the time-history of the elastic blade tip pitch deflection for the speeds given in Table 6.1. The upper rotor blade tip pitch is shown in Fig. 6.3(a), while the lower rotor blade tip pitch is shown in Fig.6.3(b). An increase in forward flight speed results in a decrease of the minimum blade pitch on the advancing side. This is due to an increase in the LOS and the decrease in the $\theta_{1 s}$, as shown in Fig 6.2(a) The location of the minimum blade pitch on the advancing side shifts to an earlier azimuthal location as the speed increases. This is a result of the decrease in the $\theta_{1 c}$, Fig 6.2 (a). At the 50 knots speed, the blade pitch on the advancing side differs significantly compared to the rest of the speeds, which is a reflection of the trim state at that speed. The pusher propeller only provides roughly 18 pounds of thrust at 50 knots, therefore the rotor needs to provide enough thrust to counteract the weight from the vehicle as well as the drag of the vehicle, resulting in a high collective.

Figure 6.4 shows the time-history of the rotor elastic blade tip flap deflection for the speeds given in the Table 6.1. The upper rotor, Fig.6.4(a), experiences an increase in the flap-down motion on the aft portion of the rotor disk as a result of increase in speed.

The flap-up motion on the advancing and retreating sides, at $\psi=90$ and $\psi=270$, is predominantly associated with roll moment of the rotors. For a single main rotor, the roll moment equilibrium is achieved by utilizing both, the advancing 


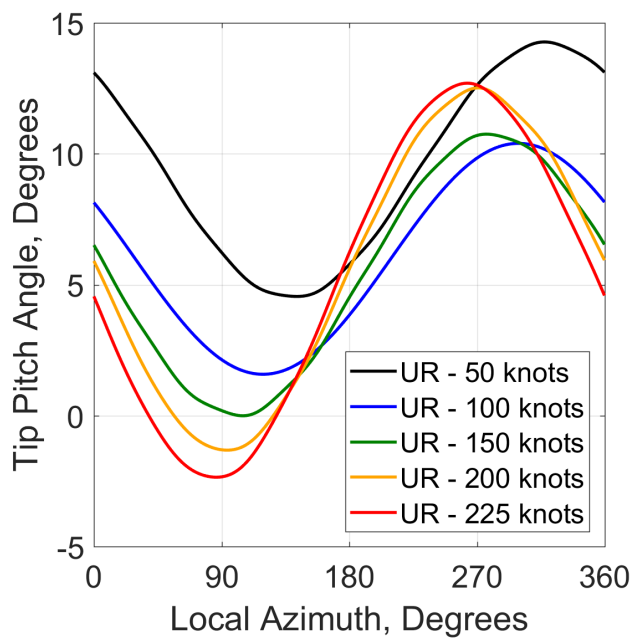

(a)

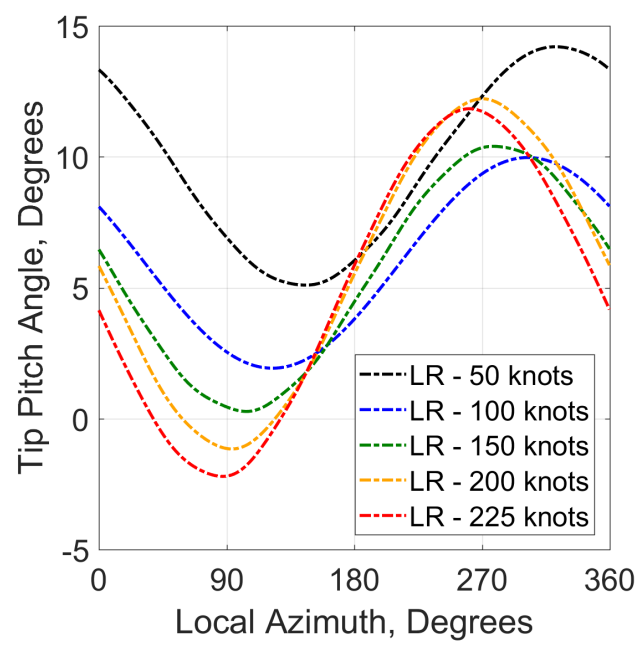

(b)

Figure 6.3: Upper rotor and lower rotor tip pitch deflections for coaxial and full vehicle CFD/CSD coupling at a range of forward flight speeds.

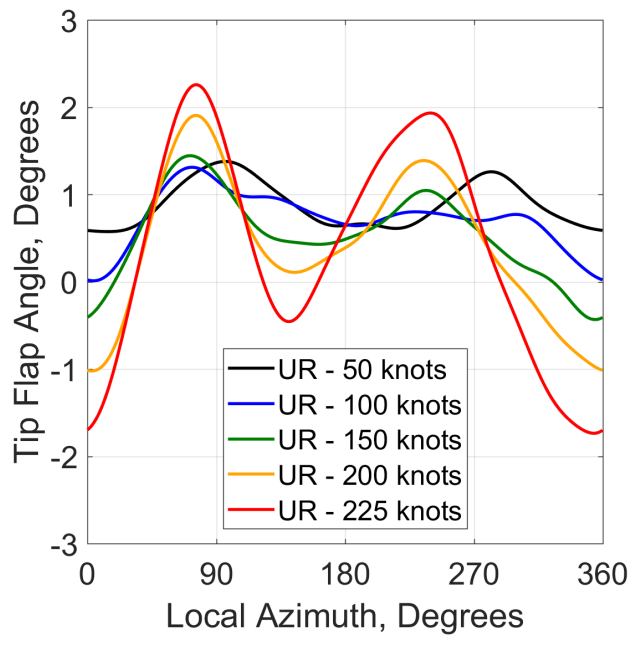

(a)

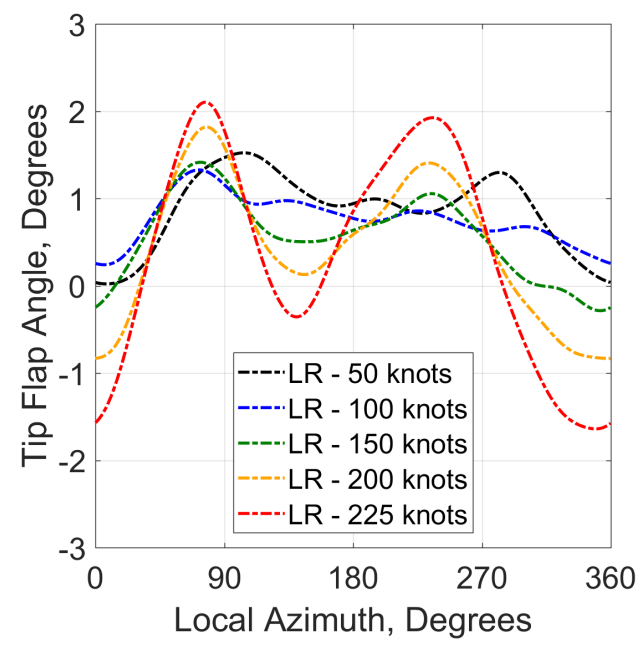

(b)

Figure 6.4: Upper rotor and lower rotor tip flap deflections for coaxial and full vehicle CFD/CSD coupling at a range of forward flight speeds. 
and the retreating side. In contrast, for the coaxial rotor configuration, the roll moment equilibrium can be achieved by utilizing the advancing sides of the upper and the lower rotors using lift-offset. As previously mentioned, lift-offset is a measure of displacement of the thrust vector from the rotational axis of the rotor. At 50 knots, the lift-offset is relatively small, $L O S=3.75 \% R$, resulting in the advancing and the retreating sides of the rotors are producing roughly similar roll moments, as shown in Fig 6.4(a) and Fig.6.4(b). As flight speed increases so does the LOS, resulting in higher flap-up and more roll moment on the advancing side.

\subsection{Rotor Airloads}

This section presents the airloads for various speeds and conditions given in Table 6.1. Figure 6.5 shows contour plots of the non-dimensional normal force, pitching moment and chordwise force distributions on the upper rotor, rotating counter-clockwise. The airloads for the lower rotor, rotating clockwise, are shown in Fig. 6.6. The airloads presented in this section are shown in the deformed airfoil frame. The normal force is positive up, pitching moment is positive for pitch-up and chordwise force is positive from the trailing edge to the leading edge.

The upper and lower rotor normal force distribution for forward flight speeds of $50-225$ knots are shown in the Fig. 6.5(a) and Fig. 6.6(a), respectively. A redistribution of normal force over the rotor disk is observed from the comparison of the normal force contours across the range of forward flight speeds. At the lowest speed, 50 knots, the normal force is distributed around the rotor disk, with highest 


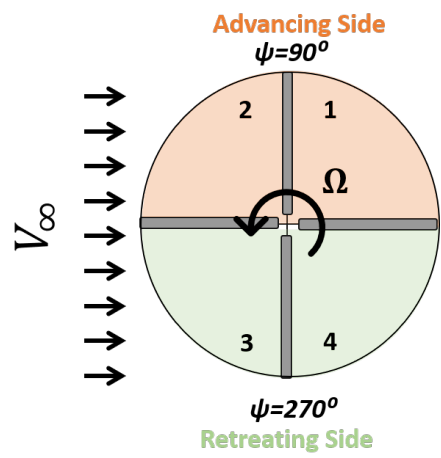

(a)
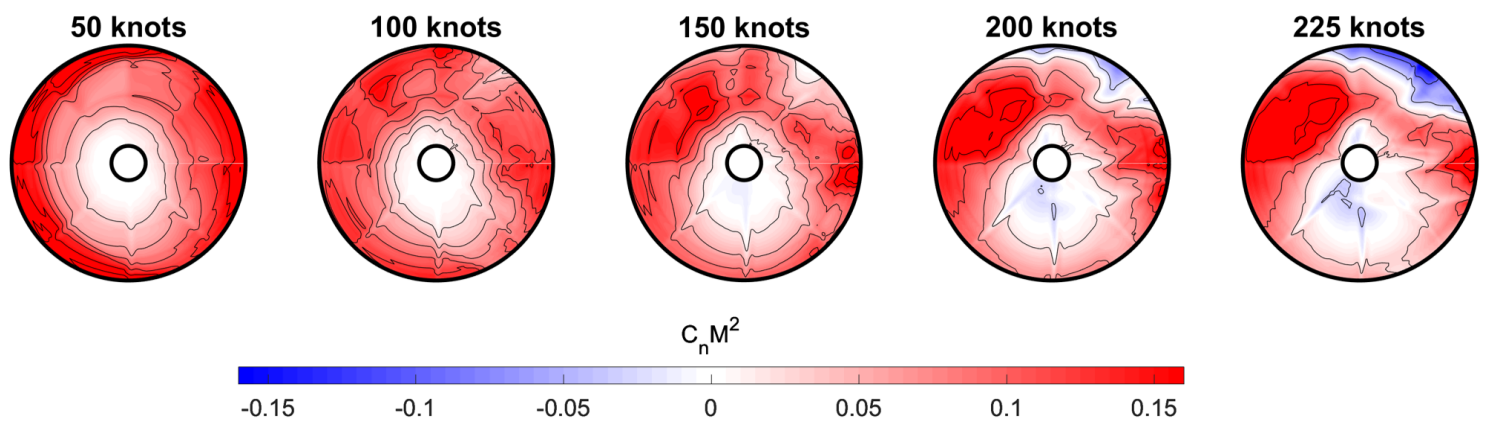

(b)
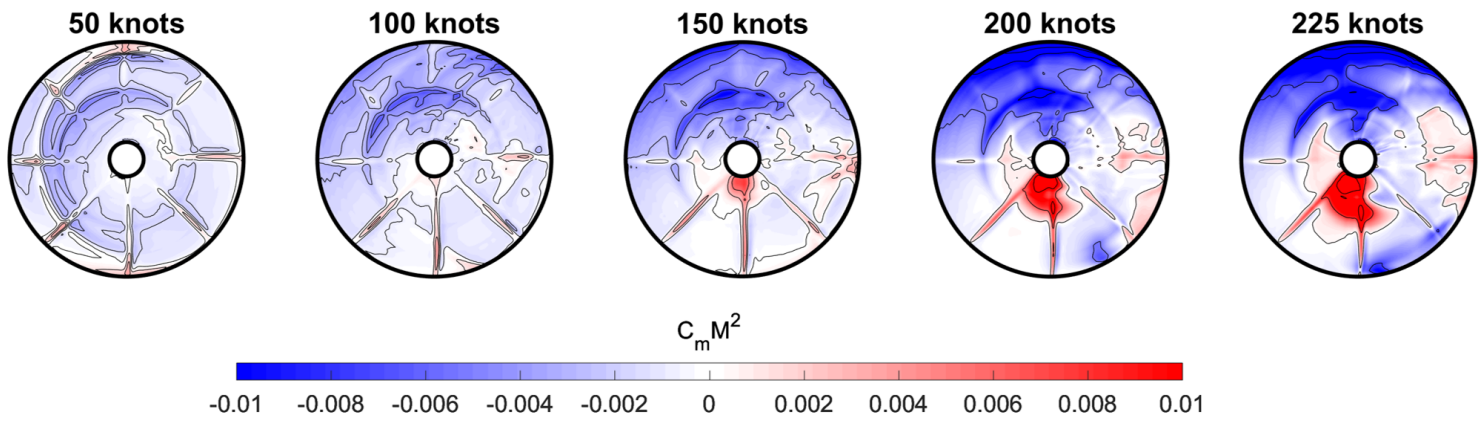

(c)
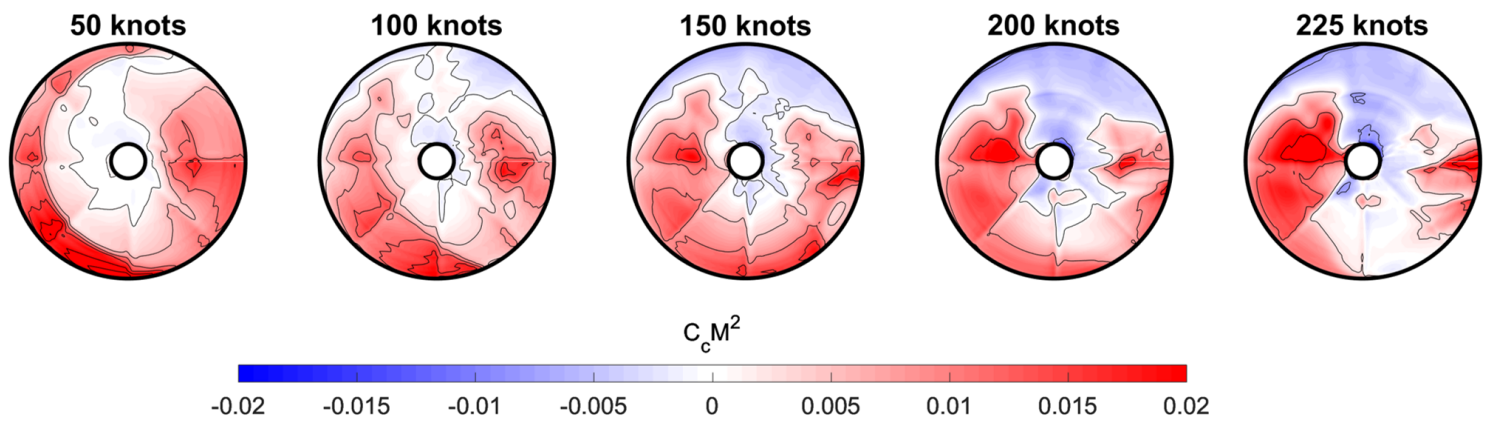

(d)

Figure 6.5: The upper rotor airloads for a range of speeds. 


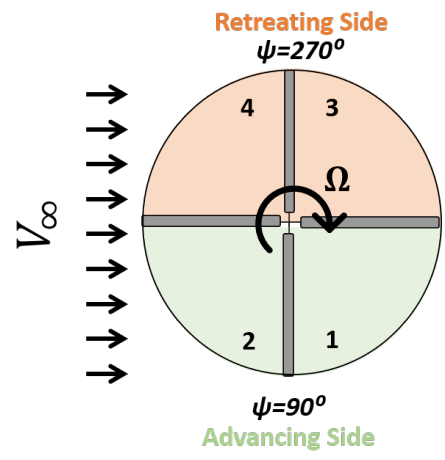

(a)
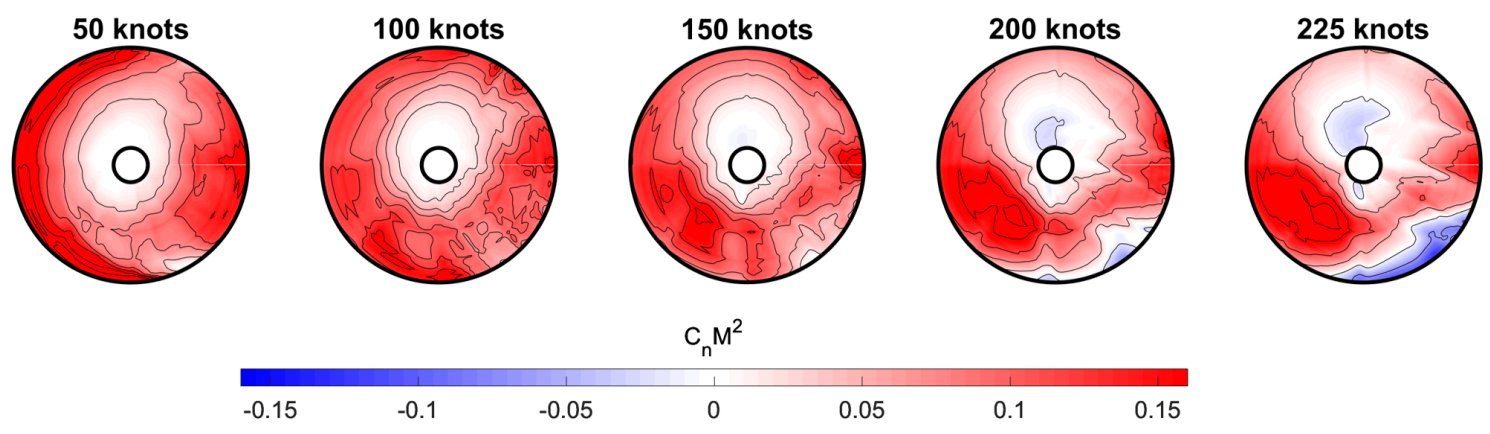

(b)
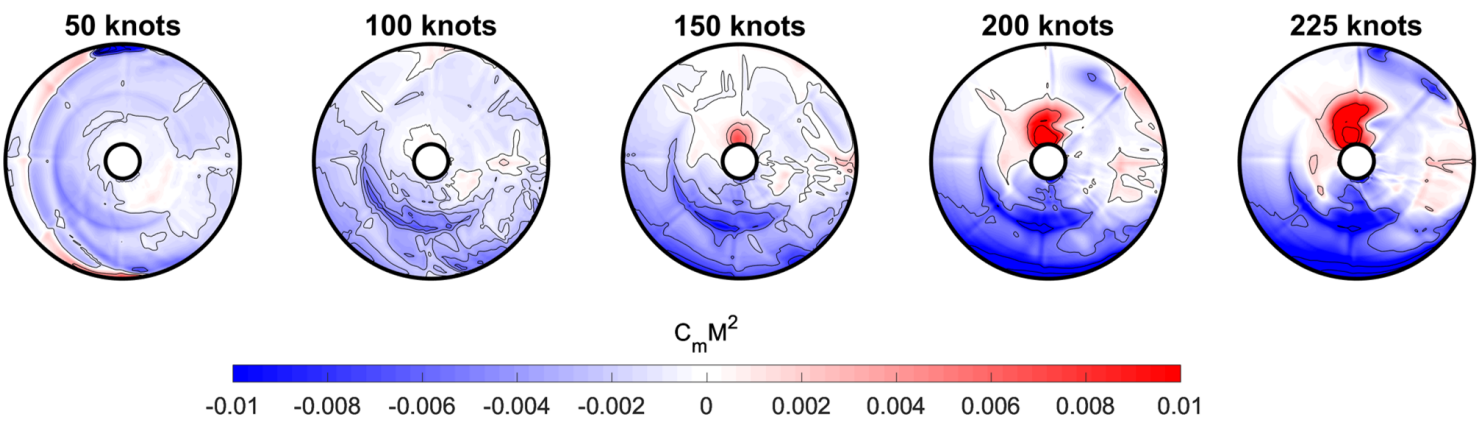

(c)
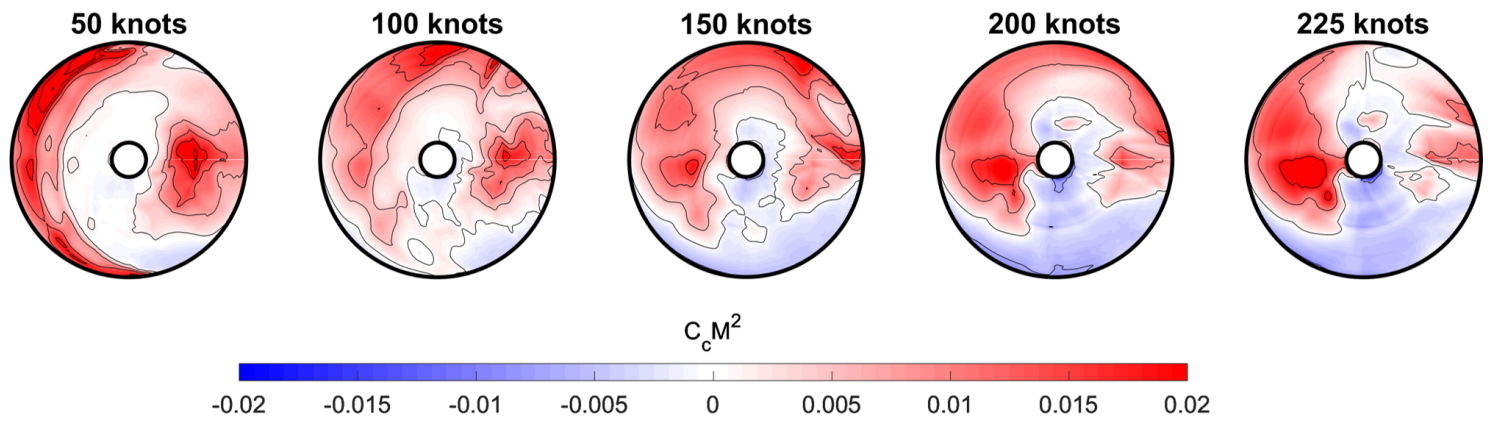

(d)

Figure 6.6: The lower rotor airloads for a range of speeds. 

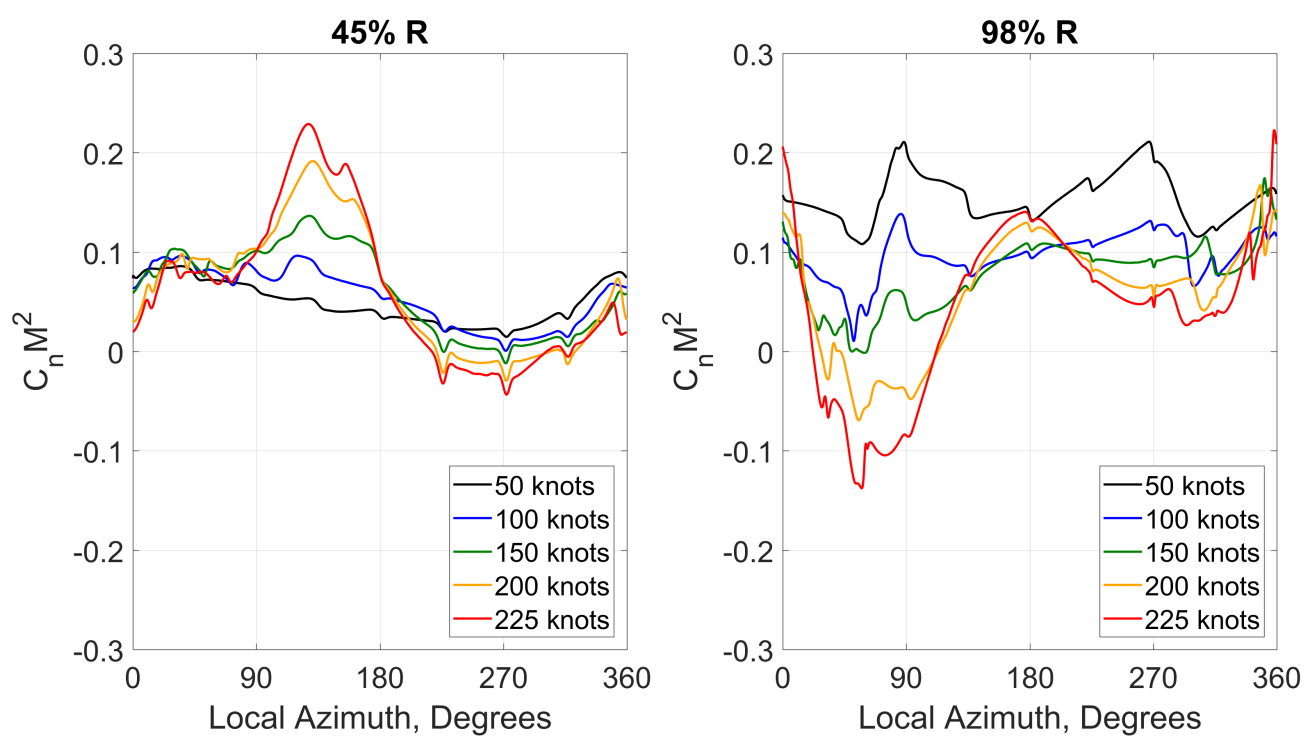

(a) Upper Rotor
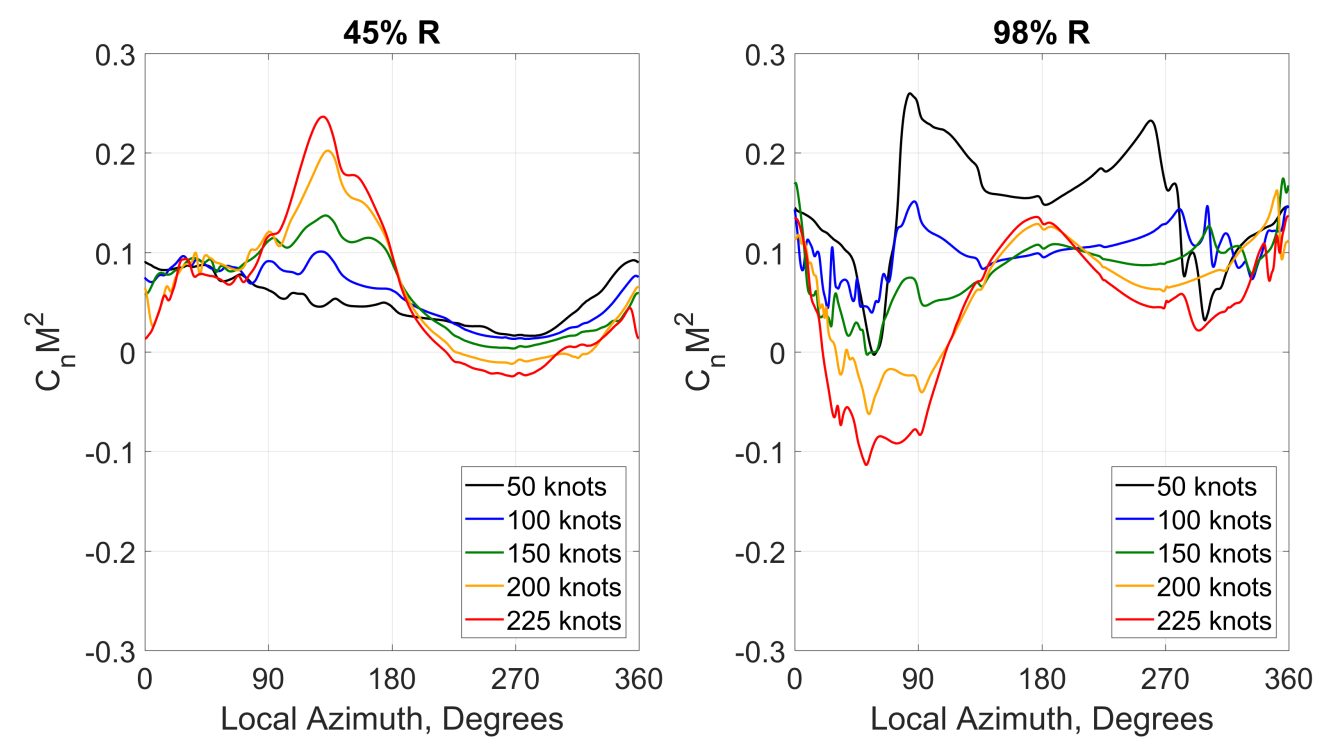

(b) Lower Rotor

Figure 6.7: The upper(a) and lower(b) rotor sectional normal force for a range of speeds. 

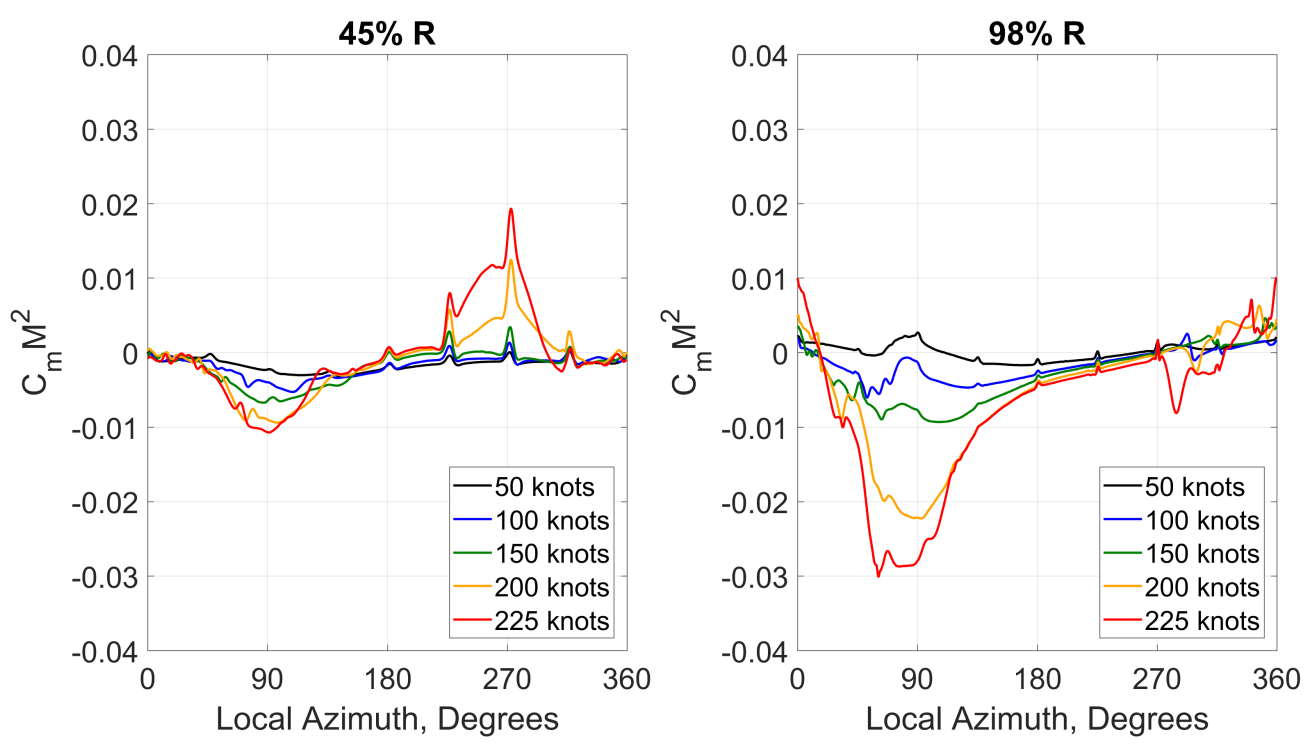

(a) Upper Rotor
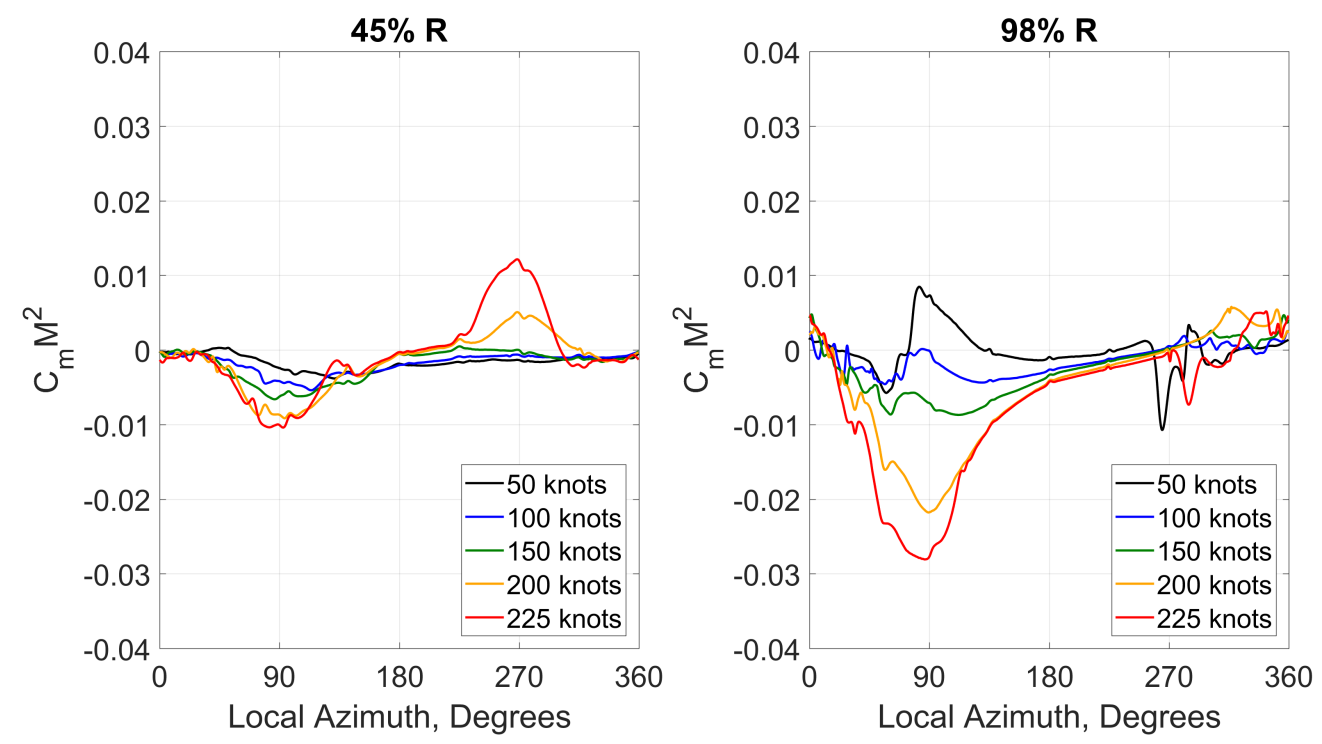

(b) Lower Rotor

Figure 6.8: The upper (a) and lower rotor (b) sectional pitching moment for a range of speeds. 
normal force along the outer portion of the blade. At the highest speed, most of lift is distributed on the fore and aft portions of the rotor disk, biased toward the advancing side. The redistribution of lift towards the fore and aft of the rotor disk observed at the intermediate speeds, 100 - 200 knots, is related to the decrease in the cyclic pitch $\theta_{1 c}$, which causes a shift in the minimum pitch towards the advancing side as shown in Fig 6.3 . Starting at a 100 knots, decrease in normal force on the advancing blade tip, is observed in the first quadrant. This region of negative normal force on the advancing side, grows in both azimuth and span, with increased speed. This is a consequence of the increase of the cyclic pitch $\theta_{1 s}$, which increases with forward flight speed. Even though there is an increase in the lift-offset and an offloading of the retreating side, the cyclic pitch $\theta_{1 s}$ is still substantially high. The magnitude of the $\theta_{1 s}$ and the negative twist on the tip of the blade cause a negative angle of attack on the outboard portions of the blade on the advancing side.

Figures 6.5(b) and 6.6(b) show the contours of the pitching moment at various forward flight speeds for upper and lower rotors. There is an increase in the negative pitching moment on the advancing side, roughly $\psi=45$ to $\psi=135$ degrees, on the outboard regions. On the retreating side, there is an increase in magnitude of the nose-up pitching moment and an increase in spanwise extent of this region, characteristic of the reverse flow region. At the two higher speeds, there is an isolated region of negative pitching moment, in the third quadrant, which may be indicative of dynamic stall. On the aft of the rotor disk, $\psi=0$ degrees, increase in positive pitching moment can be observed close to the tip of the blade due to the blade vortex interactions. The magnitude of the pitching moment due to the $8 \mathrm{P}$ 
blade passage also increases with forward flight speed.

Figure 6.5(c) and 6.6(c) show the chordwise force distribution on the upper rotor for a range of forward flight speeds. The distribution of the chordwise force on the rotor disk significantly changes as a function of forward flight speeds. At low speeds, 55 and 100 knots, the positive chordwise force is observed over the majority of the rotor disk, with coon the outer portions of the blade on the front half of the disk, $\psi=90$ to $\psi=270$ degrees. The contour levels show that the highest positive chordwise force is located on the outboard stations of the retreating blade, $\psi=225$ to $\psi=270$ degrees, at 55 knots. At 225 knots, the contour levels show that the highest positive chordwise force is observed on the midspan at $\psi=90$ degrees. Relatively low chordwise force is observed inboard at the front of the disk. With an increase in forward flight speed, the distribution of the positive chordwise force in this region moves inward.

The sectional normal force, shown in Fig 6.7, and the pitching moment, shown in Fig 6.8, show the vortex induced loading at low speeds as well as the dynamic stall phenomena at the highest speed. At the lowest flight speed, the interactions between the blades and the trailing vortices from the previous blades result in vortex induced loading at in the third quadrants, shown in Fig.6.8(b).

\subsection{Coaxial Rotor Interactional Aerodynamics - 50 Knots}

The objective of this section is to examine the interactional aerodynamics between the upper and the lower rotors of a coaxial rotor system. In previous 
section it was observed that at the lowest speed, 50 knots, there was a substantial difference in the upper and the lower collectives. The difference in the collectives was deemed to be a result of the interactional aerodynamics. This theory was tested out by running an additional CFD/CSD case, isolating upper and lower rotor in CFD but re-coupling forces from both rotors in CSD. This additional case isolated the interactional effects and showed effect of rotor-rotor interactions on the final trim solution. This latter case was initialized with the same initial CSD trim solution as in the coaxial CFD/CSD coupling 50 knot case discussed in previous section, where aerodynamics depend purely on the low order aerodynamic model and no CFD/CSD coupling has been performed.

Figure 6.9 shows the upper and the lower rotor normal force distribution, utilizing initial CSD deflections, for interactional (Case 1) and non-interactional (Case 2) cases. Take note that the CFD/CSD coupling procedure has not been performed yet and the objective of analyzing this set of airloads is to understand the effect of the interactional aerodynamics on the airloads. Also, the deflections for the upper and the lower rotor, for initial CSD, are almost identical. For the given deflections, there is a significant loss in normal force produced in the 3rd quadrant for the lower rotor, when the lower rotor is operating below the upper rotor. The induced velocity from the upper rotor wake decreases the sectional angle of attack, reducing the lift on the lower rotor. The lower rotor does not produce the same amount of normal force as the upper rotor at the given collective. Therefore in order for the lower rotor to produce the required normal force it needs to operate with higher collective. The isolated lower rotor, operating with the same deflection 


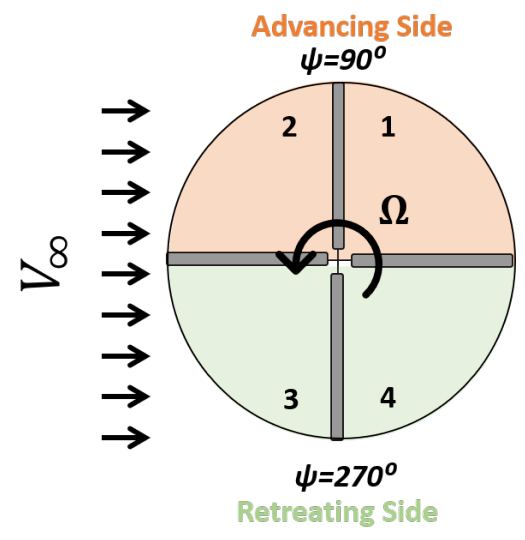

(a) Upper rotor

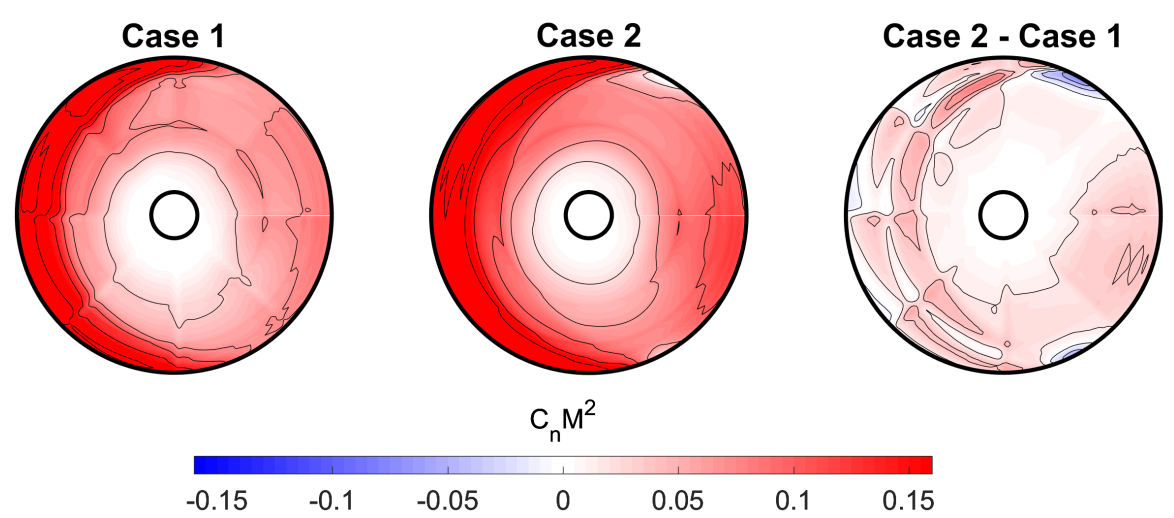

(c) Upper rotor

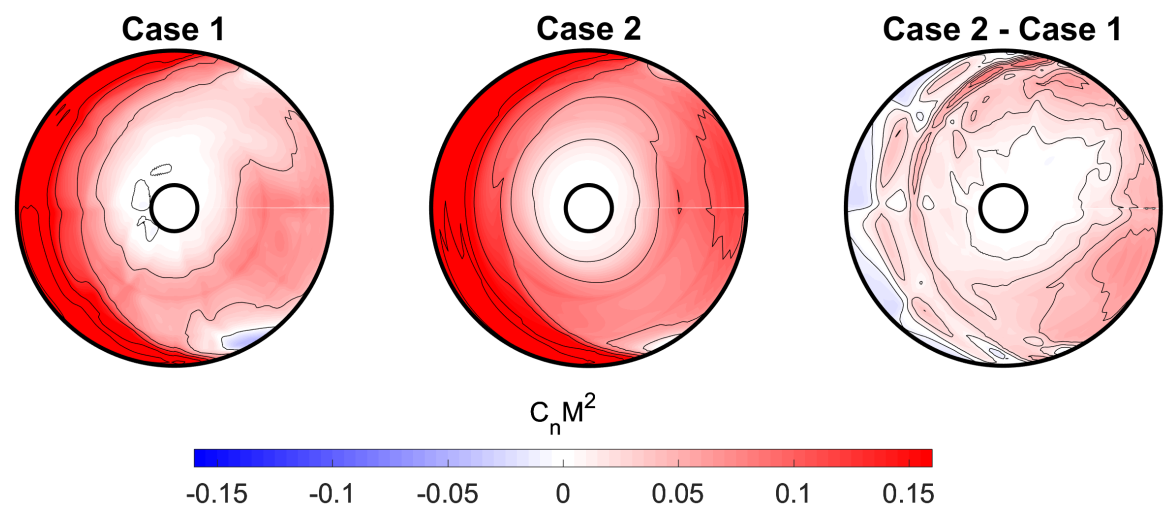

(d) Lower rotor

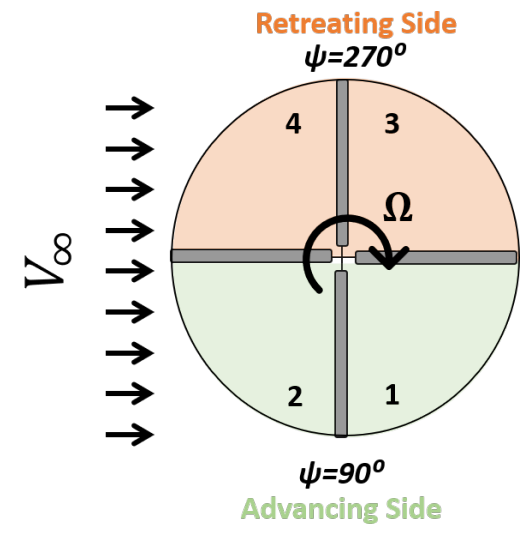

(b) Lower rotor 
as the lower rotor in the coaxial rotor system does not see the extreme drop in lift in the first quadrant. The lower rotor and the upper rotors, in isolation, produce higher normal force compared to the rotors operating in the coaxial rotor system. The difference in the normal force for the rotors operating in isolated and coaxial configuration foreshadows the difference in the final trim state of the vehicle at these two different configurations.

Table 6.3 shows the initial and the converged coaxial CFD/CSD control angles and the vehicle pitch attitude at 50 knots for the two cases. The interactional case (Case 1) results in the upper and the lower rotor being trimmed to different final collectives, whereas, the non-interacting case (Case 2) results in the same collective for the upper the lower rotors. The final upper and the lower rotor, for Case 1, are trimmed to a higher collective than the initial CSD collectives, while the rotors in Case 2 were trimmed to lower collectives than the initial CSD. It was observed, in Fig.6.9(c) and (d), that the normal force for the upper and the lower rotor in Case 2 was very similar. Thus, it is of no surprise that without any interactions the upper and the lower rotor were trimmed to similar values. It was also observed that in the interactional case the lower rotor produced less normal force than the upper rotor. Therefore the final collective of the lower rotor is slightly higher than that of the upper rotor, for the interactional case. The difference plot showed that the rotors in isolation produced more normal force than those in the coaxial configuration, which explains the change in the final collectives for both cases compared to the initial predictions.

For both cases, the rotors were trimmed to a higher final cyclic pitch $\theta_{1 c}$ 
Table 6.3: The final coaxial CFD/CSD controls and vehicle pitch attitude at 50 knots with interactional effects (Case 1) and without interactional effects (Case 2).

\begin{tabular}{l|ll|ll|ll|l}
\hline \hline & $\theta_{0}^{C C W}$ & $\theta_{0}^{C W}$ & $\theta_{1 c}^{C C W}$ & $\theta_{1 c}^{C W}$ & $\theta_{1 s}^{C C W}$ & $\theta_{1 s}^{C W}$ & $\alpha_{F}$ \\
\hline \hline Initial & 8.12 & 8.12 & 0.25 & 0.25 & -1.99 & 1.99 & -0.38 \\
\hline Case 1 & 9.58 & 9.86 & 3.61 & 3.61 & -3.15 & 2.70 & -0.42 \\
\hline Case 2 & 7.46 & 7.46 & 2.48 & 2.48 & -1.81 & 1.74 & -0.68 \\
\hline \hline
\end{tabular}

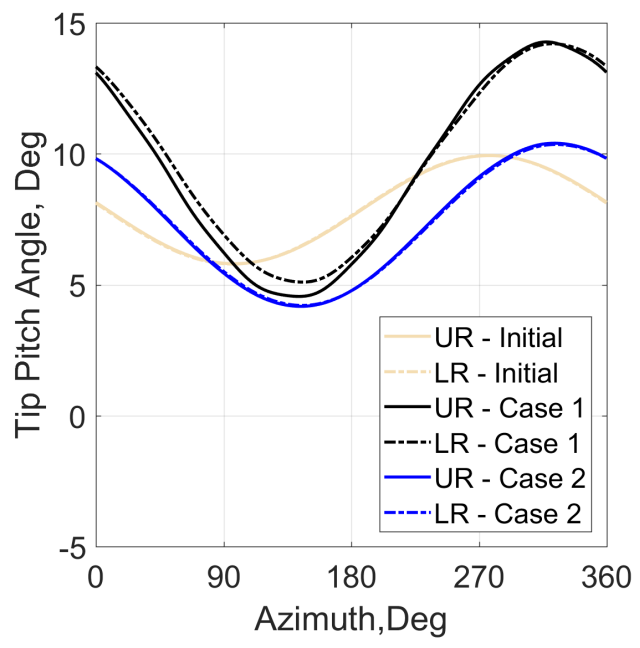

(a)

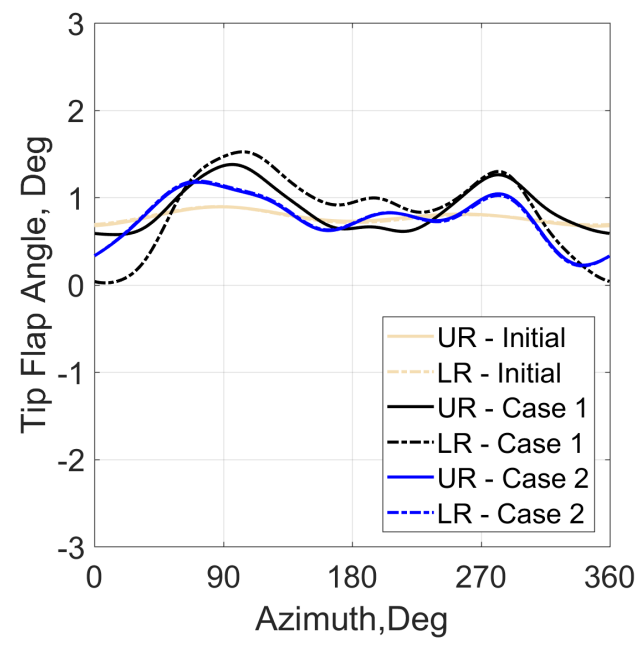

(b)

Figure 6.10: The upper and the lower rotor tip pitch and flap deflections for interactional and non-interacting cases at 50 knots. 
compared to the initial prediction. The tip pitch deflection is shown in Fig6.10(a). The initial CSD prediction trimmed the rotors to very low cyclic pitch $\theta_{1 c}$ as shown in Table 6.3. The low $\theta_{1 c}$ resulted in similar blade pitch on the fore and the aft of the rotor disk which produced a significant imbalance of normal force on the rotor disk, especially for the lower rotor in Case 1 . Thus, the final cyclic pitch $\theta_{1 c}$ was increased to lower the blade pitch over the nose of the aircraft, where high normal force concentration was observed, and increase the blade pitch over the tail.

Figure 6.11 shows the contour plots of the normal force distribution, on the upper and the lower rotors, for the final CFD/CSD iteration of the interactional and non-interactional case at 50 knots. The CFD/CSD coupling procedure was performed until the trim solution did not vary significantly. The difference between Case 2 and Case 1 normal force is also shown in Fig. 6.11. The sectional normal force at two radial locations, $45 \% R$ and $98 \% R$, is shown in Fig. 6.12 (a) and (b). The non-interactional case, Case 2, shows the upper and the lower rotor to have almost identical normal force distribution, which is a result of similar blade pitch distribution. The difference plots, for the upper and the lower rotors, show that Case 1 has higher normal force distribution on the outboard stations, at the front of the rotor disk. In these regions of the rotor disk, the Case 1 blade pitch is higher than for Case 2. It can also be said that the tip vortices are stronger for Case 1. In both cases, upper and lower rotor have very high normal force on outboard sections of the blades in 2nd and 3rd quadrants. Blade vortex interactions are responsible for the high normal force. The tip vortices from previous blades are staggered above 
the blade at 180 degrees from the retreating blade is traveling above the blade in these quadrants.

For the upper rotor and the lower rotor, the non-interactional case shows a loss in normal force in the first quadrant and third quadrants near the tip of the blade as shown in Fig 6.12(b). The upper and the lower rotor in the interactional case also experience a drop in the normal force in the first and third quadrants. This drop in the normal force in the first and the third quadrants are due to the tip of the blade passing through the coalescence of the tip vortices from previous blades. Figures 6.13 and 6.14 show the pitching moment contour plots for the upper and the lower rotors, for interactional and non-interactional cases. The interactional case exhibits strong $8 \mathrm{P}$ impulses in the pitching moment every 45 degrees which are absent from the non-interactional case. The impulses are due to the blade crossings. The $8 \mathrm{P}$ impulses are stronger for the upper rotor in the interactional case. 


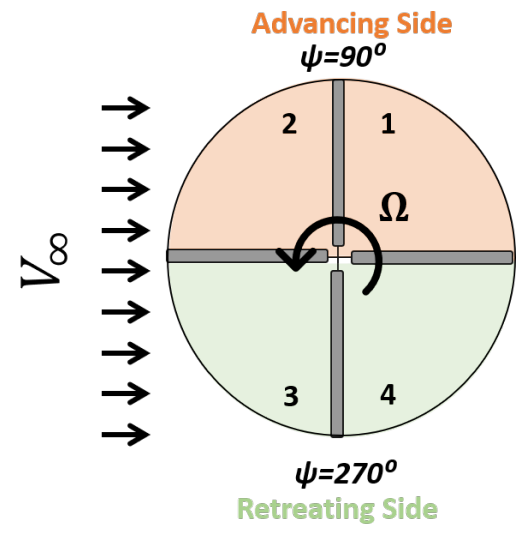

(a) Upper rotor

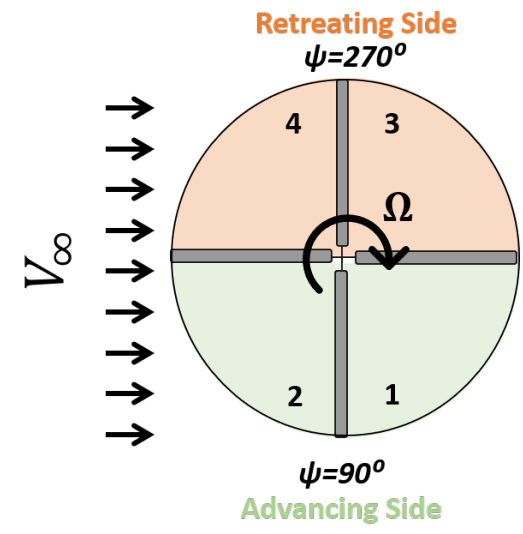

(b) Lower rotor

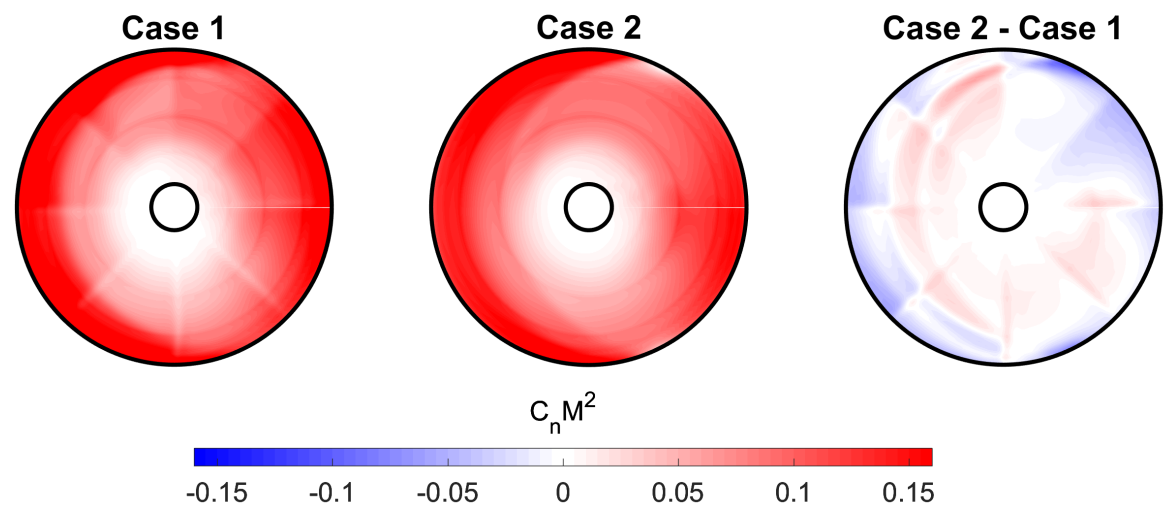

(c) Upper rotor

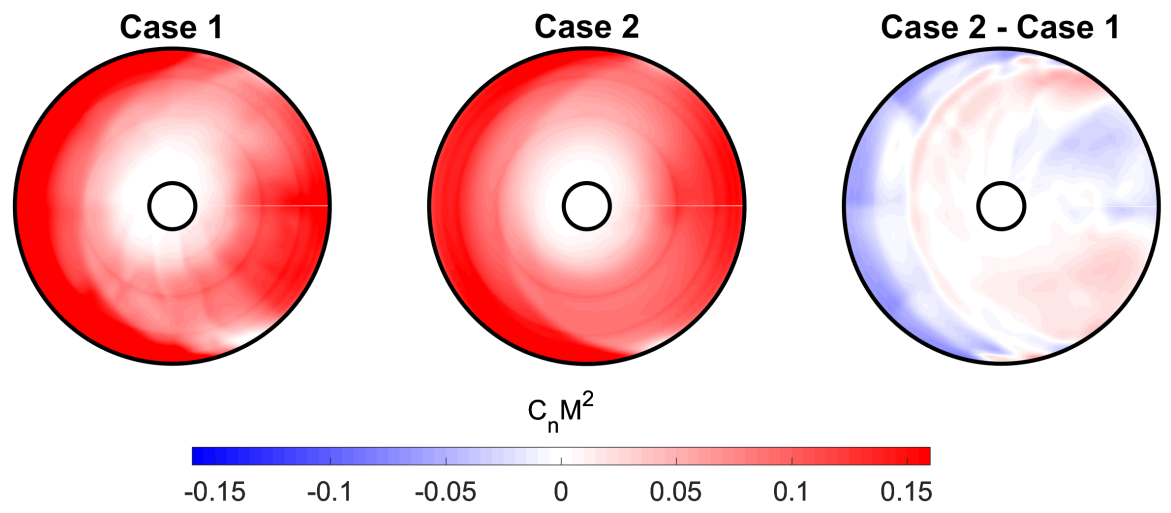

(d) Lower rotor

Figure 6.11: The upper (a) and lower (b) rotor normal force at 50 knots for interactional and non-interactional case at final CFD/CSD coupling iteration. 

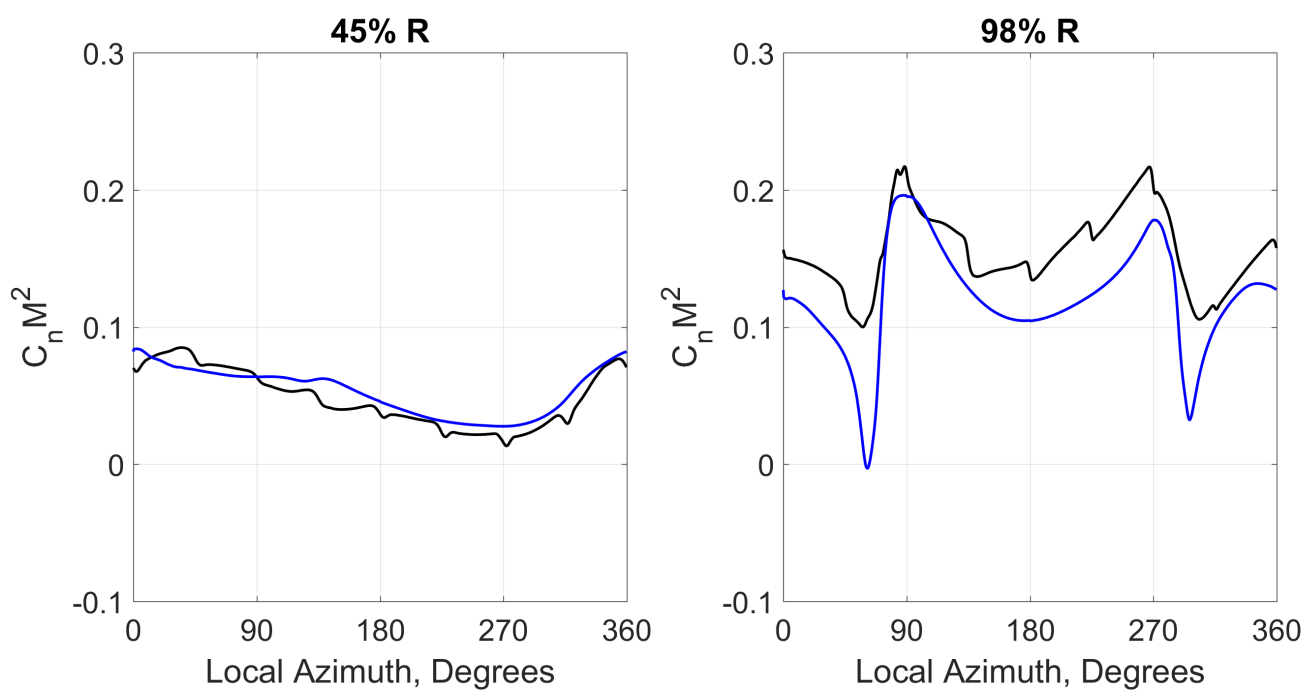

(a) Upper Rotor
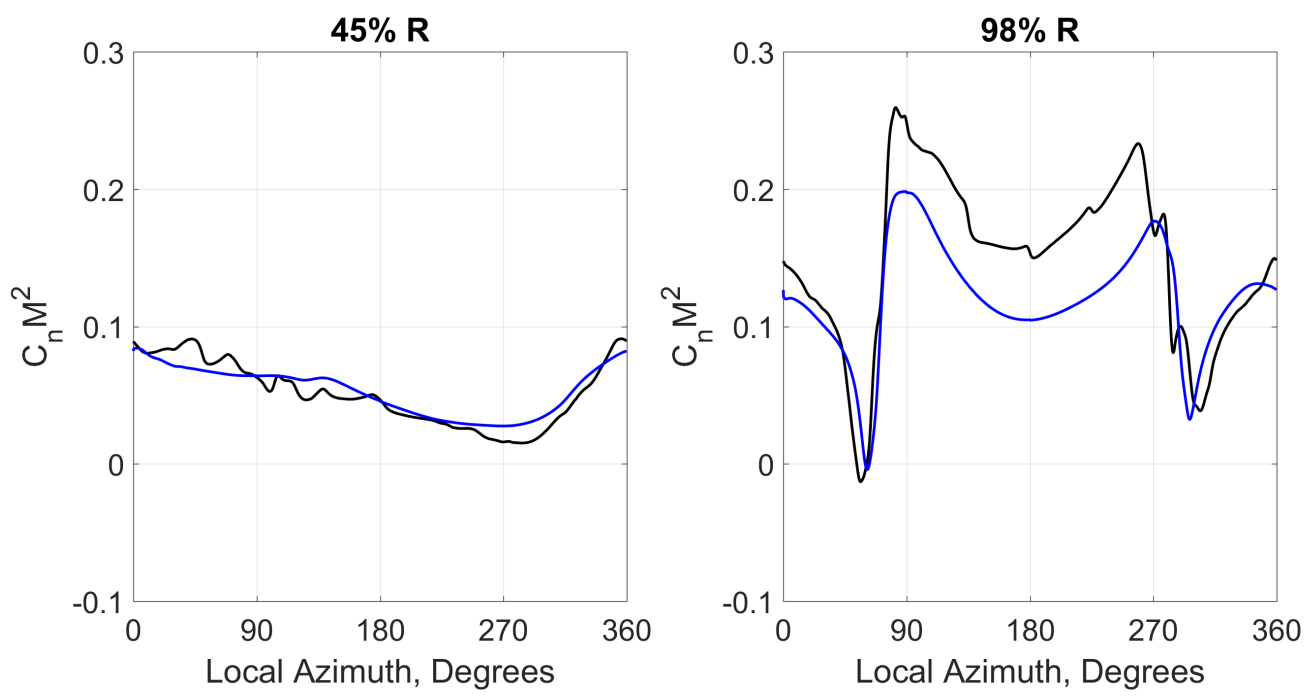

(b) Lower Rotor

Figure 6.12: The upper (a) and lower (b) rotor sectional normal force at 50 knots for interactional and non-interactional case. 


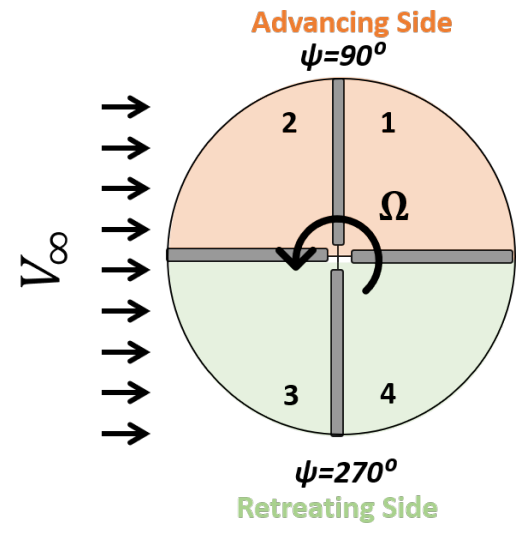

(a) Upper rotor
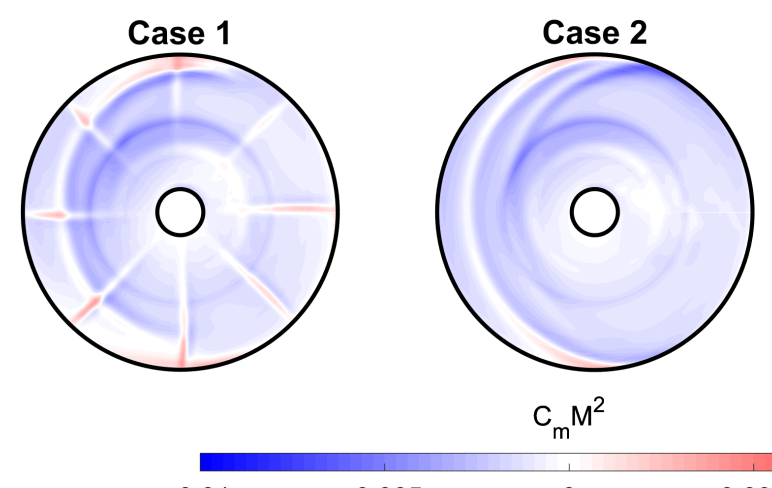

(c) Upper rotor

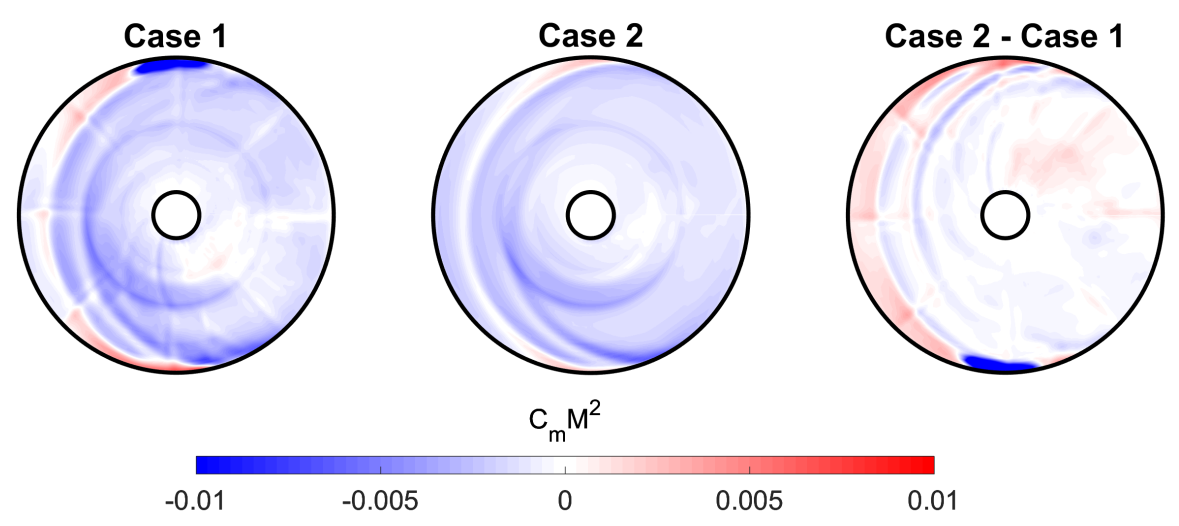

(d) Lower rotor

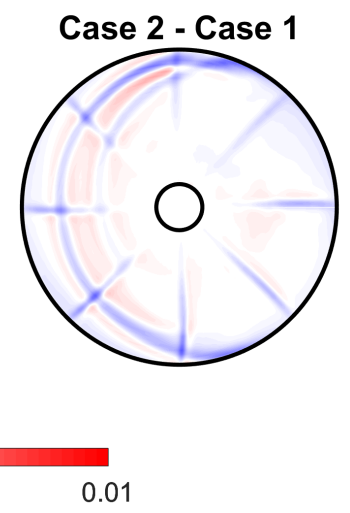

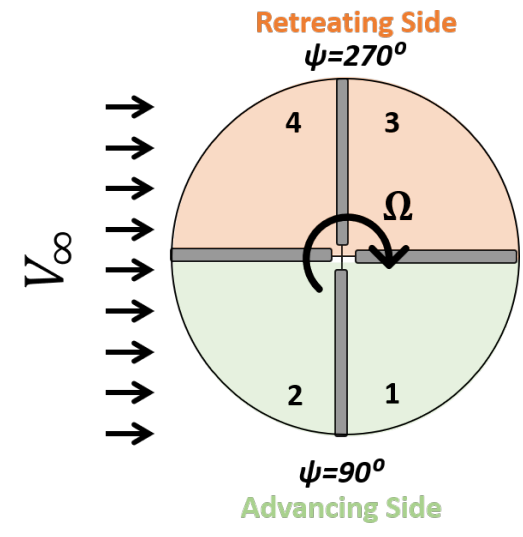

(b) Lower rotor 

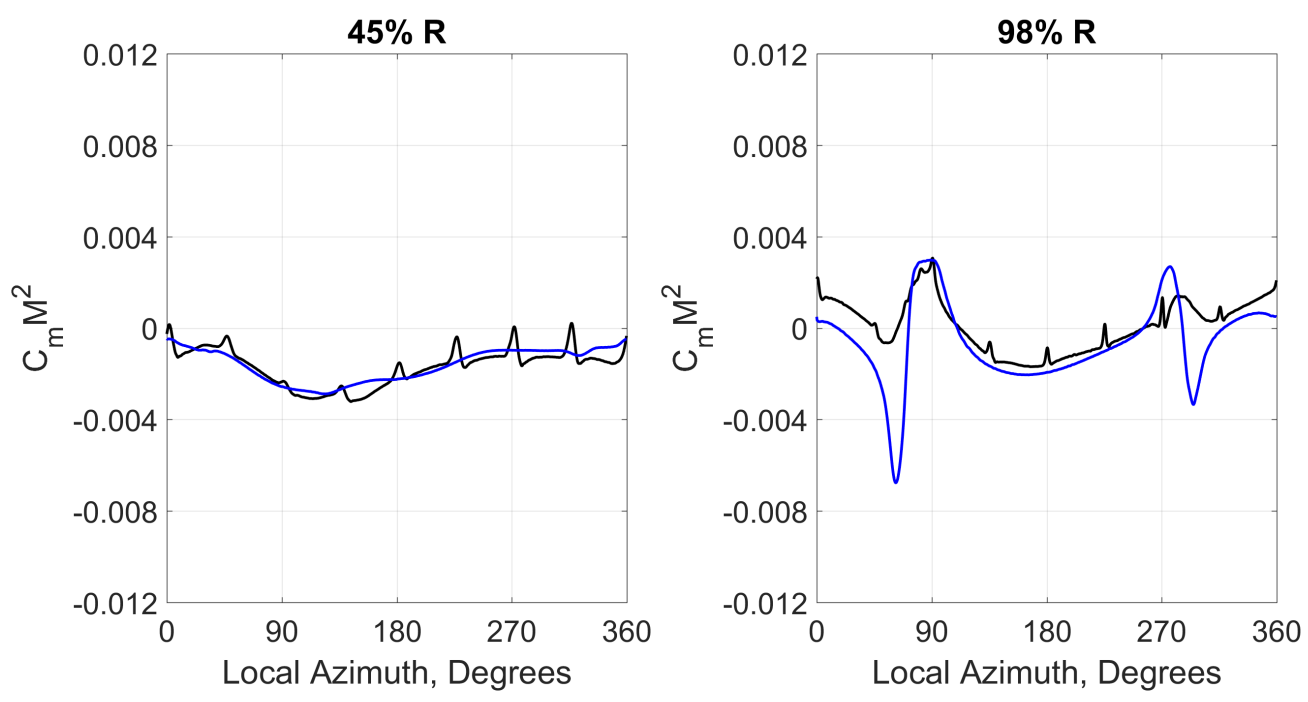

(a) Upper Rotor
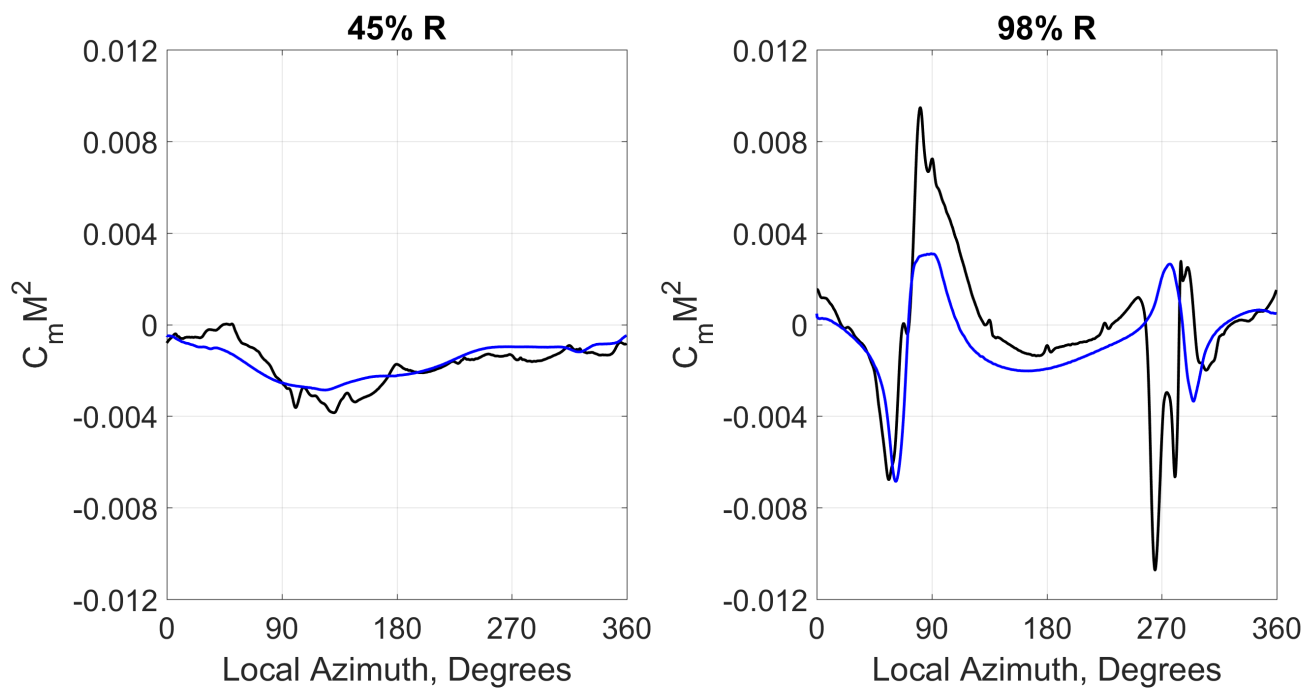

(b) Lower Rotor

Figure 6.14: The upper (a) and lower (b) rotor sectional normal force at 50 knots for interactional and non-interactional case. 


\section{Results Part II: Vehicle CFD/CSD Coupling}

This chapter examines the effect of the full vehicle CFD/CSD coupling on the final trim parameters, elastic blade deflections, aerodynamic and structural loads. The results from the full vehicle CFD/CSD coupling and the coaxial rotor CFD/CSD coupling are compared. Some of the results described in the following chapter were briefly discussed in Ref. [85. The full vehicle CFD/CSD coupling simulations are more expensive than the coaxial rotor CFD/CSD coupling simulations; an addition of the unstructured helicopter airframe and unstructured propeller to the CFD increases the CFD computation time by a factor of five. Thus, it is important to demonstrate the effects that the full vehicle CFD/CSD coupling has on the final trim state of the vehicle. Each vehicle CSD iteration involves a auxiliary propeller wind tunnel trim and a separate full vehicle trim. There are various differences between the isolated coaxial CFD/CSD trim procedure and a full helicopter configuration procedure:

1. The addition of the airframe and the auxiliary propeller in the CFD simulations.

2. The input of the time-averaged airframe loads and time-averaged auxiliary propeller power from the CFD into the comprehensive analysis. 
3. The auxiliary propeller is trimmed for the target power obtained from the literature [6], while accounting for the installation effects.

The results in this section are shown for a forward flight speed of 150 knots, with case parameters given in Table 7.1 .

Table 7.1: CFD interactional aerodynamics case

\begin{tabular}{ccccccc}
\hline \hline$V_{\infty}($ knots $)$ & $\mu$ & MR RPM & $M_{\text {adv.tip }}$ & $\operatorname{LOS}(\%)$ & Blade Modes & Harmonics \\
\hline \multirow{2}{*}{150} & 0.41 & 446 & 0.79 & 11.3 & 6 & 8 \\
\hline \hline
\end{tabular}

\subsection{Trim Parameters}

This section examines the effects of the full vehicle CFD/CSD coupling on the control angles of the coaxial rotor system and the attitude of the airframe, as well as the propeller collective. The rotor deflections from an isolated coaxial CFD/CSD simulations were used as a starting point for the full vehicle CFD/CSD coupling process. However, it is not necessary to start the full vehicle CFD/CSD coupling process from an isolated coaxial rotor cases. Nine coupling iterations were performed for the full CFD/CSD coupling case, ensuring that the trim parameters did not vary significantly with consecutive iterations.

Understanding of the mechanisms responsible for the differences between the final trim controls, of the coaxial CFD/CSD and the full vehicle CFD/CSD, can be achieved by analyzing the force and moment equilibrium of the vehicle. For the vehicle to be in trim, the force and moment equilibrium must be enforced at $\mathrm{CG}$, 
for every rotor revolution. The force and moment contributions are closely tied to the rotor trim controls and the vehicle attitude. Thus, this subsection will focus on relating the changes in the vehicle forces/moments to the main rotor controls and airframe attitude. The forces and moments described in this section are along an axis where $x_{C G}$ points towards the tail, the $y_{C G}$ points starboard, and the $z_{C G}$ points upward. The center of this axis lies at the CG of the aircraft.

Figure 7.1 shows the convergence of main rotor control angles and the fuselage pitch attitude over various trim iterations (CFD/CSD coupling cycles) at 150 knots. The trim iterations 0 through 4, shown in the green section of Fig. 7.1, represent the coaxial rotor system CFD/CSD coupling procedure. The trim iterations 5 through 13, shown in the yellow section of Fig. 7.1, represent the full vehicle CFD/CSD coupling. There is a difference between the two cases, predominantly due to the interactional aerodynamic effects on the airframe and coupling of the airframe airloads from CFD into CSD.

Figure 7.1 (a) shows the convergence of the collective angle for the upper and the lower rotors, for coaxial rotor system alone as well as for vehicle CFD/CSD coupling. The collective angles for the upper and the lower rotor, from the coaxial CFD/CSD coupling, trimmed to similar values. After the inclusion of the airframe and the auxiliary propeller in the CFD simulations (trim iteration 5 and greater), the upper rotor collective was reduced while the lower rotor collective was increased. The differential in the collective can be explained from comparing the airframe yaw moments at CG from 4th and 13th trim iterations. Figures 7.2 (d-f) show the airframe aerodynamic roll, pitch and yaw moments at CG at 4th and 13th trim 
Table 7.2: Comparison between final coaxial CFD/CSD and full vehicle CFD/CSD

\begin{tabular}{c|l|l}
\hline \multicolumn{1}{c|}{ 150 Knots } & Coax CFD/CSD & Full Vehicle CFD/CSD \\
\hline \hline Collective (deg) & $6.02(\mathrm{CCW})$ & $5.76(\mathrm{CCW})$ \\
& $5.95(\mathrm{CW})$ & $7.04(\mathrm{CW})$ \\
\hline Cyclic $\theta_{\mathbf{1 C}}(\mathbf{d e g})$ & $0.96(\mathrm{CCW})$ & $2.43(\mathrm{CCW})$ \\
\hline Cyclic $\theta_{\mathbf{1 S}}(\mathbf{d e g})$ & $0.96(\mathrm{CW})$ & $2.43(\mathrm{CW})$ \\
\hline Fuselage Pitch $(\mathbf{d e g})$ & 2.01 & $-5.63(\mathrm{CCW})$ \\
\hline
\end{tabular}




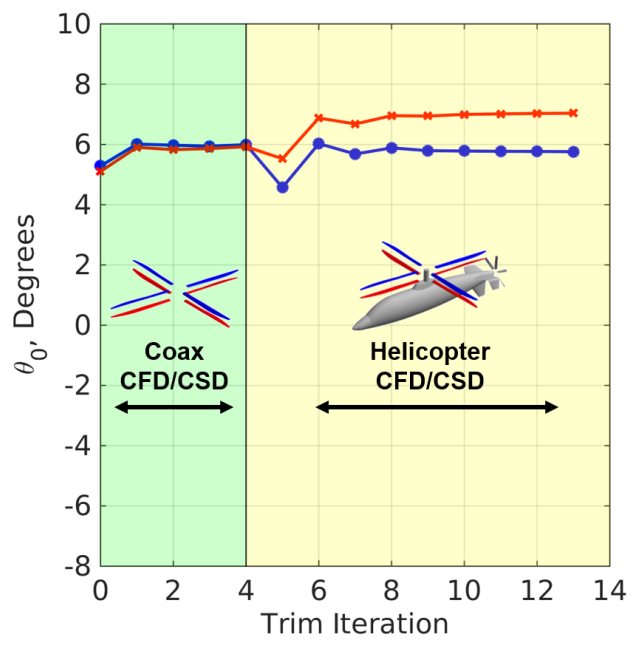

(a)

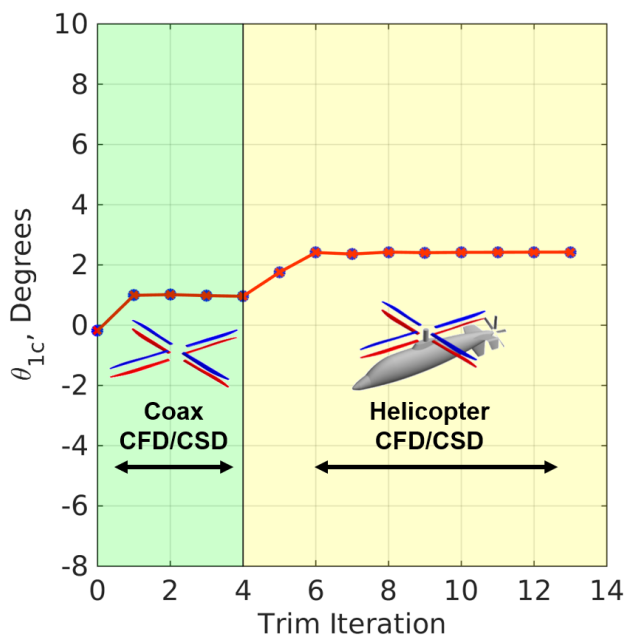

(c)

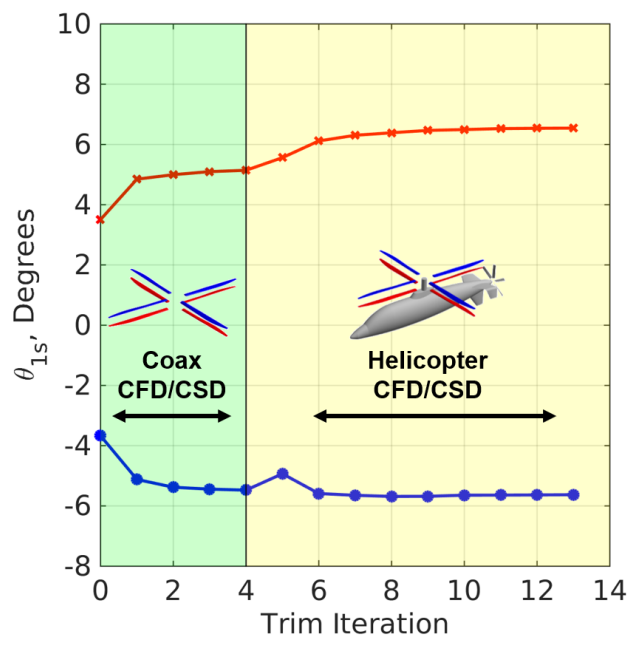

(b)

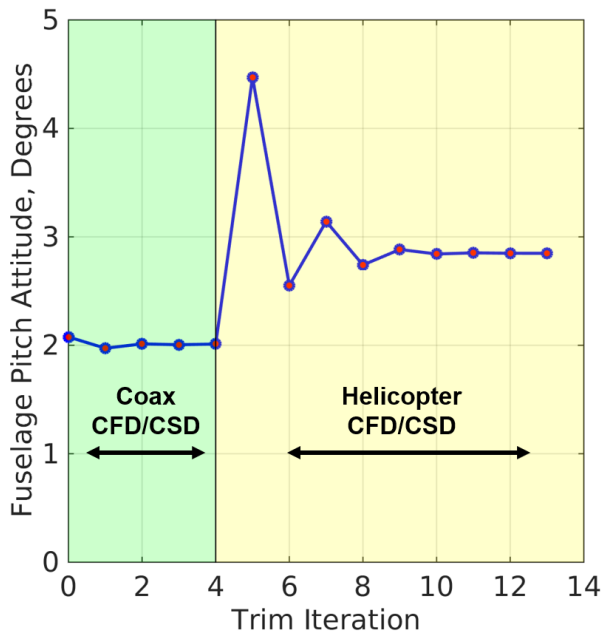

(d)

Figure 7.1: Rotor controls and fuselage attitude convergence history for coax and full vehicle CFD/CSD coupling at 150 knots. 


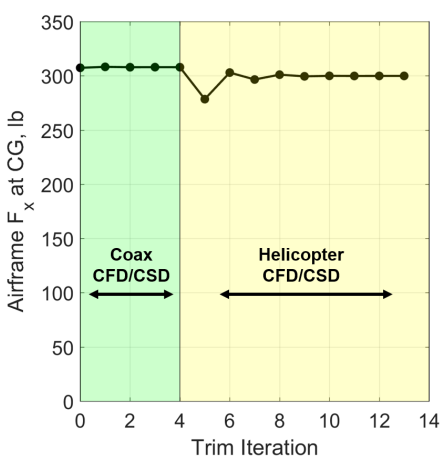

(a)

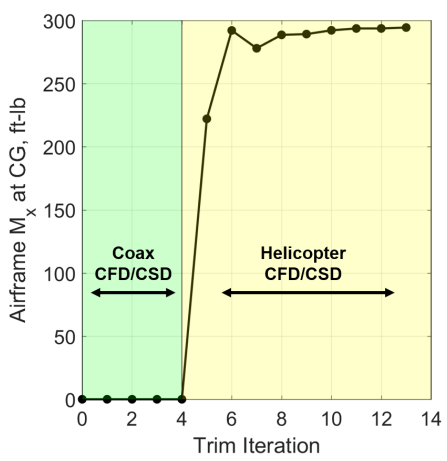

(d)

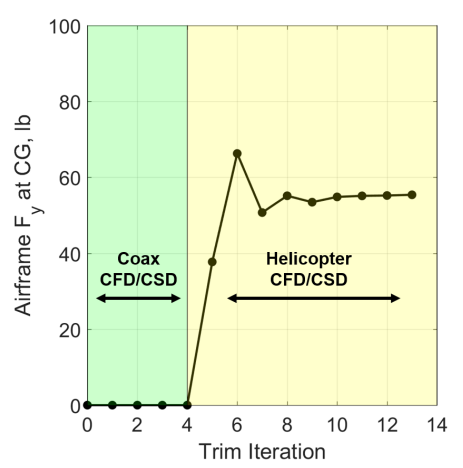

(b)

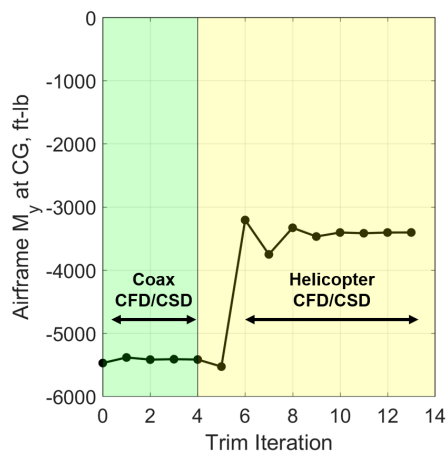

(e)

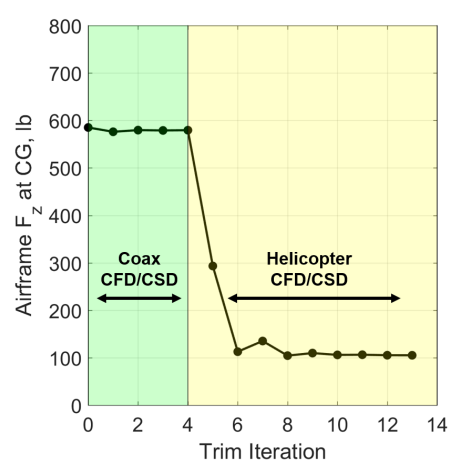

(c)

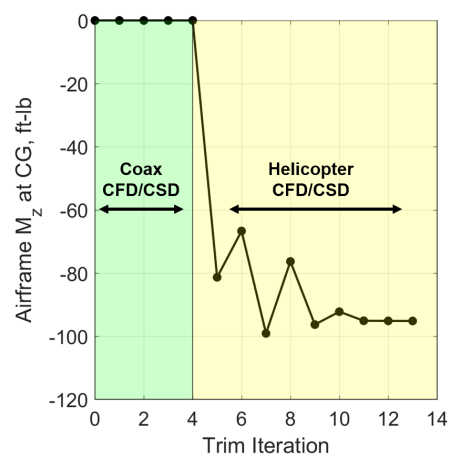

(f)

Figure 7.2: Convergence of airframe loads at CG at 150 knots.

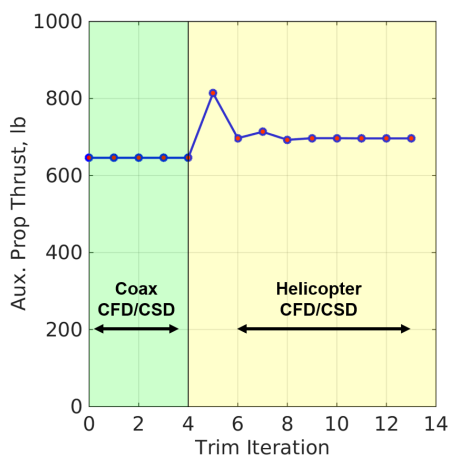

Figure 7.3: Pusher propeller thrust convergence at 150 knots. 
the clockwise moment from the airframe.

Figure $7.1(\mathrm{~b})$ compares the convergence of the $\theta_{1 s}$ control angle between the coaxial and the full vehicle CFD/CSD coupling. There is a difference between the final trimmed coaxial CFD/CSD and the final full vehicle CFD/CSD $\theta_{1 s}$ control angle, for both the upper and the lower rotor. For the final full vehicle CFD/CSD coupling, the lower rotor $\theta_{1 s}$ trimmed to a higher value than the upper rotor, providing excessive roll moment starboard side down. This can be explained by analyzing the difference in the roll moment on the airframe for the two different coupling methodologies. There is also a negative aerodynamic roll moment, port side down, at CG produced by the airframe, shown in Fig.7.2(d). In order for the system to be in trim, the roll moment from the airframe needs to be compensated by the differential roll moment from the rotors.

Figure 7.1 (c) shows that there is an increase in the $\theta_{1 c}$ control angle from trim iteration 4 (coaxial CFD/CSD) to trim iteration 13 (full vehicle CFD/CSD). The increase in the $\theta_{1 c}$ control angle can be explained by analyzing the pitching moment produced by the airframe. Figure 7.2 (e) shows an increase in the nose-down airframe pitching moment. The pitching moment produced by the rotors needs to increase to counteract the nose down pitching moment produced by the airframe, resulting in an increase of the $\theta_{1 c}$.

The axial body force, $F_{x}$, equilibrium is related to the airframe pitch attitude through the balance of the helicopter drag and the propeller thrust. The axial force from the coaxial rotor system and the airframe must be counteracted by the auxiliary propeller. The coaxial CFD/CSD coupling procedure assumes an approximation for 
the prop thrust, calculated from a simple relation, $P=T V$, where $P$ is the propeller power for the X2TD given in Ref. [46]. Whereas, in the full vehicle CFD/CSD coupling procedure, the propeller thrust is obtained from the propeller CFD airloads for the propeller trimmed to the required power given in Ref. [46]. Figure 7.1(b) shows an increase in the rearward tilt of the rotor disk, between trim iteration 4 and 13. The rotor disk is tilted rearward to counteract an increase in the propeller thrust, shown in Fig. 7.3 .

\subsection{Main Rotor Blade Pitch and Flap}

This section examines the effects of the full vehicle CFD/CSD coupling on the upper and the lower rotor blade tip pitch and flap deflections. The pitch shown in this section is a combination of the control pitch and the elastic twist, it does not take into account the blade geometric twist angle.

Figure 7.4 shows the tip pitch angle of the main rotor blade, as the blade travels around the azimuth. Take note that the azimuth is shown in the local rotor reference frame where a positive pitch angle represents a pitch-up motion around the quarter chord line while a negative pitch angle represents a pitch-down motion around the quarter chord line. Figure 7.4(a) compares the upper rotor tip pitch obtained from the final coaxial CFD/CSD trim (Trim 4) and the final full vehicle CFD/CSD trim (Trim 13). The nomenclature for trim iterations is consistent with the one shown in the previous section. A $1 \mathrm{P}$ pitch variation is observed for both coaxial and full vehicle CFD/CSD coupling cases. 
Table 7.3: Comparison between final coaxial CFD/CSD and full vehicle CFD/CSD pitch deflection coupling at 150 knots.

\begin{tabular}{c|l|l}
\hline \hline \multicolumn{1}{c|}{$\mathbf{1 5 0}$ Knots } & Coax CFD/CSD & Full Vehicle CFD/CSD \\
\hline \hline Mean Pitch (deg) & $5.83(\mathrm{CCW})$ & $5.61(\mathrm{CCW})$ \\
& $5.76(\mathrm{CW})$ & $6.86(\mathrm{CW})$ \\
\hline 1P Pitch Amplitude (deg) & $5.72(\mathrm{CCW})$ & $6.31(\mathrm{CCW})$ \\
& $5.39(\mathrm{CW})$ & $7.15(\mathrm{CW})$ \\
\hline
\end{tabular}

The full vehicle CFD/CSD coupling approach resulted in a decrease of upper rotor mean tip pitch and an increase in 1P pitch amplitude, shown in Fig.7.4(a) and Table 7.3. The lower rotor experienced an increase in mean tip pitch, as well in an increase in 1P amplitude, shown in Table 7.3 and Fig.7.4(b). The lower rotor mean pitch increase and upper rotor mean pitch decrease is due to the need to counteract the additional yaw moment from the airframe, as described in previous section. An increase in the $1 \mathrm{P}$ variation reflects an increase in the roll moment needed to counteract the additional airframe roll moment.

The coaxial CFD/CSD coupling approach resulted in the upper and the lower rotors being trimmed to similar pitch deflections, with small differences on the advancing side near $\psi=90$ degrees and the retreating side near $\psi=270$ degrees, shown in Fig.7.6(a). The upper and the lower mean tip pitch angle, obtained with the coaxial CFD/CSD approach, shown in Table 7.2 , differed by roughly 0.05 degrees with less than 0.5 degree difference in the $1 \mathrm{P}$ amplitude. The small differences in 
the upper and lower pitch can be attributed to interactional aerodynamics between the rotors.

Figure 7.5(a) compares the upper rotor tip flap angle for the coaxial and the full vehicle CFD/CSD coupling methods. The coaxial CFD/CSD coupling approach trims the rotor to flap down over the vehicle tail and flap up over the nose. The full vehicle CFD/CSD coupling approach results in a nearly zero upper rotor blade flap over the tail of the vehicle, with small positive blade flap over the nose. Similarly for the lower rotor, the coaxial CFD/CSD coupling results in the blades flap down over the tail and flap up over the nose, as shown in Fig.7.5(b). However, the full vehicle CFD/CSD coupling approach results in a nearly zero lower rotor blade flap over the tail of the vehicle, with some positive blade flap over the nose. The flap over the nose produces a cumulative rotor hub pitching moment to counteract the pitching moment from the airframe and horizontal stabilizers. As discussed in the previous section and as shown in 7.2 (e), the pitching moment produced by the airframe was lower for full vehicle CFD/CSD coupling approach, therefore the rotors did not have to produce as much pitching moment as for the coaxial CFD/CSD coupling procedure.

Figure 7.6(b) shows the lower and the upper rotor tip flap deflection, from the coaxial and the full vehicle CFD/CSD approach, on the same plot. The coaxial CFD/CSD coupling approach results in the upper and the lower rotor having indistinguishable flap up deflection on the advancing side, $\psi=90$ degrees, as well as on the retreating side, $\psi=270$ degrees. The full vehicle CFD/CSD approach results in the lower rotor having larger flap angle than the upper rotor, on both the advancing 


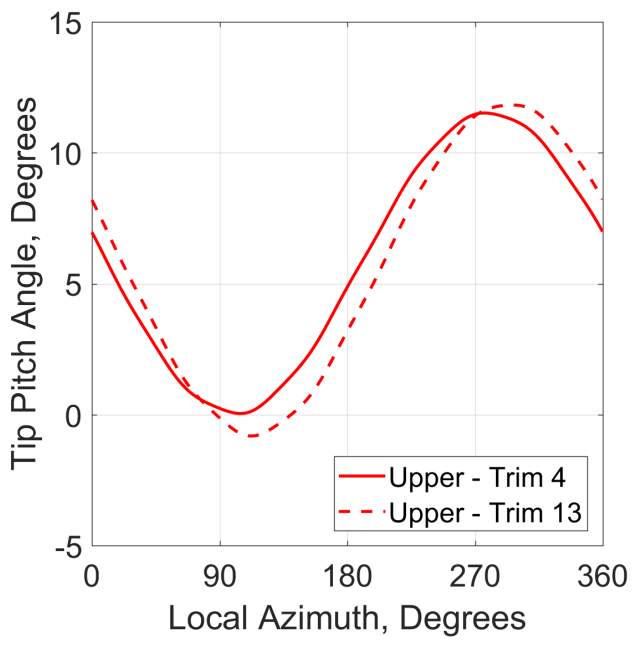

(a)

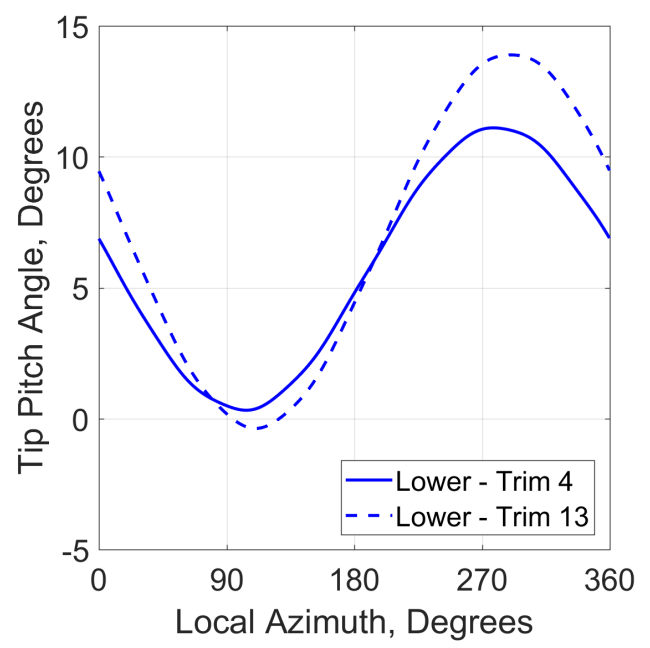

(b)

Figure 7.4: Upper rotor and lower rotor tip pitch deflections for coaxial and full vehicle CFD/CSD coupling at 150 knots.

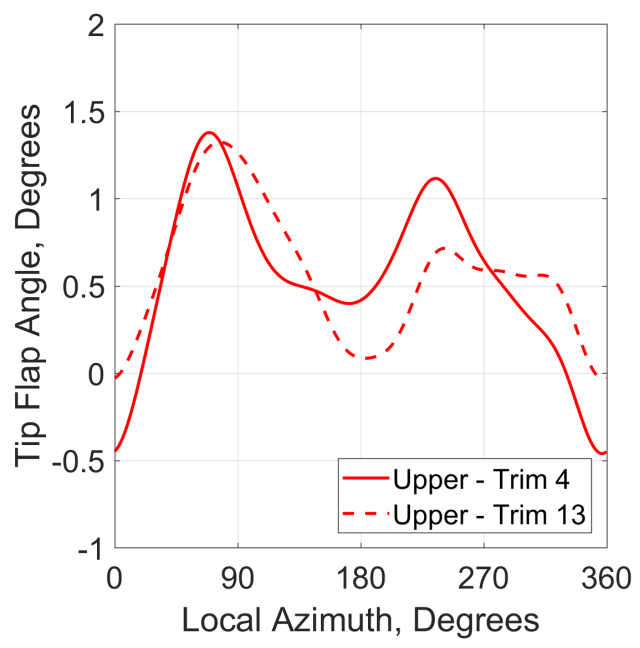

(a)

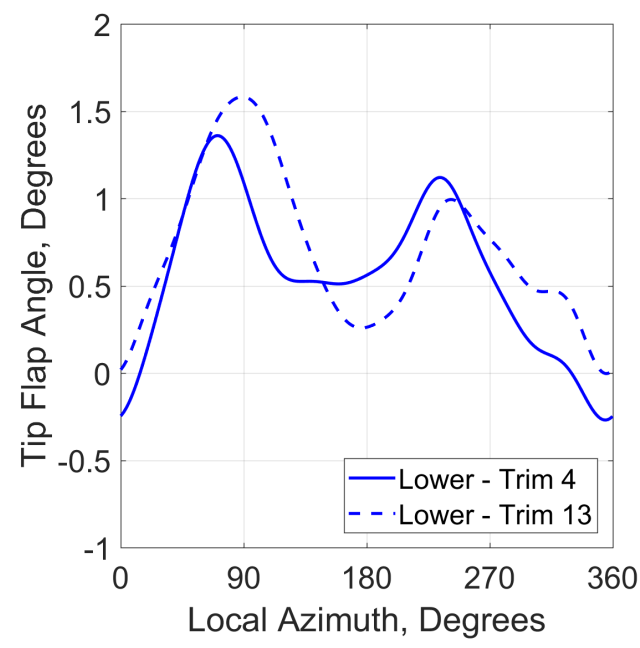

(b)

Figure 7.5: Upper rotor and lower rotor tip flap deflections for coaxial and full vehicle CFD/CSD coupling at 150 knots. 

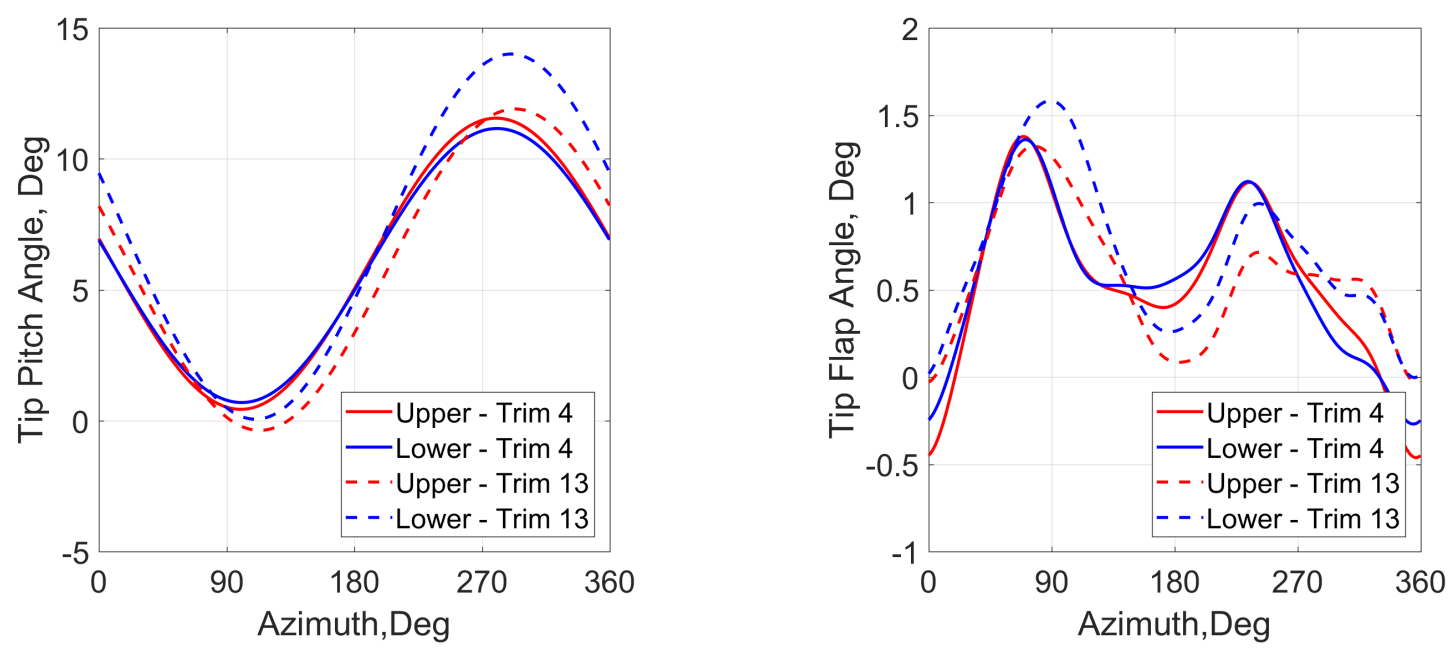

Figure 7.6: Upper and lower rotor tip flap and pitch deflections for coaxial and full vehicle CFD/CSD coupling at 150 knots.

and the retreating sides. The differential in flap angle for full vehicle CFD/CSD coupling approach stems from the need to balance the port roll moment produced by the airframe.

\subsection{Main Rotor Airloads}

This section examines the upper and lower rotor airloads at the final coaxial CFD/CSD coupling iteration and full vehicle CFD/CSD coupling iteration. The objective of the section is to identify and analyze the differences in the rotor airloads resulting from the presence of the airframe and the auxiliary propeller and CFD as well as in the CFD/CSD coupling exchange. 


\subsubsection{Normal Force - Upper and Lower Rotor}

This subsection examines the effects of full vehicle CFD/CSD coupling and the presence of the airframe/propeller in CFD simulations on the normal force produced by the upper and the lower rotor. The azimuth convention for the upper rotor is given in Fig.7.7(a), which shows a top view diagram of the upper rotor disk rotating counter-clockwise. Figure 7.7(b) shows the upper rotor non-dimensional normal force. The diagram labeled (1) in Fig.7.7(b) shows the normal force from the final coax CFD/CSD coupling iteration, where the CFD simulations included only the coaxial rotor system. The diagram labeled (2) in Fig 7.7 (b) shows the normal force from the full vehicle CFD simulations in which the rotor utilized the elastic blade deflections from the final coaxial CFD/CSD coupling iteration. Therefore, the only difference between the two data sets labeled (1) and (2) is the presence of the airframe and the auxiliary propeller in CFD. The diagram labeled (3) in Fig.7.7(b) shows the normal force from the final full vehicle CFD/CSD simulation. Figure 7.7(c) shows the difference between the normal force in datasets (1) through (3) shown in Fig 7.7(b).

The first diagram in Fig.7.7(c), labeled (2) - (1), is the difference in upper rotor normal airloads between the (1) coaxial CFD/CSD simulation and (2) full vehicle CFD simulation utilizing same deflections as (1). The difference plot shows that in the presence of the airframe in the CFD, the normal force increases on the outboard sections of the upper rotor, over the nose of the aircraft. There is an upwash over the nose of the aircraft, decreasing the $U_{p}$ velocity at blade section, increasing the 


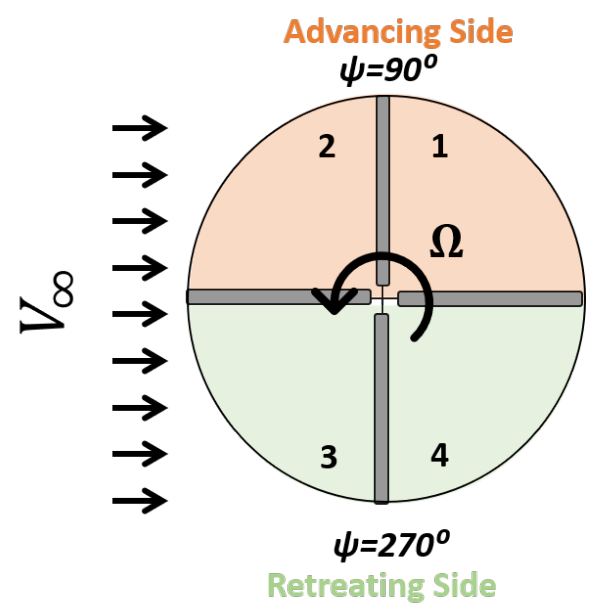

(a)

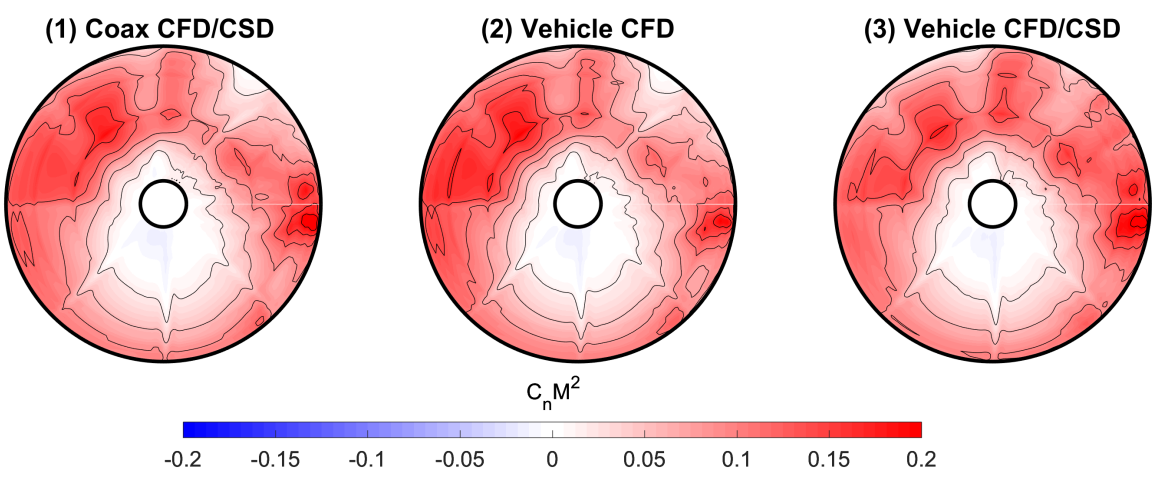

(b)

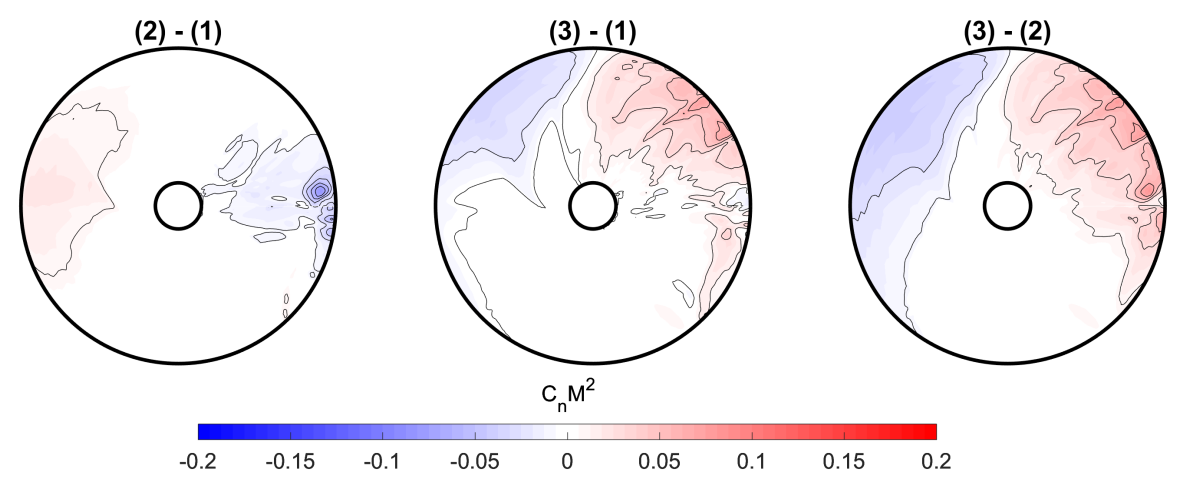

(c)

Figure 7.7: Non-dimensional normal force distribution for upper rotor, rotating counter-clockwise. 
sectional angle of attack on the blade over the nose. In the presence of the airframe, there is a decrease in rotor normal force over the tail due to the downwash produced by the airframe and the wake from the mast.

The second diagram in Fig.7.7(c), labeled (3) - (1), shows the difference between the upper rotor normal force obtained using the full vehicle CFD/CSD coupling approach and the coaxial CFD/CSD coupling approach, see plots (3) and (1) in Fig 7.7(b). The difference plot shows that the full vehicle CFD/CSD coupling trimmed the rotor to produce higher normal force in the first quadrant and lower normal force in the second quadrant. This can be correlated back to the blade pitch. Figure 7.4(a) showed that the blade tip pitch obtained with full vehicle CFD/CSD coupling was higher in the first quadrant and fourth quadrant but lower in the second and third quadrants compared to the coaxial CFD/CSD coupling.

The upper rotor non-dimensional sectional normal force at selected radial stations is shown in Fig. 7.8). The plot compares the sectional normal force for three datasets discussed above. The presence of the fuselage is observed over the nose of the aircraft (around 180 degrees), resulting in an increase of the sectional normal force and over the tail (around 0 degrees) resulting in the decrease of sectional normal force. The increase and decrease of normal force is more prominent on the outboard stations of the blades and on lower rotor. Again, the differences between the coaxial CFD/CSD coupling and the vehicle CFD/CSD coupling sectional normal force near the tip of the blade can be explained in terms of the blade tip pitch, Fig. 7.4(a).

Figure 7.9 (a) shows the azimuth convention for the lower rotor. Note the 

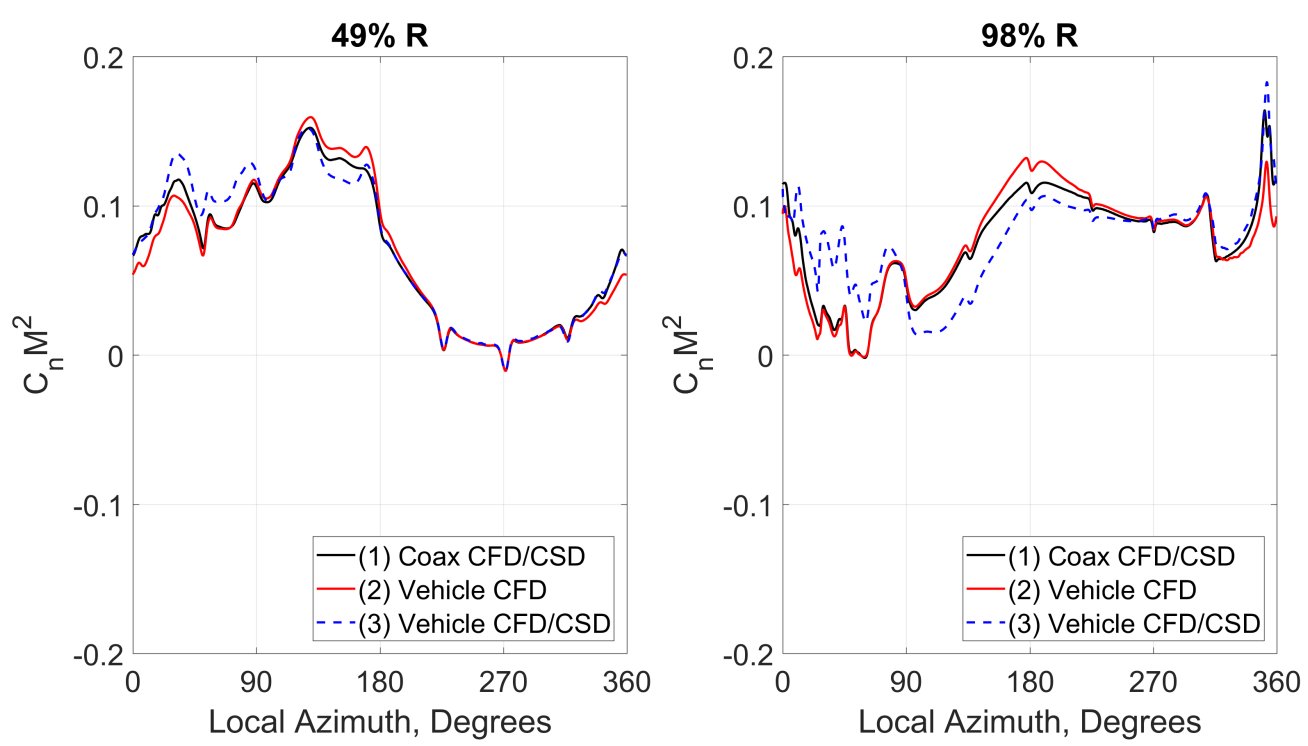

Figure 7.8: Comparison of upper rotor sectional normal force at 150 knots.

change in the location of the advancing and the retreating sides. Since the lower rotor is rotating clockwise, the advancing side of the lower rotor is on the port side of the aircraft.

Figure 7.9(b) compares the lower rotor normal force for three data sets: (1) final coaxial CFD/CSD coupling simulation, (2) vehicle CFD simulation utilizing the elastic blade deformations from final coaxial CFD/CSD simulation and (3) final full vehicle CFD/CSD coupling simulation. Figure 7.9(c) shows the difference between the three data sets, (1) through (3). The first diagram in Fig.7.9(c) shows the difference between dataset (2) and dataset (1). The presence of the airframe results in higher normal force on the fore portion of the rotor disk, over the nose of the aircraft, while the shedding from the mast and the downwash over the tail of the aircraft result in lower normal force on the aft portion of the rotor disk. The second diagram in Fig 7.9. (c) shows the difference between lower rotor normal force predicted 


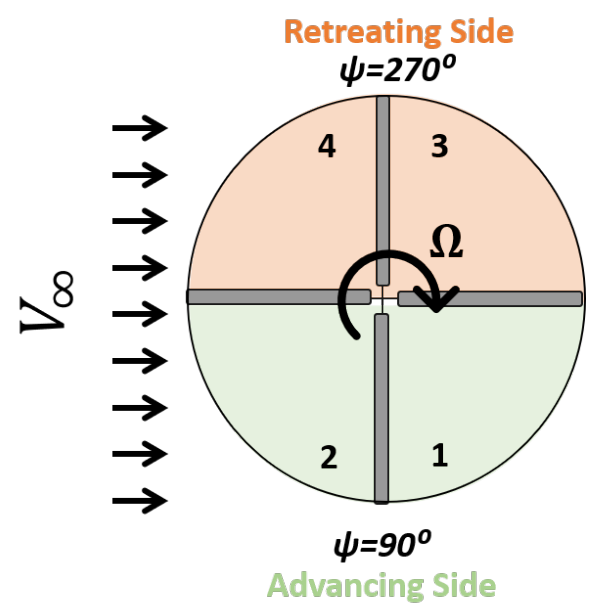

(a)

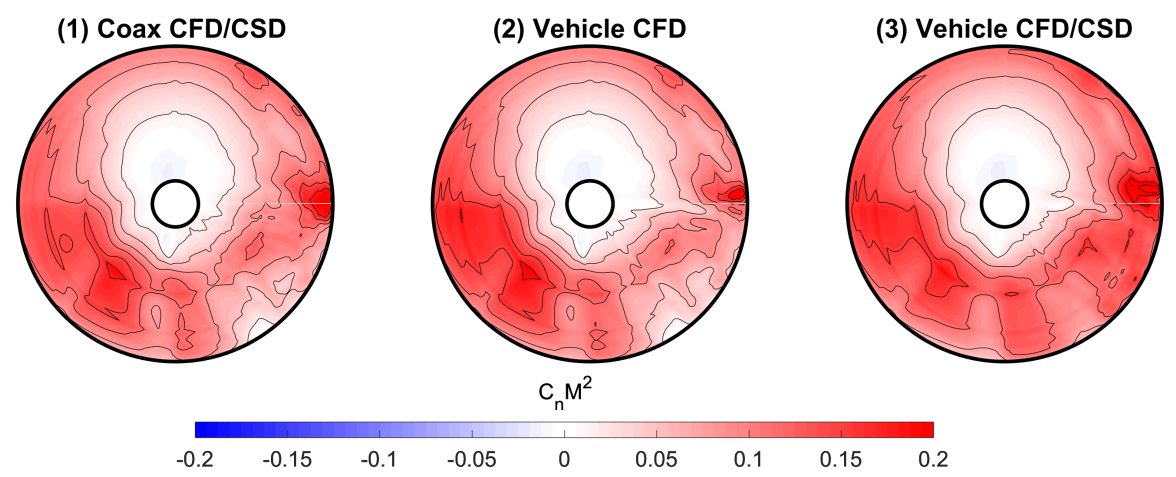

(b)

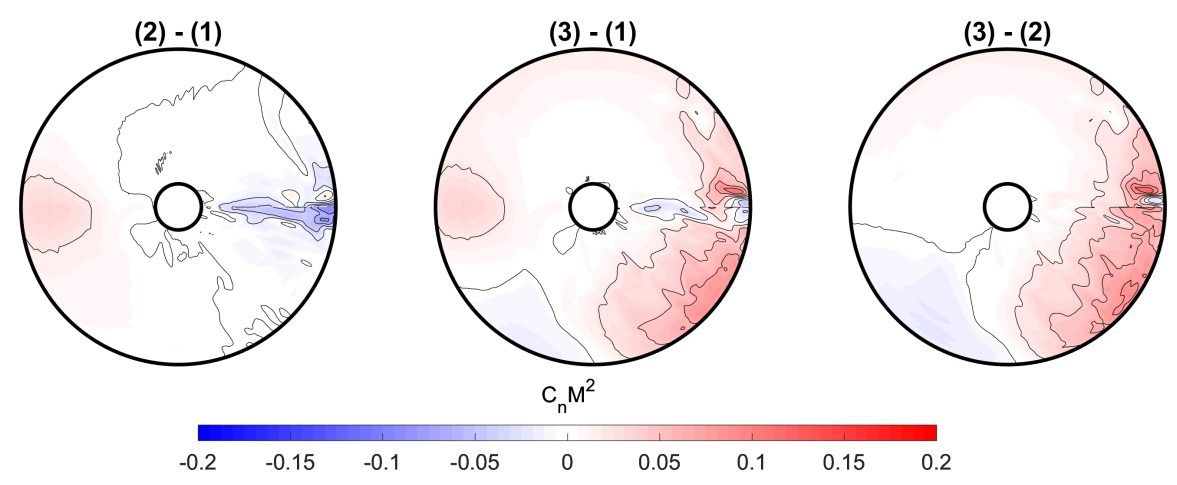

(c)

Figure 7.9: Non-dimensional normal force distribution for lower rotor, rotating clockwise. 
with the full vehicle CFD/CSD coupled approach and the coaxial CFD/CSD coupled approach. The final full vehicle CFD/CSD approach predicts higher lower rotor normal force in the first quadrant over majority of the blade, as well as an increase in normal force on the outboard stations of the blade on the retreating side, quadrants 3 and 4 . The difference between the normal force predicted with the full vehicle and the coaxial CFD/CSD approaches is due to the difference in the predicted blade pitch Fig.7.4(b) and the need to counteract the airframe moments. Figure 7.10 shows the azimuthal distribution of the lower rotor sectional normal force at two radial locations, $49 \% R$ and $98 \% R$, to supplement the contour plots and provide the reader with a more quantitative view of the normal force.
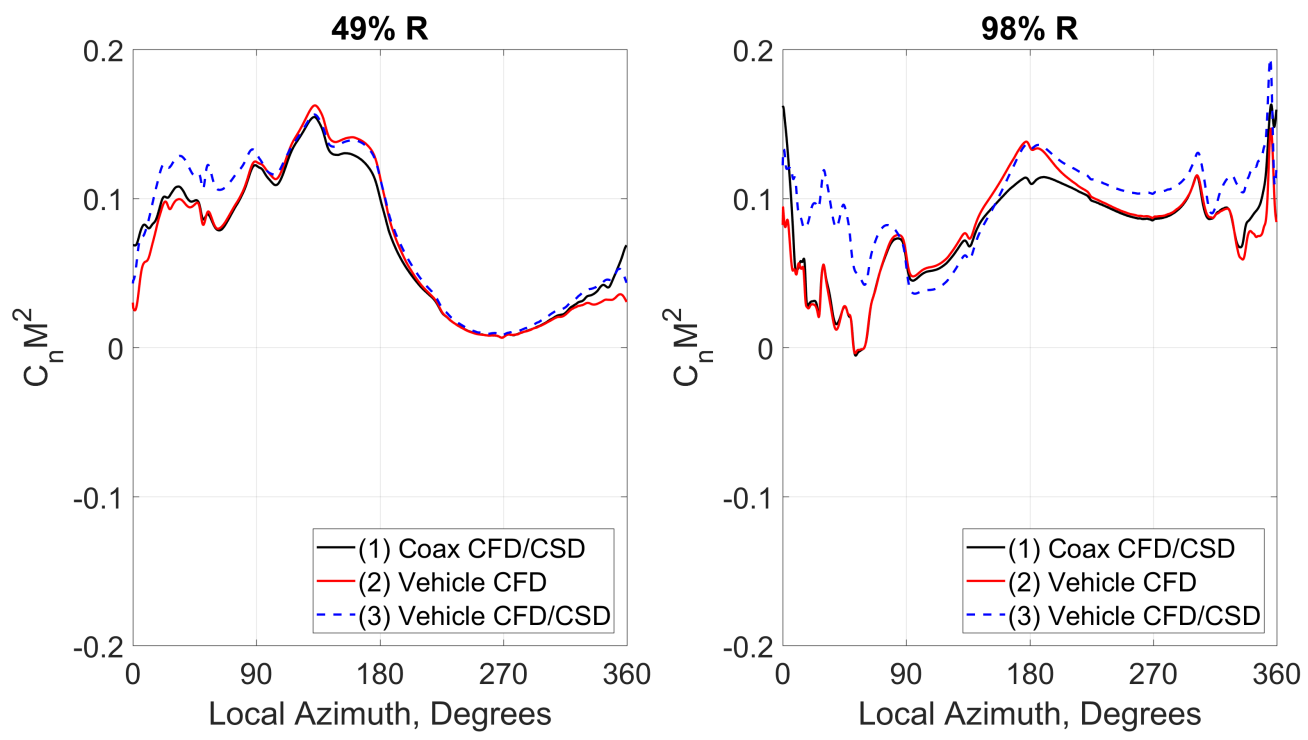

Figure 7.10: Comparison of lower rotor sectional normal force at 150 knots. 


\subsubsection{Chordwise Force - Upper and Lower Rotor}

This subsection examines non-dimensional chordwise force on the upper rotor and lower rotor, obtained using coaxial and full vehicle CFD/CSD coupling methodologies. The non-dimensional chordwise force discussed in this section is in the deformed airfoil frame, positive from the trailing edge to the leading edge.

Figure 7.11 (a) shows the azimuthal sign convention for the upper rotor and the nomenclature of the quadrants. Figure 7.11(b) shows the azimuthal distribution of chordwise force for three datasets: (1) coaxial CFD/CSD, (2) vehicle CFD simulation utilizing main rotor elastic deflections from coaxial CFD/CSD simulation and (3) full vehicle CFD/CSD simulation. It is difficult to recognize the dissimilarities between these datasets, therefore a difference between the datasets is shown in Fig.7.11(c). The first diagram in Fig 7.11(c) shows the difference between dataset (2) and dataset (1) in Fig 7.11(b). The presence of the airframe in the CFD increases the chordwise force on the fore region of the rotor disk, above the airframe, and decreases the chordwise force on the aft portion of the rotor disk. Re-trimming the coaxial rotor with full vehicle CFD/CSD coupling methodology, decreases the blade pitch over the front portion of the rotor disk which in turn decreases the chordwise force. A quantitative view of the changes in chordwise force, over the upper rotor disk, are shown for two radial stations in Fig.7.12.

The sign convention for the lower rotor chordwise force is the same as for the upper rotor, with positive chordwise force from trailing edge towards the leading edge. Again, take note of the direction of the rotation of the lower rotor and the 


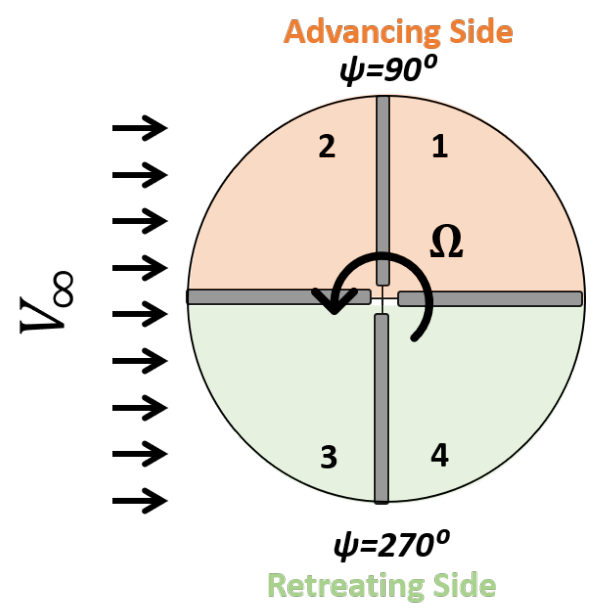

(a)

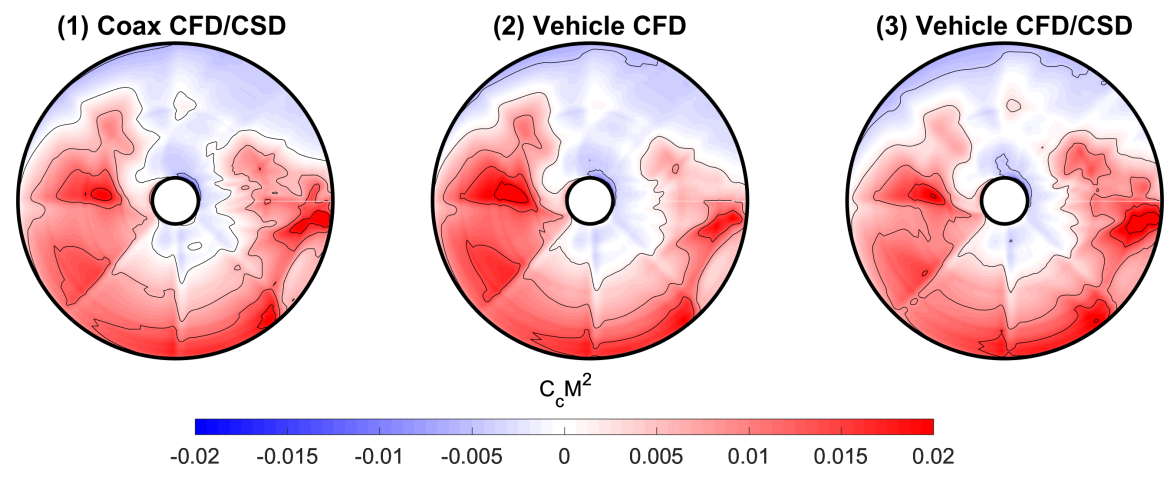

(b)

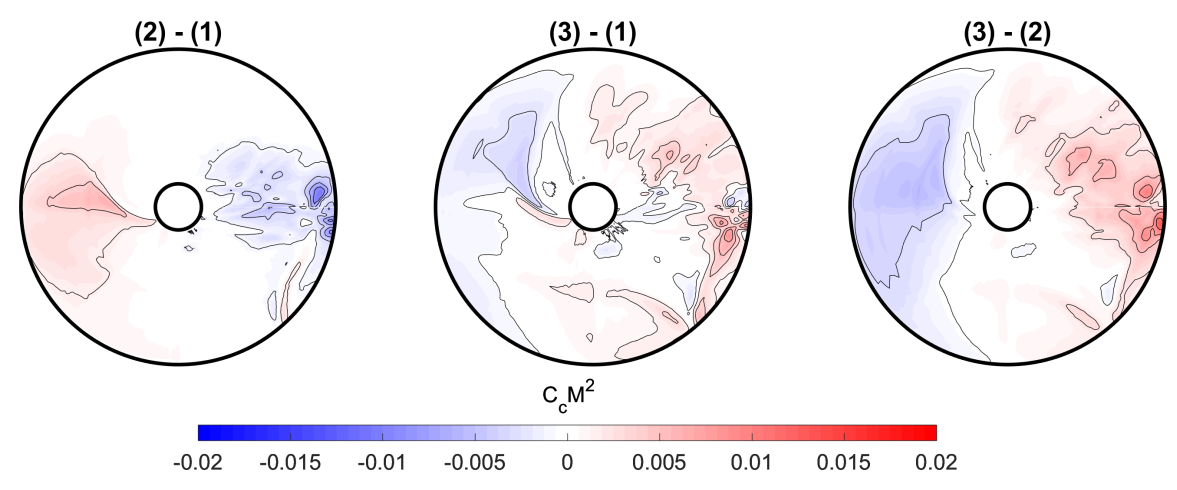

(c)

Figure 7.11: Non-dimensional chordwise force distribution for upper rotor, rotating counter-clockwise. 

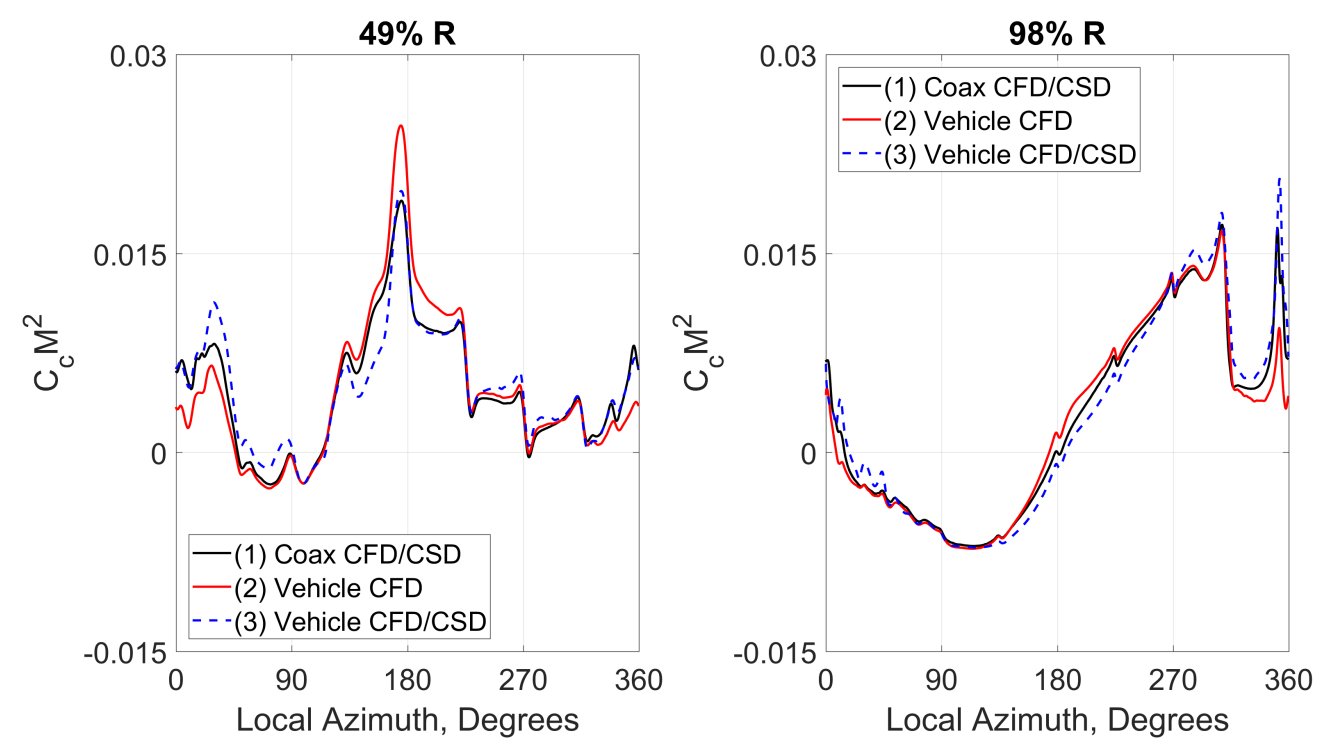

Figure 7.12: Comparison of upper rotor sectional chordwise force at 150 knots.

locations of the advancing and retreating side with respect to the rotor disk, shown in Fig.7.13(a). Figure7.13(b) shows the contours of the azimuthal distribution of the lower rotor chordwise force, in the deformed airfoil frame, for various datasets described above. It is difficult to analyze the differences between the datasets from comparisons in Fig 7.13(b), therefore the differences between the datasets is shown in Fig 7.13 (c). The first diagram in Fig.7.13(c) shows the difference between chordwise force predicted (2) and (1), shown in Fig 7.13(b). The full vehicle CFD/CSD coupling procedure predicts substantially higher chordwise force in the first, third and fourth quadrants on the outboard stations, shown in Fig.7.13(b) and Fig 7.14.

\subsubsection{Pitching Moment - Upper and Lower Rotor}

The objective of this subsection is to identify and analyze the differences in pitching moment that arise from full CFD/CSD coupling approach. The pitching 


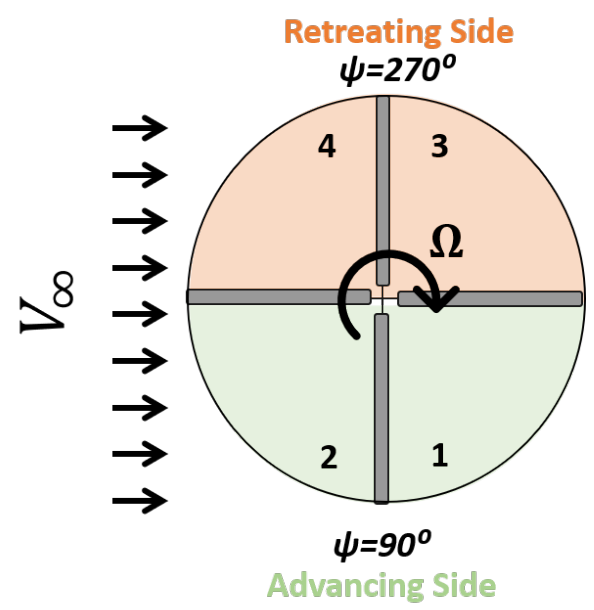

(a)

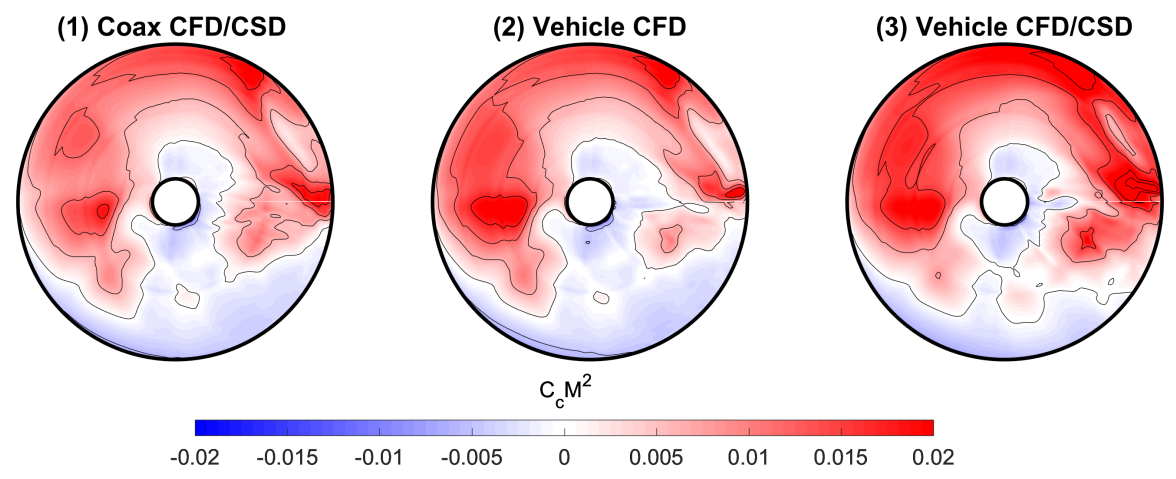

(b)

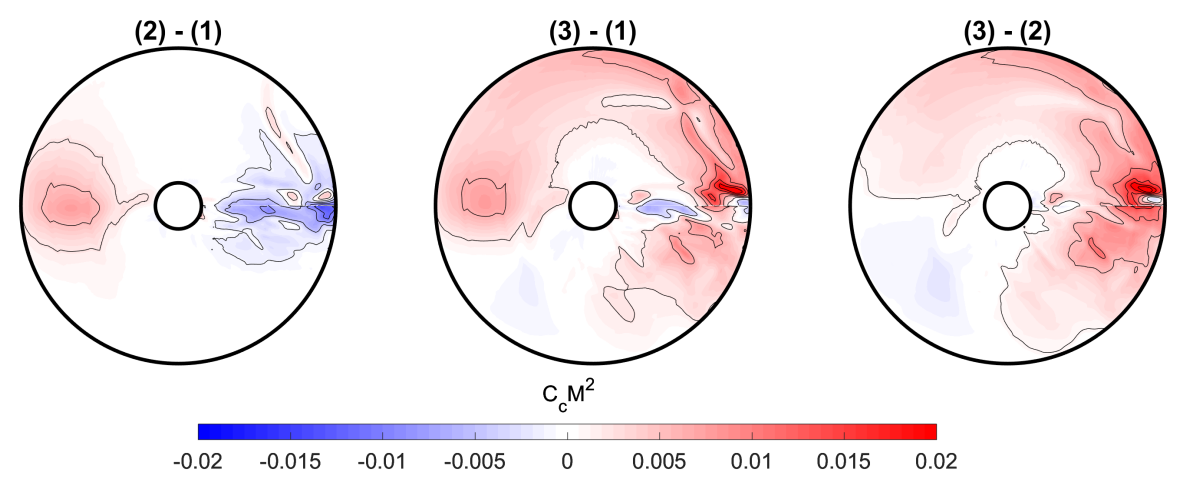

(c)

Figure 7.13: Non-dimensional chordwise force distribution for lower rotor, rotating clockwise. 

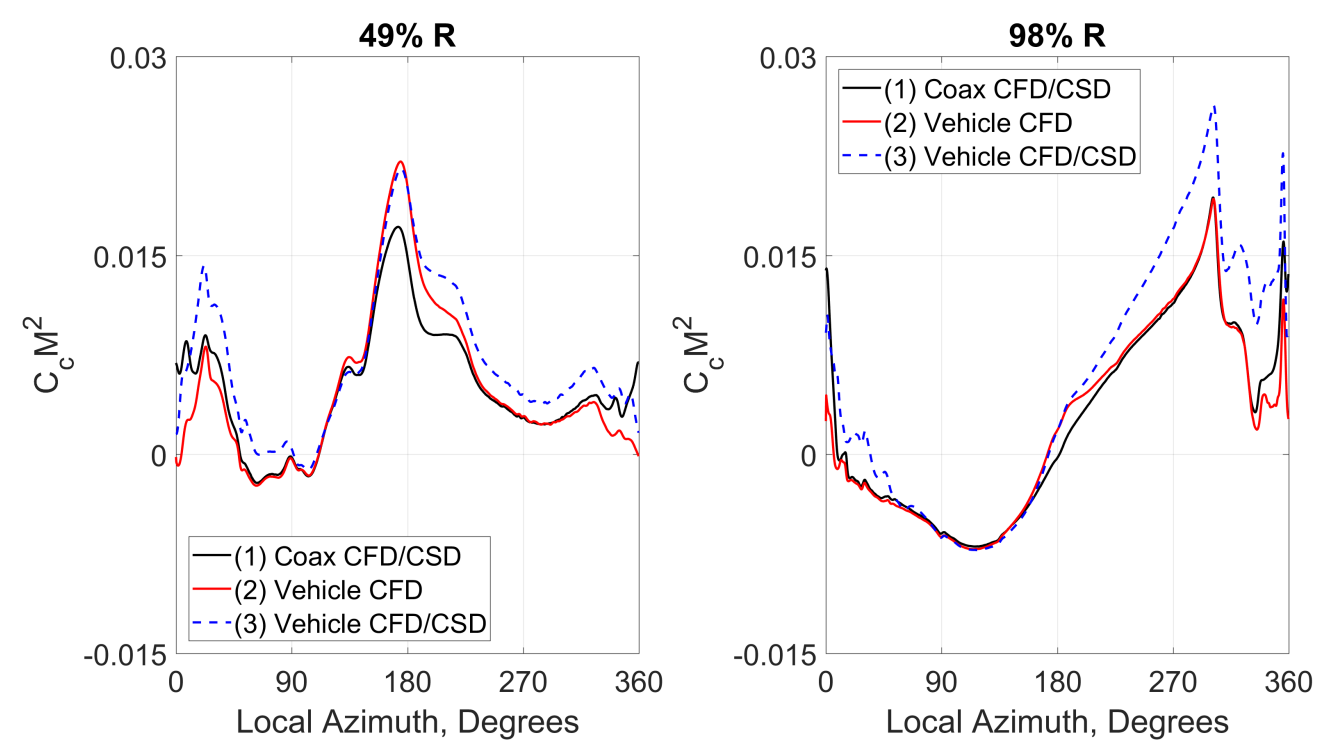

Figure 7.14: Comparison of lower rotor sectional chordwise force at 150 knots.

moment that will be shown in this section is a non-dimensional pitching moment in the deformed airfoil frame. The pitching moment is positive for pitch up motion around the quarter chord line. The pitching moment will be compared for three datasets: (1) coaxial CFD/CSD coupling simulation, (2) vehicle CFD simulation utilizing deflections from coaxial final CFD/CSD coupling simulation and (3) the full vehicle CFD/CSD simulation.

Figure $7.15(\mathrm{~b})$ shows the azimuthal distribution of the upper rotor pitching moment for the three datasets, (1) through (3). Figure 7.15(c) shows the difference between the pitching moment predicted in these datasets. The shedding from the airframe mast in the full vehicle CFD simulation induces unsteady fluctuations in the pitching moment on the aft of the rotor disk, shown in first diagram of Fig.7.15. The full vehicle CFD/CSD simulations predict a decrease in the nose-down pitching moment on the advancing side, shown in the second diagram of Fig.7.15(c) as well 


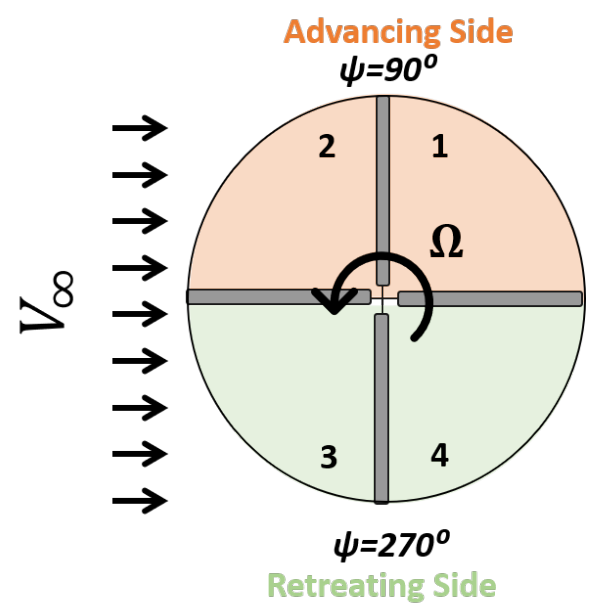

(a)

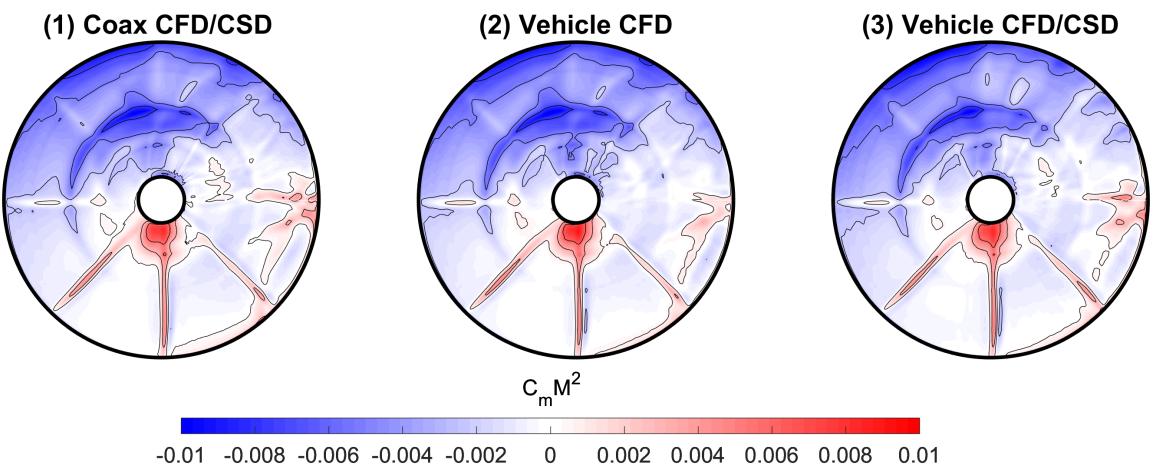

(b)

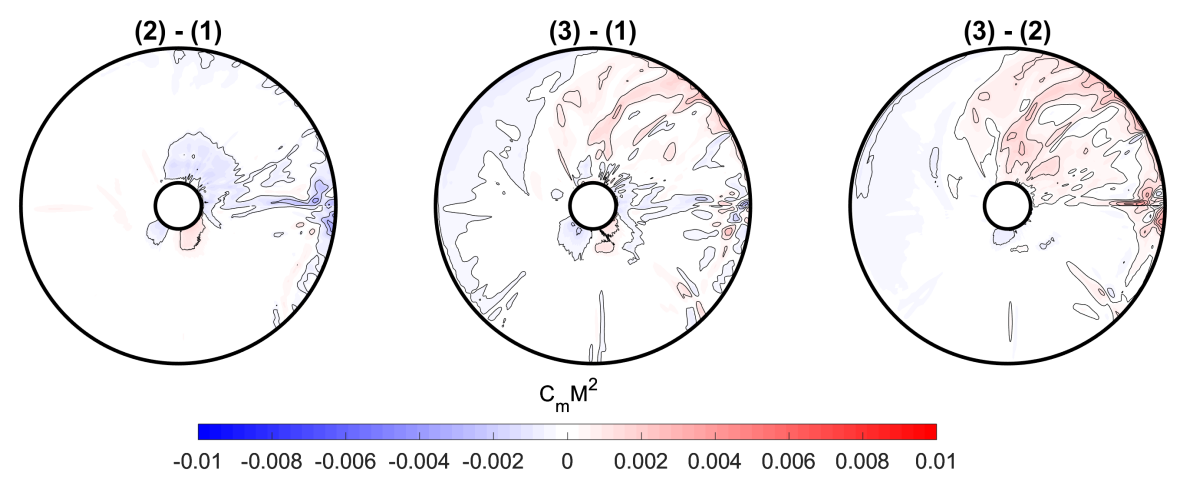

(c)

Figure 7.15: Non-dimensional pitching moment distribution for upper rotor, rotating counter-clockwise. 

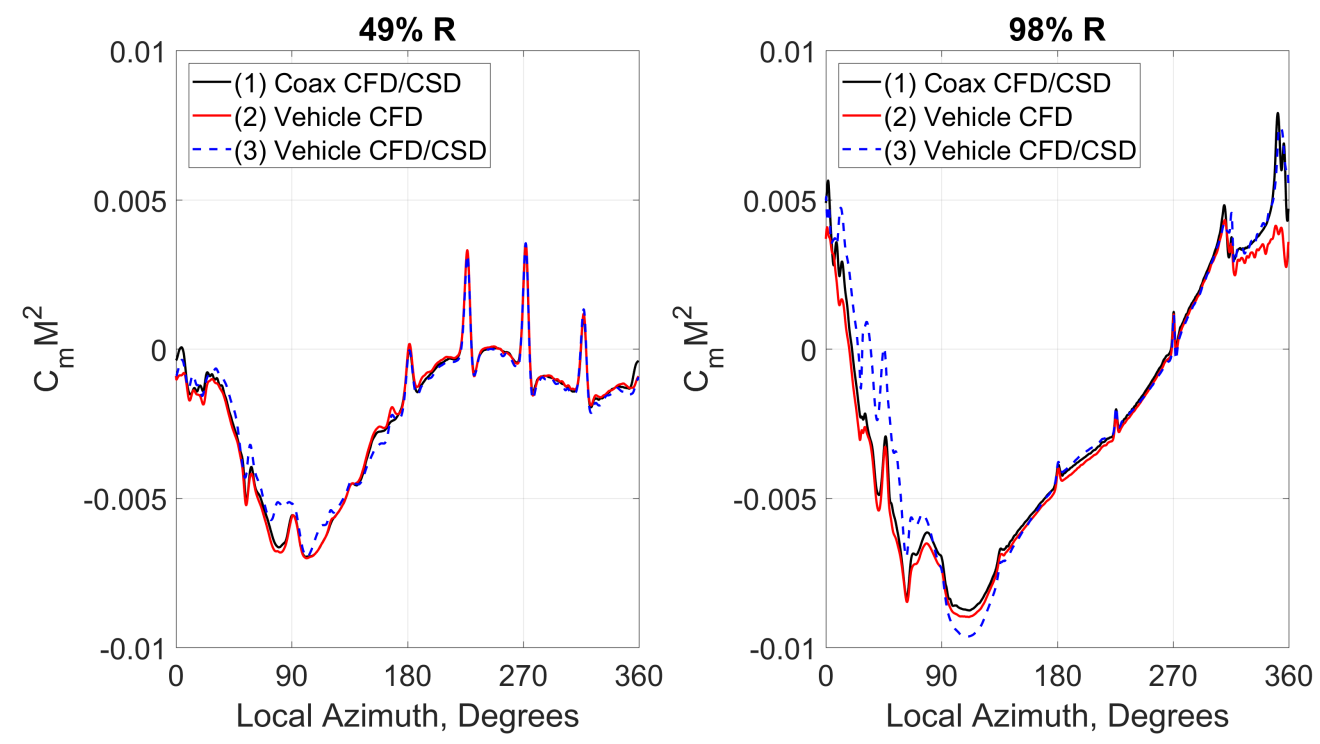

Figure 7.16: Comparison of upper rotor sectional chordwise force at 150 knots.

as in Fig 7.16.

Figure 7.17(b) shows the lower rotor pitching moment for the three datasets, while Fig. 7.17(c) shows the difference in the lower pitching moment for the three datasets. The difference between the lower rotor pitching moment predicted using the full vehicle CFD/CSD coupling and the coaxial CFD/CSD coupling is shown in the second diagram in 7.17(c). Majority of the differences occur on the aft of the rotor disk, in the first and the second quadrants. In the first quadrant, full vehicle CFD/CSD predicts lower nose down pitching moment compared to the coaxial CFD/CSD prediction. In the second quadrant there is a nose down signature which may be due to a vortex being pinched off of the retreating blade. 


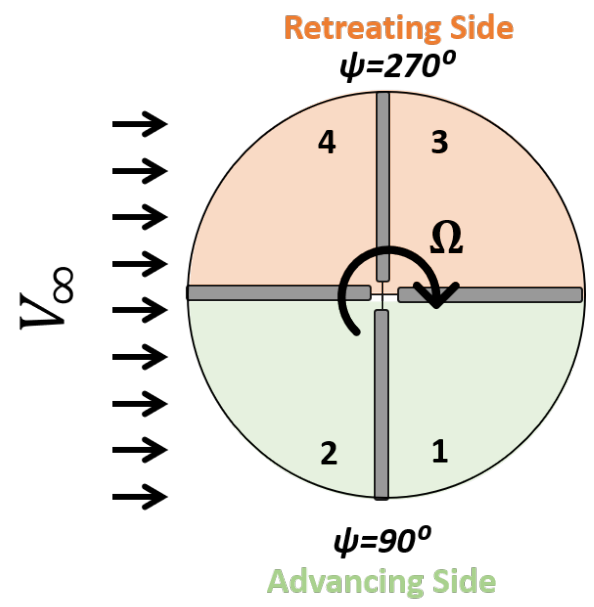

(a)

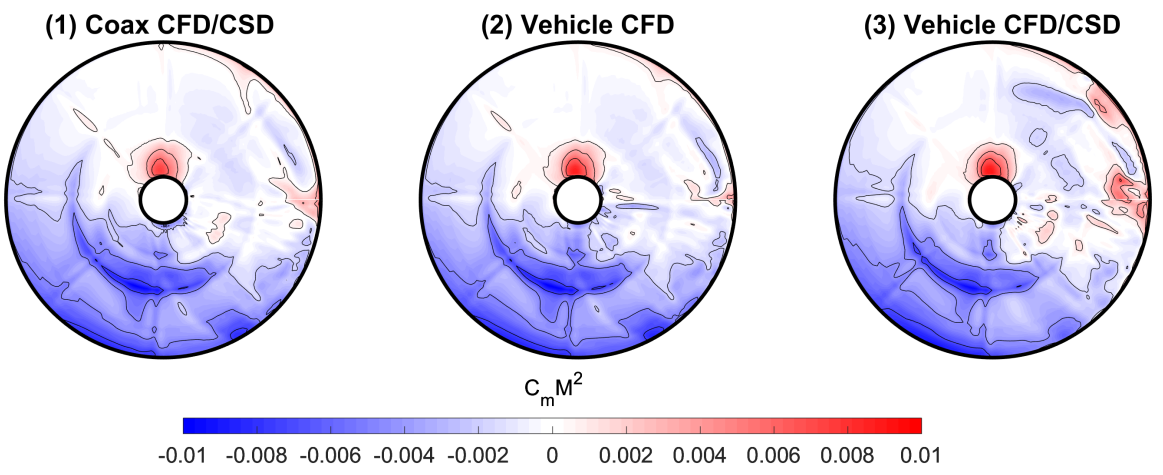

(b)

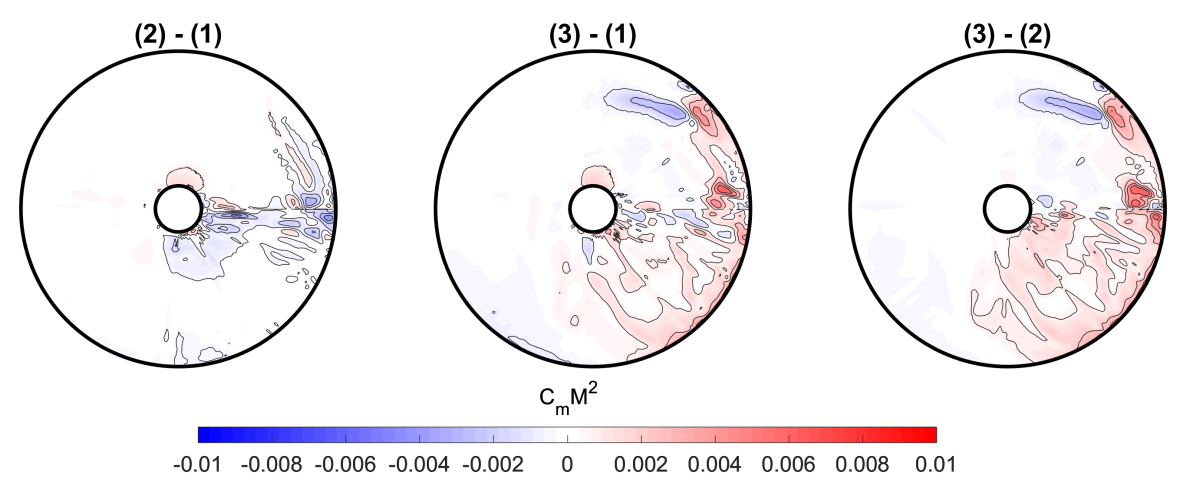

(c)

Figure 7.17: Non-dimensional pitching moment distribution for lower rotor, rotating clockwise. 

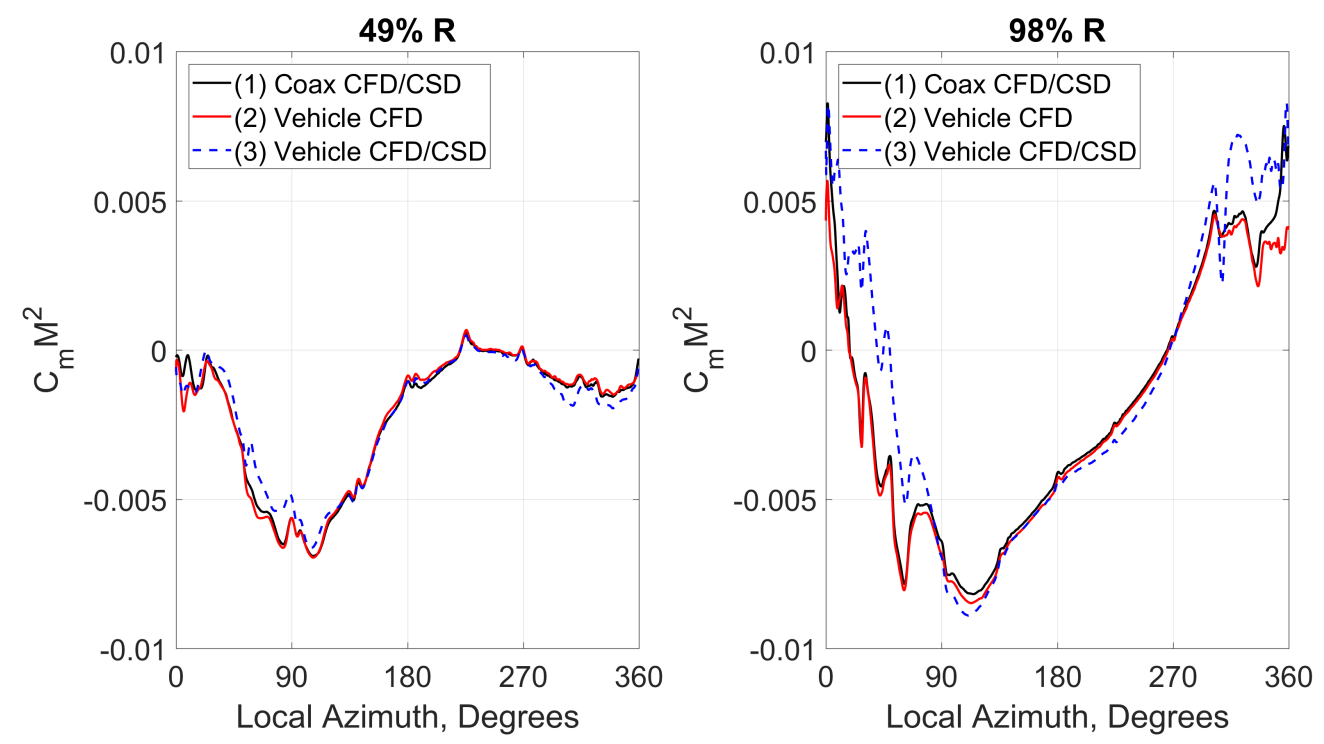

Figure 7.18: Comparison of lower rotor sectional pitching moment force at 150 knots.

\subsection{Structural Blade Loads}

This section compares the main rotor structural blade loads obtained with the coaxial CFD/CSD coupling approach and the full vehicle CFD/CSD coupling approach. The blade loads consist of the flap bending moment, chordwise bending moment and torsional bending moment. The blade loads are obtained using the force summation method, by integrating the aerodynamic, centrifugal and inertial forces from the tip of the blade to the radial station of interest.

Figure 7.19 compares the flap bending moment obtained from the two CFD/CSD coupling approaches. This figure shows the flap bending moment near the root of the rotor blade, $r / R=18.6 \% R$, as the blade travels around azimuth. The flap bending moment is closely related to the normal force shown in Figs.7.7 and 7.9, as well as the vertical components of the inertial and centrifugal forces acting on the blade. 


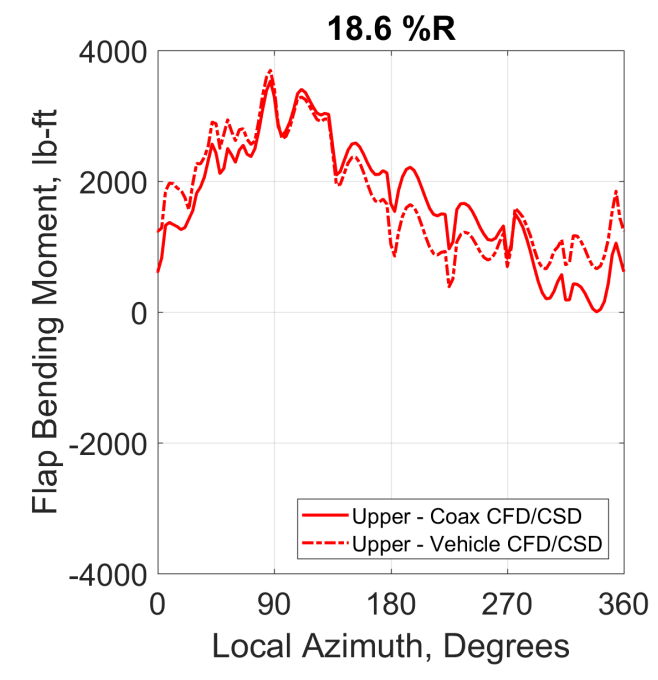

(a)

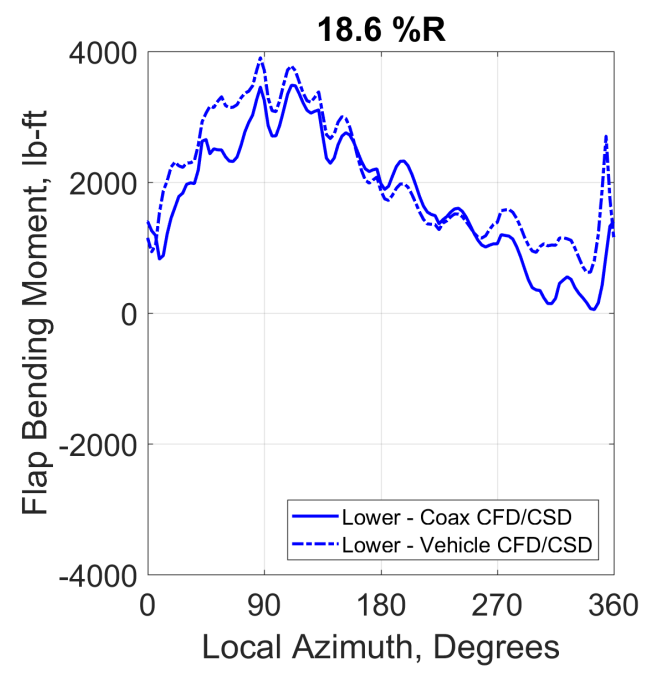

(b)

Figure 7.19: Upper and lower rotor flap bending moment obtained using coaxial and full vehicle CFD/CSD coupling at 150 knots.

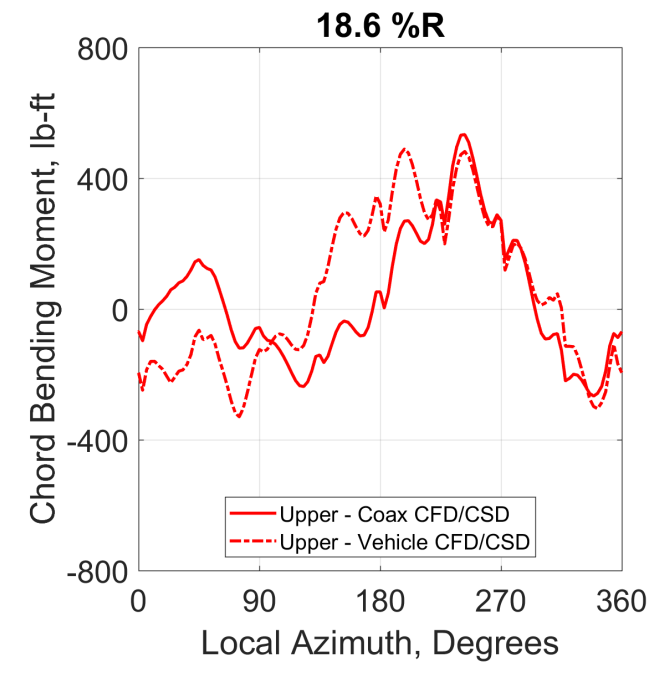

(a)

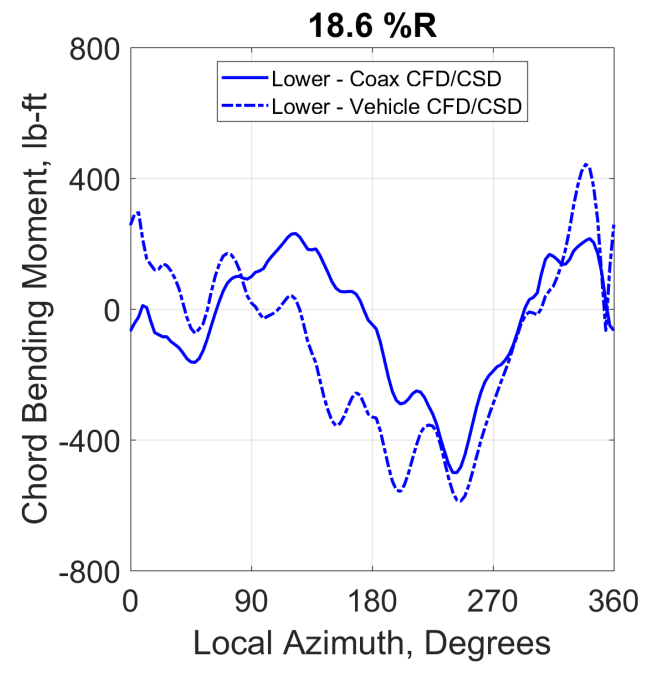

(b)

Figure 7.20: Upper and lower rotor chord bending moment obtained using coaxial and full vehicle CFD/CSD coupling at 150 knots. 


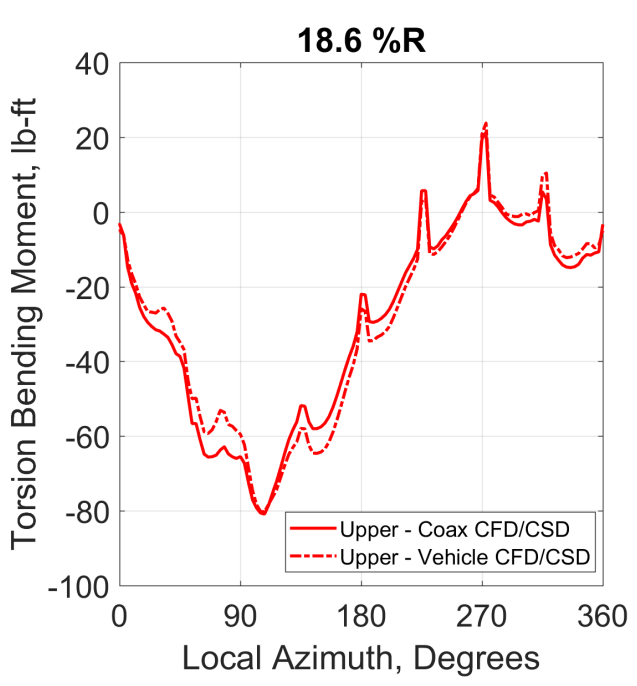

(a)

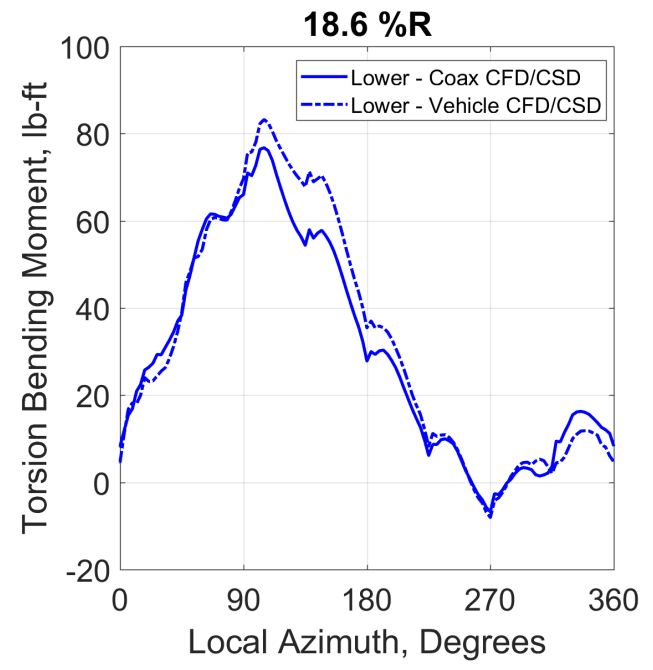

(b)

Figure 7.21: Time history of the upper and lower rotor torsion bending moment obtained using coaxial and full vehicle CFD/CSD coupling at 150 knots.

A positive flap bending moment is defined with the upper surface in compression (blade flapping up). Both CFD/CSD coupling approaches predict similar trends. The maximum flap bending moment occurs on the advancing side of the rotor disk due to high normal force distribution. The flap bending moment decreases as the blade travels around the azimuth. The decrease is reflective of the normal force decrease in the reverse flow region. However, the flap bending moment does not become zero because there is a sufficient normal force on the outboard stations of the blade on the retreating side. The flap bending moment does approach zero in the third quadrant because there is a decrease in the normal force on the outboard stations of the blades indicative of the dynamic stall vortex pinching off of the blade. Both CFD/CSD approaches predicted very similar maximum flap bending moment magnitudes. The full vehicle CFD/CSD approach predicts slightly higher flap bend- 
ing moment on the aft portion of the rotor disk, as the blade travels through the first and the third quadrants. The blade pitch in the first and third quadrant is higher for the rotor trimmed with full vehicle CFD/CSD coupling procedure, shown in Fig 7.4(a) and(b), producing more normal force in those quadrants. The full vehicle CFD/CSD predicts lower flap bending moment on the fore portion the rotor disk, as the blade travels through the second and the third quadrant. The blade pitch in the second and the fourth quadrant is lower for the rotor trimmed with full vehicle CFD/CSD coupling procedure, producing less normal force in these locations, therefore lower flap bending moment. The oscillatory nature of the flapping moment is due to the high frequency content in the normal force.

Figure 7.20 shows the chordwise bending moment comparisons, at $r / R=$ $18.6 \% R$, for the coaxial and the full vehicle CFD/CSD coupling methodologies. The chordwise bending moment is positive for blade lead. Both methods predict the maximum chordwise bending moment to be located on the retreating side of the rotor disk, a result of increased profile drag in the reverse flow region. Pronounced differences in the sign of the chordwise bending moment are observed on the advancing side of the rotor disk, with full vehicle CFD/CSD coupling predicting upper rotor have negative chordwise bending moment in the first quadrant and positive chordwise bending moment in the second quadrant. The coaxial CFD/CSD coupling predicted the opposite to be true.

Figure 7.21 compares the torsion bending moment for coaxial and full vehicle CFD/CSD coupling methodologies. The torsion bending moment is shown at $18.6 \%$ radial location. A positive torsion bending moment is defined positive nose-up for 
upper rotor and nose down for lower rotor. An 8P signature can be observed in the time-history, with the high impulsive peaks being more prominent on the retreating side. The $8 \mathrm{P}$ signature is an interactional aerodynamic phenomena, occurring at blade crossings, which would not be evident in the structural blade loads without the CFD/CSD coupling.

\subsection{Pusher Propeller}

The auxiliary propeller is treated as a rigid rotor with only a collective inputs. Every coupling cycle the auxiliary propeller was trimmed in isolation using a climb regime in the wind tunnel mode. The auxiliary propeller power, zero roll moment and zero pitching moment are the trim targets. After the initial trim, a delta coupling method was applied. The difference between the CFD auxiliary propeller power and the target power is calculated and added on to the difference from the previous iterations. The cumulative delta is then applied into the trim process. The output of the auxiliary propeller trim is the collective and thrust. The propeller thrust is an input into the full CFD/CSD coupling trim procedure, modifying the speed schedule which specifies the thrust power at a specific speed. In the previous section the auxiliary propeller thrust was calculated using momentum theory. The propeller collective is an input into the CFD simulation.

Figure 7.22 (a) compares the flight test auxiliary propeller power, published in Ref. [6], and the predicted auxiliary propeller power obtained from full vehicle CFD/CSD approach. Initially the CFD power is over-predicted. As the coupling 

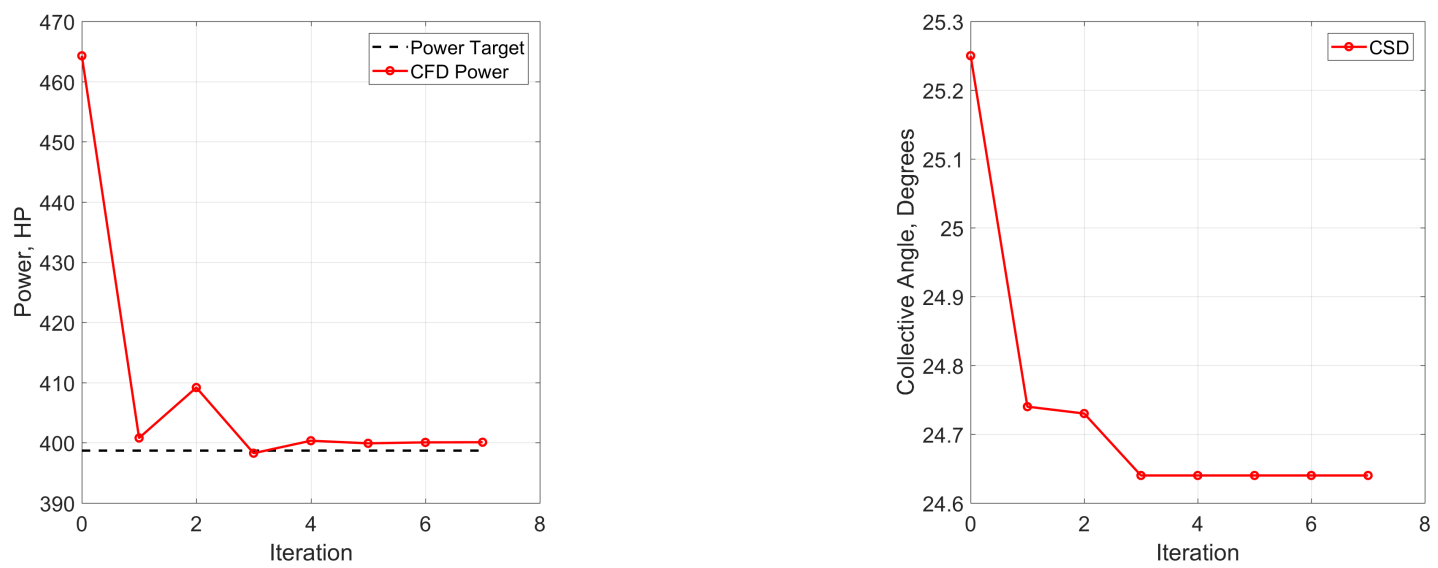

Figure 7.22: Convergence history for propeller power and collective.

cycles advance the predicted propeller power approaches the target propeller power. At the third coupling cycle there was a sufficiently small difference between the predicted and the flight test propeller power, therefore no further coupling iterations were performed for the auxiliary propeller and the propeller was no longer re-trimmed. The collective was kept constant, same collective as the third iteration, for full configuration CFD/CSD coupling cycles. Even though the collective remained constant, the propeller power went up due to the change in the fuselage pitch attitude during the full CFD/CFD coupling. However, since this change in power was relatively small and the CFD power closely matched the target power, it was deemed acceptable not to re-trim the propeller any further. 


\section{Results Part III: Fundamental Understanding of Interac- tional Aerodynamics of Compound Coaxial Helicopter}

This chapter examines the aerodynamic interactions between various components of the high-speed compound helicopter in steady level flight at 150 knots, using Helios/OVERFLOW simulations. The objective of the chapter is to identify the aerodynamic interactions between various components and determine their effect on the performance and the vibratory aerodynamic airloads. The components that are investigated are coaxial rotor system, airframe and the auxiliary propeller. CFD simulations were performed with individual components and component combination, shown in Fig 8.1 . The upper and lower rotor deflections from full vehicle CFD/CSD coupling, discussed in previous chapter, were used for all simulations that included the coaxial rotor system. The airframe includes the pylon, mast and empennage. The pitch attitude of the helicopter was also obtained in the previous section using full vehicle CFD/CSD coupling methodology and it did not change for the interactional aerodynamic studies. The auxiliary propeller blade pitch was set based on the final trim from full vehicle CFD/CSD simulations. 


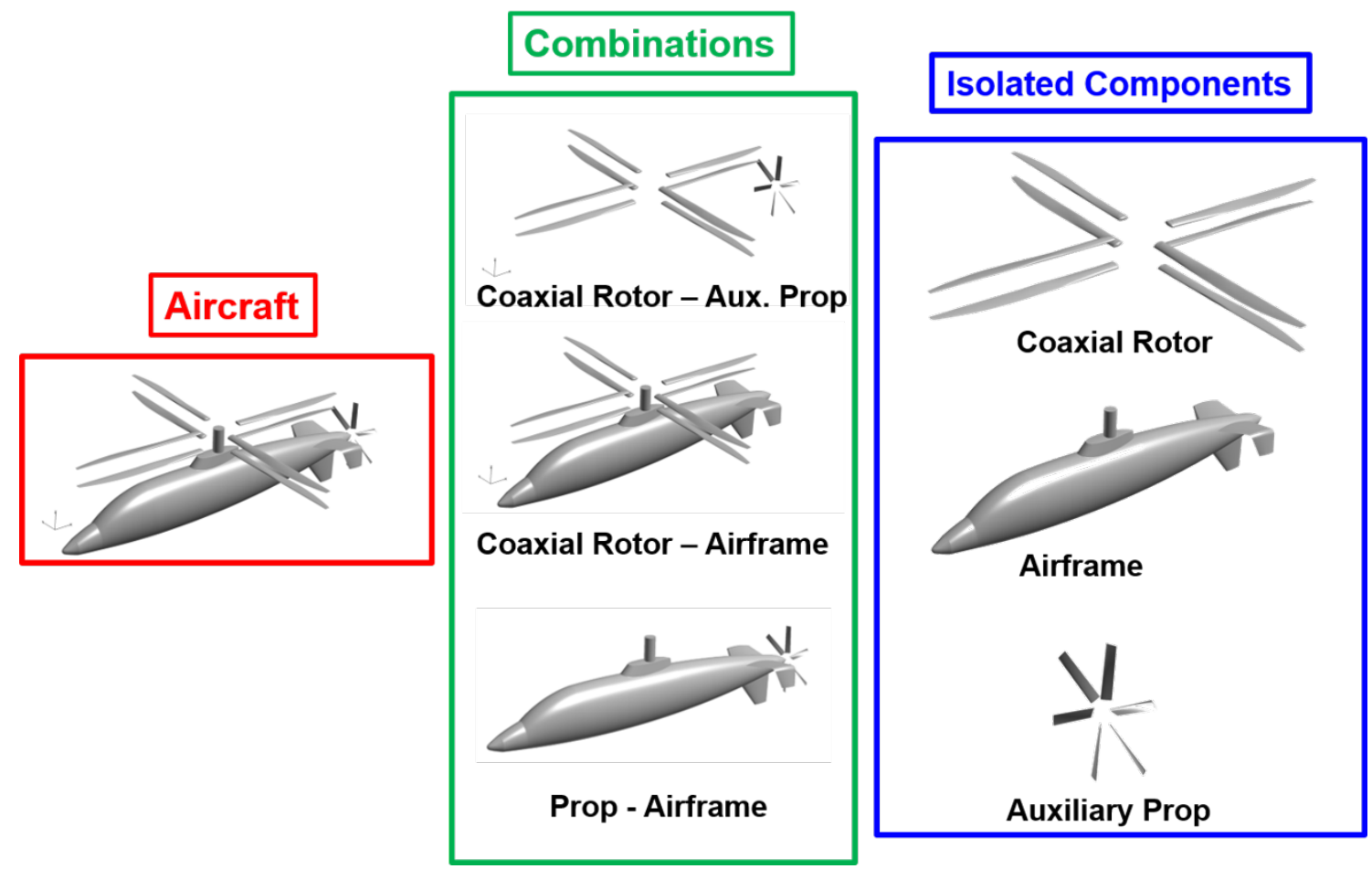

Figure 8.1: Helicopter components and isolated parts interactional aerodynamic study 
Table 8.1: CFD interactional aerodynamics case

\begin{tabular}{ccccccc}
\hline \hline$V_{\infty}($ knots $)$ & $\mu$ & MR RPM & $M_{\text {adv.tip }}$ & $\operatorname{LOS}(\%)$ & Blade Modes & Harmonics \\
\hline 150 & 0.41 & 446 & 0.79 & 11.3 & 6 & 8 \\
\hline \hline
\end{tabular}

\subsection{Interactional Aerodynamics Affecting the Upper Rotor}

This section examines the aerodynamic interactions affecting the performance of the upper rotor. CFD simulations of the upper rotor in isolations as well as in conjunction with other helicopter components, shown in Fig.8.2(a), were performed at 150 knots.

Figure 8.2 (b) shows the normal force distribution for the geometries shown in Fig 8.2 (a). Isolated upper rotor results in fairly steady normal force around the rotor azimuth, with significant amount of normal force produced over the aft of the rotor disk. Including the lower rotor in the CFD simulations results in unsteadiness in the normal force and drop off in normal force every 45 degrees, where blades cross each other. Including the airframe in CFD simulations results in an upwash over the aft portion of the airframe, decreasing the normal force over the aft of the rotor disk. No significant change is observed in normal force with the addition of the auxiliary propeller.

Figure 8.2 (c) shows the chordwise force, in the airfoil frame, for the upper rotor. The chordwise force is positive from the trailing to leading edge. CFD simulation of isolated upper rotor results in high distribution of positive chordwise force in the fourth quadrant, which decreases with the inclusion of the lower rotor 
in the simulations. Periodic unsteadiness, which was observed in the normal force distribution, is also observed in the chordwise force. This is due to the proximity between the upper rotor blades and the wake of the lower rotor, note that at this speed the rotor disk is tilted aft.

Figure $8.2(\mathrm{~d})$ shows the upper rotor non-dimensional quarter-chord pitching moment distribution, in the airfoil frame. Positive pitching moment for upper rotor represents the airfoil section pitching up about the quarter chord of the blade. Including the lower rotor in the CFD simulations introduces unsteadiness into the pitching loads, as well as an 8P pitch-up impulsive spikes on the retreating side of the disk.

The $8 \mathrm{P}$ impulsive changes in airloads originate when the blades of the upper rotor cross with the blades of the lower rotor. During the crossing of the blades, the upper rotor blades transverse through a low pressure region above the lower blades, which reduces the pressure under the upper rotor blades resulting in a momentary loss of lift.

Figure 8.4 shows the upper rotor aerodynamic hubloads in frequency domain, for various configurations shown in Fig 8.2 (a). The positive axial hub force is towards the tail of the aircraft, the positive side hub force is starboard and the positive vertical hub force is up. The positive hub roll moment is starboard side up, positive pitching hub moment is nose up and positive yaw hub moment is clockwise.

For a coaxial configuration there is a significant increase in an $8 \mathrm{P}$ yaw moment, shown in Fig.8.4(f), which is caused by the $8 \mathrm{P}$ periodic variation in both the lift force and the drag force. The $8 \mathrm{P}$ yaw moment is a almost half of the steady yaw 
moment. The addition of the airframe or the propeller did not have significant effect on the $8 \mathrm{P}$ yaw moment. The $8 \mathrm{P}$ contribution to the roll moment, shown in Fig. $8.4(\mathrm{~d})$, is also significant.

The steady side hub force, shown in Fig 8.4(b) and pitching hub moment, shown in Fig.8.4(e), are strongly effected by the consideration of the lower rotor and airframe. The steady negative side force (towards port side) increases with addition of lower rotor and airframe, this is due to the proximity of lower rotor wake and the mast wake to the upper rotor. The steady pitching hub moment changes sign and significantly decreases in magnitude with addition of the airframe in the CFD. Take note that the rotor blade deflections which are used for all the upper rotor cases, Fig.8.2(a), were obtained using full vehicle CFD/CSD coupling discussed in previous section. Therefore, these deflections do not represent trimmed state for the isolated rotor and coaxial rotor system. Using the deflections from full vehicle CFD/CSD coupling for an isolated rotor and coaxial rotor cases results in a nose down pitching moment. However, when the airframe is included in the CFD simulations, there is an upwash over the nose of the aircraft and a downwash over the tail, this phenomena increases the nose up pitching moment hub and decreases the nose down pitching moment. 


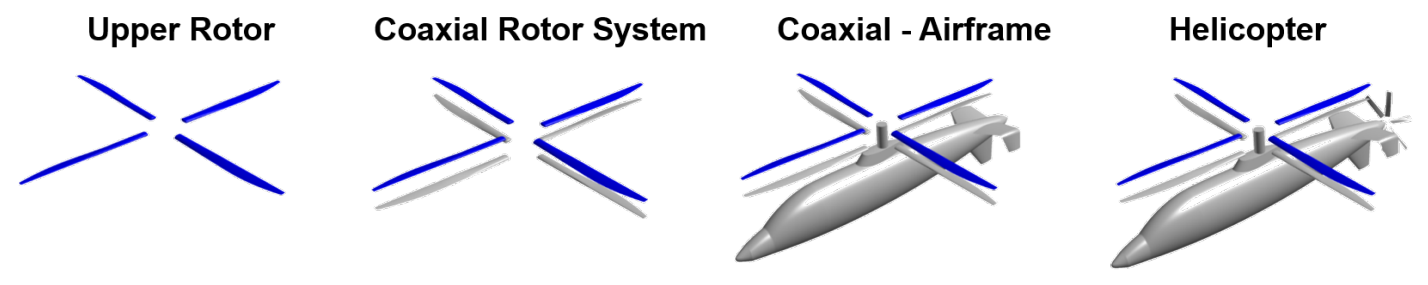

(a) Geometries in CFD

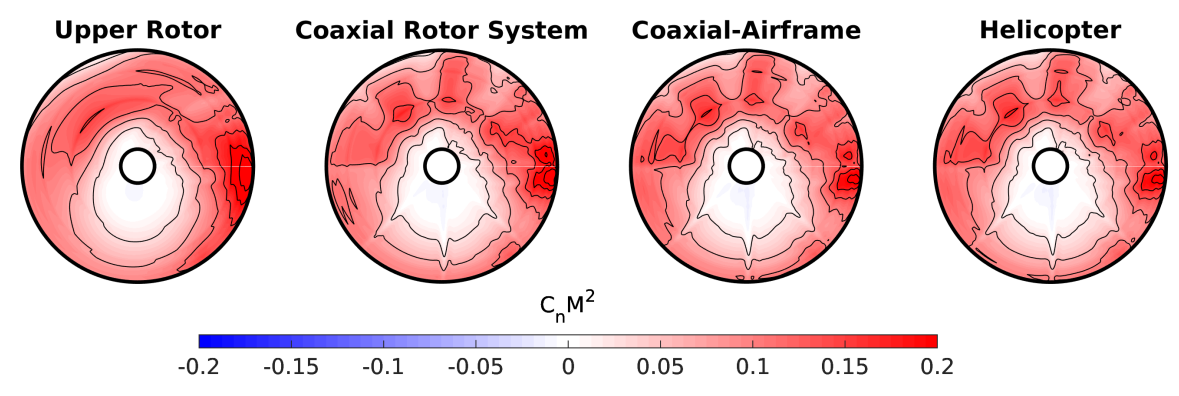

(b) Normal force

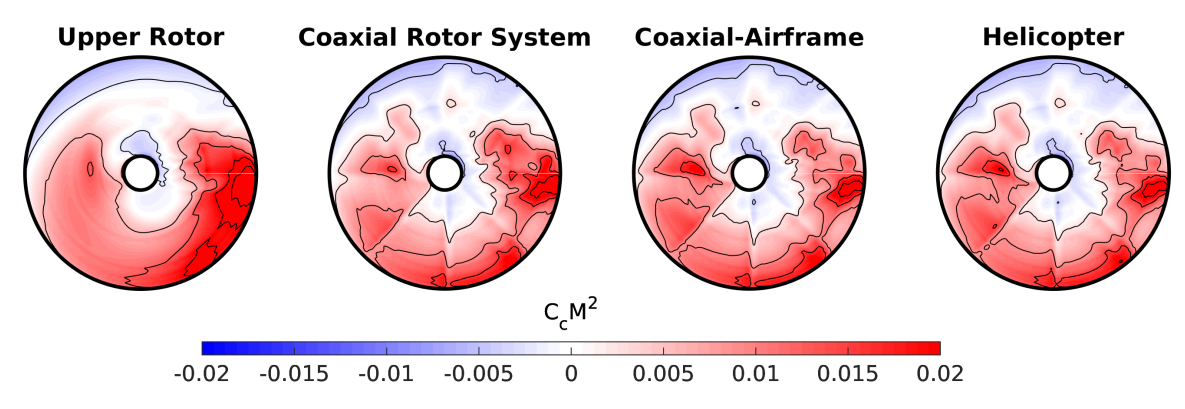

(c) Chordwise force

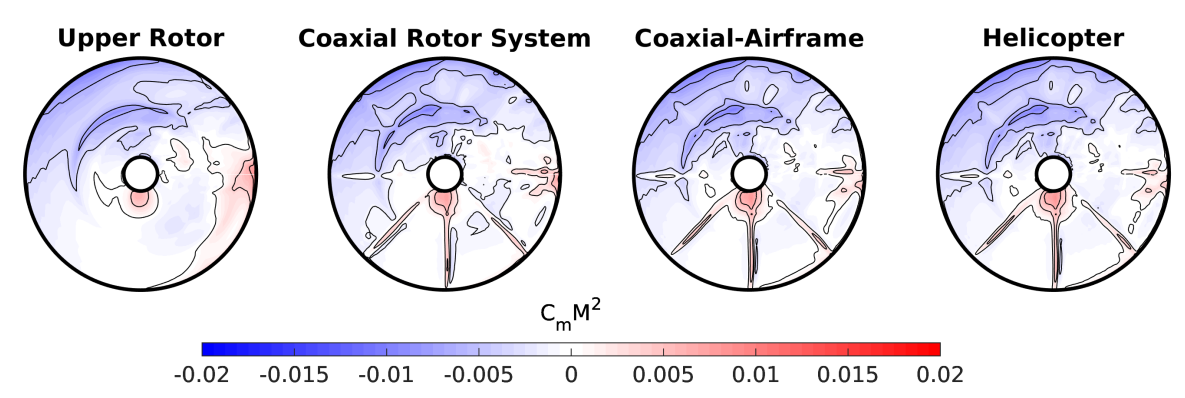

(d) Pitching moment

Figure 8.2: Aerodynamic interactions effects on the upper rotor. 

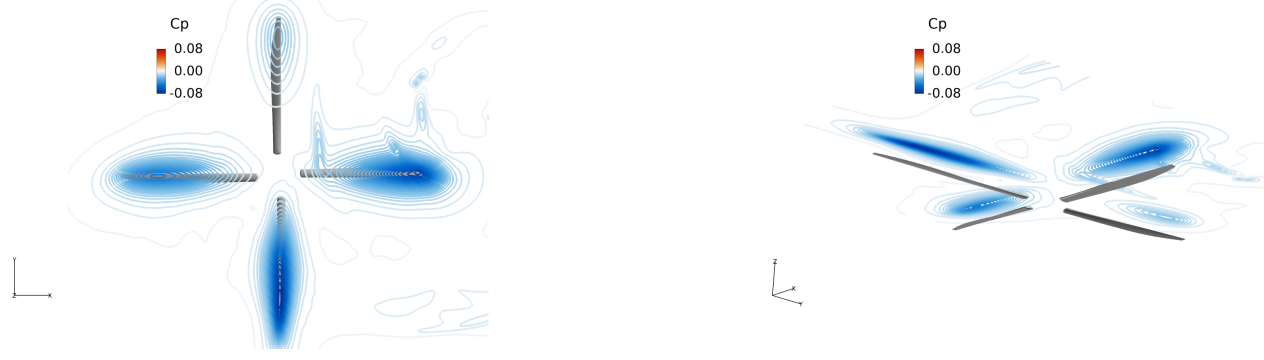

Figure 8.3: Pressure above the lower rotor at the disk plane of the upper rotor at 150 knots.

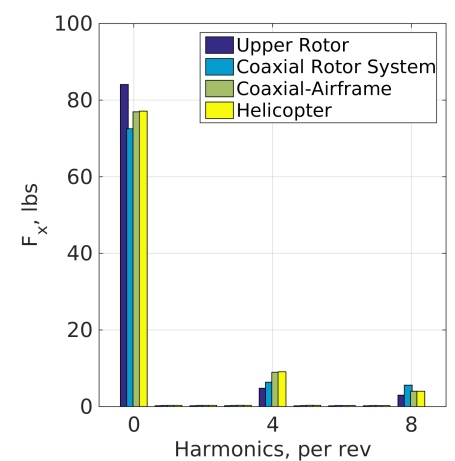

(a)

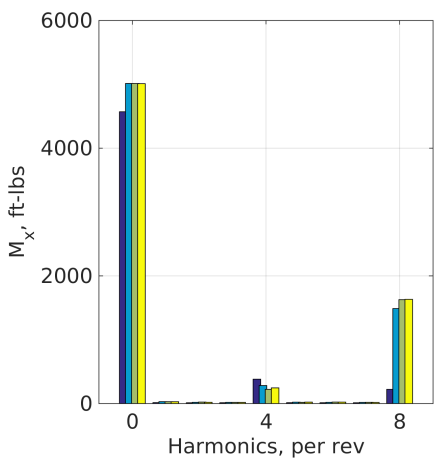

(d)

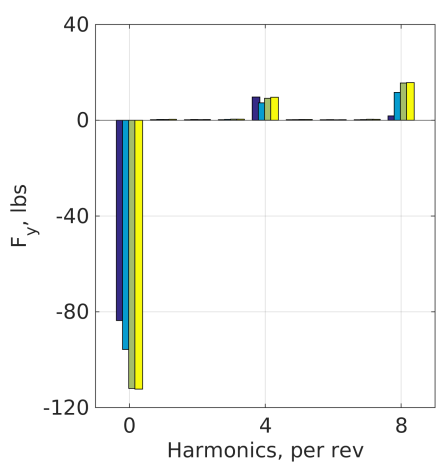

(b)

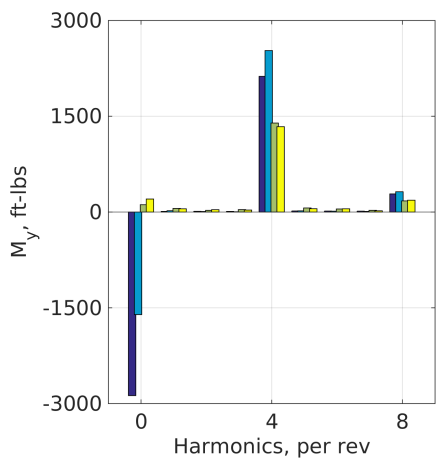

(e)

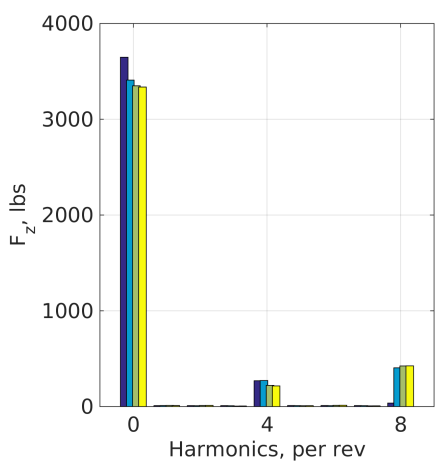

(c)

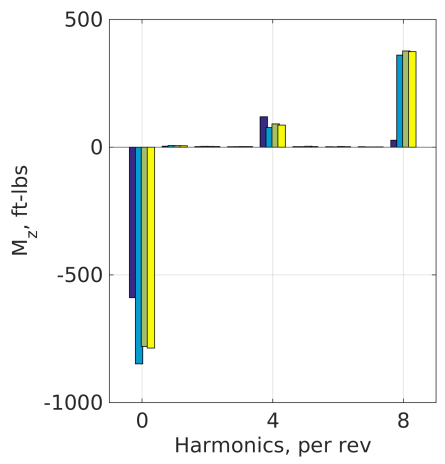

(f)

Figure 8.4: Upper rotor hubloads for various configurations shown in Fig. 8.2(a) at 150 knots. 


\subsection{Interactional Aerodynamics Affecting the Lower Rotor}

This section examines the aerodynamic interactions affecting the performance of the lower rotor. CFD simulations of the lower rotor in isolations as well as in conjunction with other helicopter components, shown in Fig 8.5(a), were performed at 150 knots.

Figure 8.5(b) shows the normal force azimuthal distribution, positive in the upward direction, for the isolated lower rotor as well as in combination with other helicopter components. Take note that the lower rotor rotates in the clockwise direction. The addition of upper rotor into the CFD simulation introduces loss in normal force every 45 degrees on the advancing side, starting at midspan of the blade and extending to the tip. The difference between the normal force on the lower rotor in the coaxial system and the lower rotor in isolation is shown in Fig.8.6(a), labeled CRS-LR. This is due to the blade crossing, during which the lower rotor blades pass under the upper rotor blades, the pressure on the top side of the lower rotor blades increases. Including the airframe in the CFD, increases the normal force on the front of the rotor disk due to the upwash from the airframe. The normal force on the aft of the rotor disk decreases due to the downwash from the airframe. The effect of the mast wake is also observed on the inboard sections of the blade at $\psi=0$ degrees, as loss in lift. The difference in normal force between coaxial rotor system airframe combination and coaxial rotor system is shown in second column of Fig.8.6(a), labeled CA-CRS.

Figure 8.5.(c)shows the azimuthal distribution of the chordwise force on an 
isolated lower rotor and lower rotor in combination with other helicopter components, shown in Fig 8.5(a). For lower rotor the chordwise force, in an airfoil frame, is positive towards the trailing edge. The addition of the airframe to the lower rotor CFD simulations increases the chordwise force on the aft portion of the rotor disk and introduces some unsteadiness, shown in Fig.8.6. The unsteadiness results from the blades interacting with the mast wake.

Figure 8.5(d) shows the azimuthal distribution of the pitching moment, in airfoil frame of reference, for lower rotor. The pitching moment, for lower rotor is positive nose down and negative nose up. Inclusion of the airframe in the CFD doesn't introduce significant differences in terms of pitching moment on the lower rotor, mostly unsteadiness at the rear of the rotor disk.

A significant difference which appears in normal force, chordwise force and pitching moment, Fig.8.5 and Fig, 8.6 is located in the third quadrant of the rotor disk. For an isolated rotor there is a abrupt change in chordwise force and pitching moment on the midspan of the retreating blade. This could be attributed to a dynamic stall vortex pinching off of the retreating blade. This phenomena is reduced when the upper rotor is included in the CFD. The increase in the pressure above the lower rotor (due to the presence of the upper rotor), may attribute the attachment of the vortex.

Figure 8.7 shows the aerodynamic hubloads for the lower rotor in isolation as well as in combination with other helicopter components. Inclusion of the upper rotor results in increased $8 \mathrm{P}$ harmonic for all hub forces and moments. The $8 \mathrm{P}$ arises from the interactions of the lower and the upper rotor blades when they cross 
over. There is an increase in the axial force with addition of the upper rotor and the airframe. The unsteady wake from the mast contributes to the increase in drag on the lower rotor.

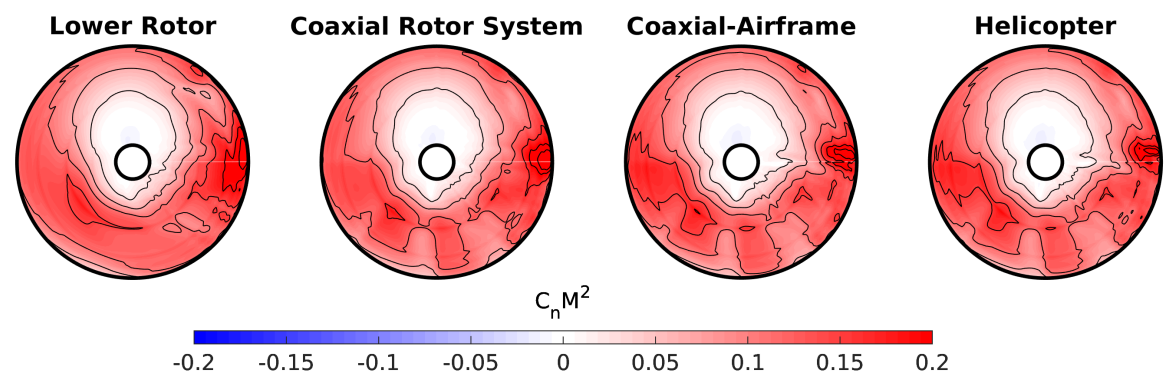

(a) Normal force

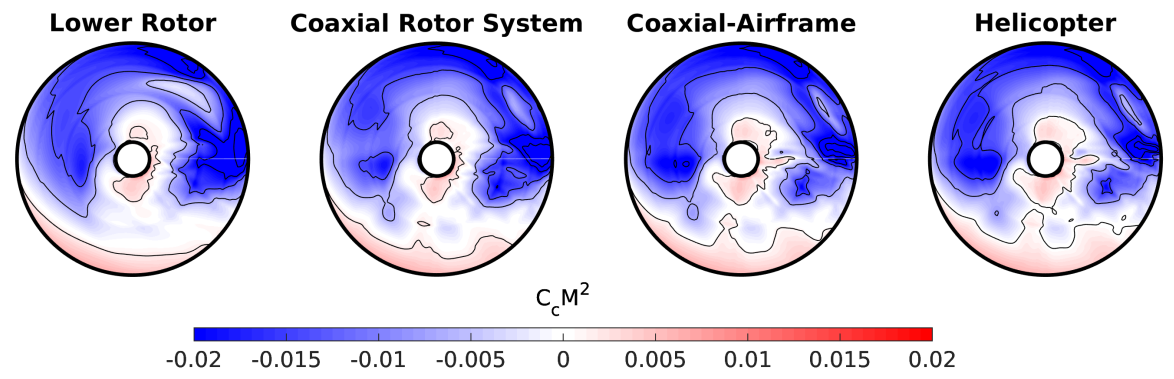

(b) Chordwise force

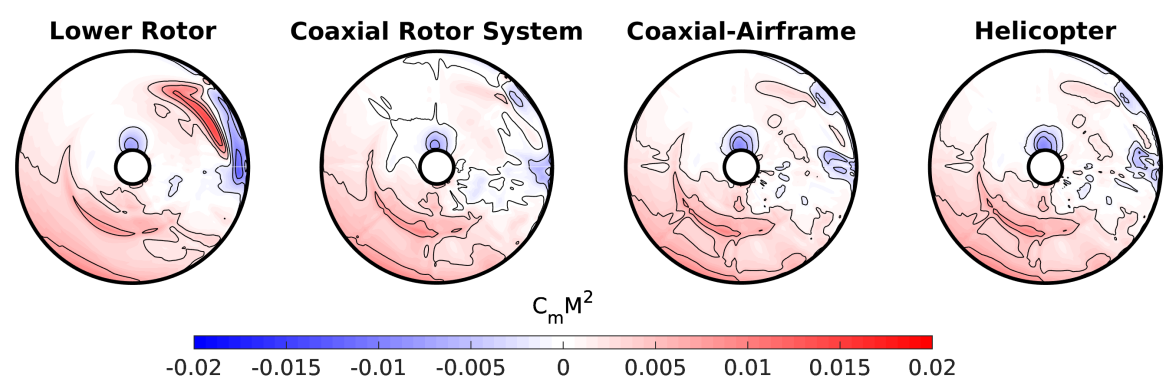

(c) Pitching moment

Figure 8.5: Aerodynamic interactions effects on the lower rotor at 150 knots. 


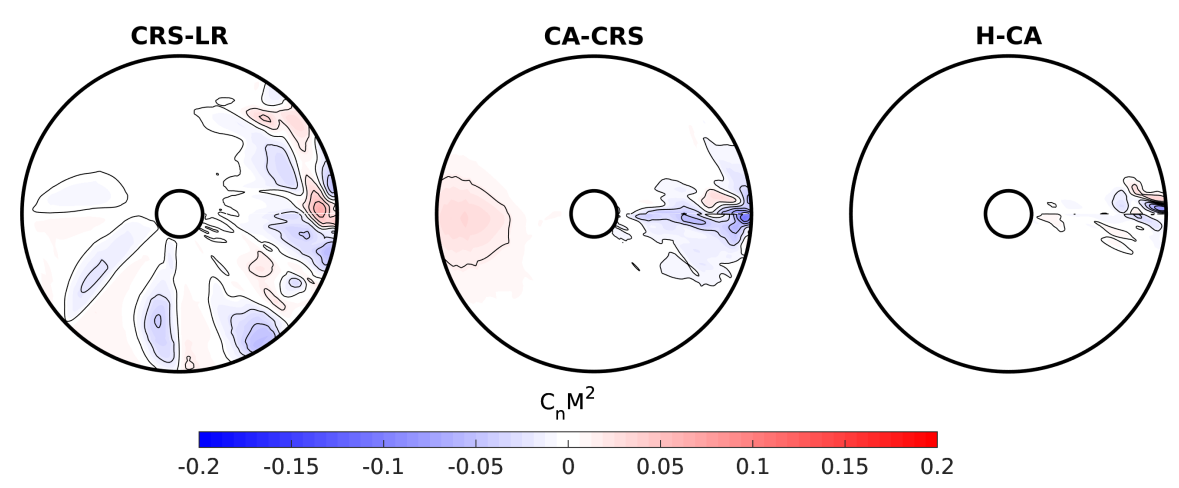

(a) Normal force

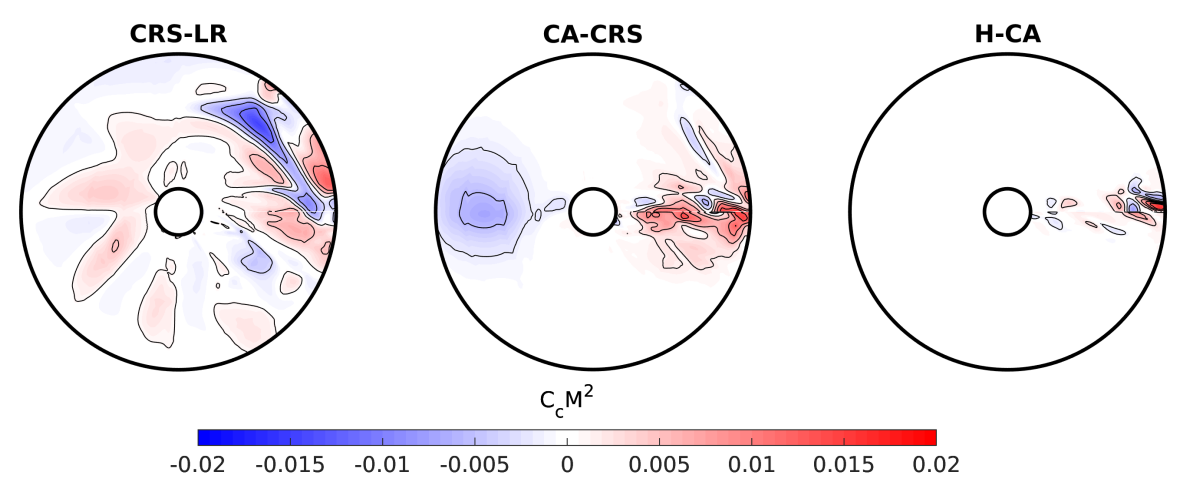

(b) Chordwise force

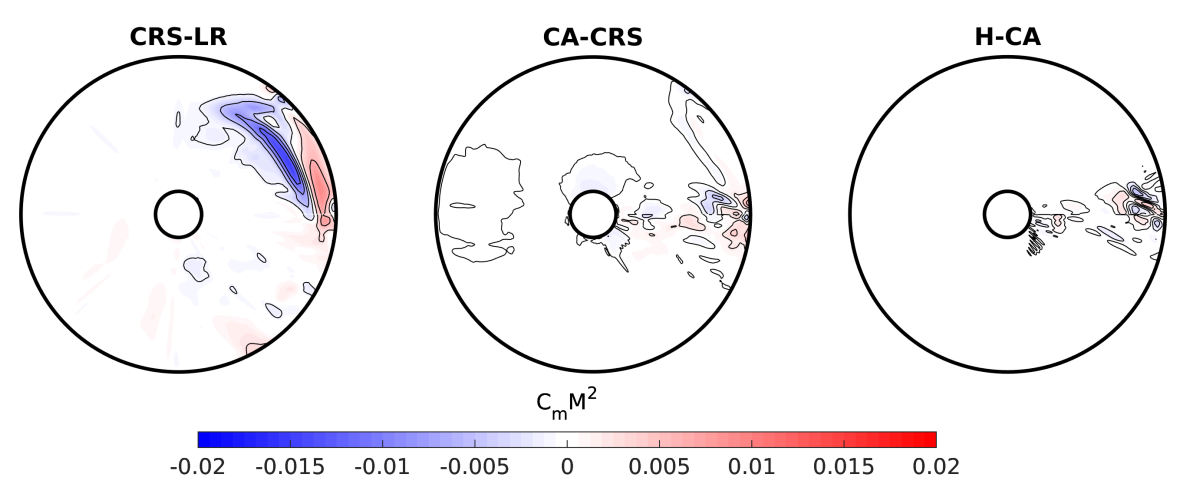

(c) Pitching moment

Figure 8.6: The difference between the coaxial rotor system and the lower rotor, the coaxial airframe combination and coaxial rotor system, helicopter and coaxial airframe combination at 150 knots. 


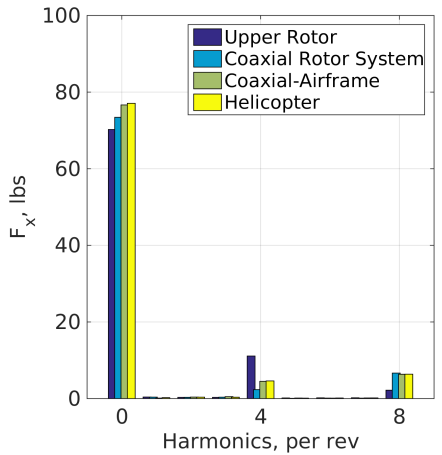

(a)

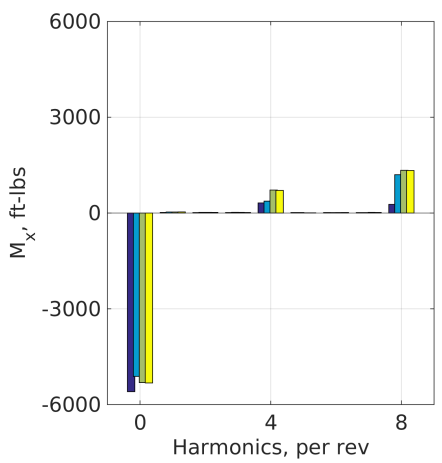

(d)

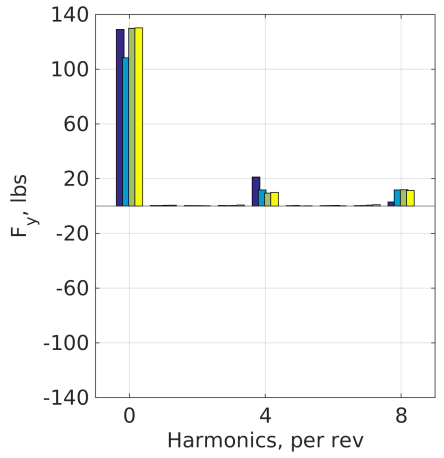

(b)

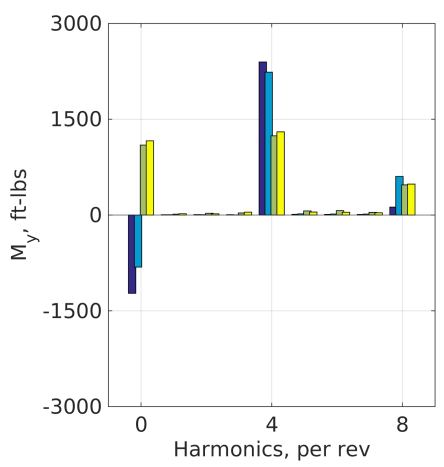

(e)

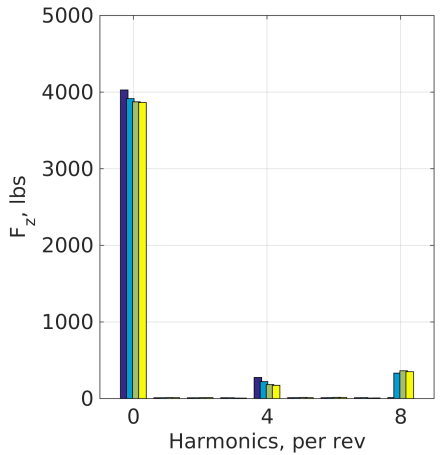

(c)

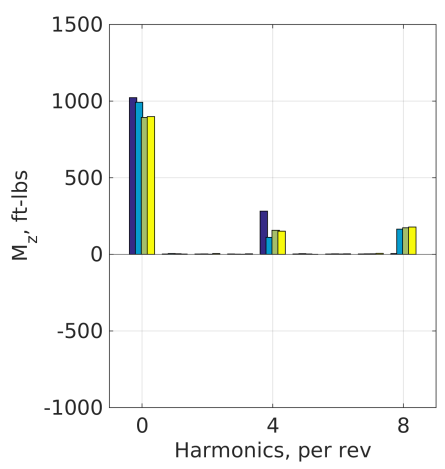

(f)

Figure 8.7: Lower rotor hubloads for various configurations shown in Fig. 8.5(a) at 150 knots. 


\subsection{Interactional Aerodynamics Affecting the Airframe}

The objective of this section is to examine the interactional aerodynamic effects on the airframe resulting from the coaxial rotor and the propeller, at a flight speed of 150 knots. The CFD airframe unstructured grid consists of smooth fuselage, pylon, mast, vertical stabilizer and two horizontal stabilizers pitched at positive 5 degrees to the horizontal. The rotor hubs are not modeled in this work. The cases considered in this study: (a) isolated airframe, (b) airframe + coaxial rotor, and (c) full aircraft. The aerodynamic forces and moments are in the global CFD frame of reference, centered at the lower hub. The axial force, $F_{x}$, is positive towards the tail of the aircraft. The side force, $F_{y}$, is positive starboard. The vertical force, $F_{z}$, is positive up.

Figure 8.9(a) shows the time-history of the airframe aerodynamic axial force, from CFD simulations, with respect to azimuth angle in a rotor reference frame. The presence the coaxial rotor system in the CFD introduces a periodic increase in spanwise force every 90 degrees. The periodic increase in spanwise force occurs when the rotor blades cross over the airframe. Figure 8.9(b)shows the harmonic amplitudes of the spanwise force up to $8 \mathrm{P}$, the $4 \mathrm{P}$ blade passage frequency is dominant. Figures 8.9 (d) and (e) show the harmonic contributions from various airframe components, with the fuselage being the main source of the $4 \mathrm{P}$ and $8 \mathrm{P}$ harmonic contributions. Figure 8.9 (c) shows the mean spanwise force contributions from various airframe components. For an isolated airframe, fuselage and mast were the main contributers of spanwise force. The cylindrical mast is a bluff body which produces 
significant amount of pressure drag. The presence of the rotor increased the fuselage spanwise force. The addition of the propeller increased the airframe spanwise force.

Figure 8.10 (a) shows the time-history of the airframe aerodynamic side force for various configurations. The airframe in isolation experiences periodic fluctuations that stem from the shedding off of the pylon and the mast. The presence of the rotor induces additional frequencies and an increase in the mean of side force. Figure 8.10(b) shows the harmonic amplitudes of the side force of the airframe. For an isolated airframe, the $6 \mathrm{P}$ is dominant. The addition of the rotor and the auxiliary propeller introduced strong $4 \mathrm{P}$ and $8 \mathrm{P}$. The fuselage and the pylon are the main components that contributed to the $4 \mathrm{P}$ response, blade passage frequency. Figure 8.10(c) shows the steady side force contributions from various aircraft components for the three configurations. There is an increase in the airframe side force in the presence of the rotor and the auxiliary propeller. For an isolated airframe the horizontal tails, produce roughly equal but opposite side force. The side force results from the roll up and shedding of the tip vortices from the horizontal tail surfaces. The presence of the coaxial rotor significantly reduces the side force on the port side horizontal tail. The starboard horizontal tail side force is also reduced but not as much as the port side, resulting in an imbalance The vertical tail also sees a non-zero side force.

Figure 8.11(a) shows the time-history of the airframe aerodynamic vertical force for configurations (a) through (c). The presence of the rotor introduces significant loading peaks every 90 degrees, which are due to blade passage. Figure 8.11(b) quantifies the frequency of the loading peaks observed in Fig.8.11(a) to be 
4P (dominant) and 8P. Figures 8.11(d) and (e) show that the $4 \mathrm{P}$ and $8 \mathrm{P}$ airframe forcing comes from the fuselage and the horizontal tails. The steady normal force for various airframe components, averaged over one rotor revolution, is shown in Fig. 8.11 (c). In the presence of the rotor there is a negative download on the fuselage. As the blades passover the airframe, the pressure on the top surface of the fuselage increases as shown in Fig 8.8 , creating a downward force on the fuselage. Figure 8.11 (c) also shows that the pylon and mast are creating upward normal force. There is some negative pressure on the top of the pylon and the mast, which may enable these parts to act as lifting surfaces.

The time-history of the airframe aerodynamic roll moment about the center of the lower rotor hub is shown Figure 8.11(a) for configurations (a) through (c). For an isolated airframe, there are low amplitude periodic fluctuations roughly about the zero mean. An introduction of the rotor into the simulations results in higher, nonzero, mean as well as additional frequency content. The presence of the auxiliary propeller does not have significant effect on the mean or fluctuations of the roll moment. Figure 8.12 (b) compares the harmonic amplitudes of the roll moment for various configurations. For an isolated airframe $6 \mathrm{P}$ harmonic dominates, whereas $4 \mathrm{P}$ and $8 \mathrm{P}$ harmonics dominate for the cases with coaxial rotor. The $6 \mathrm{P}$ is the shedding frequency of the mast. The $4 \mathrm{P}$ is the blade passage frequency over the airframe. The fuselage and the horizontal tails are the key airframe components that produce the $4 \mathrm{P}$ and $8 \mathrm{P}$ roll moment. The total steady roll moment on the isolated airframe is roughly zero as shown in Fig 8.12 (c), equal and opposite roll moment is produced by the horizontal tails without additional significant contributions from 
other airframe components. However, in the presence of the rotor there is a positive roll moment (starboard down) on the aircraft, with contributions from fuselage, horizontal tails and vertical tail. The non-zero roll moment on the airframe is a result of interactional aerodynamics and is an important factor to consider when trimming the vehicle.

Figure 8.13 (a) shows the time-history of the airframe aerodynamic pitching moment about the center of the lower rotor hub. The pitching moment is positive when the vehicle is pitching nose up. An isolated airframe experiences a relatively steady, nose down pitching moment. The majority of the pitching moment is produced by the horizontal tail surfaces, shown in Fig 8.13 (c). In the presence of the rotor, the airframe experiences fluctuations in the pitching moment which arise from interactional aerodynamics. The pitching moment harmonic amplitudes are shown in Fig 8.13 (b). The dominant harmonic is the $4 \mathrm{P}$. An $8 \mathrm{P}$ is present but it's amplitude is roughly half of the $4 \mathrm{P}$. The $4 \mathrm{P}$ harmonic stems from the fuselage, shown in Fig $8.13(\mathrm{~d})$, due to the blades passing directly over the fuselage. The $8 \mathrm{P}$ harmonic is due to a combination of the horizontal tails and the fuselage, shown in 8.13(e).

The time-history of the aerodynamic airframe yaw moment, positive counterclockwise, about the center of the lower rotor hub is shown in Fig.8.14(a). An isolated airframe sees a roughly steady yaw moment centered at zero. For an isolated airframe, the horizontal tails produce equal and opposite yaw moments, shown in Fig 8.14 (c). The air flows from root to tip on the horizontal tails, creating an equal and opposite radial force. In the presence of the rotor, the starboard horizontal tail produces more yaw moment compared to the port horizontal tail. Compared to the 
isolated airframe, where there was no significant yaw moment contributions from other airframe components, in the presence of the rotor additional yaw moment is produced by the vertical tail and the fuselage. In addition, in the presence of the rotor, the airframe experiences a significant $4 \mathrm{P}$ yaw moment as well as a $8 \mathrm{P}$, shown in Fig 8.14(b), due to the interactional aerodynamics on the fuselage, 8.14(d) and (e).

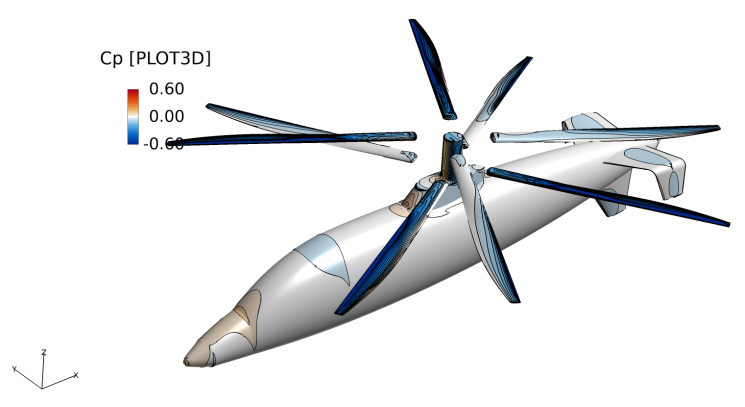

(a)

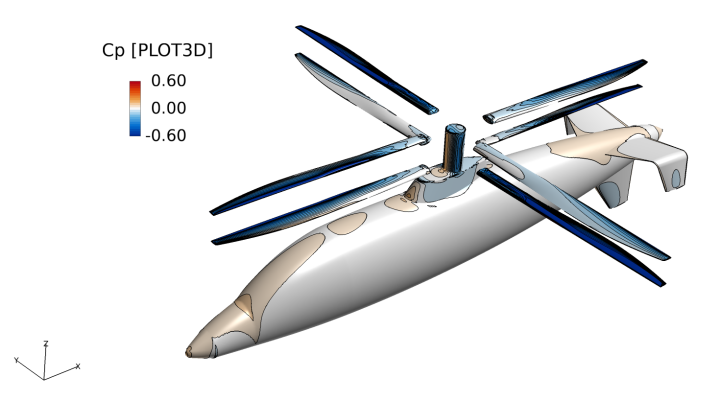

(b)

Figure 8.8: Effect of blade passage on the pressure over the airframe 150 knots. 

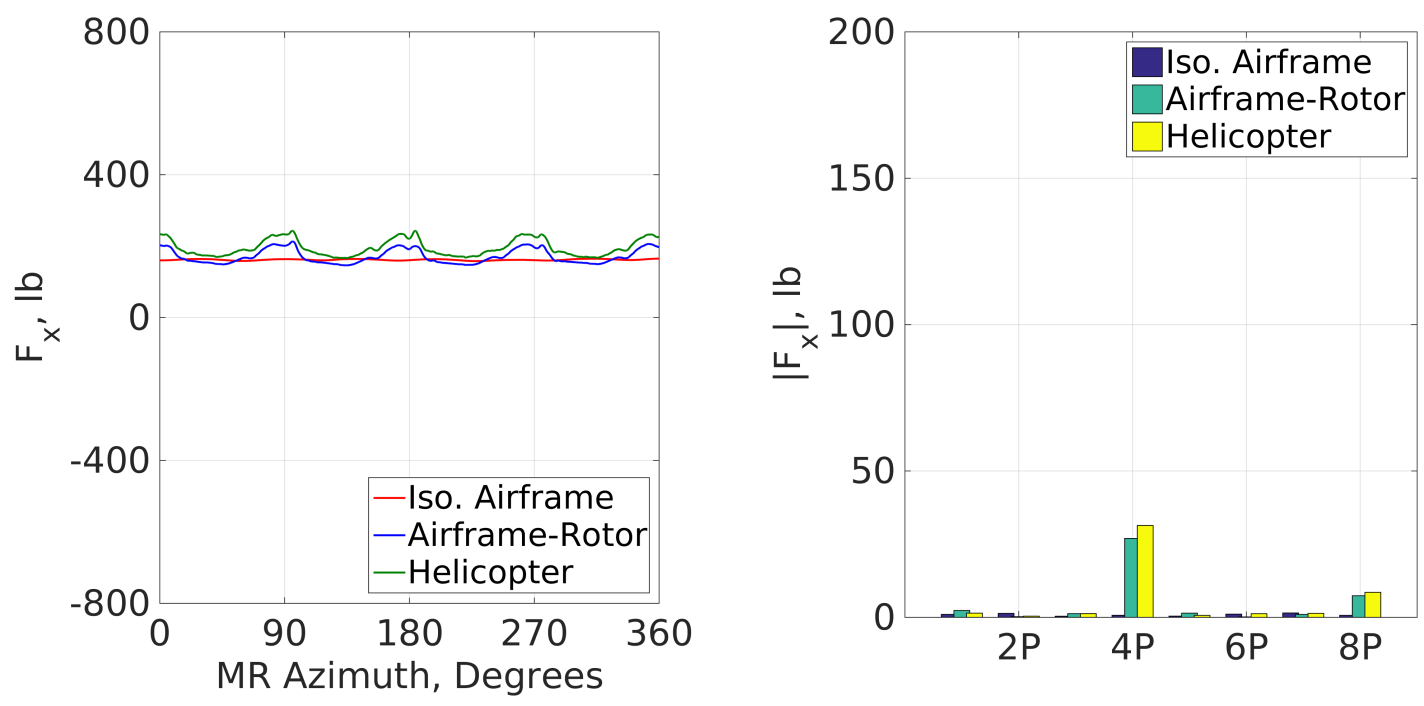

(a)

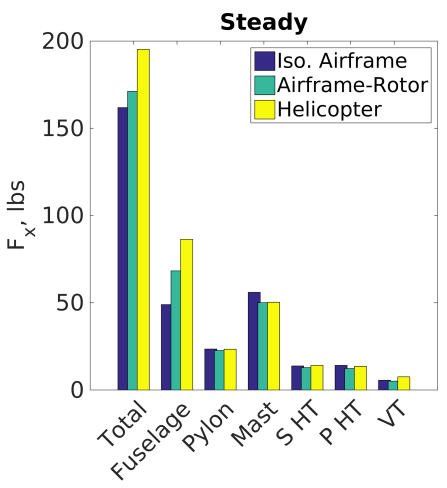

(c)

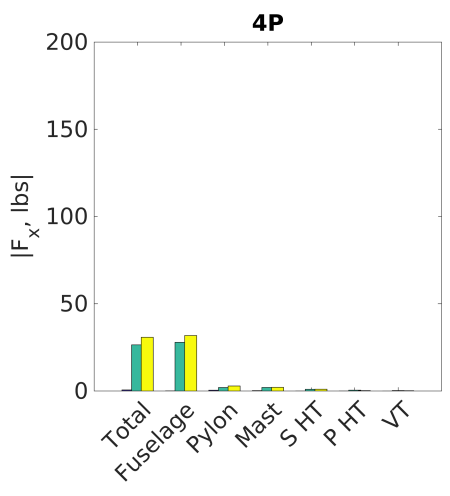

(d) (b)

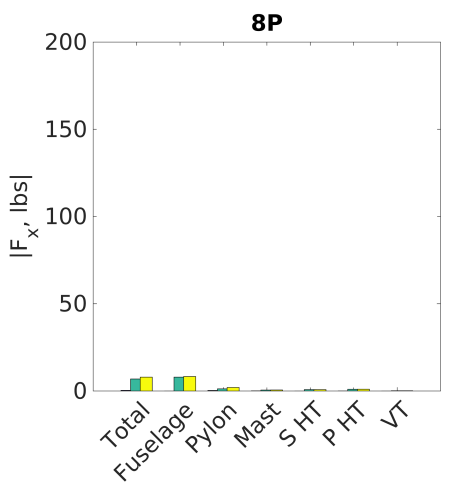

(e)

Figure 8.9: The time-series and harmonic amplitudes of the airframe spanwise at 150 knots. 

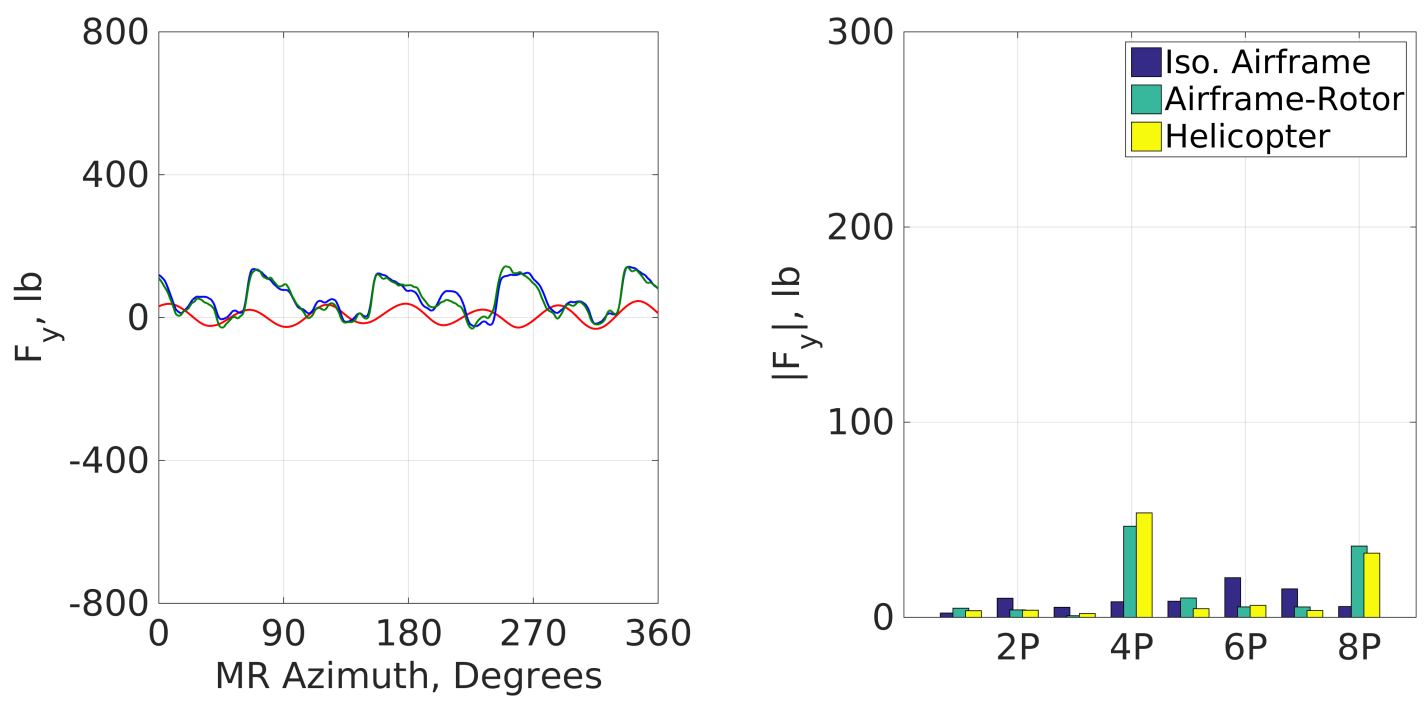

(a)

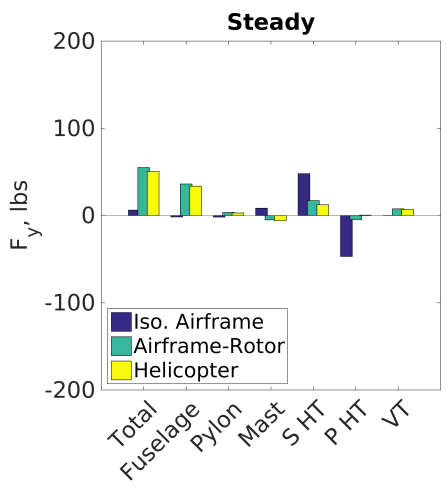

(c)

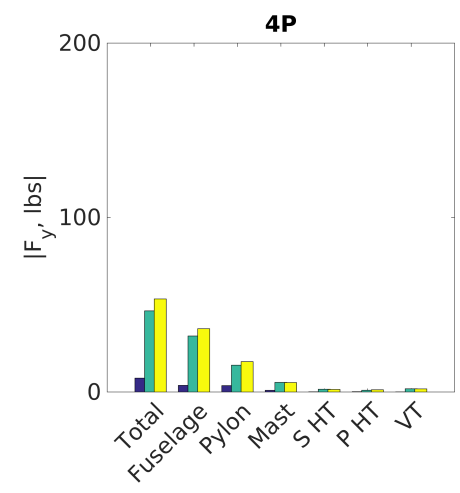

(d) (b)

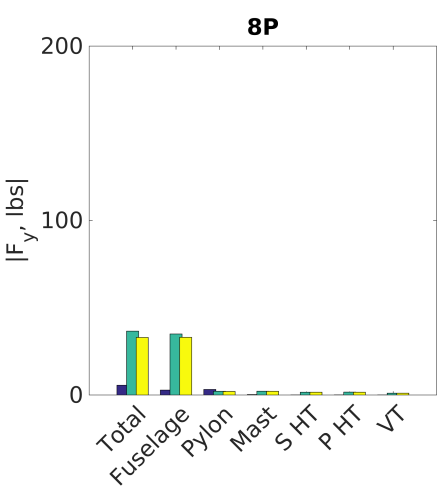

(e)

Figure 8.10: The time-series and harmonic amplitudes of the airframe side force at 150 knots. 

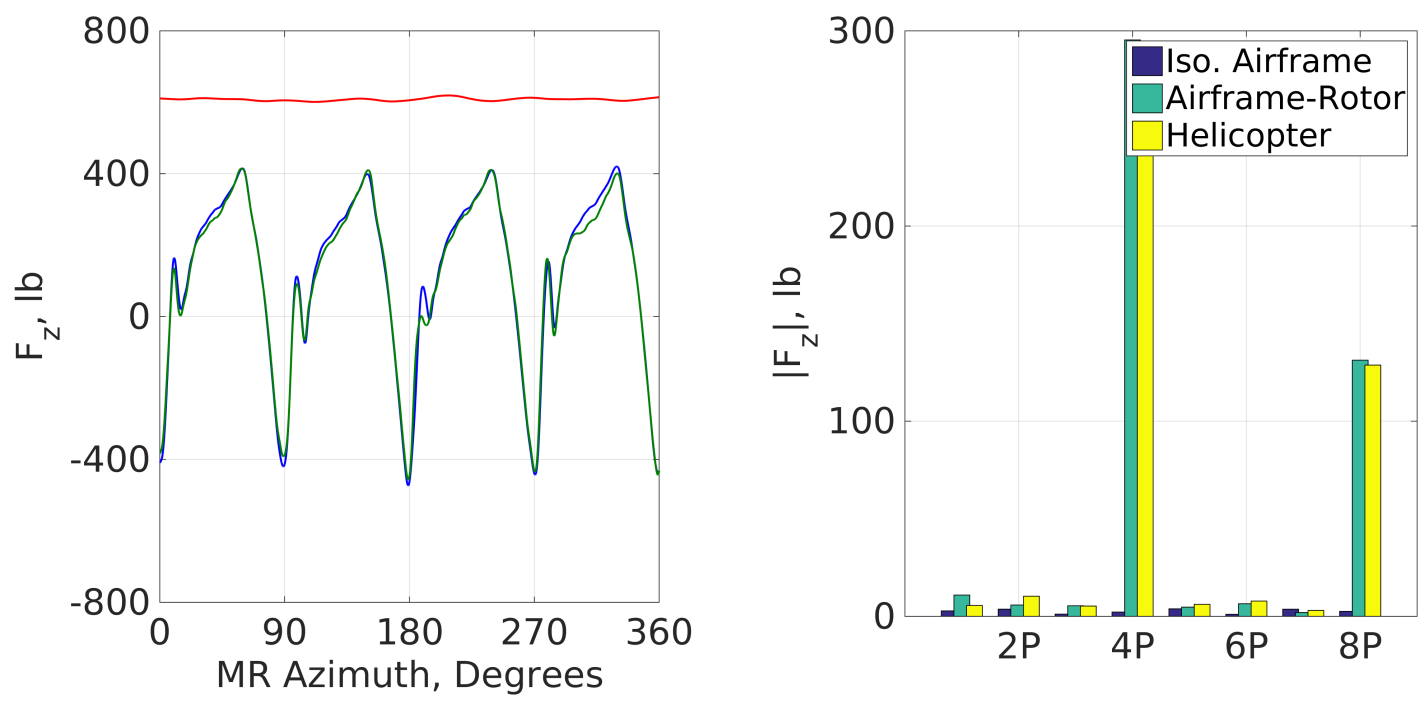

(a)

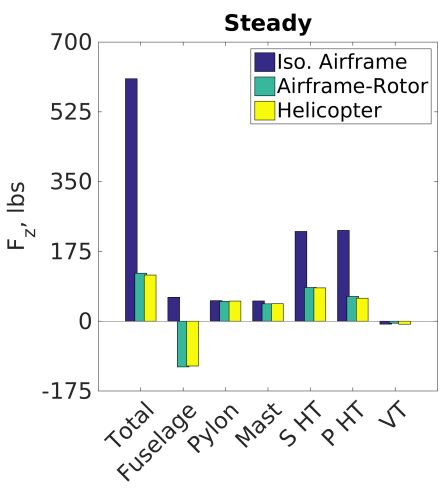

(c)

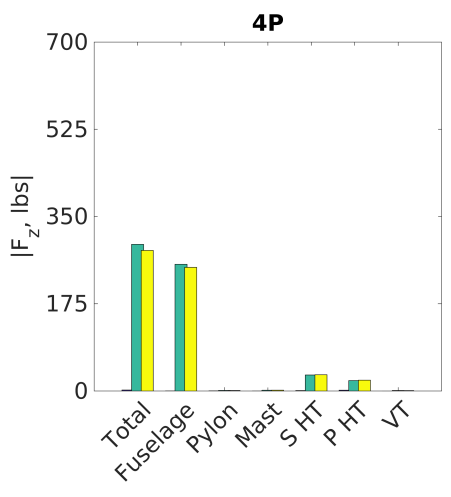

(d) (b)

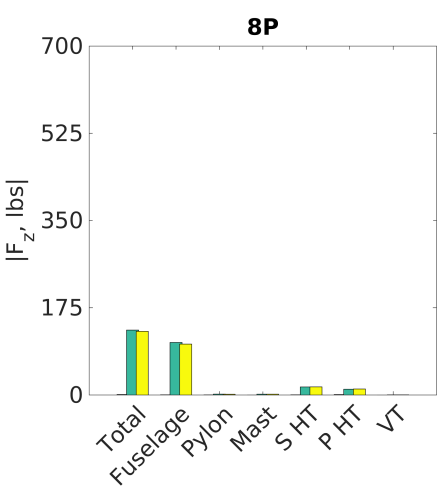

(e)

Figure 8.11: The time-series and harmonic amplitudes of the airframe normal force at 150 knots. 

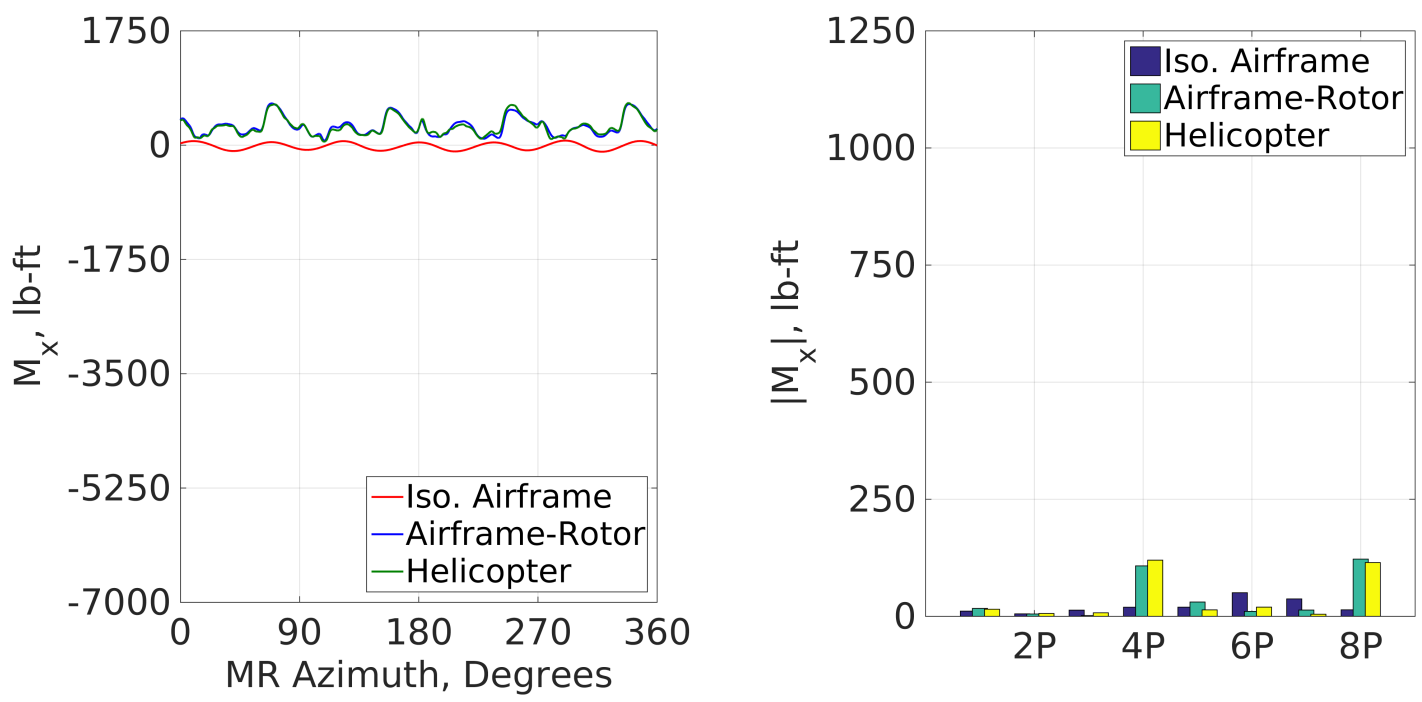

(a)

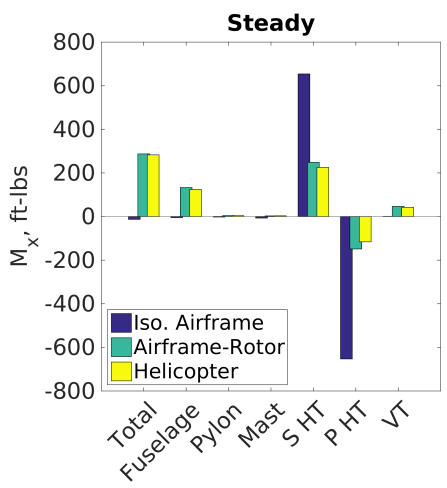

(c)

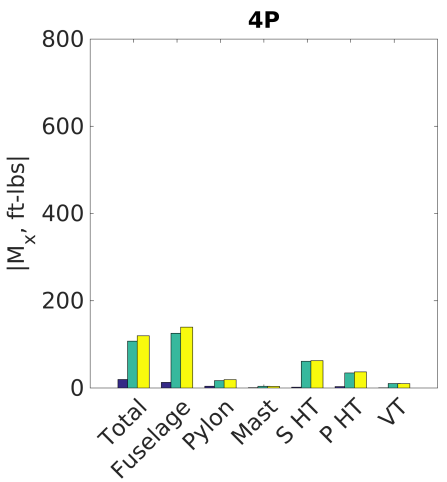

(d) (b)

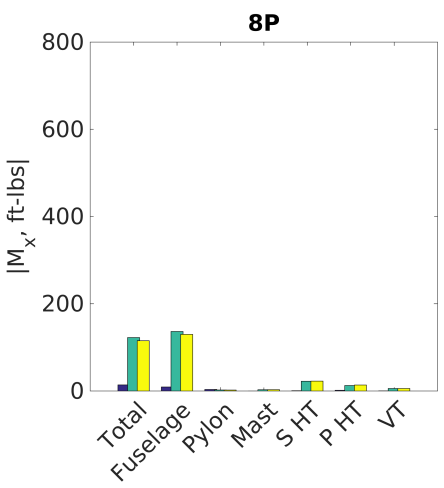

(e)

Figure 8.12: The time-series and harmonic amplitudes of the airframe roll moment, positive starboard side up, about the center of lower hub at 150 knots. 

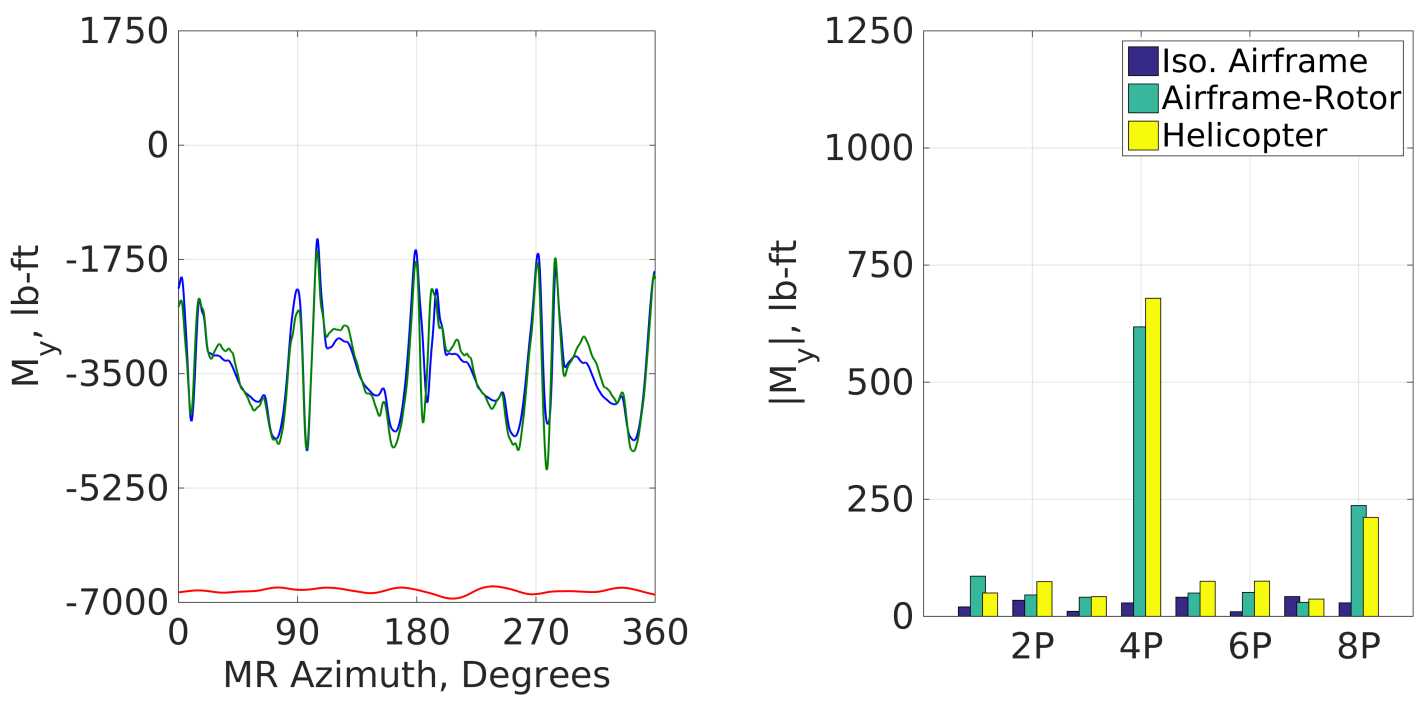

(a)

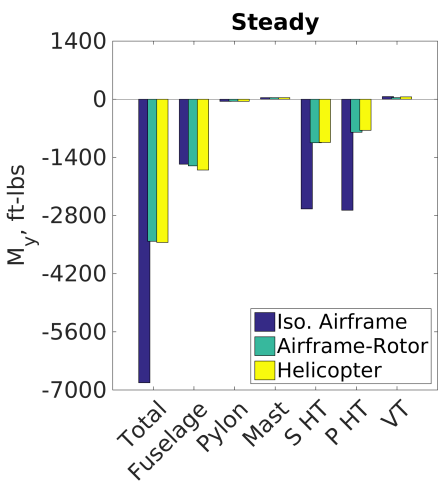

(c)

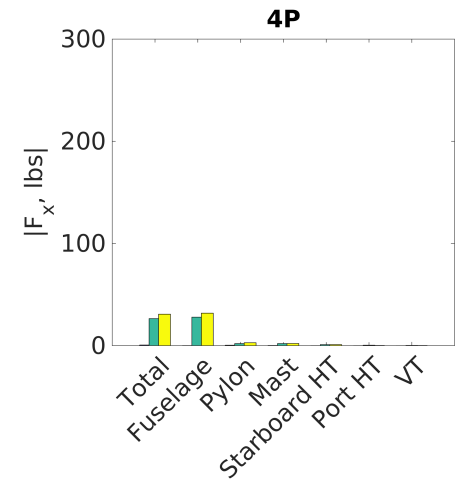

(d) (b)

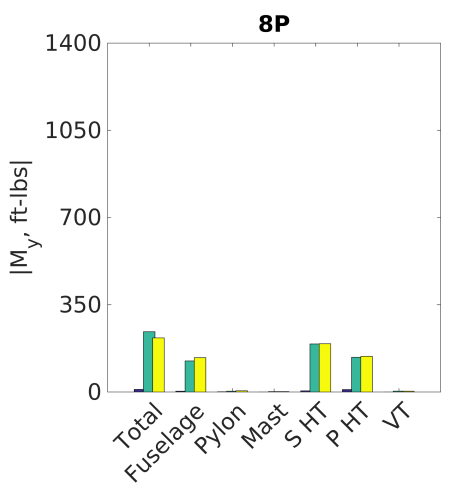

(e)

Figure 8.13: The time-series and harmonic amplitudes of the airframe pitching moment, positive nose up, about the center of lower hub at 150 knots. 

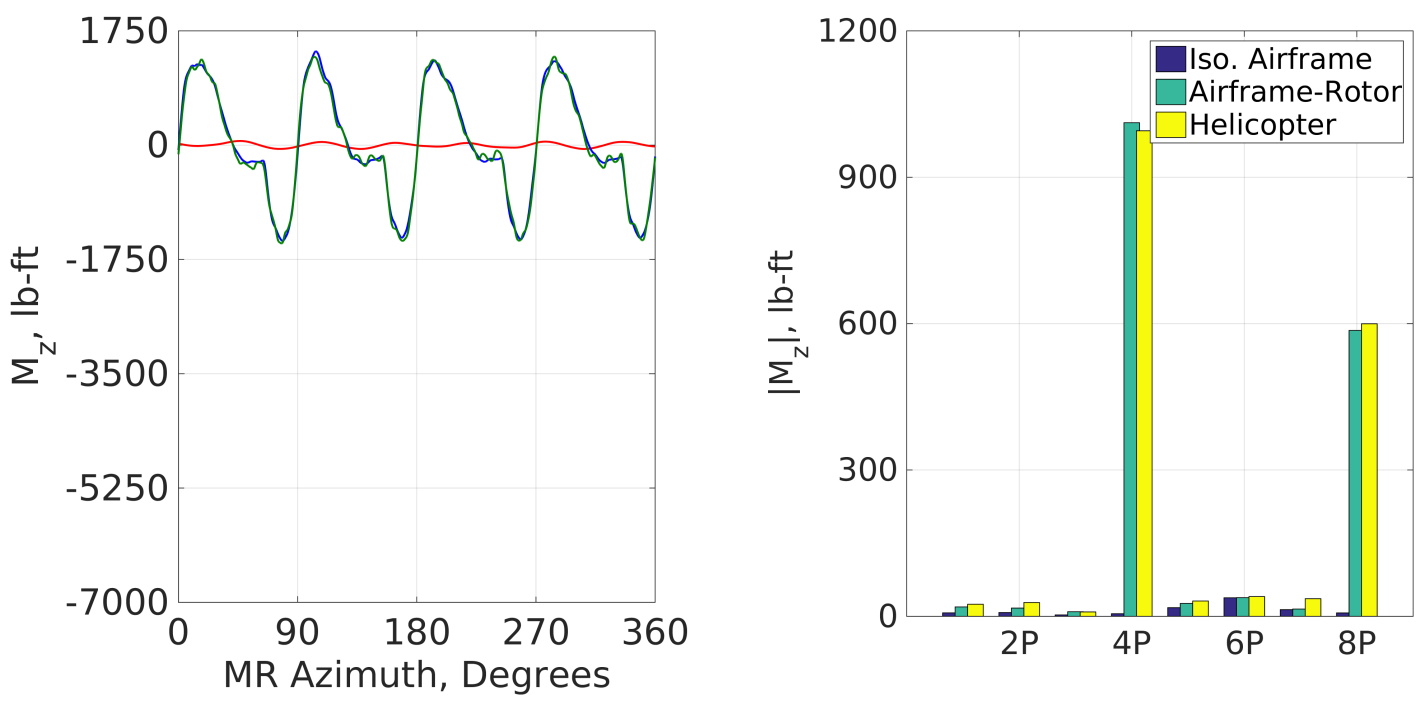

(a)
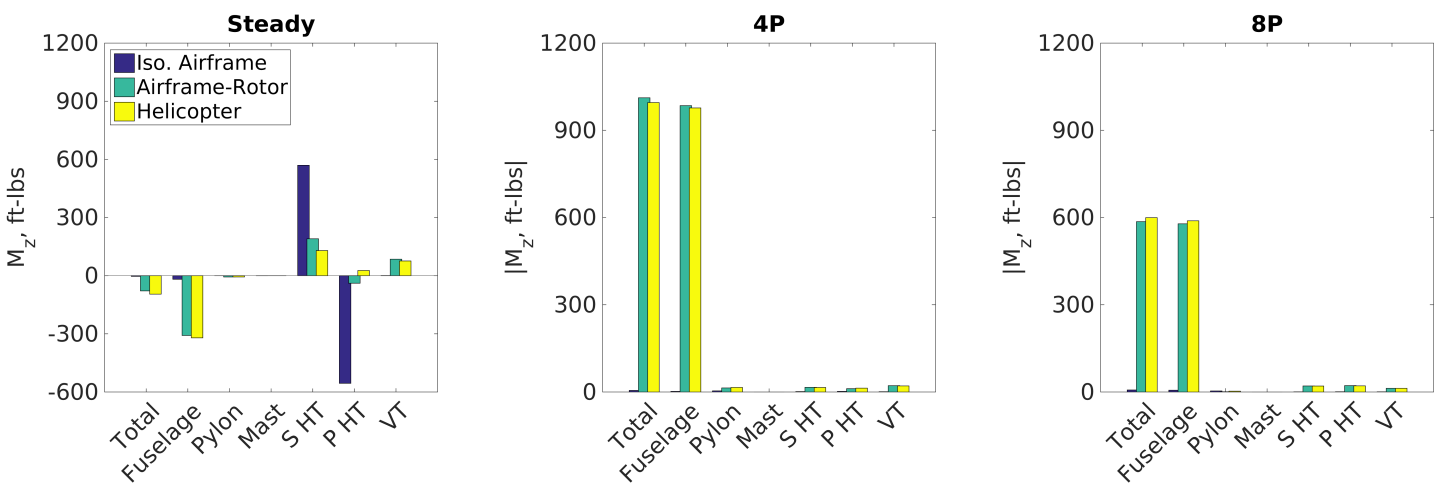

(c)

(d)

(e)

Figure 8.14: The time-series and harmonic amplitudes of the airframe yaw moment, positive counter-clockwise, about the center of lower hub at 150 knots. 


\subsection{Interactional Aerodynamics Affecting the Auxiliary Propeller}

This section examines the effects of helicopter components on the performance of the auxiliary propeller for the vehicle traveling at 150 knots. The collective of the auxiliary propeller blades was obtained from the converged full vehicle CFD/CSD coupling discussed in Chapter 7. The performance of the auxiliary propeller is analyzed for three configurations shown in Fig 8.15(a); isolated propeller, propeller airframe combination and helicopter configuration. The auxiliary propeller is rotating counter-clockwise, with $\psi=0$ degrees at the top and $\psi=90$ degrees on the starboard side.

Figure 8.15(b) shows the azimuthal distribution of the propeller normal force for the configurations shown in Fig 8.15(a). A propeller in isolation experiences smooth normal force distribution over the propeller disk, with advancing side producing less normal force than the retreating side. The imbalance in the normal force is a consequence of the vehicle pitch attitude. At a flight speed of 150 knots, the vehicle is trimmed nose up. Instead of flow coming in normal to the propeller disk, the flow comes in at an angle. On the advancing side of the rotor disk, the edgewise velocity at the propeller disk is subtracted from the rotational velocity, because of the positive pitch attitude tilt. On the retreating side of the rotor disk, the edgewise velocity at the propeller disk is compounded with the rotational velocity, resulting in a higher normal force. The presence of the airframe increases the normal force on the inboard stations. On an isolated propeller, the angle of attack on the inboard stations of the blades is quite low due to the high inflow velocity, which is roughly 
Table 8.2: Mean auxiliary propeller thrust and power for three configurations at 150 knots.

\begin{tabular}{l|c|c|c}
\hline \hline & Iso. Prop & Prop-Airframe & Helicopter \\
\hline \hline Thrust (lb) & 468.8 & 676.1 & 697.5 \\
\hline Power (hp) & 293.8 & 393.5 & 400.7 \\
\hline
\end{tabular}

the same as the forward flight speed of the vehicle. The wake from the airframe reduces the inflow velocity at the rotor disk, increasing the sectional angle of attack on the inboard stations of the propeller, and as a consequence, the normal force. It is also observed that the normal force on the outer sections of the rotor increases in the presence of the airframe, especially near $\psi=0, \psi=90, \psi=180$ and $\psi=270$ degrees, as shown in Fig $8.15(\mathrm{~b})$. At these locations, there are strong interactions between the propeller and the wake from the mast (near $\psi=0$ degrees), horizontal tails $(\psi=90$ and $\psi=270$ degrees $)$ and the vertical tail $(\psi=180$ degrees $)$. The increase in normal force and as a result the efficiency of the propeller, is a consequence of a phenomena known as boundary-layer-ingestion, which has been studied extensively by Min et al. in Ref. [86]. This phenomena is further explored by performing a collective sweep using CFD simulations, for an isolated propeller and airframe-propeller combination, shown in Fig D.1. 


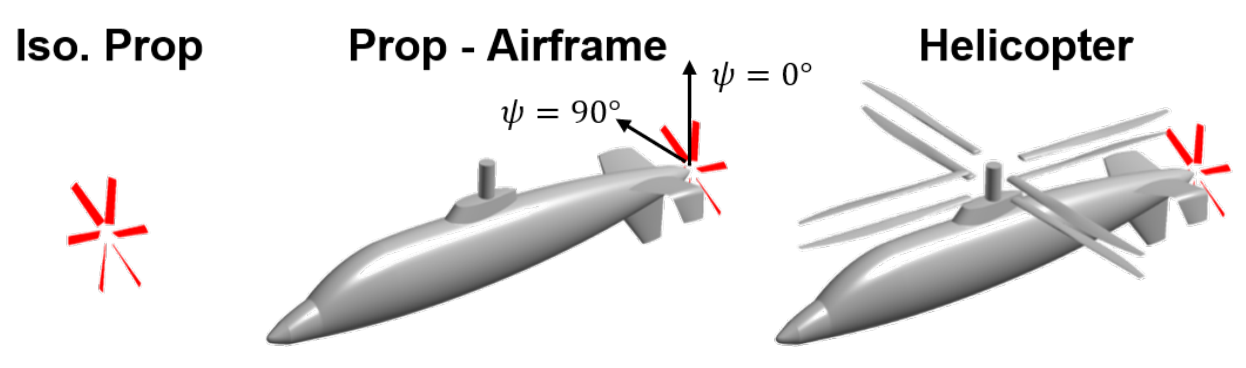

(a) Configurations

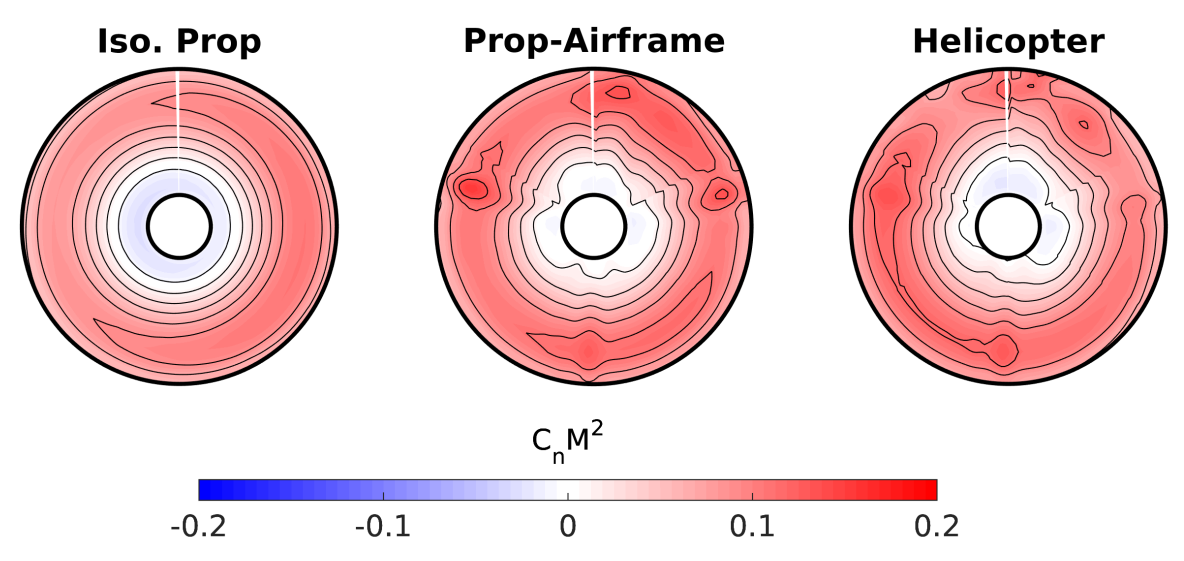

(b) Auxiliary propeller normal force.
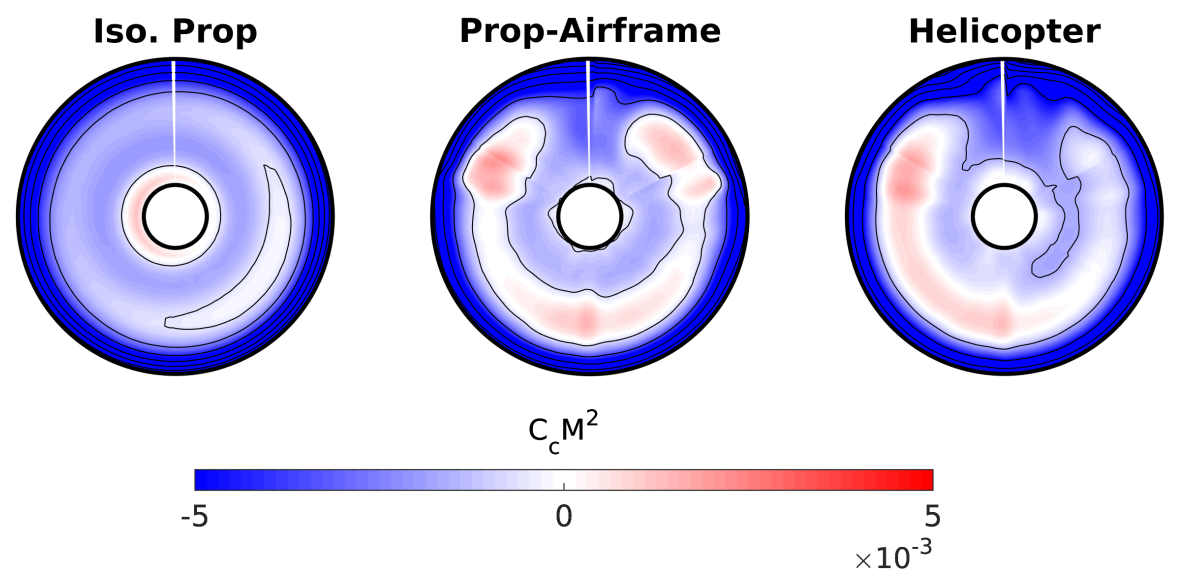

(c) Auxiliary propeller chordwise force.

Figure 8.15: Auxiliary propeller airloads at 150 knots. 


\section{Conclusions}

\subsection{Summary of Research}

The future multi-role aircraft designs consist of various complex components that aerodynamically interact with one another and each other's wakes. The aerodynamic interactions may result in additional vibrations, as well as degradation of the performance. Often times it is necessary to redesign the components as well as their placement on the aircraft post-production. To avoid the costly redesign, it is of paramount importance to fundamentally understand the source of the interactions as well as their implications on the performance prior to production of the aircraft.

This work presented a numerical study of the fundamental aerodynamic interactions between the components of a high-speed compound coaxial helicopter using conventional and full vehicle CFD/CSD coupling approach. A high-speed compound coaxial helicopter, notional X2TD, consisting of a coaxial rotor, airframe and an aft-mounted propeller, was simulated in this study. A high-fidelity CFD framework, HPCMP CREATE ${ }^{T M}$-AV Helios, was used in conjunction with an in-house CSD solver, to simulate helicopter in forward flight.

The results shown in this thesis are presented in three parts. The first part of the results, discussed in Chapter 6 , focused on studying the trends for a coaxial rotor 
at a range of advance ratio, $\mu=0.14$ to $\mu=0.65$. A conventional rotor $\mathrm{CFD} / \mathrm{CSD}$ coupling methodology, where only the rotor airloads are transferred from the CFD solver to the CSD solver, was utilized for the first part of the results. The CFD simulations for this part of the results only included the coaxial rotor system. The second part of the results, discussed in Chapter 7, focused on analyzing the effect of the full vehicle CFD/CSD coupling on the trim parameters and airloads. The CFD simulations included the coaxial rotor system, the airframe and auxiliary propulsor. For this results section, the coaxial rotor airloads, time-averaged airframe airloads and the pusher propeller thrust and power were transferred from the CFD solver to the CSD solver. The final part of the results, presented in Chapter 8, focused on the fundamental understanding of interactional aerodynamics of a high-speed compound helicopter. The CFD simulations of components in isolation, in combination and in full configuration were performed. The unsteady coaxial rotor airloads were compared for different configurations. The auxiliary propeller performance was compared for an isolated as well as an installed propeller.

\subsection{Key Conclusions}

This section presents the key conclusions obtained from the results given in Chapters 6, 7 and 8, Each chapter contains an in depth analysis and discussion of the conclusions. The key conclusions are addressed in order, corresponding to their respective chapters. 


\subsubsection{Coaxial Rotor CFD/CSD Coupling}

This subsection summarizes the key results from Chapter 6. The results presented in Chapter 6 focused on characterizing the trim solution, airloads and structural deformation for a lift-offset coaxial rotor at five advance ratios ranging between $\mu=0.14$ and $\mu=0.65$ (50 knots and 225 knots). For these results, in Chapter 6 , only the coaxial rotor was present in the CFD simulations. A conventional CFD/CSD coupling approach was utilized to correct the comprehensive analysis airloads.

A significant difference was observed between the upper and lower rotor collectives at 50 knots and 225 knots, but not at other speeds. The lower rotor collective was $3 \%$ higher compared to the upper rotor collective for the 50 knot forward flight condition. At 250 knots, the upper rotor collective was higher than the lower collective by roughly $7 \%$. At the intermediate speeds, the upper and lower rotor collectives were within $0.6 \%$ of each other. For the 50 knot case, the combination of the relatively low flight speed and the forward tilt of the rotor disks resulted in the upper rotor wakes passing through the lower rotor. This caused a decrease in the sectional angle of attack and the mean thrust of the lower rotor, which resulted in the lower rotor trimming to a higher collective. To verify this phenomena a CFD/CSD test case was run with the upper and the lower in isolation in CFD. This test case showed that without the rotor-rotor interference, both rotors trim to a roughly identical collectives.

The advance ratio sweep showed a significant increase in the negative loading on the outboard stations of the rotor blade, on the advancing side. For 225 knots, 
roughly $40 \%$ of the blade was negatively loaded at $\psi=45$ degrees azimuth. This was due to the decrease in angle of attack on the outboard stations of the blade on the advancing side.

The advance ratio sweep also showed that there is a pronounced redistribution of the normal force over the rotor disk. At lower speeds the normal force was equally distributed around the rotor disk. However, as the speed increased the fore and aft portions of the rotor disk carried the majority of the normal force. This was a result of the development of the negative loading on the advancing side, first quadrant, at the outboard regions of the blade.

At low speeds a distinct $8 \mathrm{P}$ impulsive loading was observed every quarter revolution due to blade-blade interactions. As the forward flight speed increased, a decrease in the magnitude of the $8 \mathrm{P}$ blade-blade impulsive loading spikes on the advancing side and an increase in magnitude of the $8 \mathrm{P}$ blade-blade loading spikes on the retreating side was observed.

\subsubsection{Results Part II: Vehicle CFD/CSD Coupling}

This subsection summarizes the key findings from the full vehicle CFD/CSD coupling study, presented in Chapter 7. The objective of Chapter 7 was to show the effect of incorporating the airframe and propeller into the CFD/CSD coupling methodology. The results from the full vehicle CFD/CSD coupling approach were compared to those obtained with the rotor CFD/CSD coupling methodology. The final trim solution, the main rotor airloads and the main rotor blade deformations 
were compared.

The study showed that correcting CSD low order airframe airloads with highfidelity CFD airloads changed the final trim state of the vehicle. The CSD low order aerodynamic model assumed zero roll and yaw moment on the fuselage. The presence of the rotor above the airframe resulted in the nonzero airframe yaw and roll moments, affecting the final trim state of the rotor. For 150 knots, the rotor CFD/CSD coupling procedure predicted very similar upper and lower rotor collectives. However, when performing the full vehicle CFD/CSD coupling procedure the lower rotor collective increased by $16 \%$, while the upper rotor collective decreased by $4 \%$. In addition, the presence of the airframe under the rotor system affected the airloads on the main rotor which in turn also affected the final rotor trim solution.

Performing the full vehicle CFD/CSD coupling procedure also enabled a highfidelity prediction of the propeller thrust, accounting for the boundary-layer-ingestion and rotor wake effects. The propeller thrust in the comprehensive analysis look-up table was derived using simplified expression, $P=T V$, from momentum theory. Correcting the table look-up propeller thrust, derived from simplified expression $P=T V$, with high-fidelity $\mathrm{CFD}$, also affected the final vehicle pitch attitude. The rotor CFD/CSD coupling predicted a vehicle pitch attitude of 2 degrees, while the full vehicle CFD/CSD coupling predicted a 2.8 degree pitch attitude. 


\subsubsection{Results Part III: Fundamental Understanding of Interactional Aerodynamics of Compound Coaxial Helicopter}

This subsection summarizes the key results from Chapter 8. The objective of Chapter 8 was to identify the aerodynamic interactions between various components of the notional coaxial compound helicopter and determine their effect on the performance and the vibratory airloads at 150 knots. The CFD simulations were performed with individual components and component combination.

The isolated upper and lower rotors experienced relatively smooth airloads distribution. However, the presence of the lower rotor under the upper rotor introduced a significant $8 \mathrm{P}$ loading, detectable as an $8 \mathrm{P}$ loss in normal force every 45 degrees. The magnitude of the $8 \mathrm{P}$ hub vertical force increased by $90 \%$ in the presence of the lower rotor, resulting from blades of the upper rotor crossing with the blades of the lower rotor. The $8 \mathrm{P}$ hub roll moment increased by $85 \%$.

The presence of the rotor above the airframe, introduced significant $4 \mathrm{P}$ and $8 \mathrm{P}$ download, due to blade passage over the fuselage. Both, the magnitude of the $4 \mathrm{P}$ and $8 \mathrm{P}$ harmonics went up by roughly $100 \%$. The presence of the propeller did not have a significant effect on the airframe airloads.

The boundary-layer-ingestion effect increased the efficiency of the propeller in an installed configuration. An isolated propeller produced a thrust equivalent 468 pounds, while an installed propeller produced a thrust of 698 pounds. As expected, the required power also increased for an installed configuration. However, the overall efficiency of the propeller increased. It was also observed that the rotor wake effected 
the unsteady airloads on the propeller, increasing the normal force on the midspan of the advancing side, while decreasing the normal force on the retreating side.

\subsection{Recommendations for Future Work}

This section provides the reader with suggestions for additional work which may include but is not limited to the following items:

1. Expanding the simulated flight conditions to investigate the effect of advance ratio on the interactional aerodynamics between the helicopter components. The current study focused on understanding the interactional aerodynamics of a helicopter at a cruise speed of 150 knots.

2. Expanding the simulated flight conditions for the rotor CFD/CSD study to include speeds up to or beyond 250 knots. The future vertical lift rotorcraft configurations promised to achieve speeds greater than 250 knots, therefore simulating and understanding high-speed coaxial flight is in high demand. The current study simulated a coaxial rotor at a range of flight speeds, 50 knots to 225 knots.

3. Performing an acoustics study to understand and show the effect of interactional aerodynamics on the noise produced by each component.

4. The current work should be extended to include the upper and the lower rotor hubs in the CFD simulations. Rotor hubs contribute substantial amount of drag to the total drag of the helicopter, roughly $20 \%$ to $30 \%$ for a single main 
rotor helicopter [87], which increases for a coaxial helicopter. In addition, the hub way will affect the performance of the rotor blades. The hubs are not present in the CFD simulations, in the current study.

5. Modifying the PRASADUM lift-offset implementation to account for a nonzero phase offset between pilot controls and blade response. The current implementation assumes no phase delay between pilot controls and blade response for a lift-offset coaxial rotor.

6. Including an option for longitudinal lift-offset in the trim equations. Currently, only lateral lift-offset is implemented in the in-house comprehensive analysis.

7. Using the Adaptive Mesh Refinement [79] within Helios to refine and track the vortices as they convent downstream, into the propeller.

8. Improving the main rotor structured blade geometry to have a smoother transition between the elliptical airfoils and the conventional airfoils, as well as a better root and tip mesh quality.

9. Adjusting the time step and implementing a fixed refinement region near the propeller to better capture the flow field near the propeller. The current CFD simulation time step is a quarter degree of the main rotor, which is greater than 1 degree for the propeller.

10. Modifying the geometry of the propeller to have non-linear twist and taper as well as creating a structured O-O grid. The current work utilizes an unstructured grid for the notional propeller, which resulted in slow computations. 


\section{B Coordinate Systems}

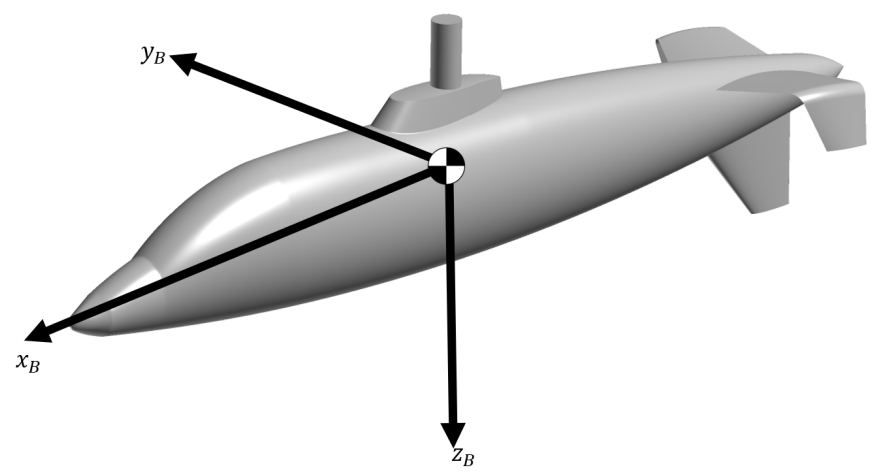

Figure B.1: CSD body-axis frame of reference used for trim procedure.

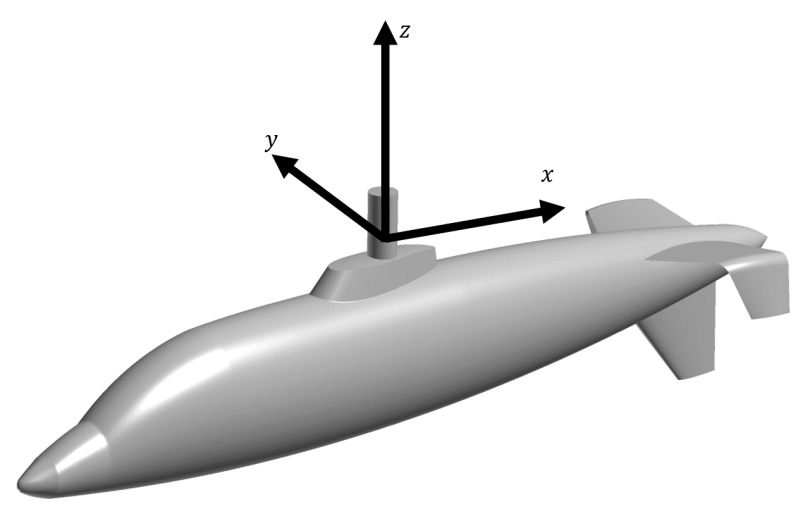

Figure B.2: CFD global axis, utilized for describing CFD airframe airloads. 


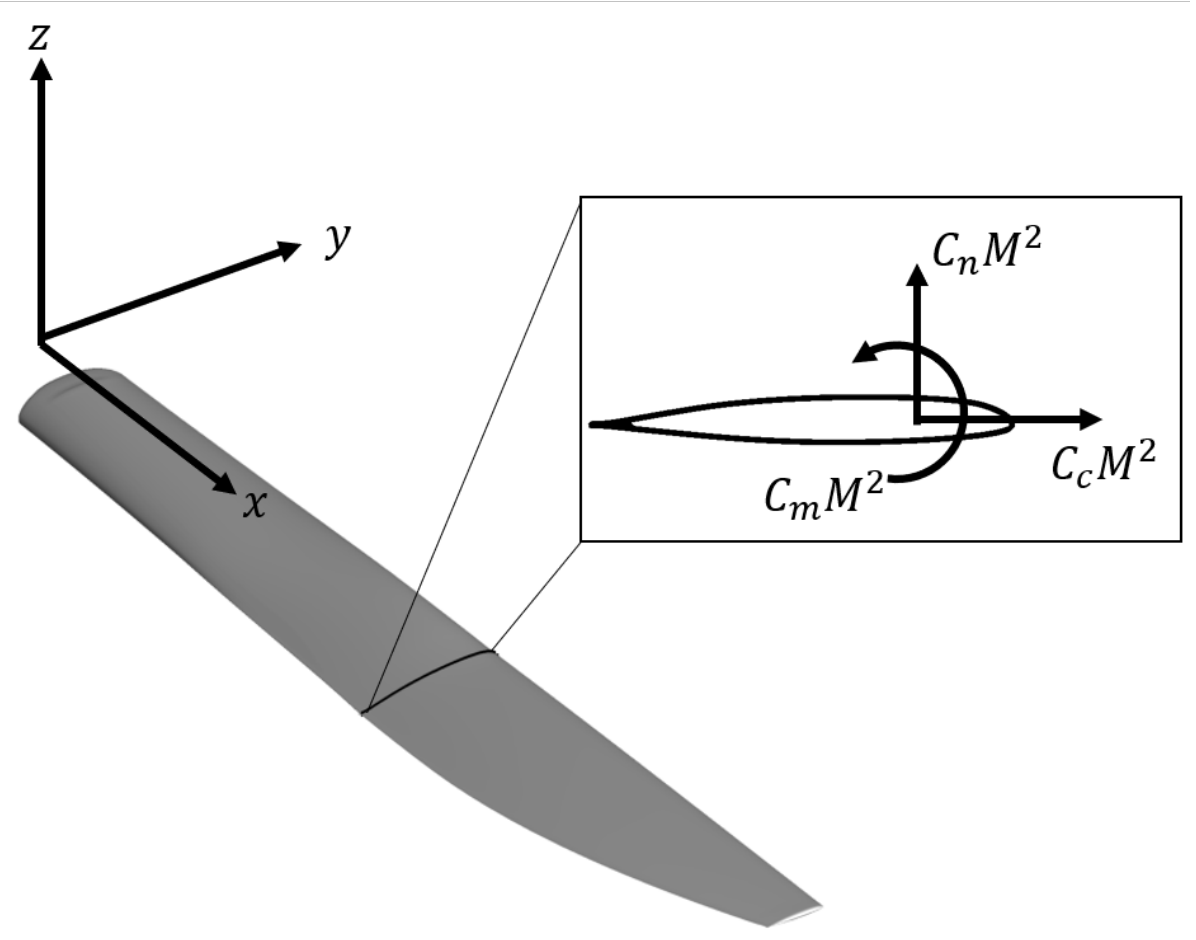

Figure B.3: Reference frame utilized for the non-dimensional deformed airloads, used for delta coupling. 


\section{B Flow Visualization for Results Part I}

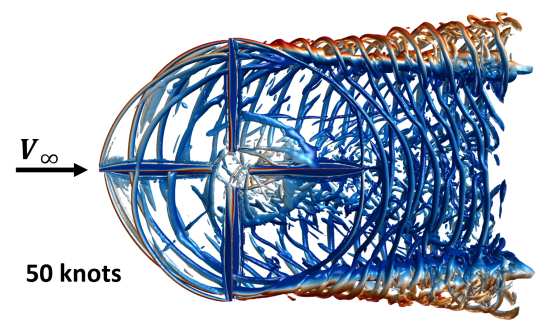

(a) Coaxial rotor System (Top View)

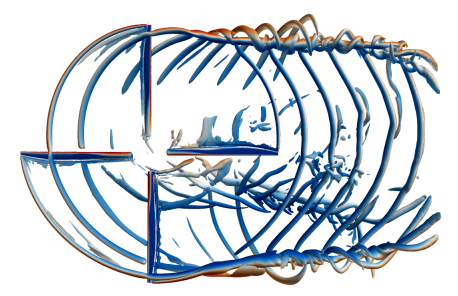

(c) Isolated Lower Rotor (Top View)

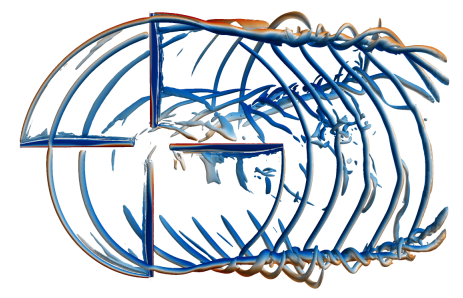

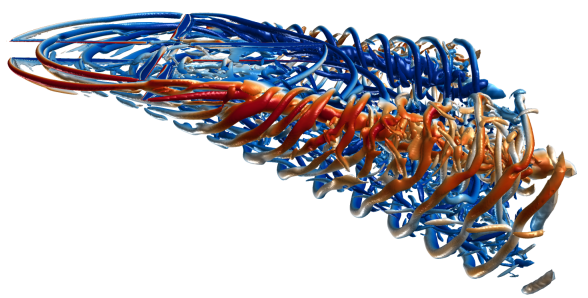

(b) Coaxial rotor System (Starboard View)

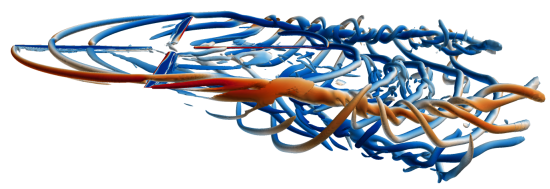

(d) Isolated Lower Rotor (Starboard View)

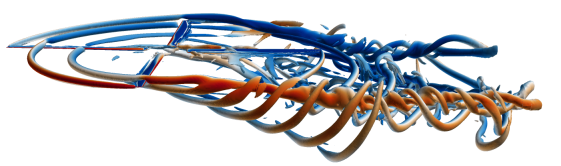

(f) Isolated Upper Rotor (Top View)

Figure B.1: Iso-surfaces of q-criterion, shown for $q=6 \mathrm{e}-4$, colored by the nondimensional vertical velocity, for 50 knot forward flight case. 

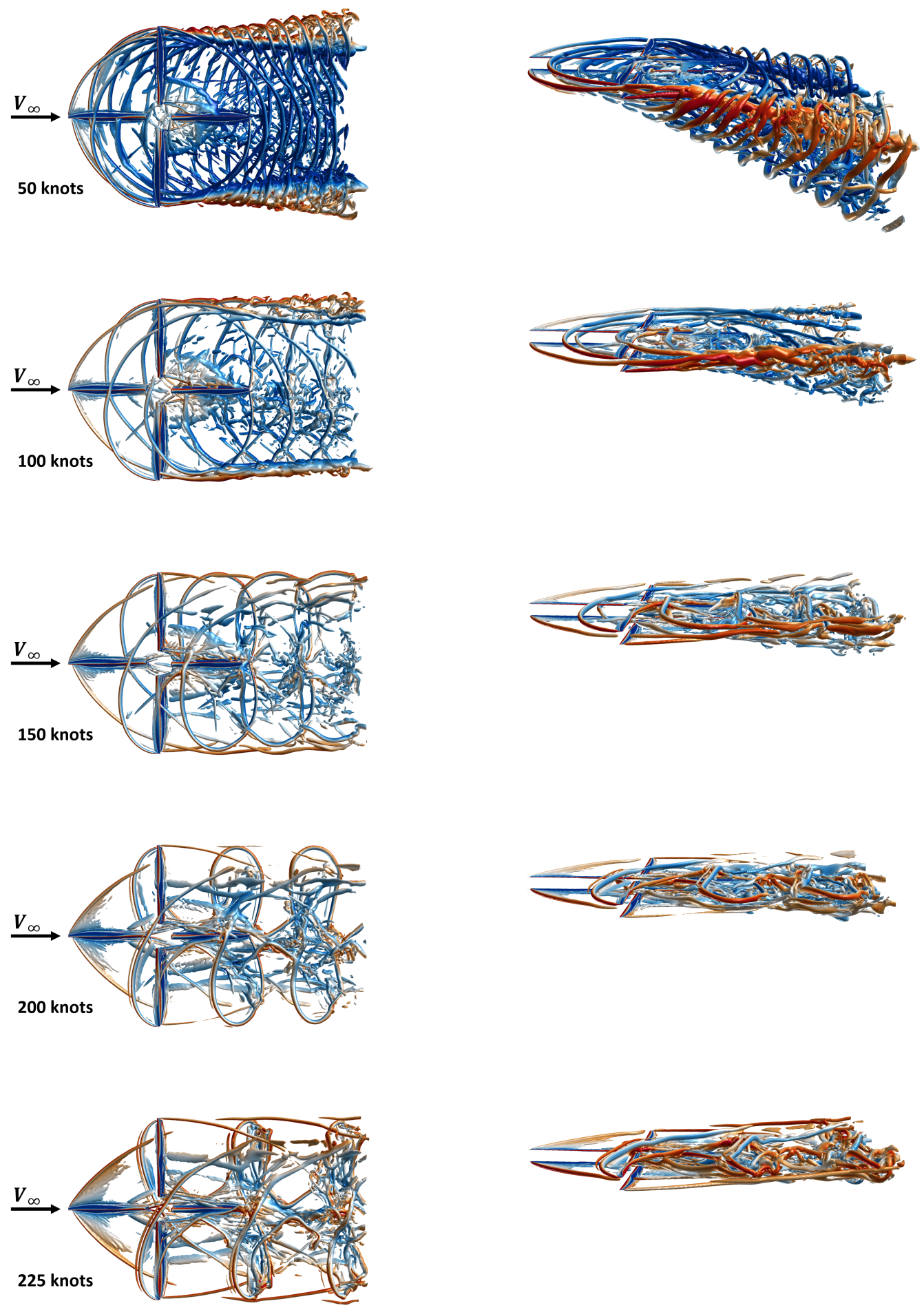

Figure B.2: Iso-surfaces of q-criterion, shown for $q=6 \mathrm{e}-4$, colored by the nondimensional vertical velocity. The right column shows the view of from the top, while the left column shows the view from the starboard side. 


\section{Flow Visualization for Results Part III}

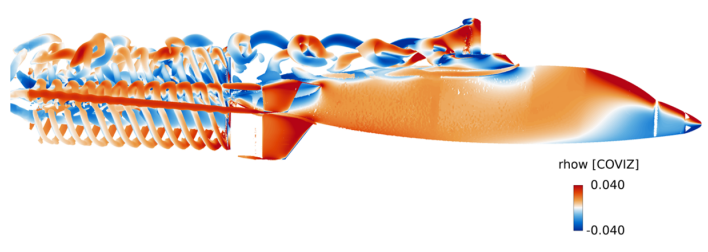

(a) Port View

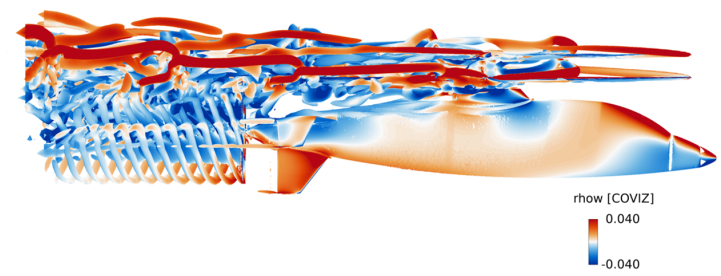

(c) Port View

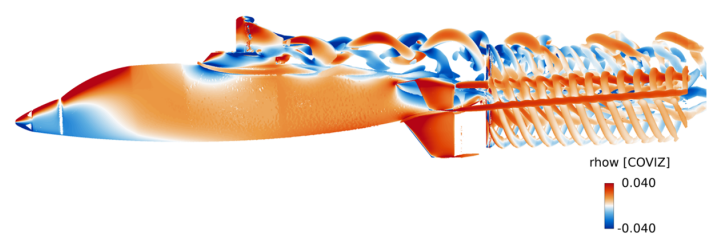

(b) Starboard View

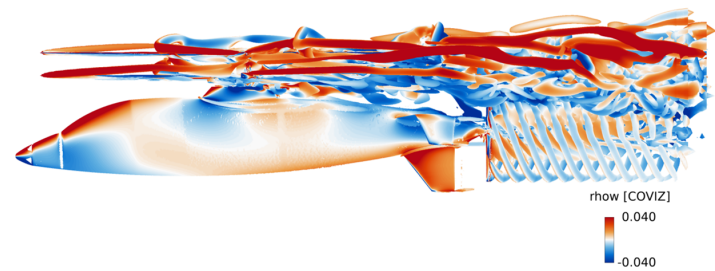

(d) Starboard View

Figure C.1: Iso-surfaces of q-criterion, shown for $q=6 \mathrm{e}-4$, colored by the nondimensional vertical velocity. 

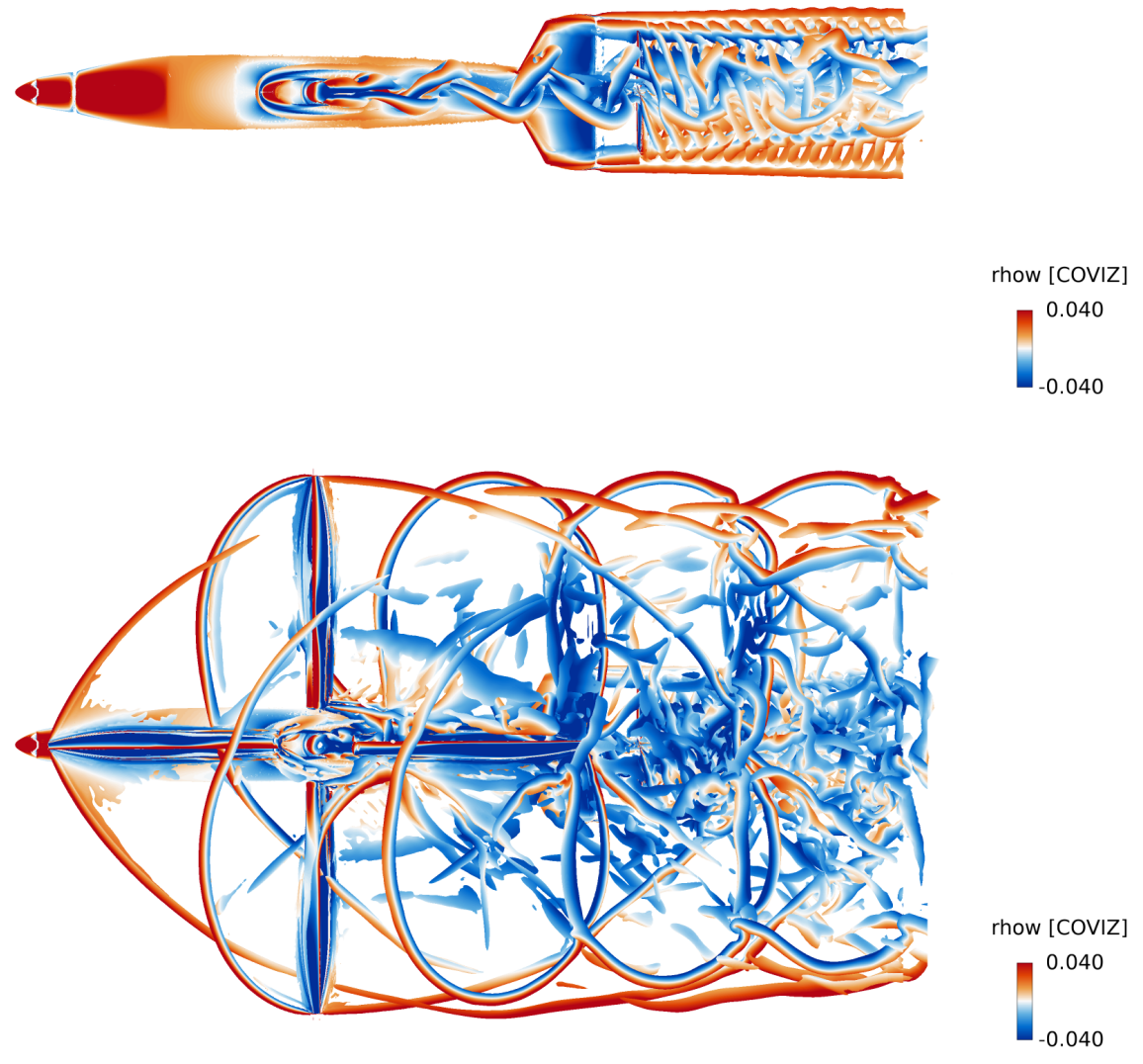

Figure C.2: Top view of the iso-surfaces of q-criterion, shown for $q=6 \mathrm{e}-4$, colored by the non-dimensional vertical velocity. 


\section{Collective Sweep for Isolated Auxiliary Propeller and Airframe- Propeller Combination}

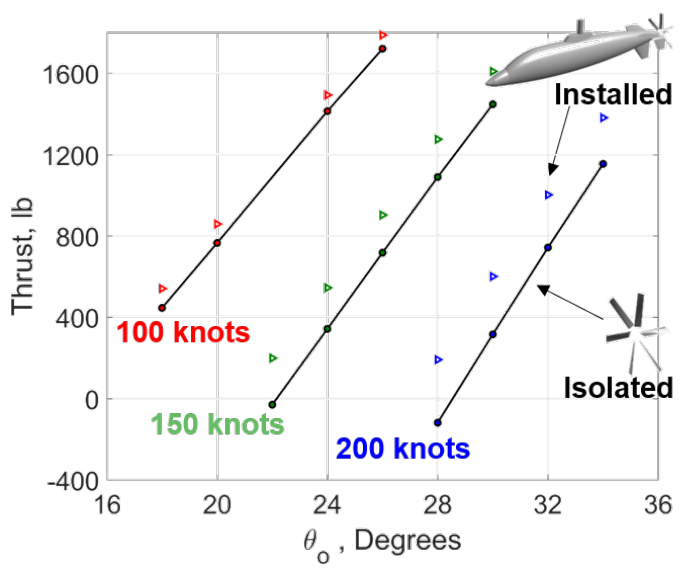

(a) Propeller thrust.

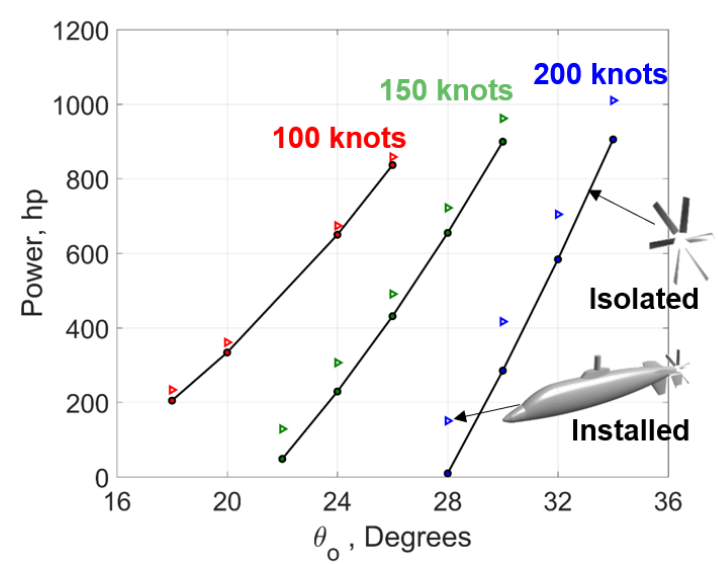

(b) Propeller power.

Figure D.1: Auxiliary propeller collective sweep for isolated and installed propeller at various forward flight speeds. 


\section{Bibliography}

[1] R. Blackwell and T. Millott, "Dynamics design characteristics of the sikorsky x2 technology demonstrator aircraft," in Annual Forum Proceedings-American Helicopter Society, vol. 64, p. 886, AHS,INC, 2008.

[2] R. Biedron and E. Lee-Rausch, "Rotor Airloads Prediction Using Unstructured Meshes and Loose CFD/CSD Coupling," in 26th AIAA Applied Aerodynamics Conference, no. August, (Honolulu, Hawaii), American Institute of Aeronautics and Astronautics, aug 2008.

[3] G. Jacobellis, "Investigation of Blade Loads on a Modern High-Speed LiftOffset Coaxial Helicopter using Coupled Computational Fluid Dynamics / Computational Structural Dynamics," in AHS Aeromechanics Specialists' Meeting, (San Francisco, California), 2018.

[4] "X2 technology demonstrator," May 2010. https://www.flickr.com/photos/ lockheedmartin/29986408154/in/album-72157674412704111/.

[5] A. Bagai, "Aerodynamic design of the x2 technology demonstrator main rotor blade," in 64th Annual Forum of the American Helicopter Society, vol. 64, p. 29, AHS International, 2008.

[6] D. Walsh, S. Weiner, K. Arifian, T. Lawrence, M. Wilson, W. Millott, and R. Blackwell, "High airspeed testing of the sikorsky $\mathrm{x} 2$ technology demonstrator," in American Helicopter Society 67th Annual Forum, Virginia Beach, VA, 2011.

[7] "The worlds fastest military helicopters."

[8] A. J. Ruddell, "Advancing blade concept (abc) development," Journal of the American Helicopter Society, vol. 22, no. 1, pp. 13-23, 1977.

[9] M. Potsdam, H. Yeo, and W. Johnson, "Rotor airloads prediction using loose aerodynamic/structural coupling," Journal of Aircraft, vol. 43, no. 3, pp. 732$742,2006$. 
[10] W. Johnson, "A history of rotorcraft comprehensive analyses," 2013.

[11] H. Saberi, M. Khoshlahjeh, R. A. Ormiston, and M. J. Rutkowski, "Overview of rcas and application to advanced rotorcraft problems," in American Helicopter Society 4th Decennial Specialists Conference on Aeromechanics, San Francisco, $C A, 2004$.

[12] O. A. Bauchau, "Computational schemes for flexible, nonlinear multi-body systems," Multibody System Dynamics, vol. 2, no. 2, pp. 169-225, 1998.

[13] G. Bir, I. Chopra, and K. Nguyen, "Development of umarc (university of maryland advanced rotorcraft code)," 1990.

[14] T. Quackenbush, D. Wachspress, A. Boschitsch, and T. Curbishley, "A comprehensive hierarchical aeromechanics rotorcraft model (charm) for general rotor/surface interaction," CDI Report, pp. 99-03, 1999.

[15] A. Sridharan, Simulation Modeling of Flight Dynamics, Control and Trajectory Optimization of Rotorcraft Towing Submerged Loads. PhD thesis, 2014.

[16] A. Datta, J. Sitaraman, I. Chopra, and J. D. Baeder, "Cfd/csd prediction of rotor vibratory loads in high-speed flight," Journal of aircraft, vol. 43, no. 6, pp. 1698-1709, 2006.

[17] R. D. Harrington, "Full-scale-tunnel investigation of the static-thrust performance of a coaxial helicopter rotor," 1951.

[18] K. McAlister, C. Tung, O. Rand, V. Khromov, and J. Wilson, "Experimental and numerical study of a model coaxial rotor," in American Helicopter Society 62nd Annual Forum, Phoenix, AZ, 2006.

[19] C. Cameron, A. Karpatne, and J. Sirohi, "Performance and vibratory hub loads of a mach-scale coaxial rotor in hover," in 70th American Helicopter Society International Annual Forum 2014, pp. 2647-2655, American Helicopter Society, 2014.

[20] R. C. Dingeldein, Wind-tunnel studies of the performance of multirotor configurations. No. 3236, National Advisory Committee for Aeronautics, 1954.

[21] V. M. Paglino and E. A. Beno, "Full-scale wind tunnel investigation of the advancing blade concept rotor system," tech. rep., UNITED TECHNOLOGIES CORP STRATFORD CT SIKORSKY AIRCRAFT DIV, 1971.

[22] R. Stroub, M. Falarski, J. Mc Cloud, and P. Soderman, "An investigation of a full-scale advancing blade concept rotor system at high advance ratio," 1971.

[23] F. F. Felker III, "Performance and loads data from a wind tunnel test of a fullscale, coaxial, hingeless rotor helicopter.," tech. rep., NATIONAL AERONUATICS AND SPACE ADMINISTRATION MOFFETT FIELD CA AMES RESEARCH , 1981. 
[24] C. Cameron and J. Sirohi, "Performance and loads of a model coaxial rotor part i: Wind tunnel testing," in American Helicopter Society 72nd Annual Forum, pp. 1-15, American Helicopter Soc. Fairfax, VA, 2016.

[25] P. Lorber, P. Bowles, E. Fox, and R. Park, "Wind Tunnel Testing for the SB $>1$ Defiant Joint Multi-Role Technology Demonstrator," in AHS International 73rd Annual Forum, (Fort Worth, Texas), pp. 1-18, 2017.

[26] P. F. Lorber, B.-y. Min, and J. Zhao, "COMPARISON OF ROTOR FUSELAGE FLOW FIELDS AND UNSTEADY TAIL INTERACTIONS BETWEEN TWO CFD CODES AND EXPERIMENT," in Vertical Flight Society's 75th Annual Forum \&3 Technology Display, (Philadelphia), 2019.

[27] D. N. Arents, "An assessment of the hover performance of the xh-59a advancing blade concept demonstration helicopter," tech. rep., ARMY AIR MOBILITY RESEARCH AND DEVELOPMENT LAB FORT EUSTIS VA EUSTIS DIRECTORATE, 1977.

[28] A. Akimov, V. Butov, B. Bourtsev, and S. Selemenev, "Flight investigation of coaxial rotor tip vortex structure," in ANNUAL FORUM PROCEEDINGSAMERICAN HELICOPTER SOCIETY, vol. 50, pp. 1431-1431, AMERICAN HELICOPTER SOCIETY, 1994.

[29] A. Datta, M. Nixon, and I. Chopra, "Review of rotor loads prediction with the emergence of rotorcraft cfd," Journal of the American Helicopter Society, vol. 52, no. 4, pp. 287-317, 2007.

[30] A. Datta and I. Chopra, "Prediction of the uh-60a main rotor structural loads using computational fluid dynamics/comprehensive analysis coupling," Journal of the American Helicopter Society, vol. 53, no. 4, pp. 351-365, 2008.

[31] M. J. Smith, J. W. Lim, B. G. van der Wall, J. D. Baeder, R. T. Biedron, D. D. Boyd, B. Jayaraman, S. N. Jung, and B.-Y. Min, "The HART II international workshop: an assessment of the state of the art in CFD/CSD prediction," CEAS Aeronautical Journal, vol. 4, pp. 345-372, dec 2013.

[32] D. D. Boyd Jr, "HART-II Acoustic Predictions using a Coupled CFD / CSD Method," in American Helicopter Society 65th Annual Forum, (Grapevine), pp. 1-19, 2009.

[33] M. Amiraux, NUMERICAL SIMULATION AND VALIDATION OF HELICOPTER BLADE-VORTEX INTERACTION USING COUPLED CFD / CSD AND THREE LEVELS OF AERODYNAMIC MODELING. Doctor of philosophy, University of Maryland, 2014.

[34] B. Roget, J. Sitaraman, A. Wissink, H. Saberi, and W. Chen, "Maneuvering Rotorcraft Simulations Using HPCMP CREATE-AV Helios," in 54th AIAA Aerospace Sciences Meeting, no. January, (San Diego), 2016. 
[35] M. Embacher, M. Keßler, M. Dietz, and E. Krämer, "Coupled CFD-Simulation of a Helicopter in Free-Flight Trim," in American Helicopter Society 66th Annual Forum, (Phoenix,AZ), 2010.

[36] B. Roget, M. Field, and R. Blumenstein, "Advanced Rotorcraft Aeromechanics Simulations using HPCMP CREATE TM -AV Helios," in Vertical Flight Society 75th Annual Forum \& Technology Display, (Philadelphia), 2019.

[37] B. J. Passe, A. Sridharan, and J. D. Baeder, "Computational investigation of coaxial rotor interactional aerodynamics in steady forward flight," in 33rd AIAA Applied Aerodynamics Conference, p. 2883, 2015.

[38] V. Klimchenko, A. Sridharan, and J. D. Baeder, "Cfd/csd study of the aerodynamic interactions of a coaxial rotor in high-speed forward flight," in 35th AIAA Applied Aerodynamics Conference, p. 4454, 2017.

[39] Z. Jia and S. Lee, "Impulsive loading noise of a lift-offset coaxial rotor in highspeed forward flight," AIAA Journal, pp. 1-15, 2019.

[40] M. Bhagwat and E. T. Meadowcroft, "CFD / CSD Coupled Trim Solution for the Dual-Rotor CH-47 Helicopter Including Fuselage Modeling," in American Helicopter Society Specialist's Conference on Aeromechanics, (San Francisco, CA), pp. 1-8, 2008.

[41] J. Zhao, M. Brigley, R. Modarres, and W. A. Welsh, "S-97 raider rotor vibratory loads analysis using cfd-csd," in AIAA Scitech 2019 Forum, p. 0860, 2019.

[42] P. O. Bowles, B. E. Wake, C. Matalanis, M. Battisti, B.-y. Min, P. F. Lorber, and N. Tuozzo, "Full-Configuration CFD Analysis of the S-97 RAIDER," in VFS 75th Annual Forum \& Technology Display, no. May, (Philadelphia), pp. 1$12,2019$.

[43] P. Anusonti-Inthra, "The Effects of the Fuselage on a Coaxial Rotorcraft Performance, Aerodynamics, and Structural Dynamics," in AIAA Scitech 2019 Forum, no. January, (San Diego, California), pp. 1-15, American Institute of Aeronautics and Astronautics, jan 2019.

[44] B. Passe, A. Sridharan, and J. Baeder, "Identification of Rotor-Fuselage Aerodynamic Interactions in a Compound Coaxial Helicopter using CFD-CSD Coupling," in American Helicopter Society Aeromechanics Design for Vertical Lift, (San Francisco, CA), 2016.

[45] B. Passe, "Simulation of coaxial rotor interactional aerodynamics using coupled cfd-csd," Master's thesis, 2015.

[46] W. Johnson, "Lift-Offset Compound Design Background, X2TD, JMR ME1A Status and Plans," (Ames Research Center, Moffett Field, California), 2011. 
[47] X. Wang, Y. S. Jung, J. Baeder, and I. Chopra, "CFD Pressure/Airload Correlation with Experimental Data on a Slowed Mach-Scaled Rotor at High Advance Ratios," in 74th Annual Forum of the American Helicopter Society, (Phoenix, Arizona), 2018.

[48] R. Celi, "Lecture notes in ENAE635 - Helicopter Stability and Control ."

[49] C. He and D. A. Peters, "Comparison of Measured Induced Velocities with Results from a Closed-form Finite State Wake Model in Forward Flight," in $45^{\text {th }}$ Annual National Forum of the American Helicopter Society, AHS, May 1989.

[50] A. Sridharan, "PRASADUM Theory Manual," 2017.

[51] R. Celi, "UMD HeliUM Program Documentation,” 2008.

[52] J. J. Moré, B. S. Garbow, and K. E. Hillstrom, "User guide for minpack-1," tech. rep., CM-P00068642, 1980.

[53] S. Sidle, A. Sridharan, and I. Chopra, "Coupled vibration prediction of rotorairframe-drivetrain-engine dynamics," in AHS 74th Annual Forum, Phoenix, Arizona, 2018.

[54] D. P. N. Jude, Advancing the Multi-Solver Paradigm for Overset CFD toward Heterogeneous Architectures. PhD thesis, University of Maryland, College Park, 2019.

[55] S. Sidle, A. Sridharan, I. Chopra, M. Feshler, and P. Kull, "Investigation of engine-airframe vibration due to main rotor hub loads using a substructuring framework," Journal of the American Helicopter Society, vol. 64, no. 4, pp. 1$16,2019$.

[56] A. Bagai and J. Leishman, "The maryland free-wake analysis-theory, implementation and users manual," University of Maryland, Department of Aerospace Engineering, Technical Report Prepared for NASA Langley Research Center, Aeroacousitics Branch, Fluid Mechanics and Acoustics Division, Contract, no. 015-2685, 1995.

[57] T. H. Pulliam and J. L. Steger, "Implicit finite-difference simulations of threedimensional compressible flow," AIAA Journal, vol. 18, no. 2, pp. 159-167, 1980 .

[58] J. D. Anderson and J. Wendt, Computational fluid dynamics, vol. 206. Springer, 1995.

[59] R. E. Sonntag, Fundamentals of Thermodynamics. John Wiley and Sons, 2003.

[60] S. B. Pope, "Turbulent flows," 2001. 
[61] P. K. Kundu and I. M. Cohen, "Fluid mechanics," Fluid Mechanics: Fourth Edition. Edited by Pijush K. Kundu and Ira M. Cohen with contributions by PS Ayyaswamy and HH Hu. ISBN 978-0-12-373735-9. Published by Academic Press, Elsevier, Inc., London, England, 2008., 2008.

[62] F. G. Schmitt, "About boussinesq's turbulent viscosity hypothesis: historical remarks and a direct evaluation of its validity," Comptes Rendus Mécanique, vol. 335, no. 9-10, pp. 617-627, 2007.

[63] P. Spalart and S. Allmaras, "A one-equation turbulence model for aerodynamic flows," in 30th aerospace sciences meeting and exhibit, p. 439, 1992.

[64] A. M. Wissink, W. J. Staruk, S. A. Tran, B. Roget, V. K. Lakshminarayan, J. Sitaraman, and B. Jayaraman, "Overview of new capabilities in helios version 9.0," in AIAA Scitech 2019 Forum, p. 0839, 2019.

[65] V. Sankaran, A. Wissink, A. Datta, J. Sitaraman, M. Potsdam, B. Jayaraman, A. Katz, S. Kamkar, B. Roget, D. Mavriplis, et al., "Overview of the helios version 2.0 computational platform for rotorcraft simulations," in 49th AIAA Aerospace Sciences Meeting including the New Horizons Forum and Aerospace Exposition, p. 1105, 2011.

[66] A. Wissink, B. Jayaraman, A. Datta, J. Sitaraman, M. Potsdam, S. Kamkar, D. Mavriplis, Z. Yang, R. Jain, J. Lim, et al., "Capability enhancements in version 3 of the helios high-fidelity rotorcraft simulation code," in 50th AIAA Aerospace Sciences Meeting including the New Horizons Forum and Aerospace Exposition, p. 713, 2012.

[67] A. Wissink, J. Sitaraman, B. Jayaraman, B. Roget, V. Lakshminarayan, M. Potsdam, R. Jain, J. Leffell, J. R. Forsythe, and A. Bauer, "Recent advancements in the helios rotorcraft simulation code," in 54th AIAA Aerospace Sciences Meeting, pp. 2016-0563, 2016.

[68] J. Sitaraman, M. Floros, A. Wissink, and M. Potsdam, "Parallel domain connectivity algorithm for unsteady flow computations using overlapping and adaptive grids," Journal of Computational Physics, vol. 229, no. 12, pp. 4703-4723, 2010 .

[69] B. Roget, J. Sitaraman, A. Wissink, H. Saberi, and W. Chen, "Maneuvering Rotorcraft Simulations Using HPCMP CREATE T M -AV Helios," in 54th AIAA Aerospace Sciences Meeting, no. January, (San Diego, California), 2016.

[70] R. H. Nichols and P. G. Buning, "Users manual for overflow 2.2," University of Alabama and NASA Langley Research Center, 2019.

[71] T. Pulliam, "High order accurate finite-difference methods: as seen in overflow," in 20th AIAA Computational Fluid Dynamics Conference, p. 3851, 2011. 
[72] A. M. Wissink, S. Kamkar, T. H. Pulliam, J. Sitaraman, and V. Sankaran, "Cartesian Adaptive Mesh Refinement for Rotorcraft Wake Resolution," in 28th AIA A Applied Aerodynamics Conference, no. July, (Chicago, Illinois), pp. 1-18, 2010.

[73] R. K. Jain and M. A. Potsdam, "Hover Predictions on the Sikorsky S-76 Rotor using Helios," in 52nd Aerospace Sciences Meeting, no. January, (National Harbor, Maryland), pp. 1-22, 2014.

[74] P. L. Roe, "Approximate riemann solvers, parameter vectors, and difference schemes," Journal of computational physics, vol. 43, no. 2, pp. 357-372, 1981.

[75] J. Blazek, Computational fluid dynamics: principles and applications. Butterworth-Heinemann, 2015.

[76] H. Lomax, T. H. Pulliam, and D. W. Zingg, Fundamentals of computational fluid dynamics. Springer Science \& Business Media, 2013.

[77] R. T. Biedron, J.-R. Carlson, J. M. Derlaga, P. A. Gnoffo, D. P. Hammond, W. T. Jones, B. Kleb, E. M. Lee-Rausch, E. J. Nielsen, M. A. Park, et al., "Fun3d manual: 13.5," 2019.

[78] E. Mestreau, R. Aubry, S. Dey, and M. Richardson, "Hpcmp create capstone design and architecture 'i\&' feature modeling improvements," in AIAA Scitech 2019 Forum, p. 1716, 2019.

[79] A. Wissink, S. Kamkar, T. Pulliam, J. Sitaraman, and V. Sankaran, "Cartesian adaptive mesh refinement for rotorcraft wake resolution," in 28th AIAA Applied Aerodynamics Conference, p. 4554, 2010.

[80] W. G. Bousman, Putting the aero back into aeroelasticity. National aeronautics and space administration moffett field ca ames research , 2000.

[81] O. Bauchau and J. Ahmad, "Advanced cfd and csd methods for multidisciplinary applications in rotorcraft problems," in 6th Symposium on Multidisciplinary Analysis and Optimization, p. 4151, 1996.

[82] E. T. Meadowcroft and R. Jain, "Improvements to Tandem-Rotor H-47 Helicopter Coupled CFD-CSD Full Aircraft Model," in AHS 72nd Annual Forum, (West Palm Beach, Florida,), 2016.

[83] T. R. Norman, P. Shinoda, R. L. Peterson, and A. Datta, "Full-scale wind tunnel test of the uh-60a airloads rotor," tech. rep., ARMY RESEARCH DEVELOPMENT AND ENGINEERING COMMAND MOFFETT FIELD CA AVIATION , 2011.

[84] T. Meadowcroft, "Advanced aeromechanics prediction methods for rotorcraft flight vehicles," 2017. 
[85] V. Klimchenko and J. D. Baeder, "Cfd/csd study of interactional aerodynamics of a coaxial compound helicopter in high-speed forward flight," in AIAA Scitech 2020 Forum, p. 0304, 2020.

[86] B.-Y. Min, B. Moffitt, P. O. Bowles, B. E. Wake, and P. F. Lorber, "Analysis and Experimental Testing of S-97 RAIDER Propeller," in AHS Specialists' Conference on Aeromechanics Design for Transformative Vertical Flight, (San Francisco), AHS International, 2018.

[87] D. R. Graham, D. Y. Sung, L. A. Young, A. W. Louie, and R. H. Stroub, "Helicopter Hub Fairing and Pylon Interference Drag," tech. rep., NASA, Moffett Field, California, 1989. 